\title{
When Staff \\ Handle Staph
}

\section{User-Driven Versus Expert-Driven Communication Of Infection Control Guidelines}

Fenne Verhoeven 
Thesis, University of Twente, 2009.

ISBN: 978-90-365-2868-9

(C) 2009 Fenne Verhoeven

Cover and book design by Mark Lindhout (www.langdradig.nl)

Cover photography by Frank Muller (www.zorginbeeld.nl)

Printed by Gildeprint Drukkerijen BV, Enschede, the Netherlands

The studies presented in this thesis were financially supported by the EUREGIO MRSAnet Twente/Münsterland under the European Regional Development Fund (ERDF), which is funded by the European Union within the Community Initiative INTERREGIIIA (2-EUR-V-1=96), as well as by the Ministry of Economics in the German state of Nordrhein-Westfalen. 


\section{WHEN STAFF HANDLE STAPH \\ USER-DRIVEN VERSUS EXPERT-DRIVEN COMMUNICATION OF INFECTION CONTROL GUIDELINES}

PROEFSCHRIFT

ter verkrijging van

de graad van doctor aan de Universiteit Twente,

op gezag van de rector magnificus,

prof. dr. H. Brinksma,

volgens besluit van het College voor Promoties

in het openbaar te verdedigen

op vrijdag 2 oktober 2009 om 15.00 uur

door

Fenne Verhoeven

geboren op 21 september 1981

te Aarle-Rixtel 
Dit proefschrift is goedgekeurd door de promotor, prof. dr. M.F. Steehouder, en de assistent-promotor, dr.J.E.W.C. van Gemert-Pijnen 
Samenstelling promotiecommissie:

Promotor: $\quad$ Prof. dr. M.F. Steehouder, Universiteit Twente

Assistent-promotor: Dr.J.E.W.C. van Gemert-Pijnen, Universiteit Twente

Leden: $\quad$ Prof. dr. G. Eysenbach, University of Toronto

Dr. M.G.R. Hendrix, Laboratorium Microbiologie

Twente Achterhoek

Prof. dr. F.M.G. de Jong, Universiteit Twente

Prof. dr. E.R. Seydel, Universiteit Twente

Prof. dr. H. Vondeling, Universiteit Twente

Prof. dr. W. Witte, Robert Koch Institut 


\section{Contents}

1 Introduction

2 Communication of infection control guidelines from a global health perspective: a conceptual framework

3 The development of a web-based information tool for cross-border prevention and control of Methicillin Resistant Staphylococcus aureus

4 From expert-driven to user-oriented communication of infection control guidelines

5 How nurses seek and evaluate clinical practice guidelines on the Internet

6 Factors affecting health care workers' adoption of a website with infection control guidelines

7 Identifying intervention strategies for guideline adherence based on health care workers' perceived barriers and facilitators

8 Conclusions and discussion

References

Samenvatting (Summary in Dutch)

Dankwoord (Acknowledgements in Dutch) 
Chapter 1

Introduction 



\section{Introduction}

Does a web-based presentation of infection control guidelines improve their usability over a paper-based approach? This thesis is focused on this question. This first chapter provides the research background and motivates the need for a user-driven approach of the design process of such a website. First, the research context is presented, followed by the model that underpins the design process. Then, the research questions and methodology are described. The chapter ends with an overview of the thesis.

\subsection{Background}

\subsubsection{Research context: EUREGIO MRSA-net Twente/ Münsterland}

“Primum non nocere- First, do no harm." Hippocrates (ca. $460-$ ca. 377 B.C.)

This phrase is a fundamental principle for all health care workers (HCWs). However, despite these workers' extraordinary effort and best intentions, thousands of patients are inadvertently harmed in hospitals worldwide, every day (De Vries et al., 2008). Health care-associated infections (HAIs), which patients acquire while receiving treatment for other diseases, are among the top ten leading causes of death around the world (Centers for Disease Control and Prevention, Atlanta, 2009). More specifically, HAls cause approximately 99.000 attributable deaths in the United States and 50.000 in the European Union each year (Health First Europe, 2009; Klevens et al., 2007).

One of the organisms that most commonly contribute to the rapid spread of HAls, is Methicillin Resistant Staphylococcus aureus (MRSA) (Friedrich et al., 2008). This is a variant of the common bacterium Staphylococcus aureus. This bacterium has evolved an ability to survive treatment with several classes of antibiotics, including methicillin. Patients with wounds, invasive devices, and weakened immune systems can become infected with MRSA with serious consequences such as pneumonia and sepsis (blood poisoning) and in the worst case death (Centers for Disease Control and Prevention, Atlanta, 2009). Carried by healthy people, MRSA is usually harmless. However, a newer form of MRSA infection, known as Community-Acquired MRSA has appeared with increasing frequency. Community-Acquired MRSA is now epidemic within certain community populations among persons without any established risk factors. Given that MRSA also appears to be ever more prevailing among cattle and in raw meat since 2005 and 2007 respectively, MRSA is becoming a major public health issue, affecting thousands of otherwise healthy people (Van Loo et al., 2007; Voss et al., 2005). 
Next to an increased mortality and morbidity rate, HAls caused by MRSA and other micro-organisms lead to extreme costs due to increased staffing needs, prolonged hospital stays, antibiotic treatments, etc. (Gould, 2006). Also, depressive and anxious symptoms ubiquitously prevail among MRSA-infected patients (Tarzi et al., 2001). The occurrence of HAls is detrimental both to patients' and HCWs' safety and, consequently, to quality of care (Pittet \& Donaldson, 2006).

Increased international trade in health services can be an important cause of the rapid spread of MRSA (Bettcher \& Lee, 2002; Pittet et al., 2005). This particularly holds for European borders, where the cross-border movement of HCWs and patients to supply and access medical services abroad heightens the threat of cross-border MRSA transmission, particularly when patients and HCWs are transferred from a middleor high-endemic country, such as Germany, to a low-endemic country, such as the Netherlands, with an MRSA prevalence of $23 \%$ and $5 \%$, respectively (Friedrich et al., 2008; Pittet et al., 2005). These different MRSA rates stem from differences in the resources provided and the priority given to infection control programs by national authorities (Humphreys, 2006). The Dutch MRSA policy has a so-called "search and destroy" character, comprised of the rigid isolation and screening of high-risk patient groups, the screening of low-risk groups, the strict isolation of carriers, and the treatment of people carrying MRSA. Furthermore, this "search and destroy" policy operates in an environment where MRSA is not endemic, with different indications for screening, better facilities for patient isolation (Harbarth \& Pittet, 2005; Humphreys, 2006), and a long-standing tradition in parsimonious antibiotic use in contrast to Germany, where the opposite is the case. Also, the national MRSA guidelines issued by the Dutch Working party on Infection Prevention (2005a) are considered professional standards and are used as such by the Dutch public health inspector. This undoubtedly contributes to the adherence to the guidelines by nearly all Dutch health institutions (Harbarth \& Pittet, 2005).

In order to ensure patient safety and to equalize quality of care in cross-border health care settings, the prevention of HAls needs to be structurally addressed by synergic efforts of the health care actors representing the complete chain of care, on both sides of the border. An example of such an effort is EUREGIO MRSA-net Twente/Münsterland, in which local health care providers from both Germany and the Netherlands exchange knowledge and technology in order to reduce MRSA to acceptable levels both inside and outside the hospital (Friedrich et al., 2006, 2007). EUREGIO MRSA-net Twente/Münsterland is funded by the European Union. In Germany, the project is coordinated by the Institute for Hygiene at the Münster University Hospital and the State Institute for Health and Work in North Rhine-Westphalia. In the Netherlands, the project is managed by the public health laboratory TwenteAchterhoek and the University of Twente in Enschede. Altogether, 40 hospitals in a region covering 8000 square kilometres and comprised of 2.7 million inhabitants ( 950000 inhabitants in the Netherlands area) have participated in the project.

Since its launch in 2005 , this project has founded a cross-border network of the major health care providers (e.g., hospitals, labs, public health services, and general practitioners) that have jointly developed an effective infection control strategy, 
including surveillance, early detection, and better education about HAls for both HCWs and patients. The educational part of the efforts is facilitated by a website that promotes HCWs' adherence to clinical practice guidelines for the prevention and control of MRSA (Friedrich et al., 2008). This thesis focuses on the design process of this particular website.

\subsubsection{Problems with existing infection control guidelines}

Clinical practice guidelines are "systematically developed statements to assist practitioner and patient decisions about appropriate health care for specific clinical circumstances" (Lohr \& Field, 1992). Their purpose is "to make explicit recommendations with a definite intent to influence what clinicians do" (Hayward et al., 1995). Clinical practice guidelines are formulated from the perspective of evidence-based medicine, which is the conscientious, explicit, and judicious use of current best evidence in making decisions about the care of individual patients (Sackett et al., 1996).

Clinical practice guidelines aimed at the prevention and control of MRSA include hand hygiene, gloving, protection for the eye, mouth, and nose, gowning, surveillance cultures, patient isolation, and the appropriate handling of patient care equipment, instruments/devices, and laundry (Muto et al., 2003). The implementation of infection control guidelines has been described as the most promising factor for tackling HAls (World Alliance for Patient Safety, 2005). Therefore, it is of vital importance that HCWs adhere to these guidelines (Miller \& Kearny, 2004).

Although most HCWs are aware of the rationale for infection control guidelines, their adherence is generally poor (Gershon et al., 2000; Pittet et al., 2005). Research suggests that adherence is complex, multi-faceted, and influenced by two groups of factors (Francke et al., 2008):

\section{Quality of documentation}

The low adherence rates seem partly due to the insufficient quality of infection control guidelines as a communication means. Van Gemert-Pijnen (2003) demonstrated that the majority of HCWs encounter serious problems with accessing, understanding, applying, and accepting the infection control guidelines. According to Van Gemert-Pijnen, the problem with the format of infection control guidelines as a communication means has four related causes:

1) The current infection control guidelines have two hard to reconcile functions. They serve as documentation of the health and safety policy of the healthcare institution ("regulation function") and as a communication means for individual HCWs ("communication function") (cf. also Elling, 1991). Since the first function prevails, the second function often plays a subordinate part;

2) The content of the guidelines is predominantly defined by infection control professionals, resulting in the expert-driven character of the guidelines. In the 
design process, higher priority is given to a consensus on content-related issues among experts than to HCWs' practical information needs. This can make the document difficult for individual HCWs to use as a resource and to identify procedures for daily work practice (The AGREE Collaboration, 2003; Van GemertPijnen et al., 2005). This results in hard to read guidelines that are insufficiently targeted to HCWs' norms and values, and eventually provoke reluctance and disregard among HCWs.

3) Infection control guidelines are expert-driven rather than user-driven: HCWs' tacit knowledge, which is context-dependent and made up of the practical and experiential wisdom of individual HCWs, is rarely or never externalized in guidelines (cf. also Williams \& Dickinson, 2008). This leads to a discrepancy between HCWs' perception of their environment and the guidelines;

4) Infection control guidelines are traditionally communicated via a paper-based format. This may imply, in daily work practice, that the documents are hard to locate at the time of need.

\section{Context of use}

Next to documentation quality, the context of use includes several factors that contribute to HCWs' poor adherence to infection control guidelines. These factors can be divided into three groups:

1) Personal factors, e.g., many HCWs perceive infection control guidelines as a stifling innovation that erodes trust;

2) Work-related factors, such as workload and unavailability of personal protective equipment;

3) Organizational factors, such as the lack of management or peer support, but also inadequate training and feedback.

This diagnosis suggests that strategies aiming to enhance HCWs' adherence to infection control guidelines will only be effective when both document quality and context of use are taken into consideration (Francke et al., 2008; Kukafka et al., 2003). An excellent way of combining these considerations might be the development of a user-driven website for the communication of infection control guidelines. 


\subsubsection{From expert-driven to user-driven communication of infection control guidelines}

\section{Quality of documentation}

As explained in section 1.1.2., expert-driven guidelines can be characterized by a strong focus on scientific validation, regulation, and legislation (The AGREE Collaboration, 2003). This is not surprising, since the guidelines' main function is to document safety regulations. However, since content-related issues dominate the infection control guidelines, the guidelines are less appropriate for individual HCWs to search for context-specific procedures. In order to do so, the existing evidence-based guidelines should be presented in a user-driven manner. It should be emphasized that the content of the guidelines should never be subject to change when developing a more user-driven means of communicating infection control guidelines. The evidencebased content of the guidelines should always be considered, irrespective of the way in which the guidelines are communicated (Sackett et al., 1996).

User-driven communication of infection control guidelines implies that the guidelines are rephrased in a more instructional style, words are used with which HCWs are familiar rather than jargon, multimedia are used to visualize the guidelines, etc. Nevertheless, the evidence-based content should be always maintained. In user-driven communication of infection control guidelines, HCWs (users) provide input regarding the communication style of the guidelines, but medical microbiologists (experts) should always verify the clinical content of the guidelines.

A promising solution for the user-driven communication of infection control guidelines is to communicate guidelines via a website. A website allows the integration of multiple perspectives towards information by providing a hyperlink structure, which makes it possible to reconcile the two functions ("regulation" and "communication") and the two perspectives (expert and user) (Fervers et al., 2005). Moreover, a website with infection control guidelines has the potential to be incorporated into the workflow of clinical care due to its ability to provide concise, relevant clinical information at the location and time of need, in contrast to traditional paper-based guidelines. It is therefore not surprising that earlier research has reported better adherence of HCWs to electronic guidelines than to traditional paper-based guidelines (Thomas et al., 1999). Other reported benefits of web-based guideline communication are a decrease of variation in practices between clinicians, and improved patient outcomes (Garg et al., 2005; Grimshaw \& Russell, 1993).

The design of a website to communicate infection control guidelines allows the direct involvement of HCWs, which can lead to a more user-driven approach and a better fit with HCWs' norms and values. HCWs are invited to make their tacit knowledge concerning infection control explicit, are inspired to make their own decisions about directions and strategies for improvement, and are led to action. Involving HCWs in the development process is important in creating a sense of ownership and fostering the guidelines' applicability (Elling, 1991; Van Gemert-Pijnen, 2003). Again, it should be pointed out that HCWs can only be consulted to elicit requirements regarding the 
guidelines' presentation and communication style. The evidence-based content of the guidelines should always be warranted and verified by a clinical expert, such as a medical microbiologist.

In order to compare the usability of a web-based format with the traditional paperbased guidelines, it is essential to define outcome measures of usability. The main standard that provides guidance on usability is ISO 9241, which describes usability as "the extent to which a product can be used by specified users to achieve specified goals with effectiveness, efficiency, and satisfaction in a specified context of use" (International Organization for Standardization, 1998). Frøkjær et al. (2000) relate the ISO definition of usability to more specific outcome measures for each of the three aspects of usability: effectiveness, efficiency, and satisfaction. Applied to the context of this thesis, this implies that a website with infection control guidelines should enable HCWs to:

- successfully retrieve complete, comprehensible, and accurate guidelines (effectiveness);

- retrieve guidelines with less time and effort compared to the case of paper-based guidelines (efficiency);

- be comfortable with and hold positive attitudes towards it (satisfaction).

\section{Context of use}

Despite the possible contribution of a web-based, user-driven format to the quality of guideline documentation, numerous obstacles to the adoption of a website with infection control guidelines in daily work practice remain (Jeannot et al., 2003). In this thesis, adoption refers to "the process by which individuals and groups decide to use a website for the retrieval of infection control guidelines." Adoption is a prerequisite for the successful implementation of the website in daily work practice (Cain \& Mittman, 2002).

Leading theories about the adoption process are dominantly influenced by the work of Rogers (2003), who conceptualizes an individual's decision to adopt or reject a new technology as a process with several stages. The process begins with an awareness of the technology. The individual then forms an attitude towards it based on his/her perception of the technology's characteristics. The individual next decides to either adopt or reject the technology, implements it, and finally confirms his/her decision.

Although a website in itself is not a technological innovation, employing a website as a platform to communicate infection control guidelines in a user-driven manner might imply changes for the individual HCW, but it also implies changes to the work environment and organization in which the website is implemented. Therefore, Rogers' theory can be applied to this thesis's study context. Rogers distinguishes five factors that stimulate the rate of adoption, which below are applied to the adoption process of a website with infection control guidelines. 


\section{Individual factors:}

1) Relative advantage: the degree to which the website is perceived as better than the traditional, paper-based guidelines. The greater the perceived relative advantage of the website, the more rapid its rate of adoption will be;

2) Complexity: the degree to which the website with infection control guidelines is perceived as easy to understand or use will positively affect adoption.

\section{Work-related factors:}

3) Compatibility: the degree to which the website may coexist with current technologies and social patterns. The more compatible the website with infection control guidelines is with current work practice, the higher the prospects for adoption and implementation will be.

\section{Organizational factors:}

4) Trialability: the degree to which users can alter or implement the website on a small scale to try it out without total commitment and with minimal investment;

5) Observability: the extent to which potential adopters can observe the adoption of the website by peers makes it more likely for them to also start using the website.

\subsection{Design framework}

\subsubsection{Basic assumptions}

Given the diagnosis of the problems with existing infection control guidelines, two basic assumptions were made for the construction of a website that can successfully communicate these guidelines:

1) A usable design requires the active involvement of members from the target group;

2) The adoption of a website is a complex, multi-faceted process, influenced by a variety of factors from individual, work-related, and organizational categories.

\section{Active target group involvement}

In order to foster usability of a website featuring infection control guidelines, HCWs should be involved in the development process. In contrast to the usual "top-down" development process of traditional, paper-based guidelines, the communication means should rather be based on a user-driven design process that allows HCWs to clarify their tacit knowledge concerning infection control and empowers them to 
make their own decisions about directions and strategies for improvement (McCoy et al., 2001; Murphy, 2002). In industries such as aviation, nuclear power plants, and electronics, user-driven design is a routine practice. In health care, however, the culture is still to train users (HCWs or patients) to adapt to poorly designed products (Johnson et al., 2005; McCoy et al., 2001; Murphy 2002).

\section{Complexity of the adoption process}

A user-driven website cannot be built and implemented well without understanding how people work and how they will incorporate the website into daily work (Beyer \& Holtzblatt, 1998). Previous studies have identified several factors that may hinder the adoption of web-based guidelines, including the lack of a computer connection in the HCW's office, concerns about the relevance and accuracy of the guidelines on the website, the inability of web-based guidelines to adapt to varied situations, resistance among the ward's management toward web-based guidelines, etc. Jeannot et al., 2003; Rousseau et al., 2003). In other words, the work environment might contain factors impeding website use that have to be considered when implementing the website into daily practice.

Another issue to consider when implementing a website with infection control guidelines in a cross-border context concerns the cultural differences between HCWs from different countries. Since the German culture is characterized by high degrees of uncertainty avoidance and power distance compared to the Netherlands (Hofstede, 1994), German HCWs might hold more positive attitudes towards guideline adherence. Next, German guidelines possibly include more references to law and other legislative regulations (Hofstede, 1994). If these cultural differences appear to explicitly prevail, then both the means communicating the infection control guidelines as well as the implementation strategies should dovetail with HCWs' cultural backgrounds and values.

\subsubsection{Existing user-driven approaches and models}

\section{Approaches}

Three approaches can be distinguished regarding the way in which users are involved in the process of website design (or other technological applications);

1) Participatory design: a set of theories, practices, and studies related to endusers as full participants in activities leading to software and hardware computer products and computer-based activities. The approach is very diverse, and the approach thus has not lent itself to a single theory or paradigm of study or approach to practice (Carroll, 1996; Muller, 2001);

2) User-centered design: a broad philosophy encompassing a variety of methods. Nielsen (1993) adapted and popularized this approach by producing heuristics for usability engineering, which implies more of a focus on usability than on design. Users are not necessarily as involved as full respondents, but they can also serve 
3) Contextual design: user-centered design in the actual context. Contextual design advocates that researchers collect a broad array of data from people in the field where they are living or working, since users are aware of their needs only in the actual context of use (Beyer \& Holtzblatt, 1998). Contextual design comes with a detailed design methodology, leaving little room for issues like available time, budget, and expertise (Holtzblatt et al., 2005).

Since the wide array of available research methods and the flexible user role of the user-centered design approach fit our first basic assumption best, and the environmental focus of the contextual design approach meets our second assumption, we started to search for an appropriate user-driven design model from a bipartite perspective (based both on a user-centered as well as a contextual design approach).

\section{Models}

Papers that report on technological health care applications based on a user-driven approach are abundant. However, few authors reflect on the approach underlying the design process, its phases, and their relationships. One of the exceptions is the study by Thursky and Mahemoff (2007), who apply contextual design for the development of a decision support system for antibiotic prescription. Johnson et al. (2005) formulated a framework that meets all conditions for an effective user-centered design process, based on computer science, cognitive science, psychology, and human-computer interaction. Despite their captivating work, Johnson's model does not guide the development of new websites, instead guiding the redesign process of existing systems.

Also, in none of the identified studies were both basic assumptions mentioned in section 1.2.1. explicitly considered. Authors either claim to have employed a userdriven design approach without engaging real users in their study (Currim et al., 2006; Stevens et al., 2008; Tang et al., 2003; Thursky \& Mahemoff, 2007) or do not consider the factors affecting adoption (Boulos, 2006; Ferney \& Marshall, 2006; Fetters, 2004; Keulers et al., 2006; Scandurra et al., 2008).

Since we could not easily detect studies utilizing a user-driven approach for the design process, either, it was hard to identify a design model that is compatible with the two basic assumptions. A design model is a model that identifies and defines the activities belonging to the design process as well as their interdependencies, sequences, inputs, and outputs.

Despite the availability of a range of user-driven models comparable to the ISO 13407 model (1999) entitled "Human-centered design processes for interactive systems" (see Figure 1.1.), in many of them, the users' input was not consistently incorporated in every part of the process, and most models failed to consider factors affecting the adoption of the system in users' daily practice. 


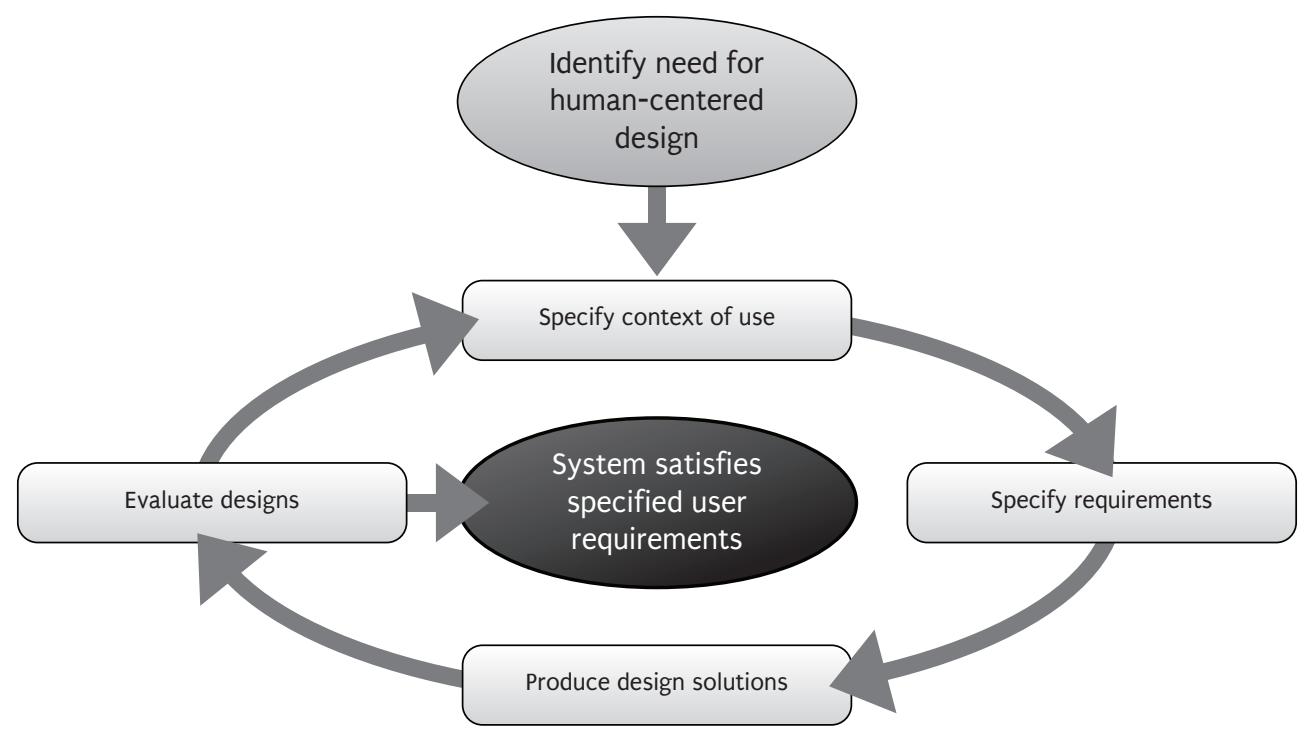

Figure 1.1. Phases in the user-centered design process (International Organization for Standardization, 1999)

\subsubsection{The design model developed by Kinzie et al. (2002)}

One model was identified as appearing to fit the two basic assumptions: the so-called “User-centered model for web site design”, developed by Kinzie and colleagues (2002). This model is based on theoretical literature on usability testing, interface design, and instructional design (Gagne 1985; Norman, 1988; Lynch, 1997; Weinman, 1997). Kinzie's model consists of three phases and seven steps (see Figure 1.2.):

\section{Analysis phase}

1) In step 1, user needs are thoroughly assessed. Needs assessment can be used to explore what is currently occurring and how individuals feel about it, and it can identify potential solutions. Ideally, quantitative and qualitative methods are combined to obtain a comprehensive understanding of the user needs. This step results in a set of specific user needs regarding the website.

2) Step 2 concerns further examination of the needs identified during step 1. Needs are selected and prioritized by the design team. Considerations here are organizational goals, the consequences of needs not being met, and the time, budget, and expertise available for the project. Additionally, barriers and facilitators that users may face are identified. This step ends in the user needs that should necessarily be addressed by the website.

3) Based on the outcomes of step 2, potential solutions are identified in step 3, and their strengths and weaknesses are evaluated by the designers. Subsequently, 
short-term and long-term project goals are drafted to describe what users will be able to do as a function of using the project materials. This step generates a detailed set of the website's functional requirements.

\section{Design phase}

4) The designers start to visualize the website, keeping in mind the goals and tasks formulated in step 3. Storyboards or paper prototypes are created and discussed with users, and as a result of this, the prototype is refined. The design phase ends with the technical realization of the website.

\section{Implementation phase}

5) When the website is completed, it is ready for implementation. The implementation strategy should be carefully selected, depending on goals and tasks, the intended users and their barriers and facilitators for future use. Kinzie et al. do not suggest how to actually perform this.

6) A critical time period for users occurs early in their use of a website; if they perceive that some short-term needs are met in step 6, they are likely to adopt the website in daily practice. Therefore, it is of vital importance to keep users engaged after implementation. The design team should stay continuously in conversation with the users. Kinzie et al., however, do not suggest any methodologies that could be applied here. This step results in barriers to and facilitators of website adoption.

7) Once users have adopted the website and use it, there is a reasonable probability that their long-term needs will be met or, if not met, at least positively influenced. This occurs in step 7 of the design model. Notwithstanding the fact that in this step users have successfully adopted the website, it is essential to ensure that the website stays optimally embedded in the users' environment. This step shows which factors affect the long-term use of the website and the incorporation of the website into daily practice.

Kinzie's model meets the two basic assumptions, first by recognizing the need for continuous user involvement during analysis, design, and implementation of the website. Second, by stressing the need for a rationalized implementation strategy. In other words, the model fits our bipartite approach to user-centered design (flexible user role and customizable methodology) and contextual design (environmental focus). For these reasons, this model was deployed as the guiding framework for the development of a website to facilitate HCWs' adherence to infection control guidelines. 


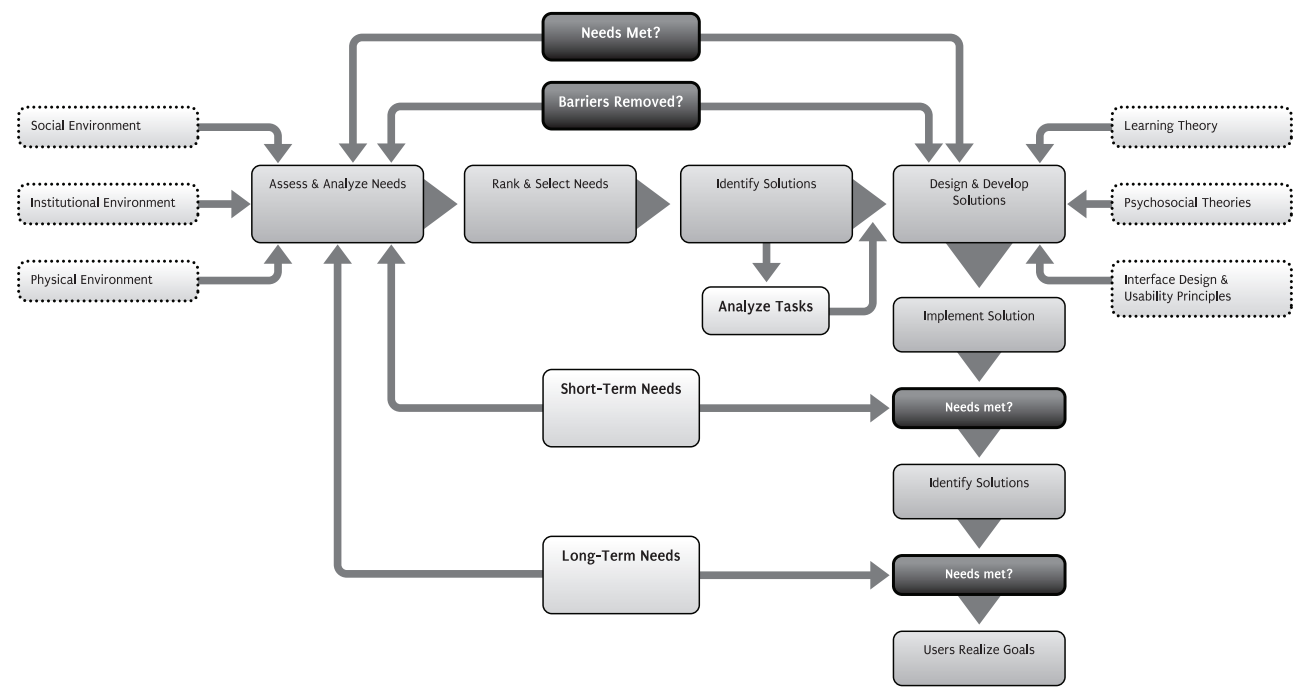

Figure 1.2. User-centered design model for website design (Kinzie et al., 2002)

However, the model has some limitations. First, users are not involved in steps 2 or 3 . Also, it misses clear-cut implementation strategies and methods to assess the degree of user adoption. Therefore, the model was extended with elements from the PRECEDE model, which is explained in the next section.

\subsubsection{Extending Kinzie's model with PRECEDE}

The adoption of a website is not solely determined by its quality and usability, but there are also external factors from individual (e.g., attitude, knowledge, job satisfaction), work-related (e.g., sufficient staff and time), and organizational categories (e.g., communication, training, management values) that must be considered when developing an implementation strategy. Disregarding these factors may limit the technology's usefulness and delay decision-making. Nevertheless, these external factors are usually left out of consideration when implementing websites in health care (Cain \& Mittman, 2002; Kaplan, 1997).

The PRECEDE (Predisposing, Reinforcing, and Enabling Causes in Educational Diagnosis and Evaluation) model recognizes that factors of each of these three categories are interrelated and that each is situated on another hierarchical rank (see Figure 1.3.) (Dejoy et al., 2000; Green \& Kreuter, 2006). While other models assume that the three separate groups of factors only have direct effects on intended behavior, the PRECEDE model suggests that there is also an indirect effect of organizational factors (macro) like management values or interpersonal communication on behavior. Moreover, these effects are mediated by work-related (meso) and individual (micro) factors. For these reasons, the PRECEDE model was deemed appropriate for studying barriers to and facilitators of adoption of the website among HCWs, as well as for studying factors affecting adherence to the guidelines communicated on the website. 
Although PRECEDE is normally used by health planners to direct health education interventions, it has been adapted for the infection control setting (Dejoy, 2000) and has also been presented as an applicable framework for factors affecting technology adoption (Kukafka et al., 2003). To our knowledge, this thesis's research was one of the first attempts to put the latter framework into operation. Furthermore, we complemented the PRECEDE model with a fourth group of factors comprising quality characteristics of health-related websites. This group is not considered in the PRECEDE model but is essential for the successful communication of infection control guidelines: information has to be accurate, complete, readable, and the system should be easy to use (Eysenbach et al., 2002; Kerr et al., 2006).

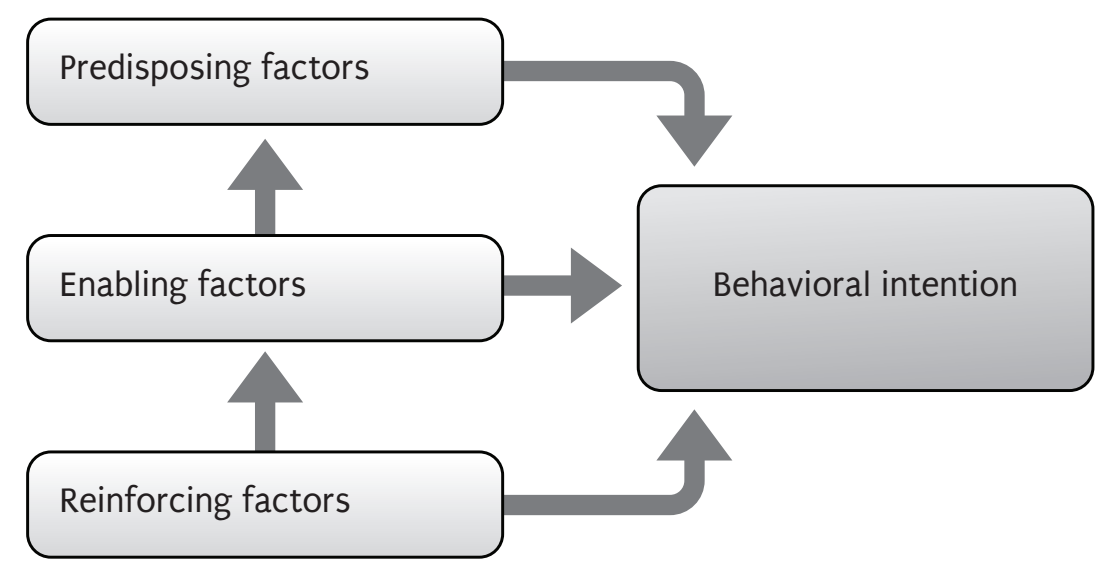

Figure 1.3. PRECEDE model

\subsection{Research questions}

The aforementioned models (Kinzie's user-centered design model and PRECEDE) have been used to design, implement, and evaluate a website that aims to facilitate HCWs' adherence to MRSA guidelines. We investigated to what degree a user-driven approach improved the usability of traditional, paper-based guidelines next to factors affecting adoption of the website in daily practice. Therefore, the overall question of this thesis's research is:

To what degree does user-driven guideline communication enable HCWs to more successfully retrieve and adopt infection control guidelines compared to expertdriven guideline communication? 
Successful retrieval of infection control guidelines implies that HCWs:

- Identify a higher rate of complete, comprehensible, and accurate guidelines when using the website compared to traditional, paper-based guidelines (effectiveness);

- Find infection control guidelines on the website with less time and effort compared to existing traditional, paper-based guidelines (efficiency);

- Are more comfortable with the website and hold more positive attitudes towards it compared to traditional, paper-based guidelines (satisfaction).

Successful adoption of the website with infection control guidelines is achieved when individual and groups of HCWs start using the website for the retrieval of infection control guidelines in daily work practice and adhere to the guidelines communicated on the website.

The overall research question can be divided into five separate research questions. Each question is inherent to a phase of the user-centered model for website design (see Figure 1.2.).

\section{Analysis phase}

1) What are HCWs' needs and demands regarding a website with infection control guidelines?

\section{Design phase}

2) How can a website with infection control guidelines optimally structure, present, and ensure the quality of the guidelines?

\section{Implementation phase}

3) To what degree does a website with infection control guidelines prevent the problems encountered by HCWs when using traditional, paper-based guidelines (or do any new problems arise)?

4) What barriers and facilitators do HCWs perceive according to the adoption of a website with infection control guidelines in daily work practice?

5) What barriers and facilitators do HCWs perceive according to adherence to infection control guidelines communicated on a website?

This thesis serves as an example for both usability practitioners and infection control professionals. For usability practitioners, this thesis shows the crucial methodological steps and the design principles necessary to make health information on the Internet more accessible to HCWs, not only with regard to infection control guidelines but also in other contexts such as chronic diseases. For infection control professionals, this 
thesis's research generated a comprehensive set of factors affecting the adoption of a website with infection control guidelines in daily work practice. Moreover, intervention strategies for guideline adherence are suggested.

\subsection{Thesis outline}

This thesis is comprised of eight chapters. Each chapter, except for the first and the last, can be allocated to one or more steps of the user-driven model developed by Kinzie et al. (2002), which guided the design process of the website.

\section{Chapter 2: Assess and analyze needs}

Chapter 2 describes a content analysis of current, national paper-based infection control guidelines that are recognized as standards of care in the concerned country. Guidelines from the Netherlands, Germany, Great Britain, and the United States were involved in this analysis. This analysis resulted in a framework to communicate infection control guidelines in a user-driven way on a website, according to basic quality criteria (Verhoeven et al., 2007).

\section{Chapter 3: Design and development of the website}

Chapter 3 describes the entire design process of the website. First, the framework presented in Chapter 2 is shortly repeated, as it provided the structure according to which guidelines were presented on the website. Second, the results of scenariobased tests $(n=28)$ with HCWs are reported. These scenario-based tests were administered to detect problems when HCWs use paper-based guidelines as well as to identify specific needs regarding the website. The results of these scenariobased tests are shortly reported in Chapter 3 and more extensively in the first part of Chapter 4. Third, results of a questionnaire are presented that was conducted in order to investigate the factors affecting adherence to the guidelines communicated on the website. The results are only briefly reported in Chapter 3 , since these results are discussed extensively in Chapter 7.

Chapter 3 also discusses two studies that are only described in this chapter. Card Sorting was performed with ten HCWs to ensure that the guidelines on the website were structured in a practical, user-oriented way. Card Sorting is often applied for designing the information structure of a website (Tullis, 2003). Also, two non-working mock-ups of the website were created, based on the results of the content analysis and existing usability design guidelines (Koyani et al., 2006). The mock-ups were presented on a laptop during interviews with 14 intended users who evaluated the prototypes. The respondents gave comments so requirements could be clarified and the website's usability could be optimally adjusted to user needs (Snyder, 1996).

Altogether, the five studies described in Chapter 3 provided the input for the first working prototype of the website (Verhoeven et al., 2008, 2009a). 


\section{Chapter 4: Formative evaluation of the website}

This chapter zooms in on the results of the scenario-based tests discussed briefly in Chapter 3. In this chapter, the scenario-based tests are presented as the first of a two-phase study to determine whether the website actually enabled HCWs to retrieve guidelines more efficiently and effectively compared to traditional, paper-based guidelines. In the first study, $28 \mathrm{HCWs}$ were asked to solve tasks using traditional, paper-based infection control guidelines. In order to detect their informationseeking strategies and problems, respondents were asked to think aloud. Based on the findings, three design principles were developed and served as input for the first working prototype of the website.

In the second study, scenario-based tests were conducted with the identical set of 28 HCWs who participated in study 1 , this time using the website in order to test whether it actually increased the efficiency, effectiveness, and satisfaction with which HCWs retrieve infection control guidelines. The second study also served as a formative evaluation of the website before it was launched online, with the purpose of "test running" various aspects of the website and to verify whether the design team missed any errors (Scriven, 1991) (Verhoeven et al., 2009c; Verhoeven et al., 2009d).

\section{Chapter 5: Implementation of the website}

Chapter 5 concerns the summative evaluation of the website after its implementation, investigated through scenario-based tests with $20 \mathrm{HCWs}$ after the website was launched online. We investigated whether the website enabled HCWs to efficiently and effectively search for relevant information, also for those who may not have sophisticated information-seeking skills. Because HCWs used the "open" Internet to retrieve infection control guidelines rather than only the website under investigation, we could also determine the website's position among other online sources with infection control guidelines. I.e., HCWs employed a "free search" in contrast to the scenario-based tests administered in the design phase, when HCWs searched for infection control guidelines within a closed domain. The study results allowed the formulation of specific design recommendations for the web-based communication of infection control guidelines (Verhoeven et al., 2009e).

\section{Chapter 6: Adoption of the website}

The proven, practical value of the website, demonstrated in Chapter 4 and 5 , is no guarantee for the uptake of the website in daily work practice. Numerous obstacles to the adoption of web-based guidelines in daily work practice remain. In order to identify a strategy that would enhance adoption, intervention barriers and facilitators of website adoption were studied among $20 \mathrm{HCWs}$, reported in Chapter 6. These interviews, guided by the PRECEDE model, disclosed critical impediments and incentives for the use of the website in daily practice, such as important opinion leaders, communication channels, and the relative advantages and disadvantages as perceived by the users (Verhoeven et al., 2009f). 


\section{Chapter 7: Adherence to the guidelines communicated by the website}

A usable and well-implemented website in itself is not enough to enhance HCWs' adherence to the infection control guidelines communicated on the website. In order to change HCWs' adherence behavior, an intervention strategy that takes into account individual, work-related, and environmental factors is required. In Chapter 7, a study is described that encompassed questionnaires $(n=217)$ and interviews $(n=24)$ (based on the PRECEDE model) to identify factors affecting the adherence to infection control guidelines, based on HCWs' perceived barriers and facilitators.

Finally, Chapter 8 provides overall conclusions of the studies presented in this thesis. Also, theoretical, methodological, and practical implications are discussed, and the chapter ends with directions for future research.

Figure 1.4. depicts this thesis's chapters incorporated in Kinzie's user-centered model for website design. Please note that Chapters 2 to 7 are all based on manuscripts that were published in, or submitted to, academic journals. Therefore, there will be some overlap in the content of the chapters, particularly in the introduction and method sections.

\section{Chapter 2}

Assess and analyze needs

\section{Chapters $3 \& 4$}

Rank and select needs

\section{Chapters $3 \& 4$}

Identify solutions, Articulate goals

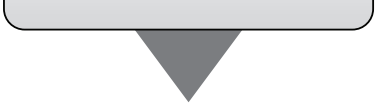

\section{Chapters $3 \& 4$}

Design and development

\section{Chapter 5}

Implement solution

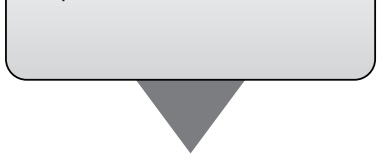

\section{Chapter 6}

Users adopt solution

Chapter 7

Users realize goals

Figure 1.4. Overview of thesis chapters 
As was explained in the previous chapter, infection control guidelines have to serve two hardly reconcilable functions. First, they have to document rules and legislation ("regulation function"), and second, they should serve as a communication means for individual health care workers (HCWs) ("communication function").

To explore the possibilities of how these functions can be integrated, a content analysis of traditional, paper-based guidelines was performed. Paper-based guidelines aimed at the prevention and control of Methicillin Resistant Staphylococcus aureus (MRSA) from Germany, Great Britain, the Netherlands, and the United States that are recognized as standards of care for infection control in their country, were involved in the analysis. German and Dutch guidelines were included due to the Dutch-German context of the project, within which this thesis's research was carried out, and British and American guidelines were selected due to the English language in which they were written and the international reputation of the institutions that provided the guidelines (for instance, Centers for Disease Control and Prevention, Atlanta).

There appeared to be two approaches for presenting MRSA guidelines: (1) Considering guidelines purely as safety regulations by referring to law and including levels of evidence (Germany, Great Britain), and (2) a more user-centered approach with aspects that facilitate the ease of identifying information, such as decision trees, tables, risk categories, referring to information and communication technology, etc. (the Netherlands, United States). In other words, guidelines either emphasized the first function ("regulation function") or the second ("communication function").

However, in order to become fully usable, both functions should be integrated into the communication of infection control guidelines. The content analysis resulted in a framework that can be applied to the communication of infection control guidelines that meets both functions. This framework was used to structure the bilingual website with MRSA guidelines that was developed for the purpose of this thesis's research. 


\section{Chapter 2 \\ Communication of infection control guidelines from a global health perspective: a conceptual framework}

Based on: Verhoeven F, Gemert-Pijnen JEWC van, Friedrich AW, Daniels-Haardt I, Hendrix MGR. Euregionale MRSA-preventie en bestrijding: een vergelijking van Duitse en Nederlandse richtlijnen. [Euregional prevention and control of MRSA: a comparison of German and Dutch guidelines]. Infectieziekten Bulletin 2007:04:125-9. 


\section{Chapter 2}

Assess and analyze needs

\section{Chapters $3 \& 4$}

Rank and select needs

\section{Chapters 3 \& 4}

Identify solutions,

Articulate goals

\section{Chapters $3 \& 4$}

Design and development

\section{Chapter 5}

Implement solution

\section{Chapter 6}

Users adopt solution

\section{Chapter 7}

Users realize goals 


\section{Communication of infection control guidelines from a global health perspective: a conceptual framework}

\subsection{Introduction}

Increased international air traffic and trade in health services have an important role in the rapid spread of infections. The movement of health care workers (HCWs) and patients to supply and access medical services abroad heightens the threat of cross-border disease transmission (Bettcher \& Lee, 2002; Pittet, 2000). With increased patient and HCW mobility, the issue of health care-associated infections (HAIs) should be recognized as a global health problem. Only recently, the World Health Organization addressed the problem by selecting HAls as the topic for its 2005-2006 Global Patient Safety Challenge. A particular focus was the availability and use of clinical practice guidelines on clinical procedures and equipment (World Alliance for Patient Safety, 2005). Some guidelines and documents concerning the development and implementation of clinical practice guidelines are available, but they are limited in scope and in the extent to which they offer concrete tools for national program managers (Pittet et al., 2005). Therefore, we propose a framework in this chapter for the communication of clinical practice guidelines from a global health perspective while taking into account basic quality criteria for clinical practice guidelines.

Clinical practice guidelines approaching HAls as a global health problem should answer two main requirements. First, of course, they should meet basic quality criteria inherent to clinical practice guidelines ("regulation function"), and second, they should elicit a collective awareness among HCWs from different countries to tackle the crossborder transmission of HAls ("communication function").

In order to meet the regulation function, several basic quality criteria should be satisfied (being scientifically valid, provide logistic information, and being reliable). Therefore, we applied the subscales from a framework for the development of practice guidelines having its foundation in industry (ISO gooo) (McRobb, 1990) (see Table 2.1.). Similar quality domains can also be found in the AGREE instrument, the widely accepted standard for assessing the methodological quality and reporting of guidelines (Fervers et al., 2006; The AGREE collaboration, 2003).

However, in order to meet the communication function, it is not enough for clinical practice guidelines to include information about safety regulations: guidelines should be communicated in such a way that they elicit a collective awareness towards 
the problem of HAls among HCWs from different wards, hospitals, and countries worldwide. As with all communicable diseases, an effective prevention policy against HAls implies many moments of communication. For instance, when an infected patient has to be transferred to a healthcare institution abroad, which is becoming more common in our era of global health, it is of vital importance that the receiving institution is aware of the patient's infection status prior to the transfer. To put it differently: HAls can be defined as a collective problem demanding the interaction and collaboration of HCWs from different health care institutions worldwide. Clinical practice guidelines should therefore focus on the tangible aspects of information and communication in order to elicit a collective awareness among HCWs toward the importance of HAls. Additionally, clinical practice guidelines should underline a HCW's individual accountability to the problem of HAls; indeed, non-adherence by only one HCW may lead to an enormous outbreak. By explicitly discussing the risks, health benefits, and dangers of HAls, the HCW becomes more aware of the fact that he or she will be held responsible for his or her clinical practice. It is through such individual feelings of accountability that norms, rules, and customs become effective mechanisms for social control and adherence-rates are improved (Fervers et al., 2003; Smith \& Hillner, 2001). Therefore, we complemented the existing basic quality criteria for clinical practice guidelines by inserting an information and communication domain and a risk-focused domain in order to ensure that clinical practice guidelines also fulfill the communication function next to the regulation function (see Table 2.1.).

In this chapter, we propose a conceptual framework to (1) determine the degree to which current clinical practice guidelines from different countries are already adjusted to a global health perspective concerning HAls (i.e., meet both the regulation and communication function) and (2) to provide a structure for national program managers to communicate infection control guidelines from a global health perspective. Our aim was not to assess the clinical content of the guidelines nor was it to assess the quality of evidence underpinning the recommendation.

\subsection{Methods}

\subsubsection{Selection of clinical practice guidelines}

The cross-border research project MRSA-net Twente/Münsterland, funded by the European Union, is an established group of Dutch and German microbiologists and behavioral scientists to address the problem of HAls at a cross-national level. Methicillin Resistant Staphylococcus aureus (MRSA) served as a starting point in this research project because Germany and the Netherlands substantially vary in MRSArates ( $23 \%$ and $5 \%$, respectively), and thus MRSA threatens the quality of cross-border health care in this region (Deurenberg et al., 2005; Friedrich et al., 2008; Harbarth \& Pittet, 2005; Humphreys, 2006). Therefore, Dutch and German national MRSA guidelines were involved in the analysis, next to guidelines from the Centers for Disease Control and Prevention, Atlanta (CDC), the Society for Healthcare Epidemiology of America (SHEA) in the United States of America (USA), and the guidelines from the Joint Working Party of the British Society of Antimicrobial Chemotherapy, the Hospital 
Table 2.1. Description of basic quality criteria for clinical practice guidelines as applied in industry, adapted for global healthadjusted clinical practice guidelines

Domains for guideline development used Domain adapted for global health-adjusted clinical practice guidelines
in industry*
in industry*

\section{Scientifically valid}

"The guidelines should be based on evidence."

\section{Reliable}

"All documents should be legible, dated, readily identifiable, and maintained in a proper manner."

\section{Scope and purpose}

"The document should clarify its specific target group, function, and primary goal."

\section{Logistics}

“All written procedures should be stated simply, unambiguously, and understandably, and should indicate methods to be used and criteria to be satisfied"

\section{Evidence-based}

The guidelines and its relevance are stressed in the document by means of evidence from multiple sources. This evidence can be scientific proof, but there are also other forms of argumentation that may stimulate the user to adhere to the guidelines.

\section{Focus on legislative regulations}

The guideline document focuses on rules in the broadest sense of the word: The guidelines are supported by references to legislation, rules, protocols or another form of official regulations.

\section{Risk-focused}

The guidelines concentrate on evidence-based risks related to the concerned infectious disease. This can be expressed in several ways, for instance by classifying patients and employees in certain risk groups on basis of which the user must act. Another example considers the consequences of non-adherence to the guidelines.

\section{Information and communication-oriented}

The guideline documentation anticipates the usability of the document: it includes aspects facilitating the ease of identifying information, enhance the comprehensibility of the guidelines or non-textual elements that tell the user what should be done. The guideline document acknowledges the importance of information and communication in an era of increased international trade of health services, e.g., by means of defining tasks and responsibilities, and explicitly mentioning who has to inform who in case of patient transport.

${ }^{*}$ McRobb, 1990 
Infection Society, and the Infection Control Nurses Association in Great Britain (GB). British and American guidelines were selected due to the English language in which they were written and the international reputation of the institutions that provided the guidelines. The British and American guidelines are, to our knowledge, the most cited MRSA guidelines worldwide. We used national guidelines that were recognized as standards of care in the concerned country. The guidelines were identified by searching Google or the website of the responsible authorities. Keywords used were: "guidelines" or "protocol", and "MRSA". We included the most recent guidelines that were available at the time of research (October, 2005). Thirteen national guideline documents originating from four countries and seven different national authorities were included for analysis: GB ( $n=2)$, Germany $(n=4)$, the Netherlands $(N L)(n=3)$, and the USA $(n=4)$ (Centers for Disease Control and Prevention, Atlanta, 2007 (a), 2007 (b), 2007 (c); Coia et al., 2006; Duckworth \& Heathcock, 1995; Dutch Working party on Infection Prevention, 2005 (a), 2005 (b); Muto et al., 2003; National Coordinator Infectious Disease Control, 2005; Robert Koch Institute 2004; The Committee for Hospital Hygiene and Infection Prevention at the Robert Koch Institute, 1999, 2005; The Institute of Public Health, 2003, 2005).

\subsubsection{Framework development and analysis}

Two of the authors (FV, JVG) generated an initial list of items based on validated frameworks for guidelines used in industry and health care (see left column of Table 2.1.) (Fervers et al; 2006 McRobb, 1990; Nabitz et al., 2000; The AGREE Collaboration, 2003). They examined the 13 national MRSA guidelines and explored which guideline aspects could be related to each of the domains representing a global health perspective (see right column of Table 2.1.). During an iterative process of analyzing and discussing, the ultimate framework for global-health adjusted guidelines emerged, which is presented in Table 2.2.

In order to test the content validity of the framework, the two authors independently classified the items according to the definitions of the four domains (see right column of Table 2.1.) and Cohen's Kappa index was calculated (Cohen, 1960). The results revealed that the items in the framework presented a Kappa of 0.77 , implying a substantial inter-rater reliability (Landis \& Koch, 1977). The coding scheme was used to check each of the 13 guidelines for the absence or presence of each item. This was also done independently by the two authors. The results were compared, and the lack of consensus regarding coding differences was solved by discussion.

\subsection{Results}

The analysis of national MRSA guidelines showed differences between the proportions of guidelines in which the items accompanying the four domains were present. Results are shown in Table 2.2. 


\subsubsection{Regulation function}

According to Table 2.2., the British guidelines incorporated substantially more evidence-based items, implying that they used of levels of evidence and included lists of references. Half of the American and German guidelines included all evidencerelated items. The Netherlands showed the least evidence-based elements: none of the three Dutch guidelines contained grades of evidence indicating the strength of scientific data.

Table 2.2. shows that German and British guidelines referred substantially more frequently to regulation and legislation compared to Dutch and American guidelines. An example of such a reference is: "All nursing and residential homes should have a designated member of staff to deal with infection control matters and to ensure that the proprietor's responsibilities to provide adequate arrangements for prevention of infections in the home, under the Nursing Homes and Mental Nursing Homes Regulations 1984, are fulfilled." (Duckworth \& Heathcock, 1995).

\subsubsection{Communication function}

Based on the results, both the British and Dutch guidelines can be labeled as "riskfocused". They both included definitions of risk categories, clinical information such as MRSA rates of the concerning country, and they both emphasized the risk of non-adherence to the guidelines by mentioning negative consequences. The German guidelines also included these risk categories, but they were not as obviously present as in the Dutch and British guideline documents. To illustrate these risk dimensions, a fragment from the British guideline for healthcare institutions is given: "Patients at high risk of carriage of MRSA include those who are: known to have been infected or colonized with MRSA in the past (Category $1 \mathrm{~b}$ ); frequent re-admissions to any healthcare facility (Category 1b); direct interhospital transfers (Category $1 \mathrm{~b}$ ); recent inpatients at hospitals abroad or hospitals in GB which are known or likely to have a high prevalence of MRSA (Category 1b); and residents of residential care facilities where there is a known or likely high prevalence of MRSA carriage (Category 1b)." (Coia et al., 2006). The USA were the only country producing guidelines in which patients and HCWs were not explicitly divided into risk categories.

American guidelines seemed to pay the least attention to information- and communication items, compared to guidelines from other countries. German, British, and Dutch guidelines included schemes and specific recommendations for the information process, in contrast to the American guidelines, which did not. However, the American and Dutch guidelines did refer to the importance of information and communication technology (ICT). For instance: “A hospital computer system can be used to store information regarding long-term isolation indicators for patients known to be colonized with antibiotic-resistant pathogens such as MRSA or VRE. With optimal programming, this can come up automatically whenever the patient enters the healthcare system, whether in the hospital, emergency department, outpatient clinic, or a diagnostic or procedure area, providing an alert to HCWs who may be interacting with the patient for the first time and are unaware of the requirement for isolation." 
Table 2.2. Overview of criteria for the communication of infection control guidelines from a global health perspective, and the degree to which countries' national guidelines meet these criteria

\begin{tabular}{|c|c|c|c|c|c|}
\hline \multirow[b]{2}{*}{ Items } & \multicolumn{5}{|c|}{$\begin{array}{l}\text { Percentage of clinical practice guide- } \\
\text { lines in which item is present }\end{array}$} \\
\hline & $\begin{array}{l}\mathrm{GB}^{1} \\
\mathrm{~N}=2^{*}\end{array}$ & $\begin{array}{l}\mathrm{DE}^{2} \\
\mathrm{~N}=4^{*}\end{array}$ & $\begin{array}{l}\mathrm{NL}^{3} \\
\mathrm{~N}=3^{*}\end{array}$ & $\begin{array}{l}\mathrm{USA}^{4} \\
\mathrm{~N}=4^{*}\end{array}$ & \\
\hline \multicolumn{6}{|l|}{ Domain 1: Evidence-based } \\
\hline 1. Levels of evidence (categories of importance) & 50 & 50 & o & 50 & \\
\hline 2. Information about cost-effectiveness & 50 & 50 & 33 & 50 & \\
\hline 3. Guidelines are supported by scientific evidence (with reference to source mentioned in text) & 100 & 50 & o & 25 & \\
\hline 4. List of references & 100 & 50 & 100 & 75 & .0 \\
\hline \multicolumn{5}{|l|}{ Domain 2: Focus on legislative regulations } & 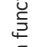 \\
\hline 5. Written in imperative style & o & 75 & 100 & 75 & $\stackrel{\substack{0\\
}}{\underline{0}}$ \\
\hline 6. Reference to other guidelines & 100 & 100 & 100 & 25 & $\begin{array}{l}\overrightarrow{2} \\
\stackrel{2}{\alpha}\end{array}$ \\
\hline 7. Reference to law & 50 & 100 & 33 & o & \\
\hline 8. Date of revision present & ० & ० & 67 & 0 & \\
\hline 9. Information provided about who approved the document & 100 & 100 & 100 & 25 & \\
\hline 10 Names of authors mentioned & 100 & 50 & 25 & 25 & \\
\hline \multicolumn{6}{|l|}{ Domain 3: Risk-focused } \\
\hline 11. Definition of risk categories & 100 & 50 & 100 & o & \\
\hline 12. Clinical information: e.g., prevalence of infectious disease in concerned country is mentioned & 100 & 75 & 67 & 50 & \\
\hline 13. Motivation: Guidelines are supported by mentioning dangers and risks & 100 & 25 & 100 & 75 & \\
\hline 14. Clinical information: Guidelines are supported by mentioning advantages (health gain) & 50 & 25 & 67 & 25 & \\
\hline \multicolumn{6}{|l|}{ Domain 4: Information and communication-oriented } \\
\hline 15. Target group is community & 0 & ० & 33 & 25 & \\
\hline 16. Glossary & 50 & 25 & 100 & 50 & \\
\hline 17. Online available & 50 & 100 & 100 & 100 & 드 \\
\hline 18. Contact details present (for more information) & 100 & 0 & 33 & 25 & 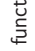 \\
\hline 19. Background information about infectious disease & 50 & 25 & 100 & 50 & 흠 \\
\hline 20. Abstract present in document & 100 & 50 & o & ० & $\stackrel{.0}{\frac{u}{5}}$ \\
\hline 21. Implementation of guidelines: Tools for application & 100 & 50 & 0 & o & 气े \\
\hline 22. Service(s): References to sources for more information etc., appendices, examples of forms & 100 & 75 & 100 & 50 & \\
\hline 23. Education (Is information about education mentioned?) & 100 & 100 & 33 & 50 & \\
\hline 24. Schematizing (decision tree) & 50 & 25 & 100 & o & \\
\hline 25. Question-oriented approach & 50 & 50 & o & 75 & \\
\hline 26. Division of content (according to different phases of infection transmission route) & 50 & 50 & 67 & 25 & \\
\hline 27. Reference to information- and communication technology & o & 0 & 33 & 25 & \\
\hline 28. Logistics: Communication (Who has to inform who in which case?) & 100 & 100 & 100 & o & \\
\hline $\begin{array}{l}\text { 29. Logistics: Identification of tasks, responsibilities, and competences (Who does what and } \\
\text { when?) }\end{array}$ & 100 & 100 & 100 & 25 & \\
\hline
\end{tabular}

${ }^{*} \mathrm{~N}=$ Number of national guidelines included in analysis originating from the concerned country. For example: Two guidelines from Great-Britain were present. 50 implies that in $50 \%$, thus in 1 guideline document, levels of evidence were present.

${ }^{1}$ Great Britain; ${ }^{2}$ Germany; ${ }^{3}$ the Netherlands; ${ }^{4}$ United States of America 
(Muto et al., 2003).

Although all 13 guidelines clearly stated the overall objective, the clinical questions, and the target population of the guidelines, there was one remarkable difference in terms of the scope and purpose of the guidelines: in contrast to the German and British guidelines, one Dutch and one American guideline were aimed at MRSA in the public health setting. The Dutch guideline for MRSA in public health focused on MRSA outside the hospital; MRSA in kindergartens, penitentiary institutions etc. (National Coordinator Infectious Disease Control, 2005). The American guideline for the public considered issues such as "How can I prevent staph or MRSA skin infections?" or "Can I get a staph or MRSA infection at my health club?" (Centers for Disease Control and Prevention, Atlanta, 2007).

\subsection{Discussion}

In this chapter, we introduced a conceptual framework for the design of communication of infection control guidelines from a global health perspective, i.e., reconciling the regulation and communication function that communication of clinical practice guidelines should fulfill. A comparison of national clinical practice guidelines from four countries showed that none of guidelines are completely adjusted to HAls as a global health problem, since none of the guidelines was comprised of all 29 elements from the framework presented in Table 2.2.

From the analysis, there appeared to be two approaches for structuring clinical practice guidelines: (1) considering a clinical practice guideline as a document containing safety regulations by stressing legislative regulations and evidence, as is the case in GB and Germany ("regulation function"), and (2) approaching HAls as a collective management problem, by stressing risks and information and communications items, which is the case in Dutch and American guidelines ("communication function").

In order to meet the requirements of a global health perspective, guidelines should not emphasize one function but integrate both. Guidelines that merely emphasize safety regulations are effective in industry but not in health care. Safety in health care differs in two respects from safety in industry: (1) the patient is at the center of discussion instead of the product, and (2) HCWs are not steps along an assembly line or cogs in a machine producing a product; they are professionals who apply knowledge, adapt learned procedures, and use judgment at each step of the care process, and therefore a mistake made by one HCW may have fatal consequences. By considering that a successful HAls prevention and control policy requires the (crossnational) participation of all HCWs within and between institutions because deviant behavior from just one HCW may lead to an outbreak, and by giving concrete tools how to perform this in practice, clinical practice guidelines can create a form of awareness among HCWs, which is needed in our era of increasing international trade in health services. Adding items like risk categories and decision trees simplifies the decision process for an individual HCW and thereby possibly decreases the numerous 
possibilities to apply knowledge inadequately and make mistakes. The reference to ICT seems to recognize that the only way of jointly solving the MRSA problem is by clear communication between HCWs, and ICT facilitates this by making a network for interaction available. By adding risk- and ICT- related items guidelines, HCWs will become aware of the fact that they are part of a large health care network in which they have to interact in order to prevent and control HAls.

A country's chosen approach for guideline communication might depend on contextual factors such as the organization of care and cultural values. In Germany for instance, great significance is attached to rules and norms in order to avoid uncertainty. This might explain the degree to which German guidelines refer to law and other legislative regulations (Hofstede, 1994).

The poor adherence to infection control guidelines, as described in the literature (Berhe et al., 2005), might be interwoven with the lack of explicit risk- and information and communication items in the guidelines (Van Gemert-Pijnen et al., 2005). It would be interesting to investigate whether the chosen approach for the communication of infection control guidelines is related to the functioning of the guidelines in practice. For instance, when the guideline does not contain obvious risk dimensions, it may be more difficult for a $\mathrm{HCW}$ to ponder risky situations by himself and take appropriate measures. HCWs will start applying their own insights, possibly with dangerous consequences.

If national program managers want to adjust their current HAIs' clinical practice guidelines to a global health perspective, simply copying all the items from the framework is insufficient. The uptake of clinical practice guidelines in practice depends on a country's organizational, legislative, and cultural context (Fervers et al., 2006), such as infection control practices (e.g., use of alcohol-based handrubs for hand hygiene) and infrastructure (e.g., availability of single rooms), and the absence or presence of auditing and feedback activities to monitor the impact of surveillance and interventions (Pittet, 2000).

The framework presented in this chapter was used to structure the guidelines communicated via a bilingual website aimed at the prevention and control of MRSA, developed within the scope of the cross-border research project EUREGIO MRSA-net Twente/Münsterland (Friedrich et al., 2008). Whether the communication of infection control guidelines from a global health perspective is perceived as more usable among HCWs compared to traditional guidelines is a subject for future research. 
The framework presented in the previous chapter can be employed for the communication of infection control guidelines that serves both the regulation and communication function. This framework provided the content and structure of the bilingual website that was developed for the purpose of this thesis's research. However, in order to realize that health care workers (HCWs) can efficiently and effectively retrieve infection control guidelines on this website, user-centered research is required. Therefore, four additional studies were administered to ensure that the structure and presentation of the website dovetail with HCWs' needs: scenario-based tests, questionnaires, Card Sorting Tasks, and prototyping interviews. The results of these four user-centered studies together with the results from the content analysis provided the input for the first working prototype of the website. This chapter describes the contribution of each of these studies to the development of the website.

The first three studies were intended to assess, rank, and select HCWs' needs regarding the website and to articulate the website's goals. The first method involved the content analysis reported in the previous chapter. The current chapter will shortly summarize the most salient outcomes of the content analysis and will show how the framework was translated to the website. Second, scenario-based tests were administered among $28 \mathrm{HCWs}$ to identify problems that HCWs encountered while using existing, paper-based infection control guidelines. Based on the results, user needs for the website were ranked and selected, ending in the website's functional requirements. The results of these tests are briefly described in this chapter, and a more extensive report will follow in Chapter 4. Third, a questionnaire was conducted ( $n=217)$ considering factors affecting adherence to the guidelines that are communicated on the website. This chapter only summarizes the results, since the results are described into more detail in Chapter 7.

The fourth and fifth method concerned the actual design of the website. Two nonworking prototypes of the website were created, based on existing design guidelines for usability and the checklist presented in the previous chapter. The prototypes were presented on a laptop during interviews with 14 intended users who evaluated the prototypes. The respondents raised their comments so requirements could be clarified and the website's usability could be optimally adjusted to user needs. Card Sorting was then employed with ten HCWs in order to determine the website's optimal menu structure.

Overall, this chapter gives a complete overview of the analysis and design phase of the website. 


\section{Chapter 3}

The development of a web-based information tool for cross-border prevention and control of Methicillin Resistant Staphylococcus aureus

Based on: Verhoeven F, Gemert-Pijnen JEWC van, Friedrich AW, Daniels-Haardt I, Hendrix MGR, Steehouder MF. The development of a web-based information tool for cross-border prevention and control of Methicillin Resistant Staphylococcus aureus. International Journal of Infection Control 2008;4:1-11. 


\section{Chapter 2}

Assess and analyze needs

\section{Chapters $3 \& 4$}

Rank and select needs

\section{Chapters 3 \& 4} Identify solutions, Articulate goals

\section{Chapters 3 \& 4}

Design and development

\section{Chapter 5}

Implement solution

\section{Chapter 6}

Users adopt solution

\section{Chapter 7}

Users realize goals 


\section{The development of a web-based information tool for cross-border prevention and control of Methicillin Resistant Staphylococcus aureus}

\subsection{Introduction and background}

Increased international air traffic and trade in health services have an important role in the rapid spread of infections. Movement of health care workers (HCWs) and patients to supply and access medical services abroad heighten the threat of crossborder disease transmission (Bettcher \& Lee, 2002; Pittet et al., 2005). The occurrence of health care-associated infections (HAls) like Methicillin Resistant Staphylococcus aureus (MRSA) is detrimental both to patients' and HCWs' safety and, consequently, to quality of care (Pittet \& Donaldson, 2006). This threat particularly holds for European borders, when patients and HCWs are transferred from a middle- or high-endemic country, such as Germany, to a low-endemic country, such as the Netherlands, with an MRSA prevalence of $23 \%$ and $5 \%$, respectively (Friedrich et al., 2008; Pittet et al., 2005).

In order to ensure patient safety and to equalize quality of care in cross-border health care settings, prevention of HAls need to be structurally addressed by synergic efforts of the health care actors representing the complete chain of care, on both sides of the border. An example of such an effort is the Dutch-German EUREGIO project "MRSAnet Twente/Münsterland". Since its launch in 2005, this project has founded a crossborder network of the major health care providers (e.g., hospitals, labs, public health services, general practitioners) that jointly developed an effective infection control strategy including effective surveillance, early detection, and better education about HAls for both HCWs and patients, including a website with infection control guidelines. This chapter focuses on the design process of this particular website.

The Internet has evolved as a potentially useful tool for guideline education, dissemination, and implementation because of its open standards and its ability to provide concise, relevant clinical information at the location and time of need. Previous research showed that HCWs' adherence might be better with electronic guidelines than with paper guidelines when tested in clinical scenarios (Thomas et al., 1999). On top of the advantages the Internet has to offer, websites have the potential to combine information to relevant activities by automation of the information seeking and retrieval processes to provide high-quality and appropriate answers to clinical questions. Websites have been implemented for several disease models: asthma (Thomas et al., 1999), low-back surgery (Jeannot et al., 2003), upper 
and lower digestive endoscopies (Jeannot et al., 2003), and infectious diseases such as tuberculosis preventive therapy (Thomas et al., 1999), and hepatitis C (Hedin et al., 1999). However, to our knowledge, a website with guidelines particularly aimed at the prevention and control or MRSA in particular did not yet exist.

In order to ensure that the website's functionalities optimally met HCWs' needs, we applied a user-driven design approach (Kinzie et al., 2002). This chapter describes the steps that appeared to be crucial for the development of a website that aims to provide HCWs with complete, comprehensible, and accurate MRSA guidelines that enhance the decision making process to deliver safe health care.

\subsection{Methods}

\subsubsection{Respondents}

Study respondents involved nurses and physicians from two Dutch and two German general hospitals in the EUREGIO Twente/Münsterland, varying in size from 314 to 1500 beds. A random sample involving HCWs from different types of wards with varying risk management problems was selected by the researcher (intensive care unit, surgery, internal medicine, and paediatric ward). All data were collected between March 2006 and May 2007. Table 3.1. shows the research methods that were successively employed during the analysis and design process of the website.

\subsubsection{Design team}

The website was developed by the interdisciplinary project team of EUREGIO MRSAnet, comprised of medical microbiologists, infection control nurses, physicians from municipal health services, behavioral scientists, application developers, and interface designers from both Germany and the Netherlands.

\subsubsection{Needs assessment}

We performed a needs assessment through a deep qualitative analysis of HCWs' needs and their current activity and work habits to ensure that the website can be successfully integrated in daily work practice (Kinzie et al., 2002). The needs analysis included three stages. We started with systematically analyzing which information is communicated in current Dutch and German national MRSA guidelines, and how this information is presented. This systematic analysis provided us with a structure according to which we could present the guidelines on our website (see Chapter 2 for an extensive description). Subsequently, scenario-based tests were employed among $28 \mathrm{HCWs}$ to identify user requirements for the website. Scenario-based tests involve users performing simulated tasks based on clearly defined scenarios. This technique has previously been established and used successfully to test clinical decision-making (Kolbe et al., 1996; Van Gemert-Pijnen et al., 2005). By detecting and diagnosing problems that occurred with HCWs applying paper-based MRSA guidelines in practice, we were able to determine the discrepancy between the desired and the 
Table 3.1. Overview of the analysis and design process of the website with guidelines aimed at the prevention and control of Methicillin Resistant Staphylococcus aureus

\begin{tabular}{|c|c|c|}
\hline Study & Objective & Procedure \\
\hline a. Document analysis & $\begin{array}{l}\text { Ensure that website adequately } \\
\text { presents useful, resource-rich guide- } \\
\text { line information to the intended } \\
\text { users }\end{array}$ & $\begin{array}{l}\text { Based on literature, a framework for cross-border guidelines } \\
\text { was developed. Two researchers independently coded national } \\
\text { Dutch and German guidelines according to the framework. Re- } \\
\text { sults were compared and consensus about coding differences } \\
\text { was reached by discussion (see Chapter } 2 \text { for an extensive } \\
\text { description). }\end{array}$ \\
\hline b. Scenario-based tests & $\begin{array}{l}\text { Explore usability problems that } \\
\text { occur with paper-based guideline } \\
\text { use, and thereby identification of } \\
\text { user needs }\end{array}$ & $\begin{array}{l}\text { Respondents ( } n=28 \text { ) were asked to verbalize thoughts and } \\
\text { actions (think aloud) while using the guideline to solve an } \\
\text { MRSA-related problem: e.g., "You are attending to an MRSA } \\
\text { patient in an isolation room. Using the guidelines, can you say } \\
\text { aloud which safety preventive guidelines you must take?" (see } \\
\text { Chapter } 4 \text { for an extensive description). }\end{array}$ \\
\hline c. Questionnaire & $\begin{array}{l}\text { Determine how adherence to guide- } \\
\text { lines communicated on website in } \\
\text { clinical practice can be enhanced }\end{array}$ & $\begin{array}{l}\text { Confidential, self-administered close-ended questionnaires } \\
\text { (based on existing instruments fitting the study themes) with } \\
\text { return envelopes were distributed to a random sample of } 320 \\
\text { HCWs. After check for completeness, final sample size was } \\
\mathrm{n}=217 \text {. All data were processed in SPSS } 16.0 \text {. Structural Equa- } \\
\text { tion Modeling techniques (AMOS } 5 \text { Software) were used to } \\
\text { identify intervention strategies. (see Chapter } 7 \text { for an extensive } \\
\text { description). }\end{array}$ \\
\hline d. Prototype interviews & $\begin{array}{l}\text { Validate user requirements and } \\
\text { allowance of users to contribute to } \\
\text { website design }\end{array}$ & $\begin{array}{l}\text { While being shown the mock-ups of the website on a laptop, } \\
\text { respondents ( } n=14 \text { ) commented on the look-and-feel, ex- } \\
\text { pectations, and usability aspects such as credibility, lay-out, } \\
\text { comprehensiveness, etc. Mock-ups consisted of a home- and a } \\
\text { search page. Comments were categorized and quantified. Most } \\
\text { frequently mentioned comments were taken into account in } \\
\text { design revisions of the website. }\end{array}$ \\
\hline e. Card Sort Study & Design website's menu structure & $\begin{array}{l}\text { Respondents }(n=10) \text { sorted cards with content items (ques- } \\
\text { tions) for the website, and labelled the groups they created. } \\
\text { Hand sorted card data were entered into WebSort } \\
\text { analyzed with IBM's EZ sort application, which visualizes the } \\
\text { differences and similarities between items in a tree-diagram } \\
\text { based on cluster analyses. Besides, a qualitative analysis of the } \\
\text { labels and the interviews was conducted. }\end{array}$ \\
\hline
\end{tabular}


perceived guideline information and the guideline's usability perceived by HCWs (see Chapter 4 for an extensive description). Finally, we completed the needs assessment with a questionnaire among $217 \mathrm{HCWs}$ to determine how adherence to the guidelines communicated on the website could be enhanced (Dejoy et al., 2000) (see Chapter 7 for an extensive description).

\subsubsection{Goal articulation}

The needs identified during the needs assessment phase were evaluated by the EUREGIO MRSA-net Twente/Münsterland project team, in order to select and prioritize those needs to be addressed. The issues taken in consideration included the objectives of the EUREGIO MRSA-net project, the consequences of the needs not being met, the available time, budget, and expertise for the EUREGIO-project, and the relation to other project activities. The goals of the website were articulated during a project discussion meeting. Subsequently, we determined based on both literature and experience, what HCWs need to know or be able to do to achieve these goals, which resulted in the website's functional requirements. The website's goals and functional requirements are shown in Table 3.2. in the Results section of this chapter.

\subsubsection{Design and development}

Once the goals and requirements were understood, the actual design process started. We attempted to visualize the website by creating mock-ups and developed a blueprint for the website. Two mock-ups of the homepage and the search page were created, considering common guidelines for web design and usability (Koyani et al., 2006). We evaluated the prototypes by interviewing 14 intended users. Users are in a unique position to provide early, authentic feedback. They know what they need and want and can respond to the design.

The structure of the information provided by our website was a design challenge of particular concern. To ensure a practical use of the website by HCWs, the website had to present very complex action oriented information in a clear and simple a manner, with a very easy navigational structure. To guarantee this, we applied a Card Sort Study among ten HCWs. Card sorting is often applied as a user-centered method for designing the information structure of a website (Tullis, 2003). The initial scenario-based tests (see above) had provided us with 165 key questions that were unanswered by the current MRSA guidelines, while their answers are nevertheless required for HCWs to deliver safe health care. During the Card Sort Study, ten respondents sorted these 165 questions into meaningful groups that eventually served as the website's navigation structure. Hand sorted card data were entered into WebSort ${ }^{\mathrm{TM}}$ tool, and analyzed with IBM's EZ sort application, which visualizes the differences and similarities between items in a tree-diagram based on cluster analysis, providing the website's optimal navigation structure. Besides, a qualitative analysis of the labels and the interviews was conducted to assign names to the categories. 


\subsection{Results}

In this section, the different steps of the website's development process will be successively presented. First, the problems recognized during the needs assessment will be elucidated. Based on the needs assessment, the website's goals were formulated which led to the website's functional requirements, which can be found in Table 3.2. Eventually, we took into account these functional requirements when designing the actual website. Before the actual launch of the website, we validated the website's design and content among the intended users.

\subsubsection{Needs assessment}

\section{a. Document analysis to ensure that website adequately presents useful, resource-rich guideline information to the intended users (see Chapter 2)}

We systematically analyzed existing Dutch and German national MRSA guidelines, in order to determine how the information on our website could best be presented to the target group. Two approaches for structuring MRSA guidelines emerged from this analysis: ( 1 ) Considering guideline documentation as a document containing safety regulations by stressing legislative regulations and evidence, as was the case in Germany ("regulation function"), and (2) approaching the control of MRSA as a collective management problem, by stressing risks, information- and communicationitems, which occurred in Dutch guidelines ("communication function"). We integrated these two approaches in our website by presenting the information as such that both safety regulations and tangible information and communication aspects were present, complemented with practical information from everyday work practice (see Table 3.2.). By including both approaches we represented a global health perspective and strived to enhance that by using the website, HCWs become aware of their role in a large health care network in which they have to interact in order to prevent and control MRSA. This is particularly relevant in cross-border health care settings, where HCWs from different institutions in various countries have to cooperate.

\section{b. Scenario-based tests to explore usability problems that occur with paper- based guideline use, and identification of user needs (see Chapter 4)}

HCWs from both countries encountered several problems while using the paper-based guidelines because the guidelines required them to apply knowledge outside their current knowledge base. $49 \%$ of scenarios that respondents were asked to solve by means of the paper-based guidelines, could not be completed successfully, due to the following causes:

1) Incomplete information (31\% of cases): For instance, the guidelines did make clear which protective clothing should be worn when entering an isolation room, but did not elucidate in which order the clothing should be taken on and off.

2) Mismatch problems ( $28 \%$ of cases): Respondents could not retrieve relevant 
Table 3.2. Identified goals and functional requirements of the website with guidelines aimed at the prevention and control of Methicillin Resistant Staphylococcus aureus, based on needs assessment comprised of three studies

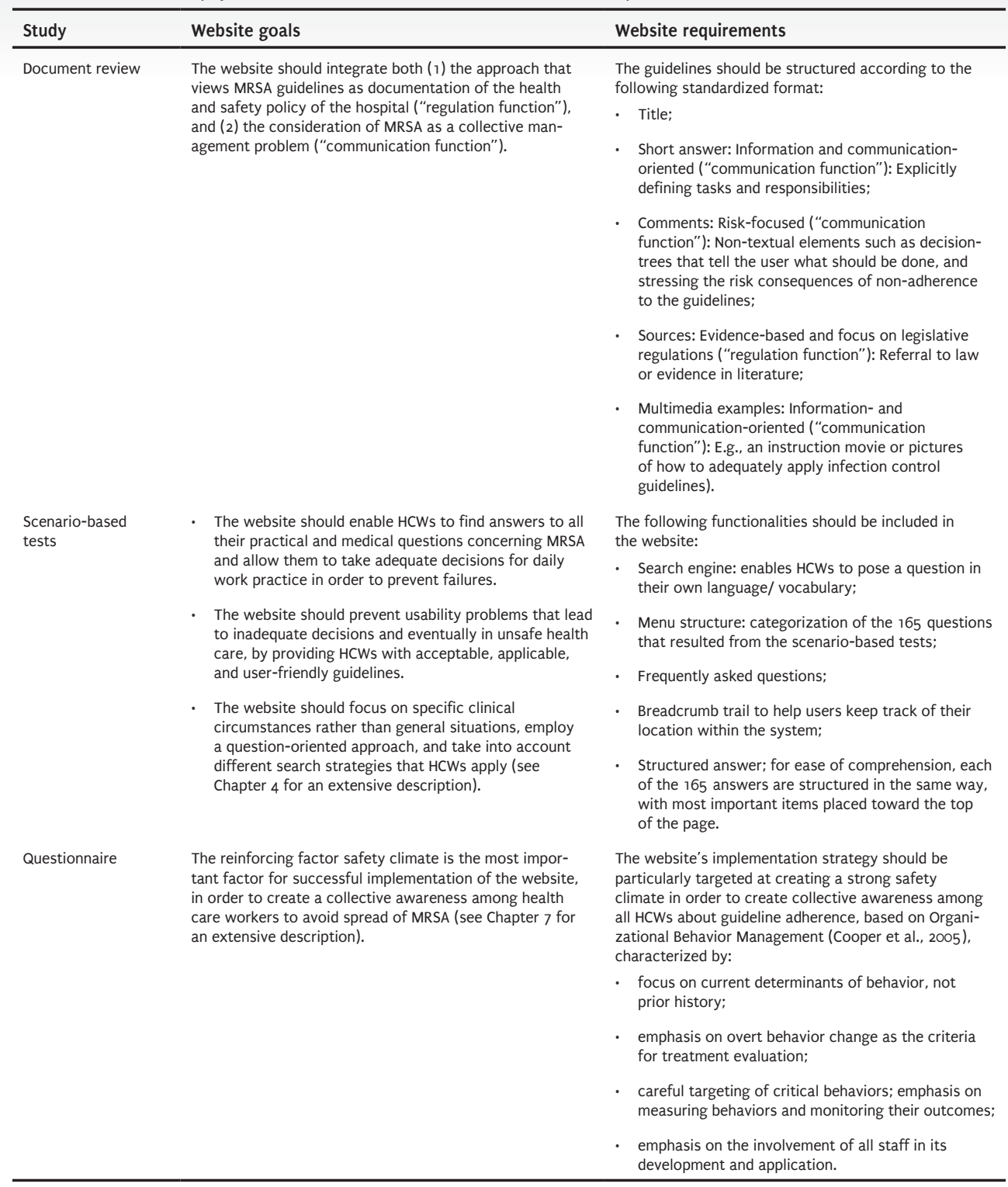


information because of the size of the guidelines and a mismatch between the search terms used by HCWs (e.g., "treatment") and the vocabulary applied in the guidelines (e.g., "decontamination procedure").

3) Incomprehensible information ( $13 \%$ of cases): HCWs appeared to be unfamiliar with the jargon used in the guidelines.

4) Inaccurate information ( $13 \%$ of cases): Outdated information, and a discrepancy between the guideline's rules and the possibilities of performing them in practice. E.g., the guideline requires each (suspicious) MRSA-positive patient to be treated in preventive isolation, while on particular wards no isolation facilities are available.

5) Inadequate information structure ( $15 \%$ of cases): For instance, HCWs expected to find the required information in a particular section of the guidelines or by employing a specific search strategy, but the opposite appeared to be true.

We intended to prevent these problems by tailoring the website's functional requirements to the identified problems (see also Table 3.2.):

1) Incomplete information: the majority of problems were due to incomplete information. In total, 165 key questions relating to everyday work practice were found, for which the guidelines did not provide an adequate answer. Because we strived to provide HCWs with complete, comprehensible, and accurate guidelines that enable them to deliver safe health care, we included the answers of these 165 questions in the website's content. Answers were based on national MRSA guidelines (Dutch Working Party on Infection Prevention, 2005; The Committee for Hospital Hygiene and Infection Prevention at the Robert Koch Institute, 1999), and were complemented and actualized by the Dutch and German medical microbiologists involved in the research project.

2) Mismatch problems and incomprehensible information: In order to communicate the guidelines' content to dovetail with HCWs' vocabulary, we used words on the website such as "get rid of MRSA" instead of "eradication therapy", "take swabs" instead of "perform screening cultures", "outbreak" rather than "epidemic situation", etc.. A dedicated content management system enabled the project team to add and delete keywords to the search engine's database that aid in matching system with HCWs' vocabulary.

3) Inaccurate information: Revision dates and the latest news were included in order to keep information accurate.

4) Inadequate information structure: Each of the 165 additional key questions was answered according to a standardized format based on usability guidelines (Koyani 
et al., 2006) and a content analysis of existing guidelines to meet both regulation and communication functions (see Chapter 2), with important items placed consistently at the top center. In order to make the search process more efficient, the system should allow the user to rapidly switch from one search strategy to another and users should always be able to keep track of their location within the system. Therefore, answers to questions were retrievable through a search engine, a menu structure with categories, and frequently asked questions. We also incorporated a breadcrumb trail. The incorporation of three search options and a breadcrumb trail enabled HCWs to find the relevant information more rapidly with less effort (i.e., more efficient).

\section{c. Questionnaire to determine how to implement website into practice (see Chapter 7)}

The questionnaire results showed that in order to enhance guideline adherence, the hospital's safety climate is a paramount reinforcer. Respondents indicated that in a firm safety climate, risks, health benefits, and dangers of multi-resistant organisms are explicitly discussed. Therefore, these risk elements were also actively advocated on the website in order to increase HCW's awareness of the risk to acquire or transmit MRSA in case of non- adherence to the guidelines. Embedding the website in the hospital's safety climate, e.g., by means of training, might improve guideline adherence.

\subsubsection{Goal articulation}

Potential solutions to the problems recognized by the needs assessment were identified and project goals were drafted by the project team of EUREGIO MRSA-net Twente/Münsterland to describe what users should be able to do as a function of using the website (see Table 3.2.). Goals were then broken down into the website's functional requirements (see also Table 3.2.).

\subsubsection{Design and development}

\section{d. Screen mock-up interviews to validate user requirements and allow of users to contribute to website's design}

We applied research-based web design and usability guidelines (Koyani et al., 2006) when creating two non-working mock-ups. The mock-ups each consisted of a homepage and a search page (see Figures 1, 2, 3, and 4 in the middle of this thesis). When asked to indicate their preference for one of the two mock-ups, 12 out of 14 respondents chose prototype 1, particularly because of "the convenient structure" $(n=3)$, "the topic MRSA is presented clearly" $(n=4)$, and "the trustworthiness suggested by the logos of the involved organizations" $(n=2)$. Prototype 2 was evaluated as "unattractive" ( $n=10)$, "too commercial $(n=7)$ ", and "inconveniently arranged" $(n=6)$. Hence it was decided that the final lay-out of our website should be based on prototype 1 . 


\section{e. Card Sort Study to design information structure of website}

The Card Sort Study resulted in ten categories according to which the 165 questions that resulted from the scenario-based tests could be structured. The categories, each with an example to illustrate the practical approach of the website, are presented in Table 3.3. Compared to the general tables of content of MRSA guidelines, it is remarkable that the search structure based on the Card Sort Study included a category on "MRSA and your own health", and a category on "Communication". We also found that the categories can best be ordered according to the stages of the patient's presence in the hospital: "Admission", "Transport" and "Discharge". It thus appeared that HCWs desired a more action- and communication-oriented way of structuring information than is the case in current Dutch and German MRSA guidelines. By employing a Card Sort Study, we tried to overcome problems with inadequate information structure. Since the categories' names were generated by the respondents themselves, problems caused by a mismatch in vocabulary and incomprehensible information were also prevented.

\subsubsection{System description of the final website}

The results from each of the five studies conducted during the development process were integrated in a final version of the website. Figures 5 and 6 in the middle of this thesis show the final version of the homepage and an answer page. The 165 questions that emerged from the scenario-based tests served as input for the website's content and were answered based on national MRSA guidelines, and complemented by the Dutch and German medical microbiologists involved in the research project. Figure 5 in the middle of this thesis shows how we took into account the different search strategies that users apply by facilitating the website with three different search options. The answers were directly communicated to the user in an instructional, imperative style. The users were addressed directly, e.g., "You first have to put on gloves, followed by a gown, etc." The answers were structured according to usability guidelines, with important items placed consistently at the top center, scroll stoppers were avoided as much as possible, moderate white spaces were used, and feedback was provided on users' location on the website by means of a breadcrumb trail on top of the page, to enable them to keep track of their location within the system (Koyani et al., 2006). The structured answer format that emerged from the content analysis of traditional, paper-based guidelines is visible in Figure 6 in the middle of this thesis.

The website allowed users to decide upon the level of detail of the answer they wish to obtain, because next to the short answer, in-depth elaborations were provided, next to sources (e.g., scientific literature, newspaper articles, links to other websites for further reading, etc.). If possible, multimedia examples (e.g., instruction movies and pictures of how to adequately apply personal protective equipment) were added as well. Furthermore, a specific machine learning functionality was incorporated in the website (Tigelaar et al., 2009). This function enabled the higher ranking of answers to questions that have been looked up most frequently.

A dedicated content management system enabled the project team to add and 
Table 3.3. Categories resulting from the Card Sort Study $(n=10)$, each with an exemplary question

\begin{tabular}{|c|c|}
\hline Category & Question example \\
\hline 1. MRSA in general & Does MRSA threaten the future? \\
\hline 2. Screening & $\begin{array}{l}\text { Sometimes, screening cultures are taken among HCWs in case of a hospitalized MRSA-patient, but } \\
\text { sometimes not. Why is there no consequent screening policy? }\end{array}$ \\
\hline 3. Protective measures & In which order should I put on and off personal protective equipment? \\
\hline 4. Disinfection measures & $\begin{array}{l}\text { How often should I disinfect medical equipment and medical instruments that are used in an isolation } \\
\text { room? }\end{array}$ \\
\hline 5. Admission & Which measures should I take when an MRSA-positive patients enters the emergency room? \\
\hline 6. Transport & $\begin{array}{l}\text { An MRSA-positive patient should be transported from one ward to another within the hospital. Which } \\
\text { personal protective equipment should I wear when accompanying this patient? }\end{array}$ \\
\hline 7. Discharge & $\begin{array}{l}\text { Which materials may I throw away after discharge of an MRSA-positive patient from an isolation } \\
\text { room? }\end{array}$ \\
\hline 8. Treatment & What are the side-effects of eradication therapy? \\
\hline 9. MRSA and your own health & Am I allowed to receive visitors at home, when I am colonized or infected with MRSA? \\
\hline 10. Communication & There is an MRSA-outbreak at my ward. Who should I inform about this and what should I exactly tell? \\
\hline
\end{tabular}


delete questions to the website at any time and location. This system allowed also to continually update the website's content, which is of vital importance because infection control policies are frequently subject to changes. The content management system also permitted the project team to access the logfiles that record all activity that occur on the website. The logfiles record standard data such as page views, session duration, IP addresses, results of the feedback function, and also the queries users entered in the search engine. Questions that were not answered yet by the website could be easily identified by the logfiles, and the project team could immediately add the question and its answer to the website via the content management system.

\subsection{Discussion}

This study provided us with a prototype of a website with guidelines aimed at the prevention and control of MRSA. The different methods that were employed complemented each other in order to formulate the website's goals and requirements. Whereas the content analysis (presented extensively in Chapter 2) provided the structure according to which guidelines should be communicated on a website, the scenario-based tests showed insight in real user behavior and allowed us to better dovetail the website's functional requirements to HCWs' needs and tacit knowledge (see Chapter 4 ). The website's functionalities were specifically designed to prevent the five types of problems that HCWs encountered while using the traditional, paper-based guidelines. Moreover, the questionnaire demonstrated which goals and functional requirements the website should meet in order to fit within the organizational context (see Chapter 7).

A benefit of the employed user-driven approach is that it enhanced HCWs' involvement in the development process, which is often neglected in guideline development of clinical practice guidelines (Van Gemert-Pijnen et al., 2005). Target group involvement is important to create ownership and to foster the applicability of the guidelines, and for the eventual adherence to guidelines (McCoy et al., 2001).

In our study, the Internet enabled us to communicate guidelines to different occupational groups, taking into account different levels of detail various occupational groups are interested in, and different search strategies users maintain. In this way, the information will have the maximum impact on knowledge, attitudes, and subsequent safety behavior (Madle et al., 2004; Sinkowitz-Cochran et al., 2000). Other features of web-based clinical practice guidelines systems are the potential for effective dissemination and free availability at the location and time of need (Thomas et al., 1999). Because respondents in our study indicated that they had a rather practical information need, we tried to couple information in the website to relevant activity. By ensuring that the information is there when, where and how HCWs want it, we expected that good quality information forms the basis for HCWs to make informed choices about their actions, which is called Information Therapy (Gwinn \& Seidman, 2007). Because our tool is web-based, and computer and Internet facilities are available at practically every hospital ward nowadays, the website can be 
incorporated in the workflow of clinical care (Thomas et al., 1999). This is a subject for future research.

Adherence to MRSA guidelines is of vital importance, because the occurrence of multiresistant microorganisms is detrimental both to the patient and HCWs' safety and, consequently, to quality of care (Pittet et al., 2005). Previous research showed that computer-based guideline systems have been shown to improve prescribing practices, reduce serious medication errors, enhance the delivery of preventive care services, and improve adherence to recommended care standards (Kawamoto et al., 2005). In future research, the website's contribution to a higher adherence to MRSA guidelines and eventually a reduction in MRSA rates should be investigated.

\subsubsection{Limitations}

Limitations of our study were the relatively small sample size and limited geographic region, implying the impossibility of generalizing the results to all hospitals in Germany and the Netherlands. Also, due to the voluntary participation of respondents, the results could have been subject to several response biases. HCWs with strong motivations may have been more likely to participate, and there is a concern regarding socially desirable responses.

Another limitation of our study was the laboratory setting, in that behaviors in the pursuit of actual clinical knowledge in a real clinical setting may be different from those shown in this controlled environment (Nielsen, 1993). This concern particularly holds for the questionnaires and the scenario-based tests. However, the ability to use a defined set of scenarios provides a benefit that cannot be obtained in the real clinical setting.

Solely developing a usable website that provides HCWs with infection control guidelines is not sufficient to enhance adherence to guidelines and eventually improve quality of care. For this, it is of vital importance to disseminate and implement the website successfully in the health care setting. Organizational and managerial support as well as systems for audit and evaluation are key elements in the successful implementation of guidelines in practice within the medical profession (Grimshaw \& Russell, 1993). But also the fact that the tool is web-based might cause implementation problems, such as HCW reluctance toward technology, proprietary interests, technical limitations, and local practice environments. The main limitations of the Internet include technical aspects imposed by the open standards and the challenge of controlling information quality (Thomas et al., 1999). These limitations, however, do not necessarily entail disadvantages. The open standards available on the Internet allowed us to simplify our user interface within existing standards and thus ensure widespread availability and ease of navigation. To target the largest possible audience, and to achieve a high degree of evidence, we used nationally developed and widely applied clinical practice guidelines. 


\subsubsection{Future research}

In order to test whether the website actually prevented the problems that HCWs encountered while using the paper-based guidelines, another study consisting of scenario-based tests was performed. The same $28 \mathrm{HCWs}$ completed tasks identical to those of the scenario-based tests with paper-based guidelines, but this time used the website. This round of scenario-based tests also served as a formative evaluation of the website before it was launched online, with the purpose of "test running" various aspects of the website and to verify whether the design team did not miss any errors (Scriven, 1991). The results of this formative evaluation are presented in Chapter 4.

On February 19 2008, the website was implemented (www.mrsa-net.nl). Then, a summative evaluation was performed in order to determine the website's position among other relevant online sources (see Chapter 5 ). Also, a qualitative study was administered to determine factors affecting successful adoption of the website in daily practice, which are reported in Chapter 6.

Since MRSA is becoming ever more prevalent outside the hospital (Van Loo et al., 2007; Voss et al., 2005), we also created a website for patients and the general public according to the same user-driven design process (Verhoeven et al., 2007, 2009b). This website can empower consumers of health services, enabling them to make informed choices (Woolf et al., 2000). This is a subject for future research. 
This chapter describes the results of the website's formative evaluation. First, this chapter zooms in on the results of the scenario-based tests with traditional, paperbased guidelines discussed briefly in Chapter 3. In this chapter, the scenario-based tests are presented as the first of a two-phase study to determine whether the website actually enabled health care workers (HCWs) to retrieve guidelines more efficiently and effectively compared to traditional, paper-based guidelines.

In the first study, $28 \mathrm{HCWs}$ were asked to solve related scenarios using traditional, paper-based infection control guidelines. In order to detect their information-seeking strategies and problems, respondents were asked to think aloud. Usability problems occurred due to poorly structured information (15\% of cases), insufficient quality of information (57\% of cases), and a mismatch between experts' and HCWs' vocabulary (28\% of cases). To overcome these shortcomings, three design principles were formulated for web-based communication of infection control guidelines: better navigation (the guidelines should be searchable in several ways), multimodality (the guidelines should not be presented as text only), and action orientation (the guidelines should be presented as HCWs' behaviors). These design principles were taken into account during the website's development.

In the second study, the same $28 \mathrm{HCWs}$ completed tasks identical to those of the first study while thinking aloud, this time using the website. The percentage of correctly completed tasks increased significantly (Paired Samples T Test, p<.01), and the mean time for task completion decreased significantly (Paired Samples T Test, p<.05). Also, respondents were more satisfied with the website than with the paper-based guidelines. The number of problems due to poor information quality and a mismatch in vocabulary declined, although the number of structural problems increased. This can probably be explained by the fact that the navigation structure was user-driven (using Card Sorting; see Chapter 3), in contrast to the standardized answer format that was based on usability design guidelines and the content analysis reported in Chapter 1 , where no users were involved.

The inadequacies that were detected during the scenario-based tests in study 2 were fixed before the website was officially implemented. 


\section{Chapter 4}

\section{From expert-driven to user-oriented}

communication of infection control guidelines

Based on: Verhoeven F, Steehouder MF, Hendrix MGR, Gemert-Pijnen JEWC van. From expert-driven to user-oriented communication of infection control guidelines. International Journal of Human-Computer Studies 2009. doi:10.1016/j.ijhcs.2009.07.2003 


\section{Chapter 2}

Assess and analyze needs

Chapters $3 \& 4$

Rank and select needs

Chapters $3 \& 4$

Identify solutions, Articulate goals

Chapters 3 \& 4

Design and development

\section{Chapter 5}

Implement solution

\section{Chapter 6}

Users adopt solution

\section{Chapter 7}

Users realize goals 


\section{From expert-driven to user- oriented communication of infection control guidelines}

\subsection{Introduction}

Health care-associated infections cause thousands of preventable deaths each year (Pittet \& Donaldson, 2006). Therefore, it is crucial that health care workers (HCWs) adhere to infection control guidelines. The purpose of infection control guidelines is to educate HCWs about the direction on the prevention and control of infectious diseases and uphold standards of safe work practice. Although most HCWs are aware of the rationale for infection control practices, adherence is generally poor (Berhe et al., 2005). In addition to contextual reasons such as negative management values and a high workload, the insufficient tailoring of infection control guidelines as a communication means to HCWs' needs might account for low adherence rates (Sachs, 2006). Previous research has demonstrated that HCWs repeatedly encounter problems with the usability of the guidelines, which could be detrimental to their uptake in clinical practice (Van Gemert-Pijnen et al., 2005).

The problem with infection control guidelines as a communication means is that they are expert-driven. Expert-driven guideline communication can be characterized by a strong focus on scientific validation, regulation, and legislation (The AGREE Collaboration, 2003). In the design process, higher priority is given to a consensus on content-related issues among experts than to HCWs' practical information needs. This can make the document difficult for individual HCWs to use as a resource and to identify procedures for daily work practice (The AGREE Collaboration, 2003; Van Gemert-Pijnen et al., 2005). In other words, the guidelines' "regulation function" prevails, while the "communication function" often plays a subordinate part. HCWs' tacit knowledge, which is context-dependent and made up of the practical and experiential wisdom of individual HCWs, is rarely taken into account in the development of guideline communication. As a result, infection control guidelines as a communication means are insufficiently targeted to HCWs' norms and values, are hard to use, and eventually evoke reluctance and disregard on the part of the HCWs (Saillour-Glenisson \& Michel, 2003; Shekelle et al., 2000).

A possible solution to enhance the usability of the expert-driven guideline format is to communicate the traditional, paper-based guidelines in a format that better fits the practical information needs of the HCWs. Presenting guidelines on a website facilitates the inclusion of hyperlinks to additional resources and multimodal functionalities, such as instructional videos. Consequently, in-depth information can be available without affecting the guidelines' readability (Fervers et al., 2005). The 
development of a website allows the direct involvement of HCWs, which can lead to a higher level of usability. In a user-centered design process, HCWs will be invited to make their tacit knowledge concerning infection control explicit, stimulated to make their own decisions about directions and strategies for improvement, and are led in those actions (McCoy et al., 2001; Murphy, 2002; Van Gemert-Pijnen et al., 2003). Considering usability prior to development of a first prototype of a website may prevent uncovering pitfalls in the system after its implementation, which can be costly and avoid reluctance among intended users (Thomas and Bevan, 1996).

Various research methods are available to develop communication means with a high level of usability. These methods can be divided into usability testing and usability inspection (Holzinger, 2005; Hom, 1998; Jaspers, 2009; Nielsen, 1993). In usability testing, representative users work on typical tasks using the system (or the prototype) and the evaluators use the results to see how the user interface supports the users to do their tasks. The most common employed methods are thinking aloud, field observation, and questionnaires (Holzinger, 2005). In usability inspection, usability specialists and sometimes software developers or other professionals, examine usability-related aspects of a product or system. Various inspection methods are available, such as heuristic evaluation, cognitive walkthroughs, and action analysis (Holzinger, 2005). Since it is often thought that experts are not able to identify real user problems (De Jong \& Lentz, 2006), and problems with the quality of guideline communication are particularly caused by their expert-driven character, it is better to perform usability testing when improving the format of infection control guidelines. Of all usability testing methods, thinking aloud has been most often used in the health care domain (Jaspers, 2009). Thinking aloud involves having an end user continuously verbalizing thoughts while using a system, which provide insight into the underlying causes for usability problems and requirements for improvement (Holzinger, 2005; Jaspers, 2009; Nielsen, 1993).

In this chapter, we will first describe a study that diagnosed the usability problems HCWs encountered while using traditional, paper-based infection control guideline communication. Next, we will describe three general design principles for the communication of infection control guidelines based upon the findings of the first study. Then we will describe how, according to these principles, the content of the paper document was converted into a website. Subsequently, we will present the results of the second study, in which HCWs worked with a test version of the website. This second study also served as a formative evaluation of the website before it was launched online, with the purpose of "test running" various aspects of the website and to verify whether the design team did not miss any errors. The usability issues raised by this study were solved prior to the website's launch. Finally, we will compare the two communication modes in terms of efficiency, effectiveness, and satisfaction rates. Ultimately, our study resulted in the creation of a website that facilitates HCWs with usable infection control guidelines that enhance the decision making process to deliver safe health care. 


\subsection{Methods}

\subsubsection{Research context}

Due to increased international trade in health services, Methicillin Resistant Staphylococcus aureus (MRSA) is one of the organisms that most commonly contributes to the rapid spread of health care-associated infections (Pittet \& Donaldson, 2006). The Dutch-German research project EUREGIO MRSA-net Twente/ Münsterland addresses this problem at a cross-national level, and the study reported here was conducted as part of this project (Friedrich et al., 2008). Therefore, MRSA guidelines served as a case for study. The study was performed in one Dutch and one German hospital in the border region, with capacities of 1070 and 1500 beds, respectively. The first study was performed in June 2006; the second took place in July 2007. The two studies reported in this chapter were part of a user-centered design process aimed at the gradual development, implementation, and evaluation of a website with MRSA guidelines (Kinzie et al., 2002). The studies reported here were part of the development phase, next to a content analysis of existing guidelines (see Chapter 2 ), a Card Sort Study $(n=10)$ that generated the website's menu structure, and prototyping interviews $(n=14)$ which led to the website's final lay-out (see Chapter 3 ). After the website's launch, we identified factors affecting successful adoption of the website among HCWs (see Chapter 6) and the position of the website among other online sources about MRSA (see Chapter 5 ). In each phase of this user-centered design process, the sample included physicians, nurses, and nursing assistants.

\subsubsection{Research design}

In the initial study, 28 HCWs (4 nursing assistants, 16 nurses, and 8 physicians) were asked to complete MRSA-related tasks using the existing paper-based MRSA protocol enforced in their hospital at the time of the research, while thinking aloud. The protocol consisted of a 50 pages, one-sided printed document, including a table of contents that comprised the title and page numbers of the various chapters. The protocol did not include any additional navigation aids, such as an index. The findings from this initial study gave us insight into usability problems HCWs faced when using a paper-based document. Based on the results, three general design principles were formulated. We revised the presentation and structure of the document according to these principles, which led to a test version of the website. The website's functionalities and navigational structure can be found in Table 4.2. In the second study, the same 28 HCWs completed the identical set of tasks as in the first study while thinking aloud again, but now using the test version of the website. This second study aimed to verify whether the website overcame the problems observed in the initial study and to detect any missed errors before the website was implemented online.

The participants were asked to think aloud while performing the tasks. Thinking aloud is a widely used method for testing the usability of software, interfaces, websites, and (instructional) documents. The basic principle of this method is that potential users 
are asked to complete a set of realistic tasks with the tool tested, and to constantly verbalize their thoughts while working on the tasks. The method has high face validity since the data obtained reflect the actual use of the tool and not the participants' judgements about its usability (Van den Haak, 2008).

In our study, the tasks consisted of what if-tasks. A total of 27 different tasks were formulated, together representing all categories of the MRSA guidelines by the Society for Health care Epidemiology of America (SHEA) (Muto et al., 2003), which include surveillance cultures, hand washing procedures, contact isolation procedures, minimizing cross-contamination procedures (isolating, cleaning), and eradication (antibiotic treatment). A few task examples are:

- You are attending to an MRSA patient in an isolation room. Using the guidelines, can you say aloud which preventive measures you must take in order not to transmit MRSA to other patients or personnel? (SHEA category: contact isolation procedures);

- You have been colonized with MRSA yourself and the results of the first screening cultures are negative. Using the guidelines, can you say aloud whether you can restart your working activities again? (SHEA category: surveillance cultures);

- You want to treat an MRSA patient against MRSA colonization. Using the guidelines, can you say aloud what you need to do? (SHEA category: antibiotic treatment).

The tasks were validated by one Dutch and one German medical microbiologist, who also defined the correct task outcomes. A task was considered to be completed successfully if a HCW found the right solution to the task independently, without help from the test administrator. Van Gemert-Pijnen (2003) already demonstrated this method to be valid. Each participant carried out at least one task from each SHEA category. The order of the tasks was permutated among respondents in order to both ensure that each task was performed an equal amount of times among all respondents, and to reduce possible learning effects caused by respondent's memory about the guideline content gained during performance of earlier tasks.

We used the concurrent thinking-aloud technique proposed by Ericsson and Simon (1993), which implies that the test administrator gives non-directive reminders to continue verbalization after 15 to 20 seconds of silence. Some doubts have been raised about validity and reliability of the thinking- aloud technique, but this critique focused on thinking aloud as a technique to study cognitive processes rather than to identify usability problems (Boren and Ramey, 2000). A more relevant question is the influence of thinking aloud on task performance time. Concurrent thinking aloud tends to increase the time required to arrive at a solution compared with silent controls, presumably because of the extra time required to verbalize. However, concurrent thinking aloud does not affect cognitive processing. Extensive research, reviewed by Ericsson and Simon (1993), indicated that direct concurrent thinking-aloud does imply some slowing of performance, but probably has no reactive effect. Moreover, it is likely that the delay in task performance caused by thinking-aloud is not variable within subjects, and since we applied a within-subjects design (the respondents were exposed to both conditions), the issue of the influence of response time on task 
performance becomes less significant.

The study was conducted in a silent, isolated room in the hospital ward where the participant was employed and lasted strictly one hour. No time limit was imposed on the respondent to work on a task, implying that respondents performed as much tasks as they could during one hour. The tests were audiotaped with prior consent of the respondents and were transcribed verbatim afterwards.

\subsubsection{Study population}

We included 14 Dutch and 14 German respondents in our sample, yielding a total sample size of 28. Respondents were recruited on a voluntary basis. Eligible HCWs were at least 18 years old and Dutch- or German-speaking. They did not need to have prior knowledge or experience with the use of either paper- or web-based infection control guidelines. 15 of the respondents were male, 13 were female, and altogether had a mean age of 38.2 years (range $24-57)$. The majority $(n=16 ; 57.1 \%)$ used the Internet more than twice a month to retrieve work-related information.

Every respondent in this sample participated in the study with the paper-based guidelines as well as in the study with the website. In other words, the exact same set of individuals was involved in the first study as in the second, so the results could be validly compared. Because of the long time between the two studies (over one year), a test-retest effect is unlikely.

\subsubsection{Outcome measures}

The main standard that provides guidance on usability is ISO 9241, which describes usability as "the extent to which a product can be used by specified users to achieve specified goals with effectiveness, efficiency, and satisfaction in a specified context of use" (International Organization for Standardization, 1998).

In a review of 180 studies on usability, Hornbæk (2006) provides an overview of existing measures employed to measure usability. He classified the measures into the three groups effectiveness, efficiency, and satisfaction of the ISO 9241 standard. The typical measures to evaluate the concepts are:

- Effectiveness: task completion, accuracy, recall, completeness, and quality of outcome;

- Efficiency: time, input rate, mental effort, usage patterns, communication effort, and learning measures;

- Satisfaction; preference, satisfaction with the interface, and attitudes and perceptions

Several of these measures were included in our study (see Table 4.1). These indicators can be applied to measure the usability of nearly every document or object. Actually, usability testing has frequently been employed to investigate the usability of paperbased instructional communication (e.g., Gould and Lewis, 1985; Loorbach et al., 
Table 4.1. Usability components and outcome measures

\begin{tabular}{|c|c|c|c|}
\hline Usability component & Subconstruct & Outcome measures* & Data source \\
\hline \multirow[t]{6}{*}{ Effectiveness } & Task completion & Successfully completed or not & Observation \\
\hline & Information structure problems & $\begin{array}{l}\text { Number of encountered problems due to navigation } \\
\text { structure or arrangement of information }\end{array}$ & Verbalization \\
\hline & Matching problems & $\begin{array}{l}\text { Number of encountered problems due to mismatching } \\
\text { between user's and expert's vocabulary }\end{array}$ & Verbalization \\
\hline & $\begin{array}{l}\text { Information quality problems: } \\
\text { Incompleteness }\end{array}$ & $\begin{array}{l}\text { Number of encountered problems due to incomplete } \\
\text { information }\end{array}$ & Verbalization \\
\hline & $\begin{array}{l}\text { Information quality problems: } \\
\text { Incomprehensibility }\end{array}$ & $\begin{array}{l}\text { Number of encountered problems due to incomprehen- } \\
\text { sible information }\end{array}$ & Verbalization \\
\hline & $\begin{array}{l}\text { Information quality problems: } \\
\text { Inaccuracy }\end{array}$ & $\begin{array}{l}\text { Number of encountered problems due to inaccurate } \\
\text { information }\end{array}$ & Verbalization \\
\hline \multirow[t]{4}{*}{ Efficiency } & Time & Number of seconds needed to complete scenario & Observation \\
\hline & Effort & Number of actions needed to complete scenario & Observation \\
\hline & Search strategy: Orienteering & Frequency with which this strategy is applied & Observation \\
\hline & Search strategy: Teleporting & Frequency with which this strategy is applied & Observation \\
\hline \multirow[t]{3}{*}{ Satisfaction } & Design & Number of expressions related to design & Verbalization \\
\hline & Content & Number of expressions related to content & Verbalization \\
\hline & Features & Number of expressions related to features & Verbalization \\
\hline
\end{tabular}

Both expressed in number of scenarios during which problems were faced as well as in number of respondents who encountered the problem. Repeated problems were only counted once per scenario. 
2007). This justifies the application of the identical usability measures to both the paper document (study 1 ) and the website (study 2).

Effectiveness is defined as the accuracy and completeness with which users achieve certain goals (Frøkjær et al., 2000). According to Frøkjær et al. (2000), indicators of effectiveness include task completion and information problems. We used task completion as the primary, objective indicator of effectiveness by scoring for each scenario whether or not it was completed successfully. These judgments were verified by the two medical microbiologists who validated the scenarios (see section 4.2.2.). A second measure of effectiveness was the number of problems related to information quality that arose while HCWs worked with the guidelines. Information quality problems can be related to incompleteness, incomprehensibility, or inaccuracy (Bennett et al., 2004; Van Gemert-Pijnen et al., 2005). During analysis, two other types of problems emerged: inadequate information structure and matching problems. We identified information problems by closely studying verbal indicators or problems experienced by HCWs (Van den Haak, 2008).

Efficiency is defined as the time and effort with which users achieve certain goals (Frøkjær et al., 2000). We measured the time aspect by registering the number of seconds HCWs spent navigating to and interacting with the content. Effort was measured by recording the number of actions it took a respondent to complete a task. In the first study, where HCWs worked with paper documents, an action was defined as a page-turn or starting to read a new section on the same page out loud. In the second study, an action was defined as a mouse-click (on a hyperlink) or entering a search term in the search engine. Since respondents used a different communication mode in each study, the number of actions could not be compared validly; turning 40 pages in a paper-based document demands a higher cognitive load than clicking on a hyperlink. However, we did count the number of actions in both communication modes in order to have a rough indication of their extraneous cognitive load (Chandler and Sweller, 1991). Cognitive load theory claims that the attention and working memory of an individual are limited. The extraneous cognitive load, which concerns cognitive demands during learning and do not foster the actual objectives of the learning material, is generated by the manner in which information is presented to learners and is under the control of the designers (Holzinger et al., 2009; Pollock et al., 2002). Extraneous cognitive load can be attributed to the design of the instructional materials, such as cross-references or navigation elements.

As an additional measure of efficiency, we examined whether HCWs employed different information retrieval strategies when faced either with a paper document or a website. We registered every action respondents undertook during scenario performance. This generated observational data used to analyze HCWs' information retrieval strategies. We identified two main search strategies: orienteering and teleporting. Orienteering involves reviewing both prior and contextual information to narrow in on the actual information target, often in a series of steps, without specifying the entirety of the information needed up front. Teleporting concerns jumping directly to the information target (Teevan et al., 2004). Teleporting is a more efficient strategy, as the user does not have to react to stimuli from the medium, 
which takes more time and effort. We coded a respondent's search strategy as orienteering if it took more than one action to retrieve the relevant information, and we assumed respondents teleported when they needed only one action to achieve their goal.

Satisfaction includes the users' comfort with and positive attitudes towards the use of the communication means. We assessed satisfaction by analyzing both respondents' verbal utterances during scenario performance (think-aloud data), next to their overall opinion concerning the communication means' usability (which we asked for after they finished the tasks). Expressions were related to design, content, and features of the communication means. Since we were primarily interested in exploring HCWs' first reactions to the website rather than testing a number of propositions, we did not use a standardized questionnaire to determine user satisfaction, such as the WebMedQual scale (Provost et al., 2006; Van Velsen et al., 2008).

\subsubsection{Analysis}

The respondents had to solve identical tasks in the first and second study. The number of completed scenarios, however, differed between the two studies; the respondents appeared to finish more scenarios in the second study. As the scenarios differed considerably in their nature (see section 4.2.2.), only the scenarios that were performed by a respondent both in the first and the second study were involved in the analysis.

Data from one randomly selected transcribed thinking-aloud test were analyzed independently by two researchers (FV, JvG) using deductive analysis, implying that the coding categories were derived from the conceptual framework presented in Table 4.1. (Patton, 1990). Half of the coding categories involved observations made by the researcher, whereas the other half concerned respondents' verbalizations. Repeated or reworded descriptions of the same problem were only counted once per task. Disagreement was solved by discussion after jointly reviewing the fragment. Subsequently, all transcribed data were coded by the principal investigator (FV). Overall, 1381 fragments of verbalization concerning effectiveness, efficiency, and satisfaction were identified. Since the data set was too large to have it reviewed independently by two researchers, a random selection of $5 \%$ of all coded fragments was coded by a second independent researcher (JVG). Agreement on categorization of the problems and requirements was satisfactory (Cohen's Kappa $=.78$ ) (Landis and Koch, 1977).

In order to conclude whether the website enabled the users to retrieve the required information more effectively (rate of successfully completed scenarios) and efficiently (the number of seconds and the amount of actions to complete a scenario) compared to the paper document, Paired Samples T Tests were carried out using SPSS 16.0. To detect significant differences between the type of information problems and search strategies that occurred in both conditions, Chi-square-tests were conducted. 


\subsection{Results}

This section is divided into four parts. First, we present a qualitative description of the problems that HCWs encountered while using the paper-based guidelines (in study 1 ), then we describe how these findings were incorporated in the design of a user-oriented guideline website. Subsequently, we describe the problems that were encountered while respondents used the website (in study 2). Finally, we present quantitative data to compare the two communication means.

\subsubsection{Problems with the paper-based guidelines}

More than half of the performed scenarios were not completed successfully. Furthermore, it took the respondents an average of 383 seconds (six minutes) to finish a scenario. Given that in reality, HCWs abandon a search after about two minutes, the effectiveness and efficiency of the paper-based guidelines can be rated as low (Yu et al., 2007). The observations and the think-aloud verbalizations made clear what the main problems were.

\section{Effectiveness problems}

Approximately one third of the usability problems were due to a mismatch between the experts' and the HCWs' vocabulary (see Table 4.4.). For instance, a respondent looked for the word "treatment" in the table of contents, whereas the guideline document used the word "decolonization therapy".

Many other usability problems were caused by the poorly structured information. These problems were mainly faced during the "examine results" phase (Marchionini, 1995). This is the phase during which respondents examined relevant sections of the document to identify whether or not it contained the required information. Respondents expected to find the required information in a particular section or by employing a specific navigation strategy, but the opposite appeared to be true:

"Now I have to read the complete index. An alphabetically ordered-index would make it more practical, so I can immediately and more quickly find what I need."

(Respondent 11, German nurse).

Other examples of information structure problems that were encountered with the paper document, are:

- Difficulties with finding specific pages;

- Lack of list-wise presented information;

- Lack of decision trees;

- Lack of tables;

- Little space between the lines. 
The low effectiveness rate seemed to be mainly caused by insufficient quality of information. Information appeared to be inaccurate (procedures were outdated or different from HCWs' tacit knowledge), incomprehensible (containing professional jargon and unclear procedural information), or incomplete. The latter was the largest hindrance to successful task completion. Respondents indicated that the information was too concise to enable them to make a safe decision for clinical practice, as the following quote illustrates:

"So this guideline only prescribes that I'm not allowed to perform activities involving direct and indirect patient contact. I don't know what indirect contact means. Where it begins and where it ends. Generally speaking, everything that we do involves indirect patient contact. Therefore I don't know whether I'm allowed to work here on the ward, or down in the archive, or... that's not clear to me."

\section{(Respondent 9, German nurse)}

Additionally, respondents wished for more practical information, particularly guidelines related to MRSA-positive HCWs:

"If a patient is colonized with MRSA, the medical microbiologist is responsible. And then the guidelines serve as a manual for what to do. But for MRSA-positive staff, there is nothing."

\section{(Respondent 11, Dutch nurse)}

Overall, the information problems provided us with 165 additional key questions that were not answered by the paper-based guidelines, but their answers are nevertheless required for HCWs to deliver safe health care. To mention a few:

- In what order should I put on and take off personal protective equipment?;

- Which materials should I throw away after discharge of an MRSA-positive patient from an isolation room?;

- An MRSA-positive patient should be transported from one ward to another within the hospital. Which personal protective equipment should I wear when accompanying this patient?

\section{Efficiency problems}

Respondents particularly applied the orienteering strategy when using the guidelines. Every respondent employed this strategy during one or more scenarios, particularly when they encountered difficulties with translating the scenario into a meaningful search question, or had no particular idea about where to find the required information. In such an event, the respondent started to review the different sections of the guidelines (e.g., by means of the table of contents) and checked each section for its possible relevance, which required much of the respondents' time. 
Teleporting, a more efficient search strategy, occurred less frequently (see Table 4.5.). Teleporting was mainly performed when the respondent remembered having read about the topic earlier, while solving a previous scenario. This required a high mental effort from the users, as they had to rely on their memory in order to solve scenarios. Sometimes, both strategies were combined: respondents commonly fell back on the orienteering strategy after an instance of ineffective teleporting.

\section{Satisfaction problems}

Satisfaction refers to the users' comfort with and positive attitudes towards the use of the guideline format. During scenario performance, several respondents verbalized feelings of annoyance or frustration regarding the design:

"I can't find it again. The fact that I've been searching for ten minutes now and still do not know for sure that the answer is right, that is not good!"

\section{(Respondent 7, Dutch physician)}

Other important verbalizations concerned dissatisfaction with the absence of information presented in list form $(n=4)$, important information not printed in bold $(n=3)$, and the absence of tables $(n=7)$ and tree diagrams $(n=5)$. One respondent suggested the solution of providing several search options that consider the different search strategies that people use:

"A table of contents and an index... Like in a book. Most people are acquainted with that, so an index should be in the back. And a table of contents in the front. And then each chapter should be considered a separate category."

\section{(Respondent 10, Dutch nurse)}

Furthermore, respondents believed that the guidelines should more explicitly communicate what one should or should not do in a particular situation $(n=15)$. Guidelines should be "clear and concise" $(n=14)$ and "contain stone-hard facts".

\subsubsection{Designing the website}

Our belief that a web-based format would improve the usability of the guidelines (see section 4.1.), was strengthened by the findings of study 1. Although information quality problems (inaccuracy, incomprehensibility, and incompleteness) could have easily been prevented by solely improving the information and maintaining the paperbased format, the (1) mismatch and (2) "information structure" problems could be more optimally addressed via a website. (1) A content management system would enable the design team to add and delete keywords to the search engine's database that aid in matching system's with HCWs' vocabulary, and (2) a web-based format would allow the inclusion of combining several search options (search engine, categorical search, frequently asked questions) and could aid to enhance the clarity of the information structure, like a breadcrumb trail. 
Table 4.2. Design principles for a website with infection control guidelines based on a study with paper-based guidelines $(\mathrm{N}=28)$

\begin{tabular}{|c|c|c|}
\hline Usability component & Design principle & System description \\
\hline Effectiveness & $\begin{array}{l}\text { Add practical, action oriented con- } \\
\text { tent (in order to avoid information } \\
\text { quality problems), communicated } \\
\text { in HCWs' vocabulary (to prevent } \\
\text { matching problems) }\end{array}$ & $\begin{array}{l}\text { The } 165 \text { additional key questions that emerged from the first study } \\
\text { served as input for the content of the website. Answers were based on } \\
\text { national MRSA guidelines (Dutch Working Party on Infection Prevention, } \\
2005 \text {; The Committee for Hospital Hygiene and Infection Prevention at } \\
\text { the Robert Koch Institute, 1999), and were validated, complemented, and } \\
\text { actualized by the Dutch and German medical microbiologists involved in } \\
\text { the research project. } \\
\text { In order to communicate the guidelines' content to dovetail with HCWs' } \\
\text { vocabulary, the website used words such as "get rid of MRSA" instead } \\
\text { of "eradication therapy", "take swabs" instead of "perform screening } \\
\text { cultures", "outbreak" rather than "epidemic situation", etc. A dedicated } \\
\text { content management system enabled the project team to add and delete } \\
\text { keywords to the search engine's database that aid in matching system } \\
\text { with HCWs' vocabulary. } \\
\text { See Figure } 7 \text { in the middle of this thesis for an example. }\end{array}$ \\
\hline Effectiveness & $\begin{array}{l}\text { Present guidelines in a multimodal } \\
\text { way (in order to overcome informa- } \\
\text { tion structure problems) }\end{array}$ & $\begin{array}{l}\text { Each of the } 165 \text { additional key questions was answered according to a } \\
\text { standardized format based on usability guidelines and a content analysis } \\
\text { of existing guidelines to serve both a regulation and communication } \\
\text { function, with important items placed consistently at the top center. } \\
\text { Scroll stoppers were avoided as much as possible and moderate white } \\
\text { spaces were used (Koyani et al., 2006). Each answer comprised a title, } \\
\text { a short answer, an instructional video, comments, and (scientific) refer- } \\
\text { ences such as newspaper articles, links to other websites for further } \\
\text { reading, and multi-media examples. This structure was based on a } \\
\text { content analysis of existing paper-based guidelines (see Chapter 2). } \\
\text { In order to keep information accurate, revision dates and the latest news } \\
\text { were included. Furthermore, visual aids such as tables and tree diagrams } \\
\text { were added. } \\
\text { See Figure } 8 \text { in the middle of this thesis for an example. }\end{array}$ \\
\hline Efficiency & $\begin{array}{l}\text { Consider different search strategies } \\
\text { HCWs employ by incorporating three } \\
\text { search options }\end{array}$ & $\begin{array}{l}\text { In order to make the search process more efficient, the system should } \\
\text { allow the user to rapidly switch from one search strategy to another } \\
\text { and users should always be able to keep track of their location within } \\
\text { the system. Therefore, answers to questions were retrievable through a } \\
\text { search engine, a menu structure with categories, and frequently asked } \\
\text { questions. We also incorporated a breadcrumb trail. } \\
\text { The incorporation of three search options and a breadcrumb trail enable } \\
\text { HCWs to teleport rather than orienteer and therefore find the relevant } \\
\text { information more rapidly with less effort (i.e., more efficient). } \\
\text { See Figure } 6 \text { in the middle of this thesis for an example. }\end{array}$ \\
\hline
\end{tabular}


We formulated three general principles for the improved communication of infection control guidelines, based on our understanding of HCWs' problems with the paperbased guidelines that resulted from the first study. These principles are presented in Table 4.2., each with some examples of how they were applied to the website. An extensive system description has been published elsewhere, including a description of the user-driven design process (see Chapter 3; Verhoeven et al., 2008). Two design principles involve effectiveness and one concerns efficiency. We assume that enhancement of effectiveness and efficiency will indirectly imply a higher user satisfaction. We would like to emphasize that we did not change the content of the guidelines. Rather, we rephrased them in a more instructional style, used words that were more familiar to the HCWs, presented the guidelines multimodally, and presented them in a structure that enables a more efficient search strategy. Two medical microbiologists involved in the research project verified the clinical content of the guidelines before any respondent was exposed to the website.

\subsubsection{Problems with the website}

Nearly $90 \%$ of performed tasks were completed successfully. It took the respondents an average of 132 seconds to finish a task.

\section{Effectiveness problems}

Although the number of encountered matching-, completeness-, and accuracyrelated problems dropped considerably when HCWs worked with the website (see section 4.3.4.), an equal number of usability problems occurred during both studies. This seemed to be due to an increase in problems related to information structure. Whereas HCWs in the first study mainly faced information structure problems during the "examine results" phase, they now experienced information structure problems mainly throughout the "reflect, iterate, stop" phase. In this stage, HCWs already supposed to have detected the relevant information, but experienced problems when reading the answer to the question in more detail. The most salient problem was that respondents did not understand how to navigate within and away from the answer section:

"The answer says 'screening of high-risk patients' and then which sites you have to sample. Oh, I've seen that earlier, hmm... Can I go back?"

(Respondent 3, Dutch nurse).

Furthermore, respondents expected more hyperlinks to additional sources: 
"Hospitalized patients should be treated in isolation. Yes, that is what I was looking for. Notification obligation. I'll click on this word. Can I click here? Oh, I can't click."

(Respondent 3, German nursing assistant)

Other examples of information structure problems encountered with the website, are:

- Search options being unnoticed;

- Information not separated for different occupational groups;

- Lack of a link to a Portable Document Format version of the page;

- Lack of a print button.

\section{Efficiency problems}

Respondents used the teleporting strategy more often than the orienteering strategy when working with the website. The lower frequency of teleporting with the use of the paper-based guidelines might be due to the difficulty of creating a clear mental model of its structure (users are not able to view all pages simultaneously), whereas the transparent interface of the website permitted the respondents to continuously keep track of their position within the system. The various search options, including the table of contents in the category section, were continuously visible to the user. This possibly enabled the user to create a clear mental model of the system structure.

\section{Satisfaction problems}

HCWs uttered several expressions of satisfaction with the website. Several HCWs seemed to appreciate the practical-oriented content:

"I've tried several times to find clear guidelines on our Intranet, but it always contains lengthy declarations about nearly everything and it is almost impossible to comb through. But here, on this website - for instance with the cutlery and the clinical waste - it is described clearly: How one should handle it, and that's it. Not so long-winded. So you know immediately what to do."

(Respondent 14, German physician)

Furthermore, the website's efficiency was perceived as very positive:

"Well, this website works out much better than the paper protocol. That was a whole show of page-turning. I would say this is a major improvement."

(Respondent 7, Dutch physician)

However, the task completion time in the website condition was still not appreciated by all respondents. One respondent remarked, after having completed the scenario 
successfully within 33 seconds, "Wow, that was very hard to find." (Respondent 14, Dutch, physician). This might be due to the great expectations respondents hold regarding the speed with which Internet-based tools function.

\section{Problem solving}

Study 2 also was intended to "test run" various aspects of the website and to verify whether the design team did not miss any errors. Missed errors appeared to be the lack of including hyperlinks when relevant, the difficulties that respondents encountered with navigating in and away from answer pages (see section 4.3.1.), and the website's slow speed, as perceived by several respondents (see section 4.3.3.). These issues were solved before the website was officially implemented online: more hyperlinks were incorporated, an instructional text regarding website use and the website's objective was made accessible via an "About this website" button, and we limited the log file size growth in order to speed up the web pages' load time.

\subsubsection{Comparison of the paper-based guidelines with the website}

\section{Comparison of the effectiveness outcomes}

The website enabled HCWs to complete significantly more scenarios successfully compared to the paper-based guidelines ( $p<.001$ ) as shown in Table 4.3.

The number of usability problems did not substantially decrease when HCWs used the website ( 270 versus 242 problems, as seen in Table 4.4.). The only significant difference concerned matching problems ( $p<.05)$. However, the type of problems encountered by the HCWs differed considerably.

The increased effectiveness of the website seemed to be caused mainly by the inclusion of practical, action oriented content communicated in HCWs' vocabulary, and by incorporating different search options, as the number of matching and information quality problems dropped in the website environment. Remarkably, the number of problems related to information structure doubled when HCWs worked with the website, although this difference was not significant. For instance, respondents did not know how to navigate within and away from the answer on the website. Because the website enabled users to achieve the learning performance within a smaller time frame, more time was available for exploring the underlying information, implying more recognition of problems with the presentation of information (Holzinger et al., 2009).

\section{Comparison of the efficiency outcomes}

Efficiency is only a relevant quality criterion if the communication is effective (saving time to find an incorrect solution does not make any sense). Therefore, we computed the task completion time for successful completed scenarios only. The mean completion time per scenario was 383 seconds with the paper-based guidelines versus 132 seconds in the web-based condition. The website thus allowed the HCWs 
Table 4.3. Overview of success of completed scenarios with paper-based guidelines in study 1 and the website in study 2 $(\mathrm{N}=28)$

\begin{tabular}{|c|c|c|c|c|}
\hline & \multicolumn{2}{|c|}{ Paper-based guidelines } & \multicolumn{2}{|c|}{ Website } \\
\hline & $\mathrm{N}$ & $\%$ & $\mathrm{~N}$ & $\%$ \\
\hline Completed successfully & 67 & 47.5 & $124^{*}$ & 87.9 \\
\hline Not successfully completed & 74 & 52.5 & 17 & 12.1 \\
\hline Total & 141 & 100 & 141 & 100 \\
\hline
\end{tabular}

Note: The Paired Samples T-test was used to detect significant differences between the two conditions. Time between studies was 13 months.

* $p<.001$

Table 4.4. Overview of information problems encountered when using paper-based guidelines in study 1 and the website in study $2(\mathrm{~N}=28)$

\begin{tabular}{|c|c|c|c|c|c|c|c|c|}
\hline \multirow{3}{*}{ Problem type } & \multicolumn{4}{|c|}{ Paper-based guidelines } & \multicolumn{4}{|c|}{ Website } \\
\hline & \multicolumn{2}{|c|}{$\begin{array}{l}\text { Scenarios } \\
(\mathrm{N}=141)\end{array}$} & \multicolumn{2}{|c|}{$\begin{array}{l}\text { Respondents } \\
(\mathrm{N}=28)\end{array}$} & \multicolumn{2}{|c|}{$\begin{array}{l}\text { Scenarios } \\
(\mathrm{N}=141)\end{array}$} & \multicolumn{2}{|c|}{$\begin{array}{l}\text { Respondents } \\
(\mathrm{N}=28)\end{array}$} \\
\hline & $\%$ & $\mathrm{n}$ & $\%$ & $\mathrm{n}$ & $\%$ & $\mathrm{n}$ & $\%$ & $\mathrm{n}$ \\
\hline Mismatch between HCWs' and experts' vocabulary & 53.9 & $76^{*}$ & 100.0 & 28 & 34.8 & 49 & 82.1 & 23 \\
\hline Information structure & 29.1 & 41 & 92.9 & 26 & 48.9 & 69 & 71.4 & 20 \\
\hline Incompleteness of information & 60.3 & 85 & 96.4 & 27 & 44.0 & 62 & 82.1 & 23 \\
\hline Inaccuracy of information & 24.1 & 34 & 75.0 & 21 & 14.9 & 21 & $39 \cdot 3$ & 11 \\
\hline Incomprehensibility of information & 24.1 & 34 & 71.4 & 20 & 29.1 & 41 & 75.0 & 21 \\
\hline Total & & 270 & & & & 242 & & \\
\hline
\end{tabular}

Note: Chi-square was computed to detect significant differences between the two conditions. Time between studies was 13 months.

${ }^{*} p<.05$

Table 4.5. Overview of search strategies used with paper-based guidelines in study 1 and the website in study $2(\mathrm{~N}=28)$

\begin{tabular}{|c|c|c|c|c|c|c|c|c|}
\hline \multirow{3}{*}{ Strategy } & \multicolumn{4}{|c|}{ Paper-based guidelines } & \multicolumn{4}{|c|}{ Website } \\
\hline & \multicolumn{2}{|c|}{$\begin{array}{l}\text { Scenarios } \\
(N=141)\end{array}$} & \multicolumn{2}{|c|}{$\begin{array}{l}\text { Respondents } \\
(\mathrm{N}=28)\end{array}$} & \multicolumn{2}{|c|}{$\begin{array}{l}\text { Scenarios } \\
(\mathrm{N}=141)\end{array}$} & \multicolumn{2}{|c|}{$\begin{array}{l}\text { Respondents } \\
(\mathrm{N}=28)\end{array}$} \\
\hline & $\%$ & $n$ & $\%$ & $n$ & $\%$ & $\mathrm{n}$ & $\%$ & $n$ \\
\hline Only Orienteering & 59.6 & $84^{*}$ & 96.4 & 27 & 34.0 & 48 & 78.6 & 22 \\
\hline Only Teleporting & 31.2 & 44 & 75.0 & 21 & 52.5 & $74^{*}$ & 78.6 & 22 \\
\hline Combination & 9.2 & 13 & 17.9 & 5 & 13.5 & 19 & 50.0 & 14 \\
\hline
\end{tabular}

Note: Chi-square was computed to detect significant differences between the two conditions. Time between studies was 13 months.

* $\mathrm{p}<.05$ 
to complete a scenario over four minutes faster (251 seconds) than the paperbased guidelines ( $p<.001)$. The action criterion, however, led to different results. The respondents had to perform more actions in order to complete a scenario in the webbased environment than in the paper-based environment: 3.9 versus 3.0 actions (not significant). Table 4.5. shows the search strategies HCWs employed when using each search strategy.

The website allowed the HCWs to use the teleporting strategy significantly more frequent compared to paper-based guidelines (p<.05). As can be seen in Table 4.5., the less efficient, time-consuming orienteering strategy was employed significant less frequently with the website compared to paper-based guidelines ( $p<.05)$.

\section{Comparison of the satisfaction outcomes}

Overall, the design, content, and features of the website were perceived better than those of the paper-based guidelines. The respondents were very satisfied with several aspects of the website. While thinking aloud they verbalized their appreciation of the fact that the information was "conveniently arranged" $(n=13)$, and had an "intelligible design" $(n=7)$.

Respondents were also positive about the practical content of the website. Five HCWs labeled the content as "very informative" and five expressed that the website would be a valuable addition to existing information sources about MRSA:

“I recently looked up 'MRSA' in 'Herold', a book about medicine, and it only contained one sentence about MRSA. But it is also the book people use in medical school. That one sentence is really insufficient. The book only says USA 40\% MRSA, the Netherlands $1 \%$. In comparison, I think this website is very good."

\section{(Respondent 1 German physician)}

The same respondent even mentioned that the practical content of the website should "become a standard in the education of each health care worker". The overall usability was perceived as good by the HCWs. Eight of them explicitly stated that the website was "easy to work with" and six people valued the combination of three different search options.

\subsection{Discussion}

The objective of our research was to tailor the communication of paper-based infection control guidelines to a communication format that better fits HCWs' practical information needs. Our first study revealed that the existing guidelines hinder their application by HCWs in practice because of usability problems related to poorly structured information, insufficient information quality, and a mismatch between experts' and HCWs' vocabulary. Based on HCWs' information needs, we applied three main design principles of usability to a website: better navigation (the guidelines 
should be searchable in several ways), multimodality (the guidelines should not be presented as text only), and action orientation (the guidelines should be presented as HCWs' behaviors). The most obvious outcomes of our study were the outperformance of the website with respect to effectiveness (the number of correctly completed scenarios), efficiency (the time needed to complete these scenarios), and a higher degree of satisfaction with the website compared to the paper-based guidelines.

The increased effectiveness and efficiency of the website compared to the paperbased format seemed to be caused mainly by the inclusion of practical, actionoriented content communicated in HCWs' vocabulary, and by incorporating multimedia examples and different search options, as can be concluded from the decreased number of matching and information quality problems in the website condition. Remarkably, however, the number of problems related to information structure doubled when HCWs worked with the website. Further inspection of the observation and verbalization data suggested that the respondents spent less time navigating to the answer in the web-based environment, but once the relevant information was detected, they needed more time for navigating within and away from the answer compared to the paper-based guidelines. This might be ascribed to the fact that the navigation structure (the route to the answer) was based on research involving real users (Card Sort, see Chapter 3), in contrast to the standardized answer format which was not presented to users during the design phase, but was based purely on a content analysis of existing guidelines (see Chapter 2 ) and usability design principles (see Chapter 3).

Other usability issues that were raised during the website evaluation were the slow speed of the pages load times (see section 3.3.3.), the lack of hyperlinks, and the difficulty of moving in and away from web pages (see section 3.3.1.). Also, the number of incomprehensibility problems did not decrease, as we expected. One might question why these issues were not already addressed during website design. A possible explanation is that the design team mainly concentrated on preventing the problems detected by the thinking-aloud tests in study 1 . Possibly, the design team's attention for choices that might have easily prevented errors (such as an easily accessible glossary for difficult terms), was thereby distracted.

Overall, our results show that a website is a suitable mode for communicating infection control guidelines. Compared to paper-based guidelines, the website functions better with respect to all three usability components: effectiveness, efficiency, and satisfaction.

\subsubsection{Implications for further research}

Our study confirms two major advantages of user-driven design. First, user-driven design yields more efficient and effective communication means. Second, users develop a sense of ownership for the medium (website), so it can be integrated into the environment more quickly (Abras et al., 2004). Each of these benefits provides implications for further research. 


\section{More efficient and effective communication means}

Our study demonstrated that user-driven design contributes to the website's efficiency and effectiveness. However, considering the website's effectiveness, it appeared that although more scenarios were completed successfully with the website than with the paper-based guidelines, the number of usability problems did not substantially decrease. A shift could be observed in the type of problems that were encountered: respondents experienced twice as many problems related to information structure when working with the website compared to the paper document. However, the majority of these problems were encountered when the required information had already been retrieved and not so much while HCWs were searching for the answer, as was the case with the paper-based document. We ascribed this to the fact that the navigation menu was based on user-driven design methods (Card Sort, see Chapter 3 ), in contrast to the standardized answer format which was based on usability design principles and a content analysis of existing guidelines (see Chapter 2) with no real users involved. As HCWs are used to guideline documents concentrating on legislation and regulation, they seem to have problems with a more vivid, multimodal presentation of the information and to navigate within and away from the information. More research is required to determine the optimal answer structure that dovetails with HCWs' needs and expectations.

Because HCWs had to perform more and different actions (e.g., link-clicking versus page-turning) in order to complete a scenario with the website than with the paper document (3.9 versus 3.0 actions), it could be expected that the extraneous cognitive load would also increase, leading to longer performance times. However, the website enabled HCWs to complete scenarios over four minutes faster than the paper document. So the extraneous cognitive load was not so much linked to the objective number of actions HCWs undertook, but to medium-specific characteristics: webbased communication supported simultaneous use of different information units via hyperlinks and also conferred the ability to skip unnecessary information. Because HCWs indicated that using the website was much more efficient than the paper-based document, and considering the shorter performance times, it may be concluded that HCWs did not experience a higher cognitive load when using the website, despite the higher number of performed actions. This finding corresponds with recent work from Holzinger et al. (2009). They showed that despite interactive and dynamic media being cognitively more demanding than static media, use of dynamic media does not negatively affect learning performance because no differences were detected between usage of static and dynamic media to learn.

The low extraneous cognitive load experienced by HCWs when using the website was possibly caused by our attempts to minimize HCWs' cognitive load. For example, we visualized procedural information (e.g., hand washing, contact isolation, etc.) in videos, tables, and decision trees, and presented action oriented steps in list forms. Previous research also demonstrated that the efficiency of visual media is often preferred above written or verbal information, because the latter unduly loads the learner with unnecessary information (Clark et al., 2006). 
Our study showed that learning with presentation of information in combination with pictures, videos, decision trees, and other visual cues, positively affects elaboration of information (since task performance time significantly decreased in the website condition), which is considered to be most effective for learning. This finding is in line with Mayer's multimedia learning theory (Mayer, 2005). Since an efficient format of instructional materials promotes learning (Clark et al., 2006), it may be interesting to further develop the website's educational functionalities. The HCWs in our sample already suggested that the existing website should become a standard in each HCW's education (see section 4.3.4.). Given the growing importance of the Internet to physician professional development, we anticipate on this by building an e-learning system.

Another functionality to lower HCWs' extraneous cognitive load might be incorporating an interactive dialogue functionality into the website. Since users are often unable to express their need for information in a single, self-contained query within the medical domain (Nijland et al., 2008), the dialogue system will allow users to ask for clarification, make corrections to the system's interpretation of utterances, and ask follow-up questions (comparable to the system developed by Chai et al., 2001).

\section{Creating a sense of ownership for infection control guidelines}

The second advantage of user-centered design is that users develop a sense of ownership for the website, so that the website can be integrated into the work environment more quickly (Abras et al., 2004). We realize that commitment to the website among a sample of $28 \mathrm{HCWs}$ is not enough to establish a sense of ownership among all intended users. However, usually when new technologies come about, they are only adopted by a small group of people initially; later, they spread to other people. Diffusion research suggests that a small group of highly respected peers, approximately $10 \%$ of all end users, influences the adoption decisions of the other $90 \%$ (Rogers, 2003). The HCWs who participated in our study positively valued the website's usability and appreciated the practical relevance of the information (see also Verhoeven et al., 2009). If HCWs advocate the website among their peers, the chances for creating a sense of ownership among other HCWs increase, next to the chances for successful implementation.

Since February 19, 2008 the website can be accessed via www.mrsa-net.nl (in both Dutch and German) and has been visited by more than 110.000 unique visitors (d.d. June 17 2009), and now has an average of 450 unique users daily. Although the website has been received relatively well, its implementation in daily clinical practice can still be increased. Therefore, we investigated how adoption of the tool among HCWs can be stimulated by critical success factors that play a role with the diffusion of technologies in health care, such as opinion leaders, communication, training, etc. (Verhoeven et al., 2009). Adoption of the tool is not only determined by the guideline characteristics of form, compatibility, and trialability, but there are also extraneous variables on both the individual (e.g., attitude, knowledge, job satisfaction) and the environmental level (e.g., communication, training, management values) that must 
be considered when implementing a website such as this one (Green and Kreuter, 2006). Most commonly, these extraneous factors are left out of consideration when implementing innovations in health care (Cain and Mittman, 2002; Kaplan, 1997).

Together, these two benefits advocate the application of user-centered design techniques. In health care, however, the culture is still to train users (HCWs or patients) to adapt to poorly designed documents, rather than to design the document to fit the target group's tacit knowledge (Johnson et al, 2005). Based on our findings, we recommend the involvement of HCWs in the design process of guidelines to externalize their tacit knowledge, which has been advocated before (Gross et al., 2001; McCoy et al, 2001; Murphy, 2002; Van Gemert-Pijnen et al., 2005). However, as far as we know, we were the first to actually take action within this field.

The tendency to focus on expert-oriented criteria is also visible in the field of webdesign. Numerous criteria have been developed to guarantee quality of health-related websites that tend to reflect professional concerns, including accuracy, completeness, readability, disclosures, and references (Provost et al., 2006). By contrast, little is known about the user perspective on health websites, although we know that users and experts generate different criteria for assessing the quality of traditional non-web-based information materials (Coulter et al., 1998). Our study serves as paramount evidence of the discrepancy between expert- and user-oriented criteria and demonstrates the usefulness of integrating users' tacit knowledge into system criteria, ultimately suggesting that user-centered design methods should be employed.

\subsubsection{Implications for infection control practice}

Our results suggest that three design principles are important in order to realize a usable website with infection control guidelines:

1) The addition of key questions (and their answers) that arose during the first study and the use of terminology that matches the vocabulary of the users;

2) Presentation of the information in a multimodal fashion; and

3) Enabling users to apply a combination of search strategies.

However, HCWs' needs regarding communication of infection control guidelines are context-specific and therefore, these design principles are not universal. Experts who are responsible for guideline communication need to involve target users in the development process, for instance by employing thinking-aloud research themselves, to determine the exact needs of their intended users. Furthermore, based on our findings we suggest that when developing infection control guideline communication, experts should consider:

1) How to optimally present the guideline content in such a way that both safety regulations/ legislation ("regulation function") and practical information 
("communication function") are optimally integrated;

2) Develop the most efficient communication format possible in order to lower HCWs' extraneous cognitive load. HCWs will abandon modes of communication demanding a high cognitive load, as workload in the health care setting is already high; and

3) Consider external factors when implementing the new guideline communication means.

\subsubsection{Limitations}

The major limitation of this study, which is general to all task-oriented studies, is that we did not measure HCWs' actual behavior. HCWs may answer questions in this type of studies in a way that they think is socially desirable, or "right", rather than the way in which they actually practice. However, the available evidence supports the validity of task-oriented studies for measuring differences between groups (Carey \& Garett, 1996).

Our study approach concentrated on the ability of HCWs to perform scenarios with infection control guidelines. This task-oriented approach contrasts with the "systemoriented" approach in which the quantity of relevant documents retrieved are focus of attention. Measures like recall and precision are used. Although these measures ignore the user and do not capture the interactive nature of the actual use of systems, they are useful in measuring retrieval system performance (Hersh et al., 2002). Therefore, we also investigated the website's system performance, which has been reported elsewhere (Tigelaar et al., 2008).

Although qualitative methods enabled us to optimally explore the user perspective, we could have benefited from quantitative techniques. The type of usability testing we used is probably the most expensive and time-consuming of the available methods. Numerous other approaches are discussed in the evaluation literature. These include cost-benefit analyses, document analysis and questionnaires (Kaplan, 1997). For instance, several validated questionnaires are available to measure user satisfaction. Perhaps existing validated questionnaires such as the WebMedQual scale would have produced more generalizable results (Provost et al., 2000). However, since the field of user-driven guideline communication is still in its infancy (Elling, 1991; Van GemertPijnen, 2003), we were primarily interested in exploring the way HCWs work with the website we developed rather than revealing what a group of HCWs thinks about the website (Van den Haak, 2008).

The aim of our study was to develop a website that enables HCWs to efficiently and effectively retrieve infection control guidelines. Although the studies reported in this chapter indicate this goal to be achieved, the ultimate aspiration would be to actually improve HCWs' adherence to infection control guidelines. A randomized controlled trial (RCT) would be the best way to evaluate whether implementation of the website 
in daily work practice is truly effective in this regard. However, conducting an RCT to measure the influence of website use on behavior change is a great challenge, for the reason that use of a website is hard to control next to the general drawbacks inherent to RCTS such are their long duration and the high costs (Lachin et al., 1988). Furthermore, recent reanalyses have brought the results from observational and randomized studies into line. Vandenbroucke and Psaty (2008) claim that observational studies may better reflect the true harm in real-life than selected populations enrolled in RCTs.

\subsection{Conclusion}

This chapter provided insight in the added value of user-driven design for the development of websites in the health care domain. Our research demonstrated that investigating the way end users work with a medium resulted in an improved communication means that enables them to retrieve relevant information more efficiently, effectively, and with higher satisfaction. This chapter provides the steps for dynamically communicating clinical practice guidelines and positively affecting elaboration of information, which is considered to be most effective for learning. Furthermore, the chapter shows how to undertake usability testing in order to elicit user input for usability improvement of existing communication means. 
In February 2008, the website was officially launched online. After its implementation, we summatively evaluated the website in several studies, which are described in the three following chapters. The first summative evaluation, which is elaborated on in this chapter, involved the website's usability compared to other websites about Methicillin Resistant Staphylococcus aureus (MRSA). Second, we investigated to what degree health care workers (HCWs) had incorporated the website into daily work practice and which factors affected adoption of the website (see Chapter 6). Third, we studied the determinants of adherence to the infection control guidelines communicated by the website (see Chapter 7).

The purpose of the study reported in this chapter was no longer to test the usability of the website itself as was the case in the previous chapter but rather to inquire into the functioning of the website in the "real world". In other words, this chapter reports the evaluation of the implemented website on the "open Internet", whereas the study reported in the previous chapter was conducted within a closed domain. Although HCWs from three different occupational groups were involved in the studies reported in the previous and subsequent chapters, the current study focuses on nurses. Nurses were chosen because preliminary results of the functioning of the website in daily practice (see Chapter 6) showed that the website was predominantly used by nurses.

Twenty nurses used the Internet to seek guidelines to solve MRSA-related scenarios, while thinking aloud. The scenarios had already been used in the studies presented in Chapter 4. Nurses' information-seeking skills appeared rather basic, implying a limited amount of search terms and websites consulted. Ineffective searches were mainly caused by a mismatch between the retrieved guidelines and nurses' tacit knowledge. The website developed for this thesis's research appeared to generate fewer mismatch problems compared to other websites. Perceived practical relevance and information completeness were the most applied quality criteria. The website under investigation better fulfilled these criteria compared to other websites. Accuracy and disclosures, whose importance is emphasized in existing e-health literature, were considered less important among nurses. As long as information matched their practical and experiential knowledge, they were satisfied, even when information was incorrect. This is dangerous because nurses' reliance on incorrect information might result in inadequate behavior and/ or medical complications. Accuracy and disclosures of websites of national authorities such as the Dutch Working Group on Infection Prevention were perceived as being better compared the website developed for this thesis's research, implying that the credibility of the national authorities' websites were valued better than the website under investigation.

Since this chapter has been submitted to an international scientific journal, the results are presented as general recommendations for website design. Within our research project however, the results of the study were used to revise the content, structure, and presentation of the website. 


\section{Chapter 5 \\ How nurses seek and evaluate clinical practice guidelines on the Internet}

Based on: Verhoeven F, Steehouder MF, Hendrix MGR, Gemert-Pijnen JEWC van. How nurses seek and evaluate clinical practice guidelines on the Internet. Accepted for publication in Journal of Advanced Nursing. 


\section{Chapter 2}

Assess and analyze needs

Chapters $3 \& 4$

Rank and select needs

Chapters 3 \& 4

Identify solutions,

Articulate goals

Chapters 3 \& 4

Design and development

\section{Chapter 5}

Implement solution

\section{Chapter 6}

Users adopt solution

\section{Chapter 7}

Users realize goals 


\section{How nurses seek and evaluate clinical practice guidelines on the Internet}

\subsection{Introduction}

The interactive nature of the Internet combined with its potential to store large volumes of information offers a unique opportunity to provide nurses with highquality evidence-based guidelines, irrespective of time and place (Roy et al., 2006). Previous research has demonstrated that a website enables nurses to seek clinical practice guidelines more efficiently and effectively than paper-based resources (see Chapter 4).

So far, it appeared that nurses mainly use the Internet for their own study purposes. Trying to find an answer to clinical questions encountered in daily practice is a less common reason for nurses to use the Internet (Gosling et al., 2003). Given that the evidence-based healthcare movement has largely rejected the traditional textbook as a source of up-to-date clinical knowledge, and given that nurses are increasingly taking on responsibilities traditionally regarded as the province of doctors, it becomes ever more important for nurses to be able to use the Internet to access up-to-date clinical practice guidelines (McCaughan et al., 2005; Wozar \& Worona, 2003).

\subsection{Background}

Limited Internet use to retrieve clinical practice guidelines among nurses can be attributed to individual factors (limited skills, technophobia), website characteristics (perceived slowness, poor design), and organizational factors (inability to access, lack of time) (McCaughan et al., 2005). Nurses are more likely to value interpersonal contact, and they prefer to use personal experience and communication with colleagues and patients rather than consult the Internet in order to take clinical decisions (Estabrooks et al., 2003).

For nurses to perceive a website as valuable, energy should be focused on constructing websites that dovetail with nurses' tacit knowledge and needs. Numerous criteria exist that guarantee the quality of health-related websites, including accuracy, completeness, readability, disclosures, and references (Eysenbach et al., 2002, Griffiths \& Christensen, 2005). However, these criteria mainly reflect web experts' concerns, and little is known about nurses' perspectives regarding these items, though we do know that users and experts generate different criteria for assessing the quality of 
information materials (Coulter, 1998). Therefore, nurses' tacit knowledge, which is context-dependent and made up of the practical and experiential wisdom of individual nurses, should be considered when developing websites for the communication of clinical practice guidelines (Williams \& Dickinson, 2008). The websites should also be designed for nurses who may not have sophisticated information-seeking skills (Estabrooks et al., 2003).

In the current study, we studied nurses' behavior when searching for and evaluating clinical practice guidelines on the Internet. Our research questions each concern a phase of the information-seeking process (Marchionini, 1995) (see Table 5.1.):

1) How do nurses search for clinical practice guidelines on the Internet?

2) What problems do nurses encounter when they search for clinical practice guidelines on the Internet?

3) How do nurses evaluate clinical practice guidelines they retrieved on the Internet and the website providing the guidelines?

4) To what degree are nurses able to successfully retrieve clinical practice guidelines on the Internet?

Based on the results, we provide specific design recommendations that address nurses' needs for web-based communication of clinical practice guidelines. 
Table 5.1. Conceptual framework to determine nurses' information-seeking strategies, problems encountered, evaluation criteria, and search outcomes when seeking clinical practice guidelines on the Internet

Formulate and execute query, examine results

\begin{tabular}{|c|c|c|c|c|c|}
\hline $\begin{array}{l}\text { 1. Information- } \\
\text { seeking strategy }\end{array}$ & $\begin{array}{l}\text { 2. Problems } \\
\text { encountered } \\
\text { during search }\end{array}$ & $\begin{array}{l}\text { 3. Evaluation } \\
\text { criteria }\end{array}$ & $\begin{array}{l}\text { 4. Search out- } \\
\text { comes }\end{array}$ & Definition & $\begin{array}{l}\text { Type of } \\
\text { data* }\end{array}$ \\
\hline Orienteering & & & & $\begin{array}{l}\text { Reaching the target website in a series of } \\
\text { steps, without specifying the entirety of the } \\
\text { information needed up front }\end{array}$ & 0 \\
\hline \multirow[t]{17}{*}{ Teleporting } & & & & Jumping directly to the target website & 0 \\
\hline & $\begin{array}{l}\text { Problematic } \\
\text { query formula- } \\
\text { tion }\end{array}$ & & & $\begin{array}{l}\text { Difficulties that are encountered with the } \\
\text { translation of the scenario into a meaningful } \\
\text { query }\end{array}$ & V \\
\hline & $\begin{array}{l}\text { Semantic mis- } \\
\text { match }\end{array}$ & & & $\begin{array}{l}\text { Mismatch between the vocabulary used by } \\
\text { the search engine's database and nurses' ter- } \\
\text { minology or discrepancy between expected } \\
\text { and retrieved clinical practice guidelines }\end{array}$ & V \\
\hline & $\begin{array}{l}\text { Lack of (tacit) } \\
\text { knowledge }\end{array}$ & & & $\begin{array}{l}\text { Lack of (tacit) knowledge that negatively af- } \\
\text { fects the way search results are evaluated }\end{array}$ & V \\
\hline & $\begin{array}{l}\text { Incorrect (tacit) } \\
\text { knowledge }\end{array}$ & & & $\begin{array}{l}\text { Tacit knowledge that appears to be incor- } \\
\text { rect and negatively affects the way query } \\
\text { is formulated and/ or the way results are } \\
\text { evaluated }\end{array}$ & 0 \\
\hline & & Accuracy & & $\begin{array}{l}\text { Information provided by the website is in } \\
\text { concordance with the best available evidence }\end{array}$ & V \\
\hline & & Completeness & & $\begin{array}{l}\text { Necessary parts, elements, or steps to make } \\
\text { a decision for daily practice, provided by } \\
\text { the website }\end{array}$ & V \\
\hline & & Readability & & $\begin{array}{l}\text { Complexity and lengthiness of sentences } \\
\text { of the provided information on the website } \\
\text { (excluding design aspects such as font size) }\end{array}$ & V \\
\hline & & $\begin{array}{l}\text { References } \\
\text { provided }\end{array}$ & & $\begin{array}{l}\text { References to other information sources } \\
\text { provided on the website }\end{array}$ & V \\
\hline & & Disclosures & & $\begin{array}{l}\text { Information about terms of use, copyright, } \\
\text { links to other sites, and liability, provided by } \\
\text { the website }\end{array}$ & V \\
\hline & & Relevance & & The practical applicability of the information & V \\
\hline & & Design & & $\begin{array}{l}\text { Visual aspects of the website layout (includ- } \\
\text { ing design aspects such as font size) }\end{array}$ & V \\
\hline & & Usability & & $\begin{array}{l}\text { The effectiveness, efficiency, and satisfac- } \\
\text { tion with which the website can be used to } \\
\text { retrieve clinical practice guidelines }\end{array}$ & V \\
\hline & & Findability & & $\begin{array}{l}\text { The ease with which a particular website can } \\
\text { be discovered or located on the Internet }\end{array}$ & V \\
\hline & & & Time & $\begin{array}{l}\text { Efficiency: Number of seconds needed to } \\
\text { complete a scenario }\end{array}$ & 0 \\
\hline & & & Effort & $\begin{array}{l}\text { Efficiency: Number of actions needed to } \\
\text { complete a scenario: mouse-clicks, entering } \\
\text { a search term, etc. }\end{array}$ & $\mathrm{O}$ \\
\hline & & & Completion & $\begin{array}{l}\text { Effectiveness: Scenario completed success- } \\
\text { fully or not }\end{array}$ & $\mathrm{O}$ \\
\hline
\end{tabular}

\footnotetext{
${ }^{*} \mathrm{O}=$ observation; $\mathrm{V}=$ verbalization
} 


\subsection{The study}

\subsubsection{Aim}

The aim of the study was first to assess nurses' information-seeking strategies and problems encountered when seeking clinical practice guidelines on the Internet, and next, to investigate the criteria they apply when evaluating the guidelines and the websites communicating the guidelines, in order to supply design recommendations for websites with clinical practice guidelines.

\subsubsection{Sample}

Due to increased international trade in health services, Methicillin Resistant Staphylococcus aureus (MRSA) is one of the organisms that most commonly contribute to the rapid spread of health care-associated infections (Friedrich et al., 2008). The Dutch-German research project EUREGIO MRSA-net Twente/Münsterland addresses this problem at a cross-national level, and the study reported here was conducted as part of this project. Respondents were therefore asked to look for MRSA-related guidelines.

The convenience sample involved 20 nurses, all of whom were employed in a hospital in the West Netherlands. Respondents were recruited via intranet postings and posters displayed in the hospital. Eligible respondents were Dutch speakers and had experience with Internet use. The majority of the respondents were female $(n=15$; $75 \%)$, and their mean age was 34.9 years $(S D=9.6)$. On average, respondents had been in their present job for 6.6 years $(S D=7.5)$, and in their line of work for 8.7 years $(S D=7.4)$. Most respondents worked in a surgery unit $(n=7 ; 35 \%)$, in a paediatric ward $(n=3 ; 15 \%)$, in traumatology $(n=2 ; 10 \%)$, in a diabetic clinic $(n=2 ; 10 \%)$ or on another ward $(n=6 ; 30 \%)$. Thirteen respondents $(65 \%)$ completed an intermediate vocational education, six $(30 \%)$ had a higher vocational education, and one respondent $(5 \%)$ held a master's degree in Advanced Nursing Practice. Data collection took place between July and August of 2008.

Since the target population was homogenous, and we were interested in identifying the behavior of only one occupational group, a sample size of $\mathrm{N}=20$ was satisfactory (U.S. Department of Health and Human Services, 2009).

\subsubsection{Data collection}

Twenty nurses were asked to complete simulated MRSA-related scenarios using the Internet. Scenario-based tests involve users participating in simulated scenarios based on clearly defined tasks. This technique has previously been established and used successfully to test clinical decision-making (Avery et al., 1980; Van GemertPijnen et al., 2005; Kolbe et al., 1996; Nielsen 1994). Scenarios can vary in their level of detail: task-based (which only state what the user should do without providing any information on how the user should complete the scenario), elaborated (more detailed user stories), or full-scale (which include the steps to accomplish the scenario) (U.S. 
Department of Health and Human Services, 2009). Since our purpose was to let nurses show us how they would use the Internet to accomplish a scenario, we used taskbased scenarios. Respondents were asked to complete scenarios using the Internet or the hospital intranet. We formulated 30 scenarios, such as: "You are attending to an MRSA patient in an isolation room. Which preventive measures must you take in order not to transmit MRSA to other patients or personnel?"

The respondents were asked to constantly verbalize their thoughts during testing. We used the procedure for the thinking-aloud technique proposed by Boren and Ramey (2000). Each time respondents ended a statement with "a stretched, interrogative intonation contour" thereby creating a "slot" for an acknowledgment token, the test administrator used the reminder "mm-hmm" with a rising intonation. This token was also produced when the respondent fell silent. In addition, when a respondent was unclear, the test administrator asked for clarification. Also, when a respondent was stuck, the test administration encouraged the respondent to continue and offered indirect suggestions of how to do so. This so-called thinking-aloud method has high face validity since the data obtained reflect the actual user behavior (Van den Haak, 2008).

No time limit was imposed on the respondent to work on a scenario, and for each scenario we recorded whether or not it was completed successfully (effectiveness), the number of seconds spent navigating to the answer (efficiency), and the number of actions it took a respondent to complete a scenario (efficiency) (Frøkjær, 2000).

The study was conducted in a silent, separate room in the hospital on a laptop with an Internet connection and the Internet Explorer web browser with a blank start page. Each test lasted about one hour. The respondents' activities on the laptop and verbalizations during scenario performance were recorded with screen capture software (Camtasia ${ }^{\mathrm{TM}}$ ). All data were transcribed verbatim afterwards.

\subsubsection{Ethical considerations}

The study was approved by the management of the hospital in which all respondents were employed. No formal approval of a Research Ethics Committee was required, since our study did neither directly affect respondent's wellbeing, nor could any disclosure of the subjects' response outside the study reasonably place the respondent at risk of civil liability, financial standing, employability, or reputation. Software monitoring of the Internet activity operated continuously and invisibly to the respondents and therefore did not alert or remind the respondents that their activity was being monitored. Respondents gave oral consent to participate. We emphasized that the respondents' contribution would be strictly confidential.

\subsubsection{Data analysis}

When the twenty tests were completed, we produced transcripts of all verbalizations and noted the respondent's navigation path. Furthermore, we recorded the observational data for each scenario (see Table 5.1.) during data transcription: 
whether or not it was completed successfully (effectiveness), the number of seconds spent navigating to the answer (efficiency), the number of actions it took a respondent to complete a scenario (efficiency), and the information-seeking strategy (Frøkjær et al., 2000; Van den Haak 2008).

Verbal data from one randomly selected transcribed scenario-based test were analyzed independently by two researchers using deductive analysis, implying that the coding categories were derived from the conceptual framework (see Table 5.1) (Patton, 1990). Disagreement was solved by discussion after jointly reviewing the statement. Subsequently, all transcribed data were coded by the principal investigator. Then the total number of statements was collected for each coding category, which resulted in a total of 1063 coded statements. 333 codes involved problems encountered and 730 concerned evaluation criteria (see Table 5.1.). Repeated or reworded descriptions of the same category were only counted once per scenario. After coding, (nearly) synonymous statements concerning the evaluation criteria were combined into more general subthemes in order to create a better-organized overview of the results. The subthemes that emerged are presented in Tables 3 and 4 in the Results section.

Independent Samples T Tests using SPSS 16.0 were conducted to verify whether the differences in time and effort for both successfully completed and not successfully completed scenarios were significant.

\subsubsection{Validity and reliability}

The scenarios (see Table 5.2.) were used in earlier research (Van Gemert-Pijnen, 2005; see Chapter 4) and were validated by a Dutch medical microbiologist, who also defined the correct scenario outcomes and determined whether respondents' tacit knowledge was either correct or incorrect. A scenario was successfully completed if a nurse found the right solution without help from the test administrator.

Since the data set was too large to have it reviewed independently by two researchers, a random selection of $5 \%$ of all coded statements was coded by a second independent researcher. The relative strength of agreement (Cohen's Kappa $=0.84$ ) can be described as "almost perfect" in terms of Landis and Koch (1977).

\subsection{Results}

\subsubsection{How do nurses search for clinical practice guidelines on the Internet?}

Nurses either started the information-seeking process by teleporting (directly jumping to a website) or by orienteering (reviewing contextual information to narrow in on the information sought, often in a series of steps). In 71 out of 175 scenarios (40.6\%), respondents began by teleporting to either the hospital intranet or the website www. mrsa-net.nl. Teleporting mainly occurred when the respondent remembered having 
read about the topic earlier while solving a previous scenario (28 statements), used tacit knowledge to formulate a specific query ( 17 statements), or visited a particular website with which he or she was already acquainted (nine statements). The latter mostly happened when nurses sought information about risk factors to acquire MRSA, in scenarios concerning protective measures, and in scenarios concerning MRSA and health care workers' own health (see Table 5.2.):

"I'll go to ConsuMed. A website that I use more frequently. Because it enables you to search for diseases or medicines or homeopathic."

\section{(Nurse radiology department)}

In 104 of 175 scenarios (59.4\%), respondents had no specific idea about where to find the required information, or they did have an idea, but failed to recall the relevant website. Respondents then started orienteering by entering a query in Google. In 73 scenarios $(70.2 \%)$, one query was entered, and in the remaining scenarios, two or more queries were entered (with a maximum of seven words).

Twelve respondents ( 31 statements) used more sophisticated search options, like Google's "Advanced search" possibility. However, in the majority of scenarios such search strategies were not employed, and 11 respondents explicitly mentioned having insufficient information-seeking skills:

"Maybe I should enter two words in the search engine. Maybe that's more practical. I'm not good at these things, looking up... uhm, What can I try?"

\section{(Nurse diabetic outpatient clinic)}

After entering a query in Google, most respondents (in 71 of 104 scenarios, 68.3\%) selected only one hit generated by the search engine. This was most often the website ranked first among the Google results ( 45 scenarios, $63.4 \%$ ) or the second ( 11 scenarios, $15.5 \%)$. In 21 out of 104 scenarios $(20.2 \%)$, two hits were selected from the result list, and in the remaining 12 scenarios, three or more (11.5\%). Here, it appeared that respondents did not have a particular reason to select a hit from Google. Five times respondents indicated that they selected a website after having read the summary texts provided by Google.

\subsubsection{Which problems do nurses encounter when they search for clinical practice guidelines on the Internet?}

Five types of problems emerged during the information-seeking process, which can be found in the right column of Table 5.2. Each of the problem types will be discussed below. 


\section{Problems with formulating query (FOR)}

Twelve respondents encountered problems when translating the scenario into a meaningful query because they could not think of an appropriate search term. Scenarios that generated several problems concerned decolonization therapy (four statements), admission of a foreign patient (four statements) and what to do when the intensive care unit (ICU) is closed due to an MRSA-outbreak (five statements).

\section{Mismatch during examine results-phase (MER)}

Sixty times, respondents $(n=19)$ encountered matching problems when examining the hits resulting from a search engine, which implies a discrepancy between the respondent's expectation of the search results after entering a query and the results actually generated by the search engine. This occurred 27 times with Google, 19 times with the hospital intranet search engine, seven times with the search engine of www.mrsa-net.nl and seven times on other websites. Google particularly produced irrelevant results concerning admission of a foreign patient (eight statements).

\section{Mismatch during reflect-iterate-stop-phase (MRIT)}

In 94 of the 111 successfully completed scenarios (84.7\%), the answer retrieved by the respondents matched their expectations. However, mismatches occurred in 62 scenarios during the reflect-iterate-stop-phase. Of these, 16 involved the hospital intranet, seven involved www.mrsa-net.nl, and the remainder referred to other websites. The mismatch incidents mainly involved the scenario about risk factors for acquiring MRSA (ten statements), communication procedures in case of MRSA ( 11 statements), and the risk for pregnant nurses of attending an MRSA patient ( 11 statements). Again, it were the more specific precautions that generated problems. Encountering mismatch problems sometimes produced feelings of annoyance since it extended the search duration ( 14 scenarios, of which five were encountered while using the hospital intranet, and six times it concerned downloading a Portable Document Format [PDF]).

\section{Lack of knowledge (LOK)}

In 17 scenarios (ten respondents), a lack of (tacit) knowledge or experience impeded the respondent in assessing whether the retrieved information matched the scenario. This mainly concerned the scenarios about what to do when one's partner (private) is MRSA-positive (four statements), MRSA and pigs (four statements), and the admission of a foreign patient (four statements): 
"I didn't know much about it, actually. That with pig farmers, that they have an increased risk of acquiring MRSA... that doesn't really occur in this region. (...) I was not aware of it, so I cannot assess whether this information is complete or not."

(Nurse surgery ward)

\section{Incorrect knowledge (IK)}

In 17 scenarios (nine respondents), tacit knowledge that appeared incorrect resulted in retrieval of the wrong information, which was particularly the case for the scenarios about admission of a pig farmer (three statements), about an MRSA-positive partner (three statements), and about what to do in case of death of an MRSA patient (three statements):

"In theory, when a patient with MRSA is allowed to enter the hospital, then a nurse with MRSA is also permitted to, I assume."

(Nurse paediatric ward)

\subsubsection{How do nurses evaluate clinical practice guidelines they retrieved on the Internet and the website providing the guidelines?}

Tables 5.3. and 5.4. reflect the criteria nurses applied when evaluating web-based clinical practice guidelines and the websites providing the guidelines. Only criteria that were applied by at least two different respondents are included.

Overall, 730 statements about information and website quality were identified. Table 5.4. summarizes the number of statements separated for each quality criterion. Assuming that the criteria were mentioned on the respondents' own accord, completeness and relevance were the most salient criteria. Information completeness of the retrieved guidelines was particularly valued negatively, in contrast to information relevance. The frequency with which the other criteria were put forward was comparable, except for references provided and findability, which apparently were considered less important by the respondents.

\subsubsection{To what degree are nurses able to successfully retrieve clinical practice guidelines on the Internet?}

\section{Effectiveness}

A total of 111 out of $175(63.4 \%)$ scenarios were completed successfully. The successfully completed scenarios particularly involved the guidelines for MRSApositive staff, MRSA among pigs, patient admission, transfer, and discharge (see Table 5.2.). Unsuccessfully completed scenarios concerned the consequences of an MRSA-positive (private) partner, guidelines for pregnant nurses in relation to nursing 
an MRSA patient, and where to acquire more information about MRSA. In other words, universal precautions and background information concerning MRSA were identified successfully on the Internet in contrast to more specific precautions. In 70 scenarios (63.1\%), the answer was found on www.mrsa-net.nl. In 18 scenarios (16.2\%), the hospital intranet contained the right answer. Other websites where relevant information was retrieved varied considerably.

\section{Efficiency}

It took the respondents an average of 153.2 seconds $(S D=135.5$, range $6-856)$ to complete a scenario successfully. On scenarios that were not successfully completed, respondents spent a mean time of 236.0 seconds $(S D=127.0$, range 24-555). This difference was significant ( $p<.001$ ). The respondents required on average 7.8 actions to finish unsuccessfully completed scenarios, and 6.3 actions to finish successfully completed scenarios ( $p<.05)$.

Table 5.2. shows that particularly the scenarios involving background information and general precautions were solved rather quickly compared to the more specific scenarios about treatment, communication procedures, and nurses' own health. It took the respondents over 200 seconds on average to retrieve the more specific precautions. 
Table 5.2. Nurses' search strategies and problems that emerged when using the Internet to seek web-based clinical practice guidelines $(\mathrm{N}=20)$

\begin{tabular}{|c|c|c|c|c|c|c|}
\hline Category & Scenario & 总 & 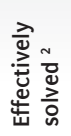 & 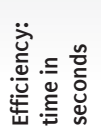 & 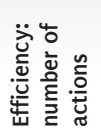 & $\frac{\sum^{n}}{\frac{n}{0}}$ \\
\hline \multirow{3}{*}{$\begin{array}{l}\text { MRSA in } \\
\text { general }\end{array}$} & 1. Risk factors for acquiring MRSA & $\mathrm{T}$ & $5 / 10$ & 171 & 9 & MER/MRIT \\
\hline & 2. Spread of MRSA & $\mathrm{O}$ & $1 / 1$ & 41 & 3 & \\
\hline & 3. MRSA among pigs & 0 & $8 / 10$ & 125 & 5 & LOK \\
\hline \multirow[t]{3}{*}{ Screening } & 4. Surveillance cultures in general & $\mathrm{O}$ & $2 / 5$ & 159 & 8 & MER \\
\hline & 5. Take swabs from patient & $\mathrm{T}$ & $5 / 10$ & 209 & 7 & MER/MRIT/LOK \\
\hline & 6. Take swabs from staff & $\mathrm{O}$ & $3 / 3$ & 149 & 9 & \\
\hline \multirow{4}{*}{$\begin{array}{l}\text { Protective } \\
\text { measures }\end{array}$} & 7. Tasks and responsibilities in case of an MRSA outbreak & $\mathrm{T}$ & $6 / 9$ & 284 & 7 & MER/ MRIT \\
\hline & 8. Measures to prevent transmission of MRSA & $\mathrm{T}$ & $8 / 10$ & 193 & 7 & MRIT \\
\hline & 9. Protective clothing & O & $1 / 1$ & 81 & 2 & \\
\hline & 10. An MRSA-positive patient at haemodialysis & 0 & $1 / 1$ & 160 & 12 & \\
\hline \multirow{3}{*}{$\begin{array}{l}\text { Disinfec- } \\
\text { tion } \\
\text { measures }\end{array}$} & 11. Contaminated items with MRSA patients' blood & 0 & $1 / 2$ & 76 & 2 & \\
\hline & 12. Unprotected contact with an MRSA patient & $\mathrm{O}$ & $3 / 3$ & 257 & 12 & \\
\hline & 13. Contact with an MRSA patient, no protective clothing & 0 & $1 / 1$ & 132 & 4 & \\
\hline \multirow[t]{5}{*}{ Admission } & 14. Patient from an institution with recent MRSA outbreak & 0 & $2 / 2$ & 127 & 2 & \\
\hline & 15. Patient, MRSA-positive four months ago & O & $2 / 2$ & 176 & 9 & \\
\hline & 16. Foreign patient (from Germany) & $\mathrm{T}$ & $6 / 10$ & 164 & 5 & FOR/ MER/ LOK \\
\hline & 17. Pig farmer & 0 & $1 / 2$ & 141 & 10 & MRIT/IK \\
\hline & 18. Patient, previously hospitalised in a foreign country & $\mathrm{T}$ & $5 / 7$ & 163 & 9 & MER \\
\hline \multirow[t]{2}{*}{ Transport } & 19. Patient's protective clothing & 0 & $7 / 9$ & 143 & 7 & \\
\hline & 20. Patient transport from OR to ICU & 0 & $5 / 5$ & 137 & 6 & FOR \\
\hline \multirow[t]{2}{*}{ Discharge } & 21. MRSA-positive patient needs homecare & $\mathrm{T}$ & $7 / 8$ & 108 & 4 & \\
\hline & 22. Death of an MRSA-positive patient & 0 & $4 / 4$ & 156 & 9 & MER/IK \\
\hline \multirow[t]{3}{*}{ Treatment } & 23. Difference between nurses and physicians & 0 & $4 / 6$ & 110 & 7 & FOR \\
\hline & 24. Difference between staff and patients & $\mathrm{T}$ & $2 / 5$ & 226 & 9 & MER \\
\hline & 25. Medication for MRSA & O & $3 / 6$ & 214 & 8 & \\
\hline \multirow{3}{*}{$\begin{array}{l}\text { MRSA and } \\
\text { your own } \\
\text { health }\end{array}$} & 26. MRSA-positive (private) partner & $\mathrm{T}$ & $3 / 10$ & 210 & 5 & MRIT/ LOK/ IK \\
\hline & 27. MRSA-positive yourself (as a nurse) & $\mathrm{T}$ & $7 / 8$ & 199 & 6 & MRIT \\
\hline & 28. Pregnant nurses and MRSA-positive nurses & 0 & $3 / 9$ & 225 & 7 & MER/ MRIT \\
\hline \multirow{2}{*}{$\begin{array}{l}\text { Communi- } \\
\text { cation }\end{array}$} & 29. Communication process in case of an MRSA outbreak & O & $3 / 8$ & 324 & 8 & FOR/ MER/ MRIT \\
\hline & 30. Whom to contact in case of MRSA? & $\mathrm{T}$ & $2 / 8$ & 177 & 7 & MER/ MRIT \\
\hline
\end{tabular}

${ }^{1} \mathrm{~T}$ =teleporting, $\mathrm{O}=$ orienteering. Search strategy used in majority of performed scenarios.

${ }^{2}$ E.g., Scenario 1 was performed by ten respondents. Five of them completed the scenario successfully, five of them did not.

${ }^{3} \mathrm{FOR}=$ problems with formulating query; $\mathrm{MER}=$ mismatch during examine results-phase; $\mathrm{MRIT}=$ mismatch during reflect-iterate-stop; $\mathrm{LOK}=$ lack of (tacit) knowledge; IK= incorrect (tacit) knowledge. Problems are reported here when they were encountered by at least four respondent performing the scenario. MER, MRIT, and LOK were verbalized by respondents, IK was observed by the researcher. 
Table 5.3. Nurses' evaluation criteria applied when using the Internet to seek clinical practice guidelines, resulting from thinking-aloud data $(\mathrm{N}=20)$

\begin{tabular}{|c|c|c|c|c|c|}
\hline & Category & & Criteria resulting from thinking-aloud data & \#st. ${ }^{1}$ & ${ }^{*}$ res. $^{2}$ \\
\hline \multirow{31}{*}{ 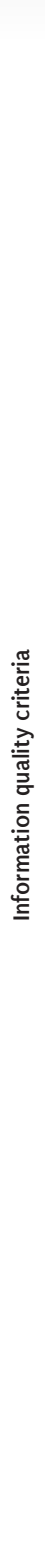 } & \multirow[t]{4}{*}{$\begin{array}{l}\text { Accuracy }(+) \\
(52 \text { statements, } n=17)\end{array}$} & 1 & $\begin{array}{l}\text { Information communicated on the website corresponds with nurses' work } \\
\text { practice. }\end{array}$ & 22 & 12 \\
\hline & & 2 & Information communicated on the website matches nurses' tacit knowledge. & 24 & 12 \\
\hline & & 3 & Website mentions recent data that caused the information to be updated. & 3 & 3 \\
\hline & & 4 & $\begin{array}{l}\text { Website mentions that presented guidelines might differ from guidelines } \\
\text { locally enforced. }\end{array}$ & 3 & 2 \\
\hline & \multirow[t]{3}{*}{$\begin{array}{l}\text { Accuracy }(-) \\
(37 \text { statements, } n=14)\end{array}$} & 5 & $\begin{array}{l}\text { National guidelines communicated on the website do not correspond with } \\
\text { guidelines locally enforced. }\end{array}$ & 7 & 7 \\
\hline & & 6 & $\begin{array}{l}\text { Guidelines communicated on the website do not correspond with nurses' } \\
\text { work practice. }\end{array}$ & 20 & 13 \\
\hline & & 7 & Date of origin is outdated or even lacking. & 10 & 6 \\
\hline & \multirow{2}{*}{$\begin{array}{l}\text { Completeness }(+) \\
(50 \text { statements, } n=18)\end{array}$} & 8 & Information is comprehensive. & 36 & 14 \\
\hline & & 9 & Information is extensive, additional to basic information. & 14 & 10 \\
\hline & \multirow{2}{*}{$\begin{array}{l}\text { Completeness }(-) \\
(83 \text { statements, } n=19)\end{array}$} & 10 & More aspects are known from work practice but are not provided & 37 & 16 \\
\hline & & 11 & Practical information is lacking. & 46 & 19 \\
\hline & \multirow{4}{*}{$\begin{array}{l}\text { Readability }(+) \\
(76 \text { statements, } n=20)\end{array}$} & 12 & Information is clear. & 58 & 19 \\
\hline & & 13 & Information is comprehensible (appropriate word use). & 9 & 6 \\
\hline & & 14 & Information provides indubitable precautions. & 6 & 6 \\
\hline & & 15 & Information is formulated clearly and concisely. & 3 & 3 \\
\hline & \multirow[t]{5}{*}{$\begin{array}{l}\text { Readability }(-) \\
(24 \text { statements, } n=12)\end{array}$} & 16 & Information is communicated in medical jargon. & 5 & 5 \\
\hline & & 17 & Information generates doubts. & 7 & 7 \\
\hline & & 18 & A step-by-step plan for work practice is lacking. & 3 & 3 \\
\hline & & 19 & Similar information is fragmentarily presented. & 2 & 2 \\
\hline & & 20 & Website contains too much text. & 7 & 6 \\
\hline & \multirow{3}{*}{$\begin{array}{l}\text { References }(+) \\
(17 \text { statements, } n=11)\end{array}$} & 21 & Inclusion of references is good. & 6 & 5 \\
\hline & & 22 & Inclusion of references enables nurse to look for other sources him/herself. & 3 & 2 \\
\hline & & 23 & Inclusion of references increases information credibility. & 8 & 6 \\
\hline & $\begin{array}{l}\text { References }(-) \\
(2 \text { statements, } n=2)\end{array}$ & 24 & The absence of references detracts the information credibility. & 2 & 2 \\
\hline & \multirow{3}{*}{$\begin{array}{l}\text { Disclosures }(+) \\
(49 \text { statements, } n=19)\end{array}$} & 25 & Source is or looks familiar and therefore information is perceived as credible. & 27 & 10 \\
\hline & & 26 & Presence of source disclosure increases information credibility. & 17 & 13 \\
\hline & & 27 & Information is targeted at nursing practice and therefore credible. & 5 & 4 \\
\hline & \multirow{4}{*}{$\begin{array}{l}\text { Disclosures (-) } \\
\text { (30 statements, } n=14)\end{array}$} & 28 & The website does not disclose which organization(s) developed the website. & 8 & 7 \\
\hline & & 29 & Source is not familiar to nurses and therefore information is not credible. & 5 & 4 \\
\hline & & 30 & Source is not credible for this type of information. & 5 & 5 \\
\hline & & 31 & $\begin{array}{l}\text { Website contains too many advertisements, at the expense of information } \\
\text { credibility. }\end{array}$ & 3 & 3 \\
\hline
\end{tabular}


Table 5.3. (continued) Nurses' evaluation criteria applied when using the Internet to seek clinical practice guidelines, resulting from thinking-aloud data $(\mathrm{N}=20)$

\begin{tabular}{|c|c|c|c|c|c|}
\hline & Category & & Criteria resulting from thinking-aloud data ${ }^{*}$ & \#st. ${ }^{1}$ & ${ }^{*}$ res. $^{2}$ \\
\hline \multirow{19}{*}{ 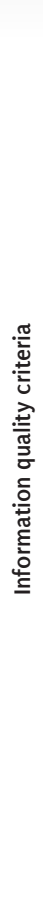 } & \multirow[t]{2}{*}{$\begin{array}{l}\text { Disclosures }(-) \\
\text { (30 statements, } n=14)\end{array}$} & 32 & $\begin{array}{l}\text { Website does not include a separate part for health care workers, at the } \\
\text { expense of information credibility. }\end{array}$ & 4 & 4 \\
\hline & & 33 & $\begin{array}{l}\text { There is no clear relationship between the source and official authorities in } \\
\text { the field. }\end{array}$ & 5 & 4 \\
\hline & \multirow{6}{*}{$\begin{array}{l}\text { Relevance }(+) \\
\text { (109 statements, } n=19)\end{array}$} & 34 & Information really concerns nursing practice. & 7 & 5 \\
\hline & & 35 & Information enables nurses to take a decision for practice. & 79 & 19 \\
\hline & & 36 & Information is practically-oriented. & 11 & 9 \\
\hline & & 37 & Information represents questions from daily practice. & 4 & 4 \\
\hline & & 38 & Website contains information that the nurse had not thought about before. & 4 & 4 \\
\hline & & 39 & Information includes personal relevance/risks for nurses. & 4 & 3 \\
\hline & \multirow{3}{*}{$\begin{array}{l}\text { Relevance }(-) \\
\text { (34 statements, } n=17 \text { ) }\end{array}$} & 40 & Information does not concern nursing practice. & 9 & 7 \\
\hline & & 41 & Information does not enable nurses to take a decision for practice. & 17 & 13 \\
\hline & & 42 & Information is not practically-oriented, only providing theory. & 8 & 8 \\
\hline & \multirow{4}{*}{$\begin{array}{l}\text { Design }(+) \\
\text { Website quality criteria } \\
\text { (40 statements, } n=19 \text { ) }\end{array}$} & 43 & Use of soft colors. & 7 & 5 \\
\hline & & 44 & Simple design without unnecessary frills. & 6 & 5 \\
\hline & & 45 & Professional design. & 12 & 10 \\
\hline & & 46 & Clear design. & 15 & 11 \\
\hline & \multirow{4}{*}{$\begin{array}{l}\text { Design }(-) \\
\text { (28 statements, } n=14)\end{array}$} & 47 & Dull design. & 12 & 7 \\
\hline & & 48 & Too few headings. & 7 & 5 \\
\hline & & 49 & Inappropriate color use. & 5 & 3 \\
\hline & & 50 & Small font. & 4 & 4 \\
\hline \multirow{4}{*}{ …... } & \multirow{5}{*}{$\begin{array}{l}\text { Usability }(+) \\
\text { (5 } 1 \text { statements, } n=20 \text { ) }\end{array}$} & 51 & Website (search engine) enables nurses to search for information easily. & 17 & 10 \\
\hline & & 52 & Headings facilitate categorical search. & 8 & 6 \\
\hline & & 53 & Clear navigation. & 21 & 16 \\
\hline & & 54 & Two separate website entrances (for public and personnel) enhance usability. & 3 & 3 \\
\hline \multirow{8}{*}{ 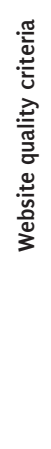 } & & 55 & (Nearly) Everything is clickable. & 2 & 2 \\
\hline & \multirow[t]{4}{*}{$\begin{array}{l}\text { Usability }(-) \\
\text { (44 statements, } n=17 \text { ) }\end{array}$} & 56 & $\begin{array}{l}\text { Portable Document Format does not allow nurses to search easily within the } \\
\text { document. }\end{array}$ & 9 & 7 \\
\hline & & 57 & Website structure does not match nurse's expectations. & 19 & 12 \\
\hline & & 58 & Search engine is lacking. & 6 & 5 \\
\hline & & 59 & $\begin{array}{l}\text { Several malfunctioning features, e.g., appearance of pop-ups, search engine } \\
\text { does not function properly, loading pages takes too long, etc. }\end{array}$ & 10 & 9 \\
\hline & $\begin{array}{l}\text { Findability }(+) \\
(2 \text { statements, } n=2)\end{array}$ & 60 & Website is easy to locate on the Internet since it is ranked highly in Google. & 2 & 2 \\
\hline & $\begin{array}{l}\text { Findability (-) } \\
\text { (2 statements, } n=2 \text { ) }\end{array}$ & 61 & $\begin{array}{l}\text { Website is not easy to locate on the Internet since it is not ranked highly in } \\
\text { Google. }\end{array}$ & 2 & 2 \\
\hline & \multicolumn{2}{|l|}{ Total } & & 730 & \\
\hline
\end{tabular}

* stated by at least two different respondents

' number of statements

${ }^{2}$ number of respondents 
Table 5.4. Number of positive and negative statements related to nurses' evaluation criteria when using the Internet to seek clinical practice guidelines on the Internet, drawn from thinking-aloud data $(\mathrm{N}=2 \mathrm{O})$

\begin{tabular}{|c|c|c|c|c|c|c|c|}
\hline & & \multicolumn{2}{|c|}{ Number of positive statements } & \multicolumn{2}{|c|}{ Number of negative statements } & \multicolumn{2}{|c|}{ Total number of statements } \\
\hline \multicolumn{2}{|c|}{ Criterion } & \# statements & \# respondents & \# statements & \# respondents & \# scenarios & \# respondents \\
\hline \multirow{6}{*}{ 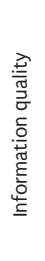 } & Accuracy & $52(11.7 \%)$ & 17 & $37(13.0 \%)$ & 14 & $89(12.2 \%)$ & 20 \\
\hline & Completeness & $50(11.2 \%)$ & 18 & $83(29.2 \%)$ & 19 & $133(18.2 \%)$ & 20 \\
\hline & Readability & $76(17.0 \%)$ & 20 & $24(8.5 \%)$ & 12 & $100(13.6 \%)$ & 20 \\
\hline & References & $17(3.8 \%)$ & 11 & $2(0.7 \%)$ & 2 & $19(2.6 \%)$ & 11 \\
\hline & Disclosures & $49(11.0 \%)$ & 19 & $30(10.6 \%)$ & 14 & $79(10.8 \%)$ & 20 \\
\hline & Relevance & $\log (24.4 \%)$ & 19 & $34(12.0 \%)$ & 17 & $143(19.6 \%)$ & 20 \\
\hline \multirow{4}{*}{$\begin{array}{l}\frac{\pi}{\bar{\sigma}} \\
\frac{0}{0} \\
0 \\
0 \\
. \frac{ \pm}{n} \\
\frac{0}{0} \\
3\end{array}$} & Design & $40(9.0 \%)$ & 19 & $28(9.9 \%)$ & 14 & $68(9 \cdot 3 \%)$ & 20 \\
\hline & Usability & $51(11.4 \%)$ & 20 & $44(15.5 \%)$ & 17 & $95(13.0 \%)$ & 20 \\
\hline & Findability & $2(0.4 \%)$ & 2 & $2(0.7 \%)$ & 2 & $4(0.5 \%)$ & 4 \\
\hline & Total & $446(100 \%)$ & & $284(100 \%)$ & & $730(100 \%)$ & \\
\hline
\end{tabular}




\subsection{Discussion}

This study substantially contributes to knowledge about nurses' information-seeking strategies and problems they encountered when seeking clinical practice guidelines on the Internet, next to criteria nurses apply when assessing the guidelines and the providing websites. Moreover, the study provides design recommendations for those responsible for communication of infection control guidelines.

\subsubsection{Limitations}

Because we used a sample comprised of nurses who were all employed in the same hospital, further research is needed to verify whether our findings are generalizable to other settings and occupational groups. Respondents voluntarily participated, possibly implying that they were motivated to successfully complete a scenario and their level of knowledge regarding clinical practice guidelines was possibly higher compared to the target population, which might have biased the results. However, considering the large number of problems our respondents encountered, the question arises regarding how many problems nurses less involved with the topic would experience.

Another limitation of our study was the laboratory setting, in that behaviors in the pursuit of actual clinical knowledge in a real clinical setting may be different from those shown in this controlled environment (Nielsen, 1993). However, the ability to use a defined set of scenarios provides a benefit that cannot be obtained in the real clinical setting.

Some doubts have been raised about validity and reliability of the thinking-aloud technique, but this critique focused on thinking aloud as a technique to study cognitive processes rather than to identify usability problems, which was the object of our study (Boren \& Ramey, 2000). We could have used other methods to assess nurses' information-seeking skills, like the E-health Literacy Scale (Norman \& Skinner, 2006). This is an eight-item measure of e-health literacy developed to measure consumers' combined knowledge, comfort, and perceived skills at finding, evaluating, and applying electronic health information to health problems. However, questionnaires fail to provide detailed information about exactly how the Internet is used, and only create a global impression.

\subsubsection{Answers to the research questions}

1) How do nurses search for clinical practice guidelines on the Internet?

Tacit knowledge determined which search terms nurses used and whether or not they had an explicit expectation of where to find the relevant information. The limited range and amount of simple search terms used, the low variety of websites visited, and the fact that sources were only selected based on convenience (ranked highly in Google), suggest that searches were unplanned, which is in line with previous findings (Morris-Docker et al., 2004). Websites should therefore simplify the search process 
and the accessibility of information.

2) Which problems do nurses encounter when they search for clinical practice guidelines on the Internet?

Five types of problems emerged during the information-seeking process. The majority of problems concerned a mismatch between the system's and the nurses' vocabulary. Furthermore, deficient tacit knowledge negatively affected the evaluation of information: relevant information was wrongfully evaluated as irrelevant and vice versa, which was also demonstrated by Eastin (2001).

3) How do nurses evaluate clinical practice guidelines they retrieved on the Internet and the website providing the guidelines?

Overall, our study emphasized that users and experts generate different criteria for assessing the quality of health-related websites.

Nurses in our sample particularly considered completeness and relevance as the most important information quality criteria. Table $5 \cdot 3$. Shows that information completeness is valued positively when it adds something to what is already known (criterion 9) and that information is assessed as incomplete when practical information related to nursing practice is lacking (criterion 11). The most salient reason to perceive information as relevant was when information enabled the nurse to take a decision for clinical practice (criterion 35), and the other way around for irrelevant information (criterion 41 ). Furthermore, information was found relevant if it contained new information (criterion 38) or if information was of personal relevance for patients or staff (criterion 39). Other criteria, such as accuracy and disclosures, whose importance is continuously emphasized by experts (Eysenbach et al., 2002; Kerr et al., 2006), were perceived as less important. This might imply danger for clinical practice, because when nurses rely on inaccurate information, they are putting themselves and patients at risk of potentially harmful interactions and adverse effects.

Tacit knowledge appeared to be a recurring theme in each criterion (see Table 5.3.). Respondents thought information was accurate when it matched their practical and experiential wisdom, in contrast to experts who define accuracy as "the degree of concordance of the information provided with the best available evidence" (Eysenbach et al., 2002). Nurses considered information complete when it added practical issues to what they already knew ("What is in it for me?"), whereas experts label completeness as "the degree to which all key elements are present" (Eysenbach et al., 2002). Experts take into account disclosure of authorship, purpose statement, and date of last update when assessing credibility; nurses were less critical regarding disclosures and valued a source as credible if they had prior experience with it and its information matched their tacit knowledge. This implies that those responsible for guideline communication should communicate correct guidelines in such a way that they match nurses' tacit knowledge. In this way, nurses will understand how to adequately perform clinical practice guidelines. The influence of tacit knowledge 
on the information-seeking process was previously demonstrated by Leonard and Sensiper (1998). They claimed that in problem solving (information-seeking), individuals overlay a problem with patterns derived from experience in order to quickly find a solution, and in problem finding (evaluation of the retrieved information), tacit knowledge is used to frame a problem. Incorporating tacit knowledge is vital to the development of information systems: the most advanced computer- or web-based information systems on their own cannot generate new knowledge. Only human beings who have accumulated practical and experiential wisdom have the capacity to generate new knowledge.

Readability, design, and accessibility of the website were of substantially less importance than relevance and completeness, according to nurses. As long as information was personally relevant and particularly aimed at nursing practice, nurses were satisfied. This practical-oriented information need was also found by Zhang (2005), and might result from the fact that nurses are most often the clinicians who inform and instruct patients about medical topics. It therefore seems logical that they would want to access practically-oriented medical information (Wozar \& Worona, 2003).

It appeared that the website that was rated most usable, nice, and practically relevant was perceived as less credible, and the websites with credible sources (such as the hospital intranet) were less usable and practically-oriented. A solution to improve credibility is by applying for certification. The Health on the Net Foundation (2009) and the Dutch ZegelGezond (2009), for instance, have developed an accreditation system to improve the quality of medical and health information online. The success of these systems has not yet been clearly determined, however, and they have been criticized for having little commitment among the general public (Rippen \& Risk, 2000).

The finding that nurses from our sample did not verify the accuracy and disclosures of the guidelines might be explained by the concept of "dynamism": when message presentation is found to be highly dynamic, perceptions of source credibility are elevated. It could be argued that a highly dynamic site could cause the respondents to perceive the content as more credible. In addition, the format or layout of the website could also raise perceived credibility (Hamilton, 1998).

4) To what degree are nurses able to successfully retrieve clinical practice guidelines on the Internet?

Overall, our respondents retrieved correct information in $63 \%$ of cases, which is comparable to findings from Hersh et al. (2002). It appeared that background information and universal precautions were identified more effectively and efficiently compared to more specific information regarding nurses' own health and communication procedures. This implies that creators of web-based guideline communication should simplify the search process to identify specific precautions, for instance by adding synonyms used by nurses to the content management system or by assigning informal tags to the guidelines, which help describe the guidelines in nurses' vocabulary and allows the guideline to be found again by browsing and 
searching (Smith, 2007).

It took nurses in our study an average of 153 seconds to successfully complete a scenario, which is relatively fast compared to Hersh's findings, who reported that it takes a health care provider more than 30 minutes to search for answers from the MEDLINE search engine. Compared to paper-based guidelines, searches were also relatively efficient (Verhoeven et al., 2009c). However, more specific guidelines, such as the risk of nursing an MRSA patient when pregnant took more time: over 200 seconds more, on average (3.3 minutes). Given that in reality, health care workers generally abandon a search after about two minutes, the efficiency with which specific clinical information was found on the Internet in our study can be rated as relatively low (Yu et al., 2007).

\subsubsection{Practical implications}

Based on our study, we provide the following design recommendations for a website with clinical practice guidelines:

\section{Simplify information-seeking process}

- Facilitate teleporting with navigation: Synonyms used by nurses should be added via a flexible content management system or by assigning tags, categorizing information according to nurses' mental models, and not providing information in a Portable Document Format.

- Enhance the website's findability in Google, and ensure that the website is among the top three hits.

- Include guidelines aimed at specific clinical situations next to universal precautions.

\section{Improve information quality}

- Information should fit nurses' tacit knowledge. (see Table 5.3., criteria 2, 10, 13, 15, 18)

- Information should fit nurses' current perception of the environment (provide practical issues). (see Table 5.3., criteria 1, 4, 25, 27, 34, 36, 37).

- Provide additional, practical information to nurses' tacit knowledge. (See Table 5.3., criteria 9, 14, 15, 35, 38, 39, 42)

- Guarantee information credibility by referring to authorities that nurses perceive to be credible. (See Table 5.3., criteria 3, 22, 23, 26, 31, 32 )

\section{Increase website quality}

- Consider standard usability criteria such as avoiding cluttered displays, using fluid layouts and moderate white space, etc. (Koyani et al., 2006), since nurses do not attach major importance to design and usability aspects.

- These design recommendations enhance the provision of targeted, evidence-based medical information to meet the specific needs of caregivers, supported by a sophis- 
ticated information technology infrastructure, which is called Information Therapy, advocated by the Institute of Medicine to improve the quality of care (Gwinn \& Seidman, 2007).

\subsection{Conclusion}

Nurses appeared to rely on tacit knowledge rather than to use advanced searching strategies when seeking clinical practice guidelines on the Internet. It is therefore essential to dovetail with nurses' specific needs by targeting guideline communication to nurses' tacit knowledge, providing additional information to what is already known, and guaranteeing credibility by referencing well-known authorities.

However, merely applying the aforementioned design recommendations is not sufficient to stimulate nurses to search for clinical practice guidelines on the Internet. Adoption of a website is not only determined by information and website quality, but there are also individual factors (e.g., attitude, knowledge, and job satisfaction) and contextual factors (e.g., communication, training, and management values) that have to be considered (Kukafka et al., 2003). Developing targeted adoption-enhancing interventions and their empirical evaluation is of vital importance.

Considering nurses' limited information-seeking skills, particularly with regard to retrieval of specific precautions on the Internet, there is an urgent need to train nurses to improve their online competence (Foundation of Nursing Studies, 2001). Several training programs have been reported to be effective, such as the "Usage of Online Information Resources by Nurses Project" (Wozar \& Worona, 2003). Such training programs should be part of the intervention to stimulate website adoption.

Furthermore, websites' functionalities should become more elegant in order to meet nurses' limited information-seeking skills. Ideally, websites communicating clinical practice guidelines should be equipped with a dialogue function that enables nurses to ask for clarification and follow-up questions. The design and evaluation of such technologies should be the subject of further research. 
The practical value of the website demonstrated in Chapters 4 and 5 is no guarantee of the uptake of the website in daily work practice. Several obstacles to the adoption of web-based guidelines remain. In order to identify a strategy to enhance adoption, barriers and facilitators of website adoption among 20 health care workers (HCWs) were studied.

In total, 20 semi-structured interviews were conducted with HCWs, the majority of whom had prior experience with the website. HCWs were asked to give their opinions about factors that hinder or facilitate adoption of the website in practice. The interviews were based on the PRECEDE model (Predisposing, Reinforcing, and Enabling Causes in Educational Diagnosis and Evaluation), complemented by quality criteria for health-related websites.

The results showed that the organization is a paramount reinforcer of website adoption among HCWs. Since HCWs value personal experience and communication with colleagues over online sources, it is of vital importance that a website addresses HCWs' particular needs and that the technology fits within the informal mechanisms of communication operative in HCWs' social networks. This can be realized by stimulating interpersonal (face-to-face) communication between HCWs from different occupational groups in both a formal (training) and informal (feedback) manner. The training should be provided by the most important opinion leaders in the organization; infection control nurses. In this way, personal resistance among physicians and infection control staff regarding the website may be eliminated.

This study reported in this chapter further shows that a user-driven design process does not ensure that potential adopters will be favorably predisposed toward the website. When organizational factors are not considered during the implementation process, adoption will probably fail. Furthermore, the study demonstrated that involvement of HCWs in the adoption process created a sense of awareness toward the website. This possibly fosters applicability of the website and leads to a willingness to integrate the website into daily practice. 


\section{Appendix}

Screenshots of the website 



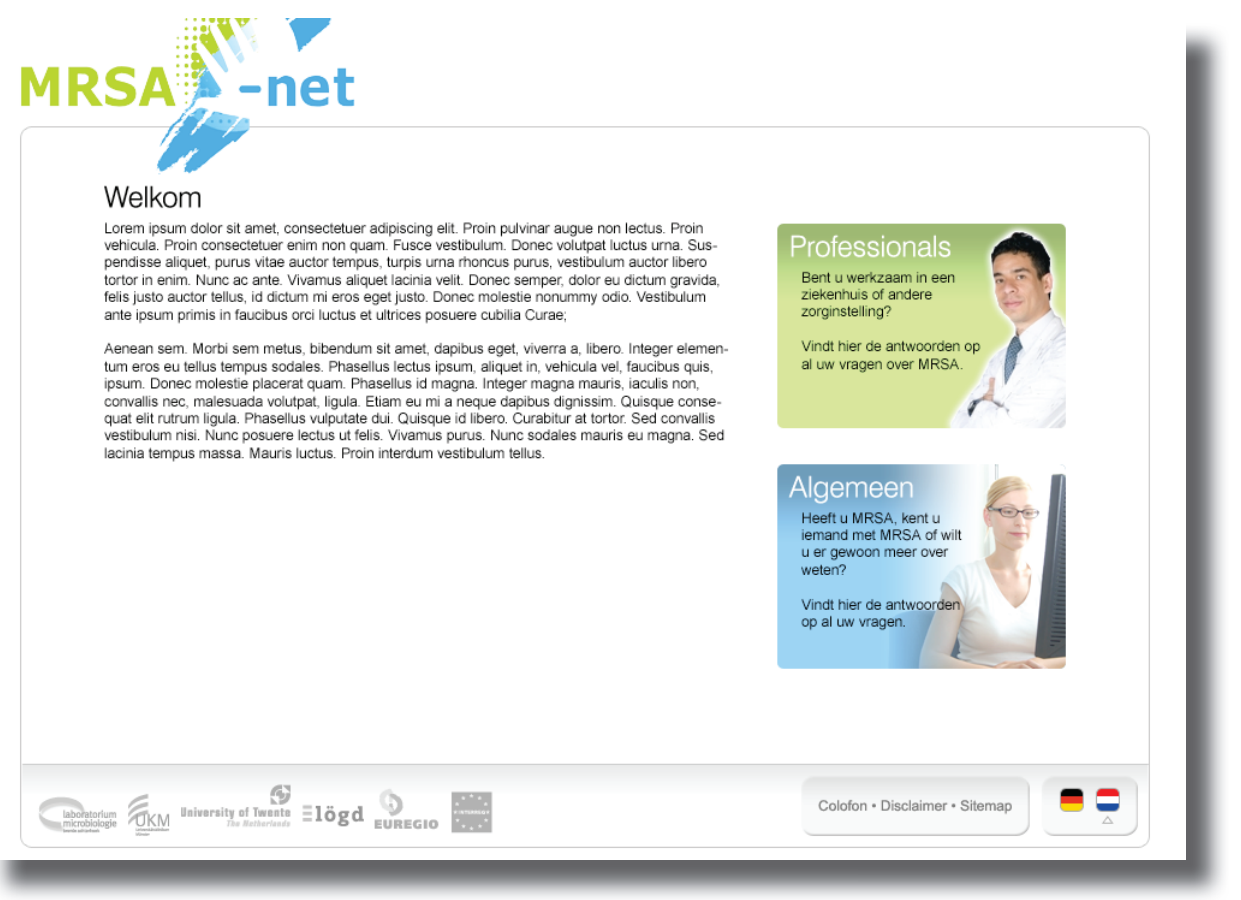

Figure 1. Screen mock-up prototype 1: Home

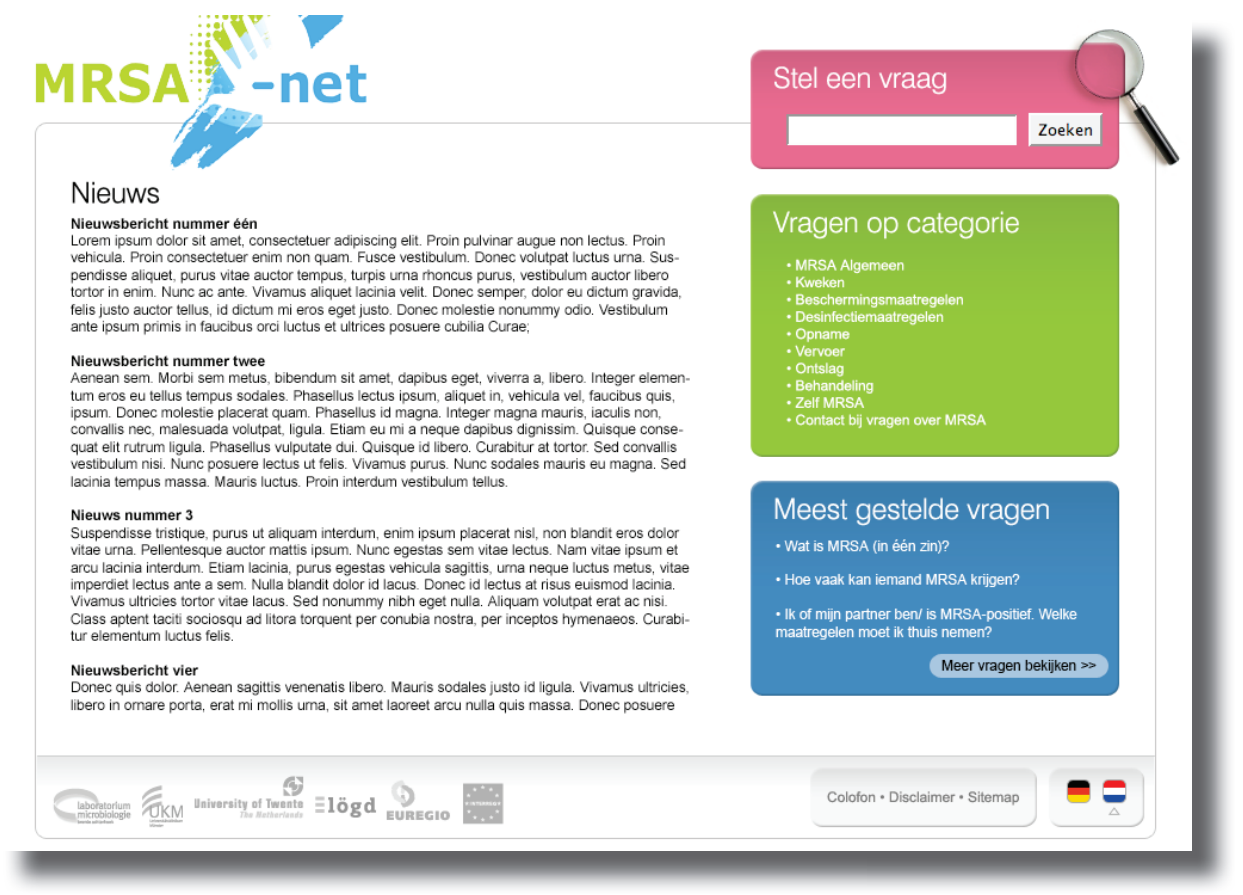

Figure 2. Screen mock-up prototype 1: Search page 


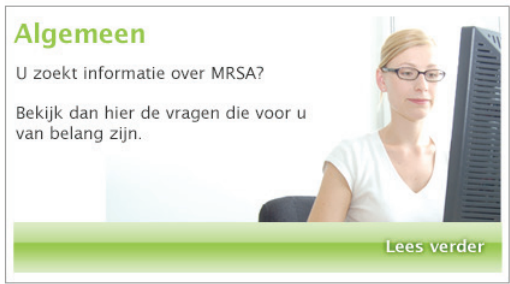

\section{Professionals}

Werkzaam in het ziekenhuis, of regel matig in aanraking met patienten?

Bekijk dan hier de vragen die voor u als professional van belang zijn.

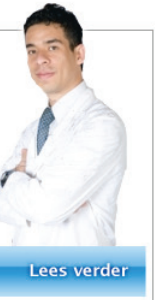

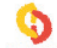
EUREGIO

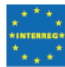

家M

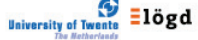

Figure 3. Screen mock-up prototype 2: Homepage

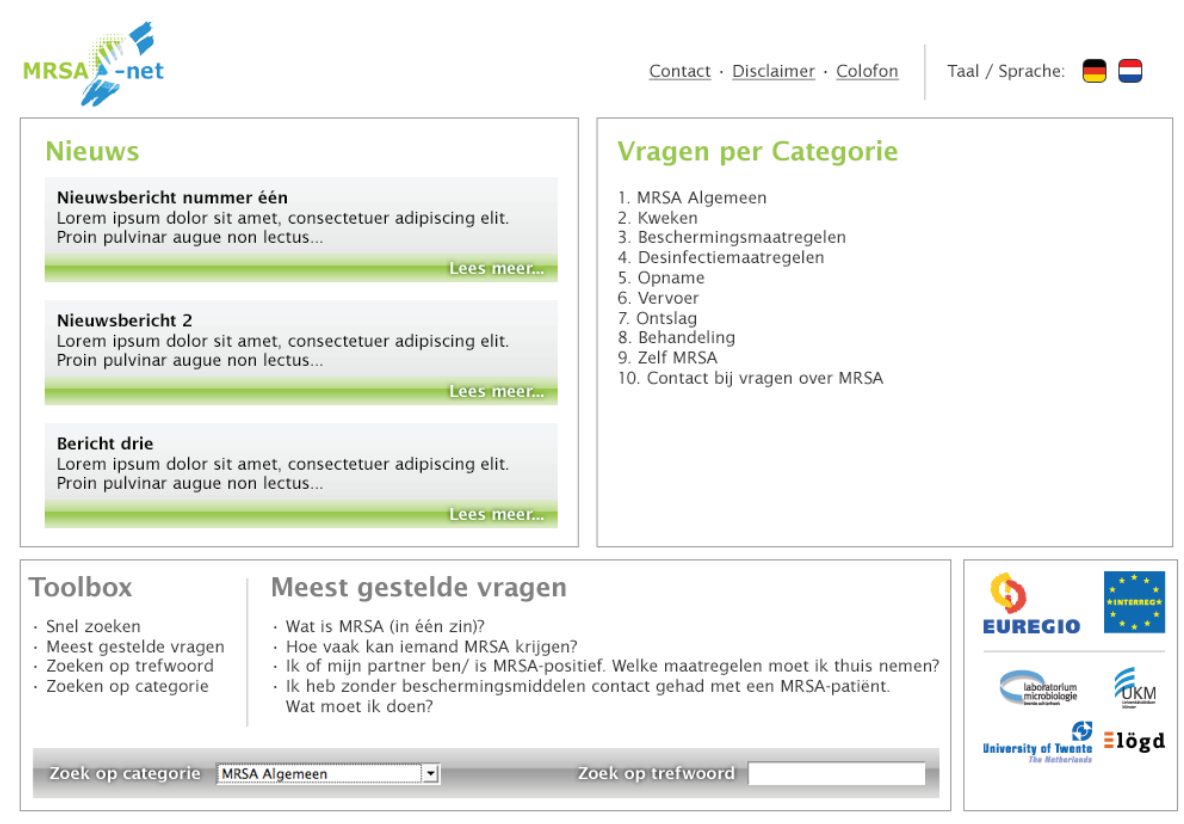

Figure 4. Screen mock-up prototype 2: Search page 


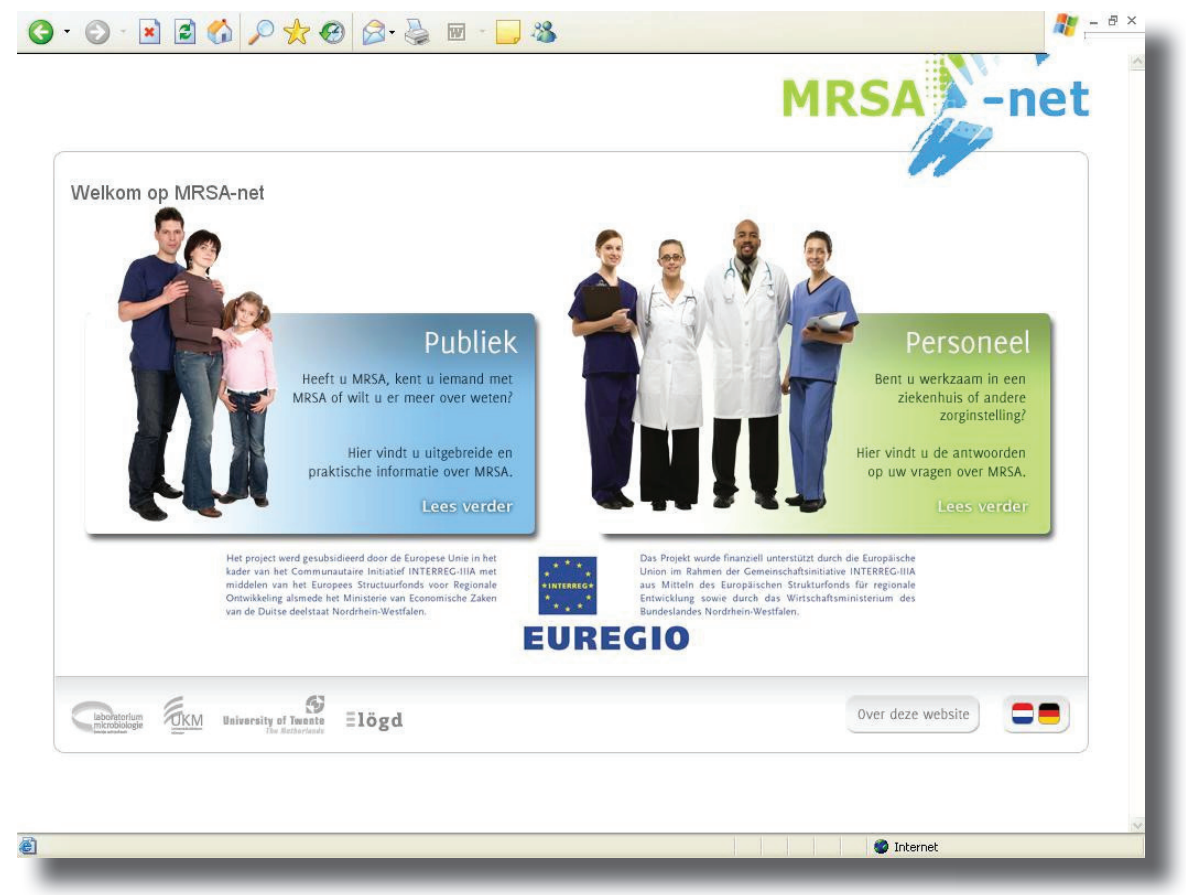

Figure 5. Final version of the website's homepage

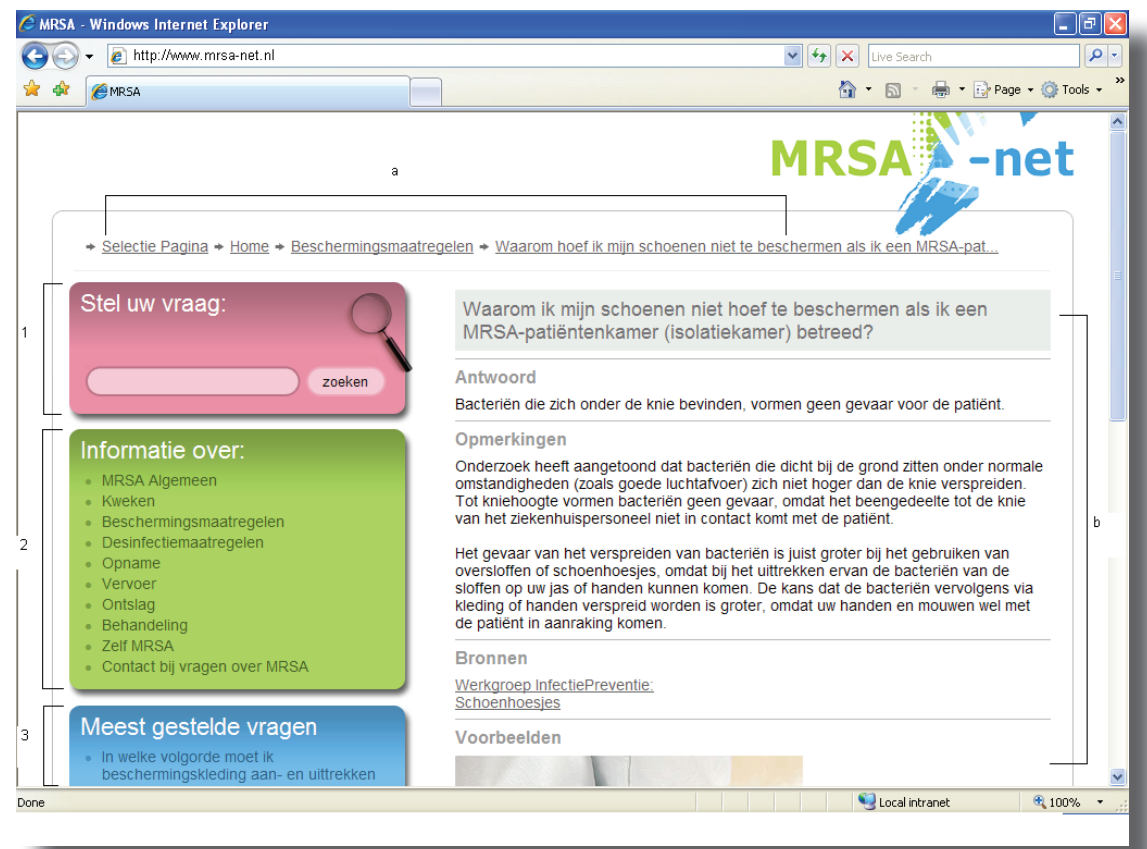

Figure 6. Final version of an answer page of the website

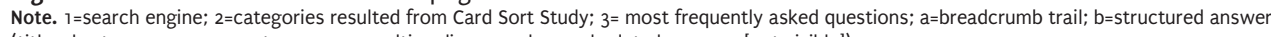
(title, short answer, comments, sources, multimedia examples, and related answers [not visible]) 


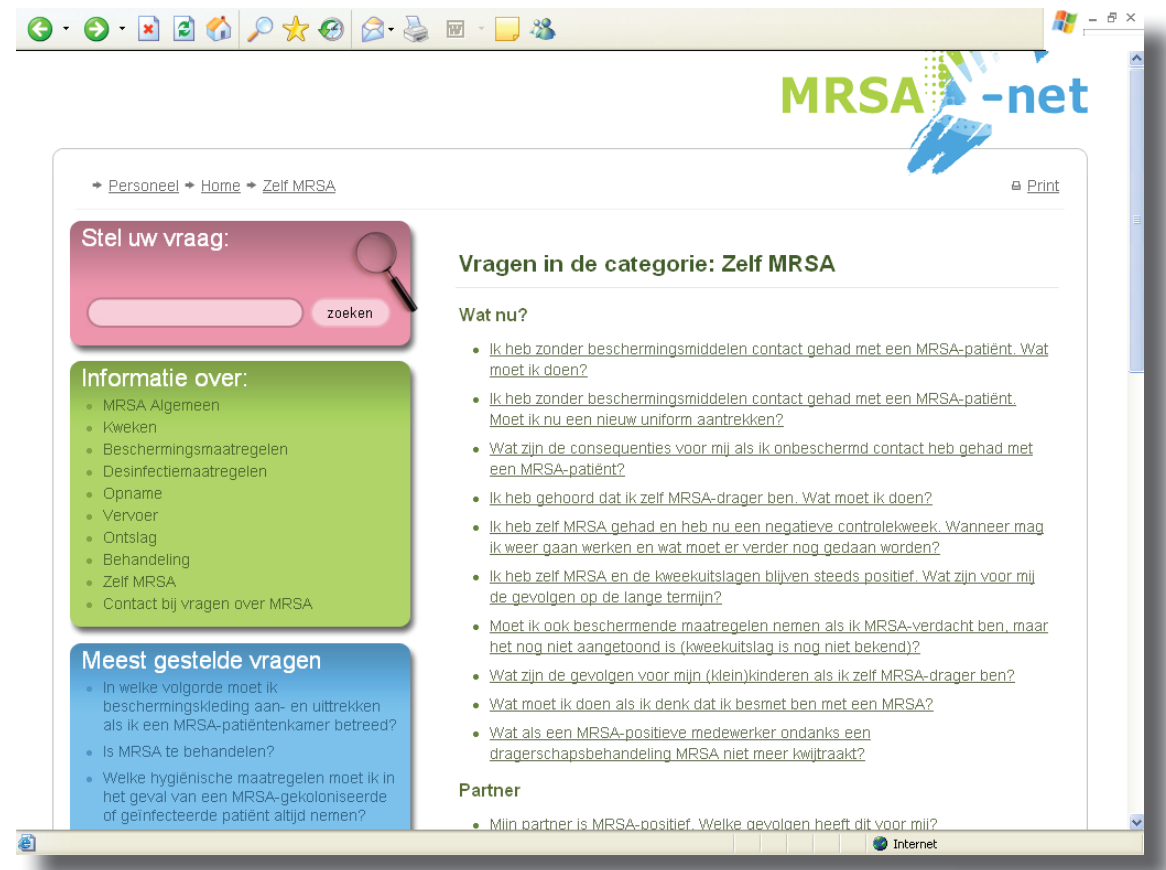

Figure 7. Screenshot of the categorical presentation of information on the website

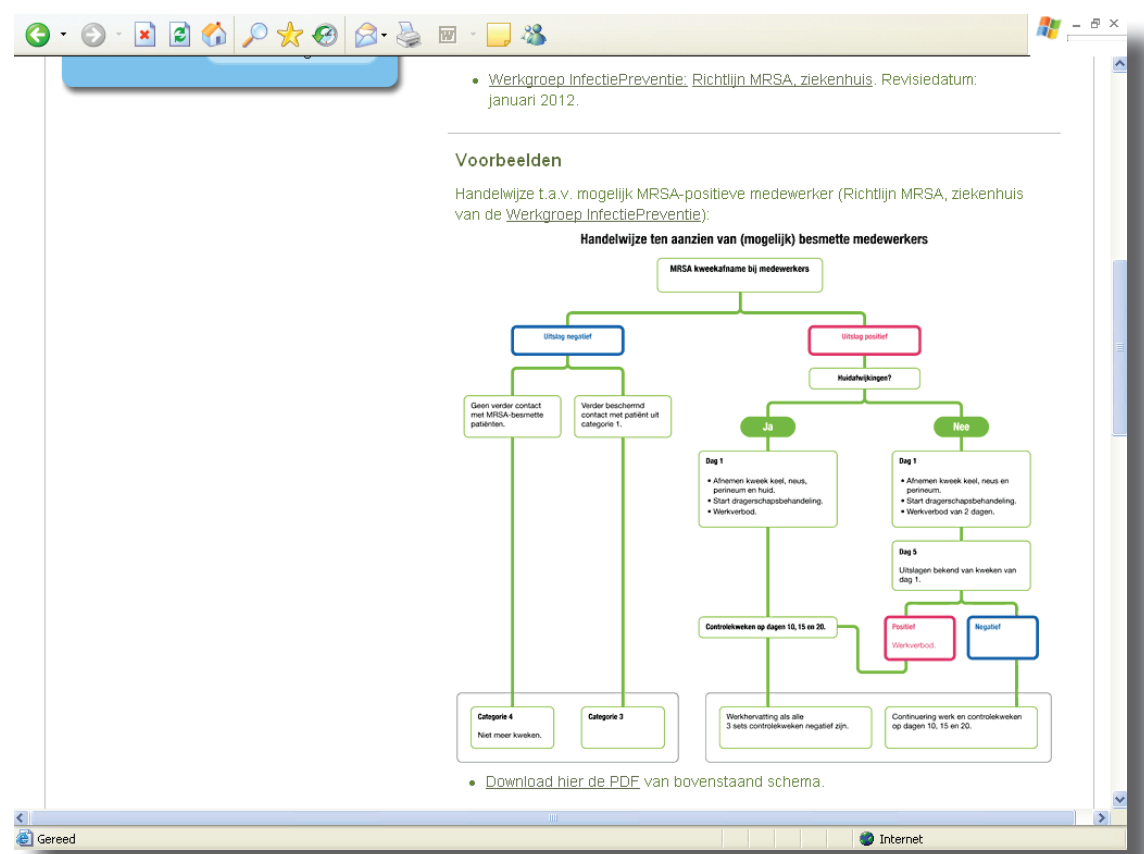

Figure 8. Screenshot of a tree diagram on the website 


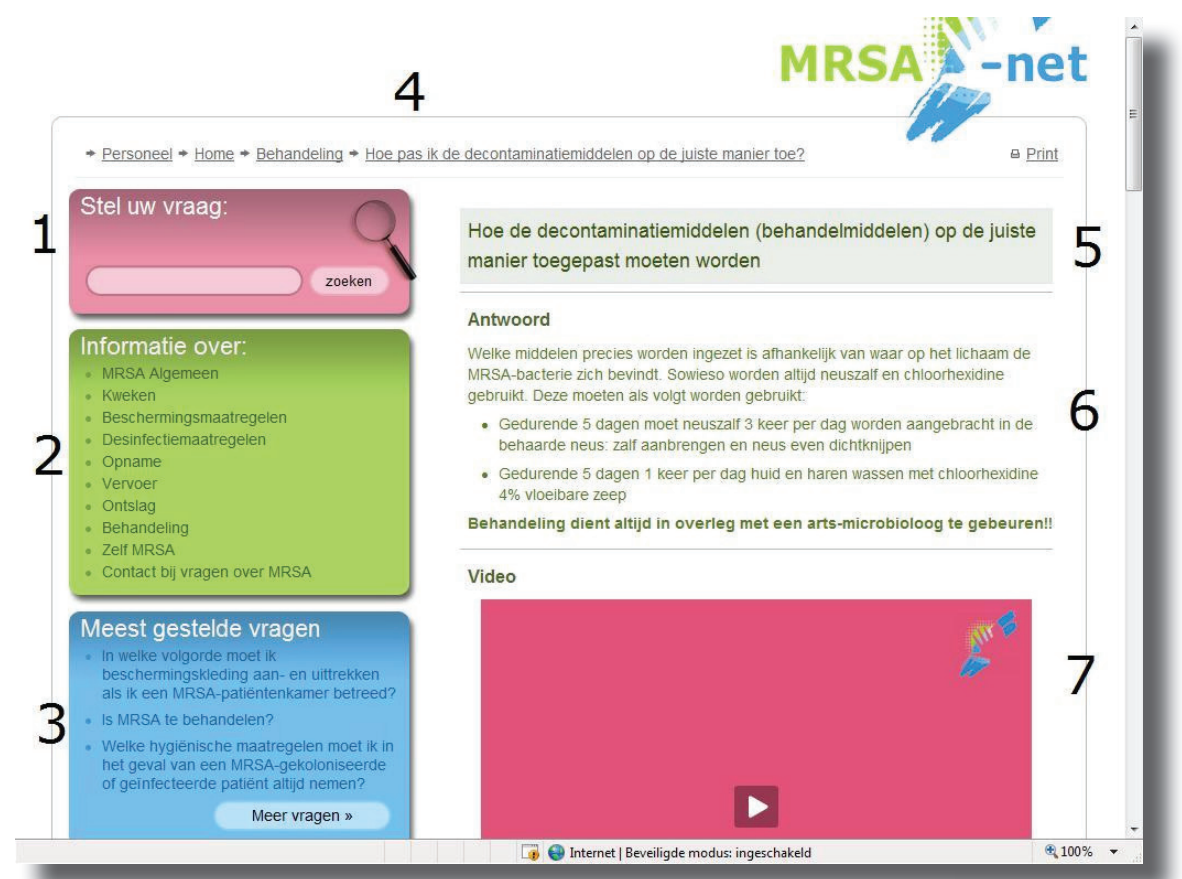

Figure 9. Screenshot of the website, showing the video screen

Note. $1=$ search engine; 2 = categories; 3 =frequently Asked Questions; $4=$ breadcrumb trail; 5 =answer title; $6=$ short answer; $7=$ video. 


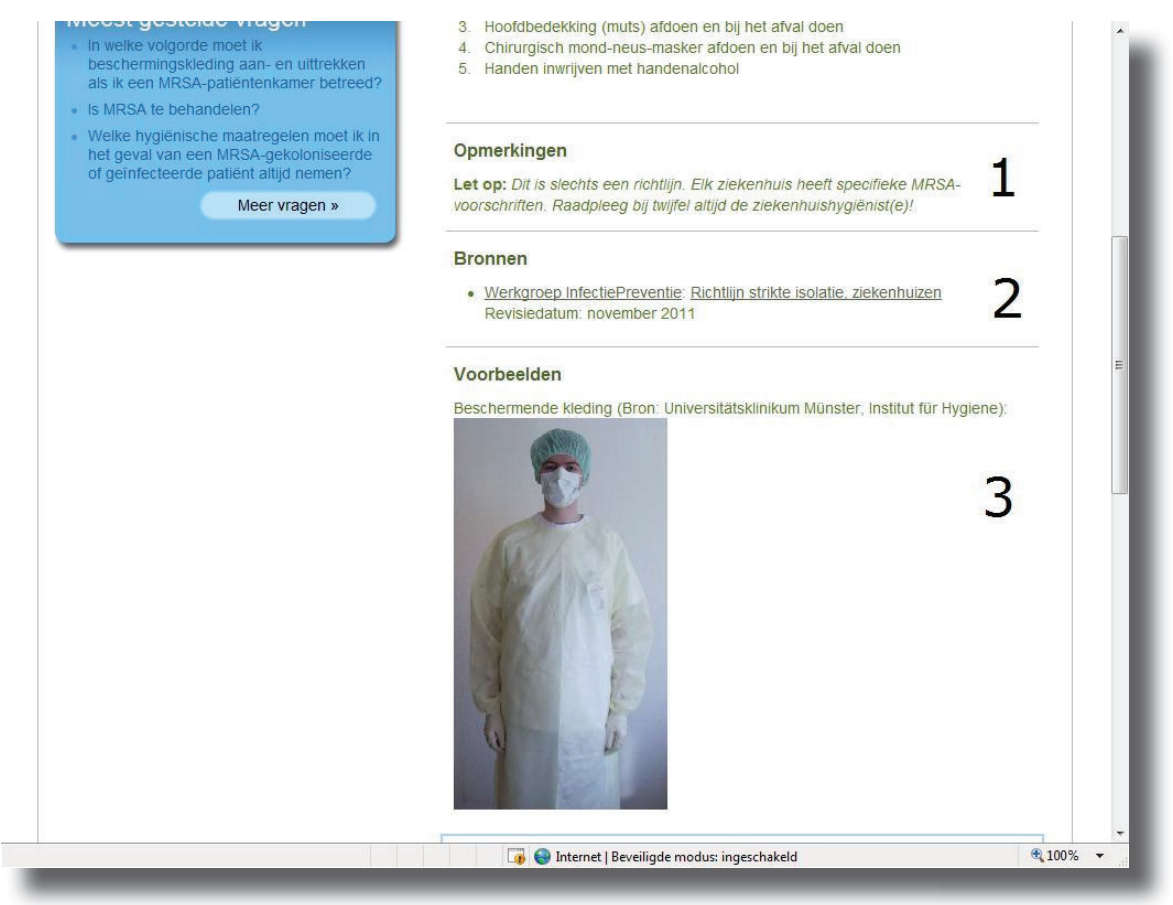

Figure 10. Screenshot of the website with an illustrative picture showing how to adequately wear protective clothing

Note. $1=$ comments; 2 =references; 3 =examples. 


\section{Chapter 6 \\ Factors affecting health care workers' adoption of a website with infection control guidelines}

Based on: Verhoeven F, Steehouder MF, Hendrix MGR, Gemert-Pijnen JEWC van. Factors affecting health care workers' adoption of a website with infection control guidelines. International Journal of Medical Informatics 2009. doi: 10.1016/j.jimedinf.2009.06.001 


\section{Chapter 2}

Assess and analyze needs

\section{Chapters $3 \& 4$}

Rank and select needs

\section{Chapters 3 \& 4}

Identify solutions,

Articulate goals

\section{Chapters $3 \& 4$}

Design and development

\section{Chapter 5}

Implement solution

\section{Chapter 6}

Users adopt solution

\section{Chapter 7}

Users realize goals 


\section{Factors affecting health care workers' adoption of a website with infection control guidelines}

\subsection{Introduction}

Health care-associated infections cause thousands of preventable deaths each year (Pittet \& Donaldson, 2006). It is therefore crucial that health care workers (HCWs) adhere to infection control guidelines. Although most HCWs are aware of the rationale for infection control practices, adherence to guidelines is generally poor (Berhe et al., 2005). This is partly due to problems with the guidelines' usability (Van GemertPijnen et al., 2005). A possible solution to this problem is to communicate guidelines via a website. A website enables the use of hyperlinks to additional resources and the use of multimodal functionalities, such as instructional videos. Consequently, indepth information can be made available without affecting the guidelines' readability (Fervers et al., 2005). Moreover, web-based guideline communication has the potential to combine information on relevant activities by facilitating information-seeking and retrieval processes in order to provide high-quality and appropriate answers to specific clinical questions, which is the most common reason that HCWs use the Internet (Casebeer et al., 2002; Jeannot et al., 2003). Therefore, HCWs' adherence might be better to electronic guidelines than to paper guidelines (Thomas et al., 1999). Other reported benefits of web-based guideline communication are decreased practice variations between clinicians (Morris, 2000; Grimshaw \& Russell, 1993) and improved patient outcomes (Garg et al., 2005; Grimshaw \& Russell, 1993).

Despite the proven value of web-based guidelines, numerous obstacles to the adoption of web-based guidelines in daily work practice remain (Jeannot et al., 2003). In this chapter, adoption refers to the process by which individuals and groups decide to use a website for the retrieval of infection control guidelines. Adoption is a prerequisite for successful implementation of a website with infection control guidelines in health care settings (Cain \& Mittman, 2002). Previous studies found several factors that may hinder the adoption of web-based guidelines, including lack of a computer connection in the HCW's office and fear that using such guidelines might interfere with the direct patient-physician relationship. HCWs are also concerned about the relevance and accuracy of instructions and the inability of web-based guidelines to adapt to varied situations (Jeannot et al., 2003; Rousseau et al., 2003). Despite the identification of these adoption barriers, specific intervention strategies for effective implementation of website with infection control guidelines are not yet available. As systematic reviews have shown that intervention strategies taking into account individual, work-related, and organizational factors are most likely to be successful (Grol \& Wensing, 2004), we investigated the factors affecting HCW' 
adoption of web-based guideline communication from a multi-faceted perspective.

Several models have been used to examine technological adoption, and all have their foundation in Rogers' classical Diffusion of Innovation Model. Examples include Social-Cognitive Theory, the Technology Acceptance Model, and the Unified Theory of Acceptance and Use of Technology (UTAUT) (Peters, 2007). Each of these models identifies three major groups of factors: individual factors (micro), such as a reluctance to change; work-related factors (meso) like sufficient staff and time; and organizational factors (macro) like support from peers or superiors (Dejoy et al., 2000; Francke et al., 2008; Gershon et al., 2000; Lundstrom et al., 2002; McGovern et al., 2000). Most models assign equal importance to the influence of each of these three groups of factors on adoption. However, considering that successful adoption of a new technology in health care implies changes in the organization, technology, and people involved, it is important to examine such phenomena from a social interactionist perspective, which implies that the three aforementioned groups of factors mutually reinforce one another. For instance, efforts to motivate HCWs to adopt web-based guideline communication may fail if the ward's management is not supportive. The PRECEDE (Predisposing, Reinforcing, and Enabling Causes in Educational Diagnosis and Evaluation) model recognizes that factors at each of the three groups of factors are interrelated and each are situated on another hierarchical rank (Dejoy et al., 2000). While other models assume the three separate groups of factors only have direct effects on intended behavior, the PRECEDE model suggests that there is also an indirect effect of organizational factors (macro) like management values or interpersonal communication on behavior. Moreover, these effects are mediated by work-related (meso) and individual (micro) factors (see Figure 6.1.). The PRECEDE model has also been presented as an applicable framework for factors affecting technology adoption (Kukafka et al., 2003). Therefore, we deem the PRECEDE model as appropriate to studying barriers and facilitators of adopting a website with infection control guidelines among HCWs. 


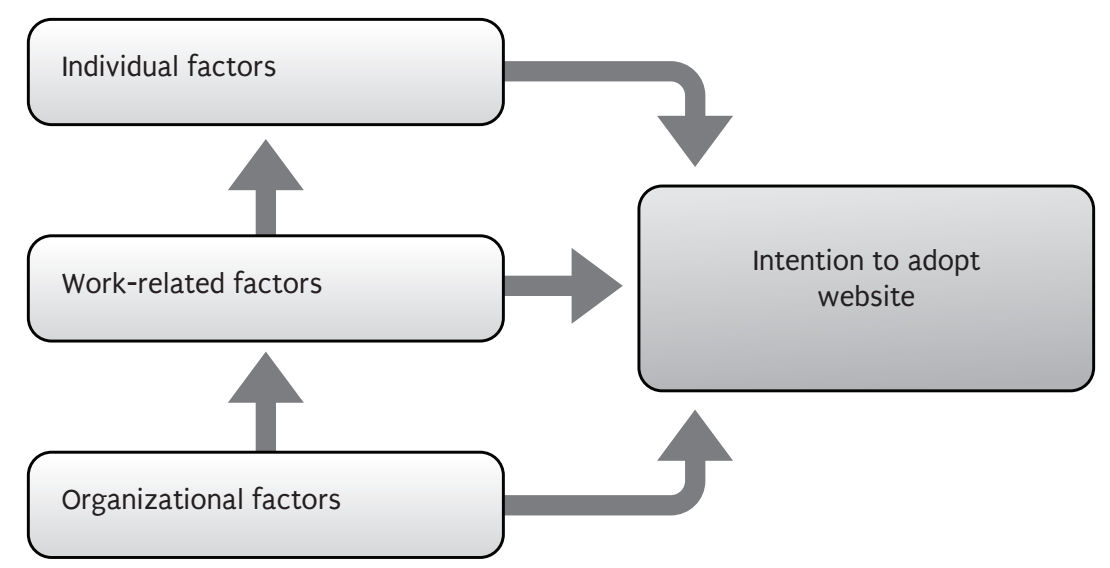

Figure 6.1. PRECEDE model

A fourth group of factors that is not considered by the PRECEDE model concerns the characteristics of the technology itself. Particularly when communicating clinical practice guidelines, it is of vital importance to ensure that the information presented is accurate, complete, and readable. Moreover, the website communicating the guidelines should be easy to use (Eysenbach et al., 2002; Kerr et al., 2006). Therefore, we complemented the PRECEDE model with a fourth group of factors comprising quality characteristics of health-related websites (Eysenbach et al., 2002) and used this as the conceptual framework for our study (see Table 6.1.).

The aim of this study was to identify any major factors and their interrelationship that could affect HCWs' adoption of a website for communicating infection control guidelines, and to use the findings to stimulate adoption of the website by HCWs in daily work practice.

\subsection{Methods}

\subsubsection{Research context}

Due to increased international trade in health services, Methicillin Resistant Staphylococcus aureus (MRSA) is one of the organisms that most commonly contributes to the rapid spread of health care-associated infections (Pittet \& Donaldson, 2006). The Dutch-German research project EUREGIO MRSA-net Twente/ Münsterland addresses this problem at a cross-national level, and the study reported here was conducted as part of this project (Friedrich et al., 2008). This study was conducted between April and June 2008.

This study focuses on the adoption of a website comprising MRSA guidelines. The website (www.mrsa-net.nl) was launched in February 2008 and was gradually developed following a specific user-centered design procedure (Kinzie et al., 2002). First, 28 scenario-based tests were conducted during which HCWs used paper-based guidelines to solve MRSA-related questions. The detected problems gave input to 
Table 6.1. Conceptual framework to identify barriers and facilitators of health care workers' adoption of a website with infection control guidelines

\begin{tabular}{|c|c|c|}
\hline Category & Factors & Description \\
\hline \multirow[t]{6}{*}{ Website quality } & Accuracy & $\begin{array}{l}\text { Degree to which information provided by the website is in concurrence with the } \\
\text { best available evidence, as perceived by the individual }\end{array}$ \\
\hline & Completeness & $\begin{array}{l}\text { Degree to which information provided by the website includes necessary parts, } \\
\text { elements, or steps to make a decision in daily practice, as perceived by the } \\
\text { individual }\end{array}$ \\
\hline & Readability & $\begin{array}{l}\text { Degree of complexity, and lengthiness of sentences of the provided information } \\
\text { on the website, as perceived by the individual }\end{array}$ \\
\hline & Design & Visual aspects of the website layout, as perceived by the individual \\
\hline & References provided & $\begin{array}{l}\text { Degree to which the individual is referred to other information sources on the } \\
\text { website, as perceived by the individual }\end{array}$ \\
\hline & Disclosures & $\begin{array}{l}\text { Degree to which information about terms of use, copyright, links to other sites, } \\
\text { and liability is provided by the website, as perceived by the individual }\end{array}$ \\
\hline \multirow[t]{2}{*}{ Predisposing (individual) } & Attitude & $\begin{array}{l}\text { The value or benefit anticipated from the website relative to current practice, } \\
\text { as perceived by the individual }\end{array}$ \\
\hline & Personality & $\begin{array}{l}\text { The degree to which an individual health care worker shows initiative to try } \\
\text { new things such as the website }\end{array}$ \\
\hline \multirow[t]{4}{*}{ Enabling (work-related) } & Workload & $\begin{array}{l}\text { The degree to which the amount of work needed to be performed is feasible } \\
\text { within a specific period, and whether the website is compatible with this } \\
\text { amount of work }\end{array}$ \\
\hline & Tacit knowledge & $\begin{array}{l}\text { Knowledge that is context-dependent and made up of the practical and } \\
\text { experiential wisdom of individuals in concurrence or in contrast with guidelines } \\
\text { provided by the website }\end{array}$ \\
\hline & Infrastructure & $\begin{array}{l}\text { The presence of some form of infrastructure (computers, internet connection) } \\
\text { or of other technologies in the ward, which cluster with the website }\end{array}$ \\
\hline & Work experience & $\begin{array}{l}\text { Degree to which the individual has become experienced with various technolo- } \\
\text { gies (such that the website can more easily coexist in daily work practice) }\end{array}$ \\
\hline \multirow[t]{5}{*}{$\begin{array}{l}\text { Reinforcing (organiza- } \\
\text { tional) }\end{array}$} & Management values & $\begin{array}{l}\text { Degree to which the opinion of an individual is respected or listened to by oth- } \\
\text { ers in terms of the adoption of the website }\end{array}$ \\
\hline & Training/ feedback & $\begin{array}{l}\text { The degree to which individuals obtain the opportunity to learn and discuss } \\
\text { how and why to apply the website }\end{array}$ \\
\hline & Communication channels & $\begin{array}{l}\text { The paths through which opinion leaders and others communicate about the } \\
\text { website (benefits and drawbacks) }\end{array}$ \\
\hline & Subjective norm & $\begin{array}{l}\text { Rules, formal hierarchies, and informal mechanisms of communication operative } \\
\text { in the social system (the ward) that might influence individual health care work- } \\
\text { ers' adoption of the website }\end{array}$ \\
\hline & Behavioral intention & The individual health care worker's readiness to adopt the website \\
\hline
\end{tabular}


the website's structure and content. Second, a Card Sort Task $(n=10)$ generated the website's menu structure and prototyping interviews $(n=14)$ led to the website's final lay-out. Based on the results, the first working prototype was developed and tested formatively by conducting scenario-based tests again $(n=28)$ before the website's launch. In each phase of this user-centered design process, the sample included physicians, nurses, and nursing assistants. Before the website's official launch, a workshop was held among infection control professionals and other stakeholders to gather their opinion. During this workshop, a working prototype of the website was demonstrated by the researcher (FV). The goal of this workshop was for the researcher to learn from the infection control professionals' feelings and attitudes about the website, and to generate a sense of involvement among the infection control professionals toward the website. The workshop generated several crucial issues for improvement. For instance, the infection control professionals suggested that the website's objective should be clearly present on the website. Therefore, we added a button "About this website". Also, infection control professionals argued that we needed to add more pictures and videos. All relevant topics that were raised during the workshop were solved before the website was officially launched online.

The bilingual website presents current, national MRSA guidelines that are recognized as standards of care in their country (Dutch Working party on Infection Prevention, 2005; The Committee for Hospital Hygiene and Infection Prevention at the Robert Koch Institute, 2002) presented as answers to questions that reflect HCWs' practical informational needs, as defined in the first scenario-based study (see Table 6.2. for two examples) (see Chapter 4). Each answer comprises a title, a short answer, an instructional video, comments, references (e.g., newspaper articles, links to other websites) for further reading, and (multi-media) examples. Three search options (a search engine, ten categories representing over 160 questions, and a list of frequently asked questions) and a breadcrumb trail facilitate HCWs to find relevant information on the website. Figures 9 and 10 in the middle of this thesis depict a screen capture of the website, illustrating the search options and the answer structure. Since its launch, the website has been consulted daily by approximately 450 unique visitors. Although the questions on both the Dutch and German part of the website are identical, the answers have to be country-specific due to differences in national MRSA-policy. E.g., in the Netherlands, surveillance cultures are taken upon hospital admission from people who are in close contact with living pigs and calves, in contrast to Germany. ${ }^{\text {' }}$ Therefore, answers were based on the country's national MRSA guidelines and complemented and validated by a medical microbiologist from the concerned country.

No particular accreditation system for health-related websites, such as the Health on

\footnotetext{
' Although the MRSA prevention and control policies across countries worldwide are comparable on the whole (e.g., see C.A. Muto, J.A. Jernigan, B.E. Ostrowsky, H.M. Richet, W.R. Jarvis, J.M. Boyce, et al. SHEA guidelines for preventing nosocomial transmission of multidrug-resistant strains of Staphylococcus aureus and Enterococcus. Infect. Control Hosp. Epidemiol. 24 (5) (2003) 362-386.), there are subtle differences among countries. Particularly in the Netherlands, the MRSA policy has a specific "search and destroy" character, comprising rigid isolation and screening of high-risk patient groups, screening low-risk groups, strict isolation of carriers, and treatment of people carrying MRSA. This "search and destroy" is not enforced in Germany. Therefore, the answers on the German and Dutch part of the website could not be formulated identically.
} 
the Net (HON)-code, has yet been successfully determined and implemented in the Netherlands and Germany. To assure credibility of the website's content, we used the national MRSA guidelines that are considered as professional standards of care and are used as such by the Public Health Inspector in both Germany and the Netherlands (Dutch Working party on Infection Prevention, 2005; The Committee for Hospital Hygiene and Infection Prevention at the Robert Koch Institute, 2002). We applied the basic quality criteria for guideline communication in order to meet the guidelines' "regulation function", as defined by the International Organization for Standardization (McRobb, 1990). In order to meet the "communication function", we applied researchbased web design and usability guidelines provided by the U.S. Department of Health and Human Services (Koyani et al., 2006) and results from a content analysis of existing MRSA guidelines (see Chapter 2).

\subsubsection{Sample}

Respondents $(\mathrm{N}=20)$ were employees of hospitals in the Euregio Twente/Münsterland, and represented the website's main target groups (nursing assistants, nurses, and physicians), and relevant stakeholders (infection control nurses and medical microbiologists). The sample comprised four representatives from each target group. Respondents were selected from a list provided by the infection control nurses at all hospitals in the Euregio Twente/Münsterland. Ultimately, the study was conducted in two Dutch hospitals (bed sizes 314 and 1070) and two German hospitals (bed sizes 405 and 1500). We selected these hospitals because together they represented several hospital categories (urban, rural, teaching, and non-teaching). From each country, a rural non-teaching hospital was represented next to an urban teaching hospital. Besides, these hospitals were selected because of their location in the Dutch-German border region, where the research project EUREGIO MRSA-net Twente/Münsterland was carried out (see section 6.2.1.). Respondents had used the website before, and their mother tongue was either Dutch or German. Eight of the respondents were male, twelve were female. On average the respondents had been working in health care for 17.7 years (range $0.25-25$ ), and in their current job for 7.6 years. The majority $(n=16)$ were full-time employees in their current job position.

\subsubsection{Measures}

An interview guide was constructed, based on the aforementioned conceptual framework (see Table 6.1.1.). Open-ended questions were formulated in order to probe HCWs about their perceptions of and experiences with the website (see Table 6.3.). Since we were primarily interested in exploring the practical meaning HCWs gave to theoretical concepts in order to tailor adoption-enhancing intervention strategies to HCWs' perception of the environment, rather than testing a number of propositions, we opted to conduct interviews with open-ended questions (Van Velsen et al., 2008). The questions represented the factors inherent in the PRECEDE model (attitude, personality, workload, tacit knowledge, infrastructure, work experience, management values, training and feedback, communication channels, and subjective norms), in addition to questions about website quality and intentions to adopt the website in 
Table 6.2. Two examples of question-answer pairs that can be found on the website with Methicillin Resistant Staphylococcus aureus guidelines

\begin{tabular}{|c|c|c|}
\hline & Example 1 & Example 2 \\
\hline Question & $\begin{array}{l}\text { In which order do I have to put on and off protective } \\
\text { clothing before entering an isolation room? }\end{array}$ & $\begin{array}{l}\text { Is it dangerous to nurse MRSA-positive patients when I } \\
\text { am pregnant? }\end{array}$ \\
\hline \multirow[t]{10}{*}{ Short answer } & $\begin{array}{l}\text { Before entering the room, put on the protective clothing } \\
\text { in the anteroom in this order: } \\
\text { 1. Put on gloves }\end{array}$ & $\begin{array}{l}\text { Basically, the nursing of an MRSA-positive patient does } \\
\text { not imply any personal risk for you or your baby, so you } \\
\text { can nurse an MRSA-positive patient without running any } \\
\text { occupational hazard during your pregnancy. }\end{array}$ \\
\hline & 2. Put on long-sleeved gown & \\
\hline & 3. Put on mask & \\
\hline & 4. Put on hat & \\
\hline & $\begin{array}{l}\text { When leaving the room, take off protective clothing in } \\
\text { the following order: }\end{array}$ & \\
\hline & $\begin{array}{l}\text { 1. In the isolation room: Take off gloves and decontami- } \\
\text { nate hands with an antiseptic-containing preparation }\end{array}$ & \\
\hline & $\begin{array}{l}\text { 2. In the anteroom: Take off long-sleeved gown and fold } \\
\text { it inside out before throwing it into the dustbin, or in } \\
\text { case of reuse, hang gown up inside out on the coathooks }\end{array}$ & \\
\hline & 3. Take off hat and throw it into the dustbin & \\
\hline & 4. Put off mask and throw it into the dustbin & \\
\hline & $\begin{array}{l}\text { 5. Decontaminate hands again with antiseptic-containing } \\
\text { preparation }\end{array}$ & \\
\hline Comments & $\begin{array}{l}\text { Please note: This is only a guideline. Each hospital has } \\
\text { its own specific MRSA-precautions. In case of any doubt, } \\
\text { please consult your hospital's infection control profes- } \\
\text { sional! }\end{array}$ & $\begin{array}{l}\text { Actually, you are nowhere better protected against } \\
\text { MRSA than in the patient room. If you put on and off the } \\
\text { protective clothing adequately, and adhere to hygiene } \\
\text { guidelines regarding hands, equipment, and surfaces, } \\
\text { nothing will happen. }\end{array}$ \\
\hline
\end{tabular}


practice. The questions were derived from several existing, validated instruments fitting the study's themes (Dejoy et al., 2000; Gershon et al., 2000; Lundstrom et al., 2002; McGovern et al., 2000). The face validity of the interview guide was tested by piloting it face-to-face with one Dutch and one German medical microbiologist involved in the research project (not included in the sample). Also, we determined inter-rater reliability during data analysis to verify whether the questions were actually appropriate for measuring the factors from our conceptual framework.

\subsubsection{Procedure}

The interviews were conducted by an interviewer who spoke fluent Dutch and German. The interviewer brought a laptop with an offline version of the website to the interview location in case the respondent needed a reminder of the website. The purpose and procedure of the interview were described in general terms at the beginning of each interview. It was emphasized that the respondents' contribution would be strictly confidential. Then the interview began with the interviewer following the interview scheme as presented in Table 6.3. The interview ended when the interviewer perceived that the factors of the conceptual framework were exhausted and no new information could be expected. The interviews lasted between 45 and 75 minutes. The interviews were audiotaped with prior consent of all respondents and transcribed verbatim afterwards.

The study was approved by the management of the hospitals in which the respondents were employed. Respondents gave oral consent to participate and for recording the interviews with a digital voice recorder. We emphasized that the respondents' contribution would be strictly confidential and that data would be immediately destroyed after data transcription.

\subsubsection{Analysis}

Data from one randomly selected transcribed interview were analyzed by two coders (FV, JvG) using deductive analysis, implying that the coding categories were derived from the conceptual framework presented in Table 6.1. (Patton, 1990). Four new factors emerged during the coding process (relevance, findability, usability, and target group) that could not be assigned to one of the categories from the framework. Therefore, these factors were added to the coding scheme. Subsequently, all transcribed data were coded by means of the revised framework. Repeated or reworded descriptions of the same factors were counted only once per interview.

Overall, 361 statements about website adoption were identified and coded by a single coder (FV). Then another researcher (JvG) coded a random selection of $10 \%$ of all coded fragments. Both (Dutch) researchers had an excellent command of the German language. Therefore, Dutch and German data were analyzed in their original wordings without translating all data into a similar language. Agreement on categorization of problems and requirements was "almost perfect" (Cohen's Kappa $=0.82$ ) (Landis \& Koch, 1977). All data were analyzed by hand, without the use of qualitative data analysis software. Although we are aware of the benefits that data analysis software 
Table 6.3. Interview guide with open-ended questions to identify barriers and facilitators of health care workers' adoption of a website with infection control guidelines

\begin{tabular}{|c|c|c|}
\hline Category & Factor & Factors and questions \\
\hline \multirow[t]{7}{*}{ Website quality } & Website quality & 1. \\
\hline & & $\begin{array}{l}\text { Do you think that the information on the website is in concurrence with } \\
\text { the best evidence? (accuracy) }\end{array}$ \\
\hline & & $\begin{array}{l}\text { Do you think that the information provided by the website contains the } \\
\text { necessary parts to make a decision in daily practice? (completeness) }\end{array}$ \\
\hline & & $\begin{array}{l}\text { What do you think of the complexity and lengthiness of sentences of the } \\
\text { information on the website? (readability) }\end{array}$ \\
\hline & & - How would you rate the design of the website? (design) \\
\hline & & $\begin{array}{l}\text { What do you think of the references to other information sources on the } \\
\text { website? (references provided) }\end{array}$ \\
\hline & & $\begin{array}{l}\text { To what degree do you perceive that information about terms of use, } \\
\text { copyrights, and liability is provided on the website? (disclosures) }\end{array}$ \\
\hline \multirow[t]{2}{*}{ Predisposing (individual) } & Attitude & $\begin{array}{l}\text { 2. Do you think the website would be useful for you in daily work practice, } \\
\text { or for other target groups? What possible benefits and threats do you } \\
\text { perceive? }\end{array}$ \\
\hline & Personality & $\begin{array}{l}\text { 3. Do you think personality characteristics (e.g., self-direction, conformity) } \\
\text { will affect use of the website in daily practice? }\end{array}$ \\
\hline \multirow[t]{4}{*}{ Enabling (work-related) } & Workload & 4. Do you believe workload will impede or stimulate use of the website? \\
\hline & Tacit knowledge & $\begin{array}{l}\text { 5. How would you rate your current knowledge level regarding MRSA guide- } \\
\text { lines, and to what degree will this influence use of the website? }\end{array}$ \\
\hline & Infrastructure & $\begin{array}{l}\text { 6. Do you think the presence of infrastructure (e.g., computers with Internet } \\
\text { connection) on your ward will affect website use? How? }\end{array}$ \\
\hline & Work experience & $\begin{array}{l}\text { 7. How would you rate your experience with using technology in your work } \\
\text { setting and do you expect this to hinder or facilitate website use? }\end{array}$ \\
\hline \multirow[t]{4}{*}{ Reinforcing (organizational) } & Management values & $\begin{array}{l}\text { 8. Does your ward employ any individuals whose opinions are respected or } \\
\text { even just listened to by others? Who are these individuals and how can they } \\
\text { possibly affect website use? }\end{array}$ \\
\hline & Training/ feedback & $\begin{array}{l}\text { 9. Is there any mechanism of frequent safety-related feedback or training } \\
\text { by co-workers or supervisors present on this ward? If yes, how would this } \\
\text { influence use of the website among health care workers? }\end{array}$ \\
\hline & Communication channels & $\begin{array}{l}\text { 10. Which paths do you perceive to be the most effective in communicating } \\
\text { to health care workers about the website? }\end{array}$ \\
\hline & Subjective norm & $\begin{array}{l}\text { 11. Does your colleagues' behavior normally influence your behavioral } \\
\text { intentions, and to what degree do you think this will affect your intention } \\
\text { to use the website? }\end{array}$ \\
\hline Behavioral intention & Behavioral intention & $\begin{array}{l}\text { 12. Do you intend to use the website in the near future in your daily work } \\
\text { practice? Why (not)? }\end{array}$ \\
\hline
\end{tabular}


for qualitative research might have, we opted to analyze the data by hand for two reasons. First, the bilingual character of the data set hampered the ease of applying data analysis software. Second, we feared that by using data analysis software, the volume and breadth of the categories would become overemphasized, at the cost of depth and meaning of the measured constructs (rich meanings would be turned down into meaningless numbers) (Weitzman, 1999).

After coding, (nearly) synonymous statements were combined into more general subthemes in order to create a better-organized overview of the results. The subthemes that emerged are presented in Table 6.4. in the Results section.

\subsection{Results}

Table 6.4. reflects the opinions of HCWs' about website adoption as they emerged from the interview statements. Only issues that were mentioned by at least three different respondents are included. This section will elaborate on the most frequently mentioned factors, separated into the group of factors of the conceptual framework.

\subsubsection{Statements related to website quality}

\section{Website's general appreciation}

Respondents were generally positive toward the quality of the website. Out of 72 statements, 60 were positive and 12 were negative. Positive statements mainly concerned the website's relevance $(n=10)$, design $(n=12)$, and usability $(n=15)$.

With regard to relevance, the website's practical approach to infection control guidelines was appreciated in particular $(n=10)$ :

"Questions from daily life, about which you generally do not think of. Answered in great detail."

(German infection control nurse; see Table 6.4, Category 16)

\section{Website's design and usability}

The website's design was perceived as good due to its visual attractiveness $(n=7)$ and convenient arrangement of information $(n=5)$. Respondents pointed positively to the website's usability, which was easy to use $(n=6)$, accessible for a broad target group $(n=4)$, and it enabled them to find relevant information quickly $(n=5)$ : 
Table 6.4. Barriers and facilitators for adoption of a website with infection control guidelines resulting from interview statements $(\mathrm{N}=2 \mathrm{O})$

\begin{tabular}{|c|c|c|c|}
\hline Category & Factors & $\#$ & Barriers and facilitators for website adoption* \\
\hline \multirow{16}{*}{$\begin{array}{l}\text { Website quality } \\
\text { (72 statements) }\end{array}$} & \multirow[t]{3}{*}{ Accuracy } & 1 & $\begin{array}{l}\text { + The guidelines provided by the website are in concordance with most actual guidelines } \\
\text { available }(n=3)\end{array}$ \\
\hline & & 2 & - The website does not mention the frequency with which guidelines are updated $(n=3)$ \\
\hline & & 3 & $\begin{array}{l}\text { - The guidelines provided by the website are not in concordance with the best available } \\
\text { evidence }(n=3)\end{array}$ \\
\hline & Completeness & 4 & $\begin{array}{l}\text { + The website provides all necessary parts, elements, or steps for making a decision about } \\
\text { practice regarding MRSA }(n=4)\end{array}$ \\
\hline & \multirow[t]{2}{*}{ Readability } & 5 & $\begin{array}{l}\text { + The guidelines on the website are described concisely (adequate word length and sentence } \\
\text { length) }(n=3)\end{array}$ \\
\hline & & 6 & + The guidelines on the website are described clearly (without complexity) $(n=3)$ \\
\hline & \multirow[t]{2}{*}{ Design } & 7 & + The website looks visually attractive (colors, pictures) $(n=7)$ \\
\hline & & 8 & + The information on the website is conveniently arranged $(n=5)$ \\
\hline & $\begin{array}{l}\text { References } \\
\text { provided }\end{array}$ & 9 & $\begin{array}{l}\text { + Each guideline on the website contains references to scientific information and other } \\
\text { sources, which enhances the website's credibility }(n=5)\end{array}$ \\
\hline & Disclosures & 10 & $\begin{array}{l}\text { - The website does not mention which organization(s) developed the website/ wrote the } \\
\text { guidelines }(n=3)\end{array}$ \\
\hline & \multirow[t]{3}{*}{ Usability } & 11 & + The website is easy to use $(n=6)$ \\
\hline & & 12 & + The website enables health care workers to find relevant information quickly $(n=5)$ \\
\hline & & 13 & + The website is highly accessible for a broader target group $(n=4)$ \\
\hline & \multirow[t]{2}{*}{ Findability } & 14 & + The website is among the top three hits in Google when entering "MRSA" $(n=5)$ \\
\hline & & 15 & $\begin{array}{l}\text { - There is a similar website about the same topic with almost an identical URL (www.mrsa- } \\
\text { net.org instead of www.mrsa-net.nl), which is confusing }(n=3)\end{array}$ \\
\hline & Relevance & 16 & $\begin{array}{l}+ \text { The website presents practical questions that are of major interest to health care workers } \\
(n=10)\end{array}$ \\
\hline $\begin{array}{l}\text { Predisposing } \\
\text { (individual) }\end{array}$ & \multirow[t]{8}{*}{ Attitude } & 17 & $\begin{array}{l}\text { + The website enables health care workers to look up practical information at any time and } \\
\text { location }(n=5)\end{array}$ \\
\hline \multirow[t]{12}{*}{ (85 statements) } & & 18 & $\begin{array}{l}+ \text { The website is the first that handles the topic in such a profound manner, which enhances } \\
\text { credibility }(n=7)\end{array}$ \\
\hline & & 19 & + The website is valuable, with practical contributions to existing local guidelines $(n=4)$ \\
\hline & & 20 & $\begin{array}{l}\text { - Personal lack of interest in website content as it pertains to infection control guidelines } \\
(n=4)\end{array}$ \\
\hline & & 21 & - Personal resistance against Internet-related technologies $(n=5)$ \\
\hline & & 22 & $\begin{array}{l}\text { - The website is only of relevance for those who must actually adhere to the guidelines, such } \\
\text { as nursing staff }(n=3)\end{array}$ \\
\hline & & 23 & $\begin{array}{l}\text { - The guidelines communicated on the website are not in concordance with locally enforced } \\
\text { guidelines }(n=10)\end{array}$ \\
\hline & & 24 & $\begin{array}{l}\text { - The website cannot replace face-to-face contact; it can only serve as an additional com- } \\
\text { munication channel }(n=5)\end{array}$ \\
\hline & Personality & 25 & $\begin{array}{l}\text { + Self-direction is an important attribute that predisposes individual health care workers to } \\
\text { try out new things, such as the website }(n=4)\end{array}$ \\
\hline & \multirow[t]{4}{*}{ Target group } & 26 & + The website is useful for nurses $(n=12)$, \\
\hline & & 27 & + The website is useful for patients $(n=14)$ \\
\hline & & 28 & + The website is useful for patients' relatives $(n=6)$ \\
\hline & & 29 & + The website is useful for physicians $(n=6)$ \\
\hline
\end{tabular}

Continued 
Table 6.4. (continued) Barriers and facilitators for adoption of a website with infection control guidelines resulting from interview statements $(\mathrm{N}=20)$

\begin{tabular}{|c|c|c|c|}
\hline Category & Factors & $\#$ & Barriers and facilitators for website adoption* \\
\hline $\begin{array}{l}\text { Enabling (work- } \\
\text { related) }\end{array}$ & Workload & 30 & $\begin{array}{l}+ \text { The website lowers work pressure for infection control professionals, as they will receive } \\
\text { fewer questions from health care workers }(n=3)\end{array}$ \\
\hline \multirow[t]{6}{*}{ (53 statements) } & & 31 & - High work pressure impedes consulting the website during working hours ( $n=9$ ) \\
\hline & $\begin{array}{l}\text { Tacit knowl- } \\
\text { edge }\end{array}$ & 32 & $\begin{array}{l}\text { + The website enables health care workers with a lack of knowledge of MRSA guidelines to } \\
\text { retrieve relevant information }(n=4)\end{array}$ \\
\hline & & 33 & $\begin{array}{l}\text { - Health care workers do not need the website due to personal, practical, and experiential } \\
\text { wisdom about MRSA guidelines }(n=10)\end{array}$ \\
\hline & Infrastructure & 34 & $\begin{array}{l}\text { - The absence of computers with Internet connections in the work environment impedes } \\
\text { health care workers from consulting the website during working hours }(n=7)\end{array}$ \\
\hline & $\begin{array}{l}\text { Work experi- } \\
\text { ence }\end{array}$ & 35 & $\begin{array}{l}\text { + The website easily fits into daily work practices since health care workers have become } \\
\text { experienced with technology }(n=17)\end{array}$ \\
\hline & & 36 & $\begin{array}{l}\text { - If there is no immediate cause for website use during work practice, health care workers } \\
\text { will forget about the website's existence }(n=3)\end{array}$ \\
\hline $\begin{array}{l}\text { Reinforcing (or- } \\
\text { ganizational) }\end{array}$ & $\begin{array}{l}\text { Management } \\
\text { values }\end{array}$ & 37 & $\begin{array}{l}+ \text { Infection control nurses are the most important and adequate opinion leaders to advocate } \\
\text { website adoption }(n=10)\end{array}$ \\
\hline \multirow[t]{16}{*}{ (131 statements) } & & 38 & $\begin{array}{l}+ \text { Ward management is the most important and adequate opinion leader in general, including } \\
\text { for website adoption }(n=5)\end{array}$ \\
\hline & & 39 & $\begin{array}{l}\text { - Opinion leaders will not influence the behavioral intentions of individual health care workers } \\
\text { to use the website, as health care workers are self-directive }(n=8)\end{array}$ \\
\hline & & 40 & $\begin{array}{l}\text { - Infection control nurses are not effective opinion leaders to promote website use, as they } \\
\text { are not respected or listened to (they always "bring bad news") }(n=3)\end{array}$ \\
\hline & $\begin{array}{l}\text { Training/ } \\
\text { feedback }\end{array}$ & 41 & $\begin{array}{l}\text { + Obligatory training sessions are the best way to bring the website to health care workers' } \\
\text { attention }(n=13)\end{array}$ \\
\hline & & 42 & $\begin{array}{l}\text { + Enabling health care workers to actually use the website during obligatory training sessions } \\
\text { is the best way to predispose them favorably }(n=6)\end{array}$ \\
\hline & & 43 & $\begin{array}{l}\text { - Training sessions solely focus around the website, which health care workers have to attend } \\
\text { on a voluntary basis, will have no effect }(n=3)\end{array}$ \\
\hline & $\begin{array}{l}\text { Communica- } \\
\text { tion channels }\end{array}$ & 44 & $\begin{array}{l}\text { + Mutual recommendation of the website among health care workers is the best way to } \\
\text { stimulate adoption }(n=12)\end{array}$ \\
\hline & & 45 & $\begin{array}{l}\text { + A leaflet is an appropriate communication channel to promote website use among health } \\
\text { care workers }(n=10)\end{array}$ \\
\hline & & 46 & $\begin{array}{l}\text { + A team meeting is an appropriate communication channel to promote website use among } \\
\text { health care workers }(n=10)\end{array}$ \\
\hline & & 47 & $\begin{array}{l}\text { + A personal approach to health care workers by the website project team is an appropriate } \\
\text { communication channel to promote website use among health care workers }(n=6)\end{array}$ \\
\hline & & 48 & $\begin{array}{l}\text { + A link in existing, locally enforced guidelines is an appropriate communication channel to } \\
\text { promote website use among health care workers }(n=5)\end{array}$ \\
\hline & & 49 & $\begin{array}{l}+ \text { An e-mail to all health care workers is an appropriate communication channel to promote } \\
\text { website use among health care workers }(n=5)\end{array}$ \\
\hline & & 50 & $\begin{array}{l}\text { - An e-mail to all health care workers to promote the website will have no effect due to } \\
\text { e-mail overload and spam }(n=4)\end{array}$ \\
\hline & & 51 & - Consulting a co-worker is more efficient than looking up guidelines on a website $(n=11)$ \\
\hline & $\begin{array}{l}\text { Subjective } \\
\text { norm }\end{array}$ & 52 & $\begin{array}{l}\text { + Coworkers' opinion affects an individual health care workers' intentions to adopt the } \\
\text { website }(n=8)\end{array}$ \\
\hline & & 53 & $\begin{array}{l}\text { - Coworkers' opinions will not affect an individual health care workers' intention to adopt the } \\
\text { website }(n=12)\end{array}$ \\
\hline \multirow{3}{*}{$\begin{array}{l}\text { Behavioral } \\
\text { intention } \\
\text { (20 statements) }\end{array}$} & & 54 & + I will (continue) to use the website $(n=12)$ \\
\hline & & 55 & - I will not (continue to) use the website $(n=4)$ \\
\hline & & 56 & - I do not know yet whether I will (continue to) use the website $(n=4)$ \\
\hline
\end{tabular}

- Mentioned by at least three different respondents. + stands for facilitator, - for barrier. 
"Especially the search engine [...] when it functions well, and one hits the mark by entering the right keywords, then it is really easy."

(German physician; see Table 6.4., Category 12)

\section{Website's accuracy and disclosures}

On the contrary, respondents were more negative about the website's accuracy and disclosures, which seemed to negatively affect the website's credibility $(n=9)$. It was not clear to respondents $(n=3)$ how often information on the website was updated, and they also missed sound evidence that the website's guidelines were in accordance with the best available evidence $(n=3)$. Furthermore, credibility was seen as a problem because it was not obvious who created the website $(n=3)$. One respondent suggested to acquire accreditation to enhance the website's credibility.

\subsubsection{Statements related to predisposing (individual) factors}

\section{Website's target group}

Table 6.4. shows that 85 statements concerned individual factors, of which 58 were positive and 27 were negative. The majority of positive statements concerned respondents' ideas about the target group to whom the website would be most useful $(n=38)$. Respondents agreed that patients $(n=14)$ and nurses $(n=12)$ would benefit most from the website. Nursing staff perceived the website as useful because "this website is the first that handles the topic in such a profound manner," which enhances the website's credibility $(n=7)$, and "the practical information on the website complements the guidelines enforced at their hospital" $(n=4)$.

\section{Attitude toward the website}

43 statements were related to respondents' attitudes toward using the website. The majority of these statements $(n=27)$ were negative, and prevailed particularly among medical professionals (microbiologists and physicians). Negative attitudes involved resistance to web-based technologies $(n=5)$ and doubts about a possible discrepancy between guidelines communicated on the website and guidelines locally enforced $(n=10)$ :

“Each hospital has its own specific guidelines for particular situations. For instance, we don't have short-sleeved gowns for physicians, in contrast to what the website recommends."

(German medical microbiologist, see Table 6.4., Category 23)

Another subject of concern, in the respondents' opinion, was that the website can never replace face-to-face communication $(n=5)$ : 
"I assume it is better that when the nurse has time, and the physician has time, to answer such questions in a personal conversation. You cannot leave the worker with 'Google it yourself!' "

(German medical microbiologist; see Table 6.4., Category 24)

\section{Personality}

Another individual factor emerging from the interviews was personality. Four respondents expressed that "self-willed people are more inclined to try new things, such as a website" (see Table 6.4., Category 25).

\subsubsection{Statements related to enabling (work-related) factors}

\section{Tacit knowledge}

Compared to the other groups of factors, respondents expressed relatively few statements concerning work-related factors. The majority of the 53 statements were negative $(n=29)$. Ten respondents perceived no need for the website, as they had already become skilled in performing the guidelines $(n=10)$.

\section{Infrastructure}

Other negative statements involved a lack of computers with an Internet connection on the ward $(n=7)$ and high work pressure $(n=9)$, both of which would hinder consulting the website during working hours.

\section{Work experience and workload}

Conversely, almost all respondents $(n=17)$ indicated that "the website can easily coexist in daily work practices, as HCWs have become experienced with technology" (Table 6.4., Category 35). Even more contradictory to the finding that high work pressure impedes website use was that three respondents claimed the website could actually decrease infection control staff's workload:

"It saves us a lot of phone calls, actually. Actually the website takes away some of our work."

(Dutch infection control nurse; see Table 6.4., Category 30)

\subsubsection{Statements related to reinforcing (organizational) factors}

Of all 361 statements that can be found in Table 6.4., one third involved reinforcing factors. In contrast to the work-related and individual factors, about which respondents were mainly negative, respondents assigned a more positive role to the organization: go of 131 statements were positive. 


\section{Training and feedback}

Respondents were particularly favorable about the opportunity to promote the website during obligatory training sessions $(n=13)$ :

"We are able to rush through a lot of topics, for instance MRSA. We discuss the hospital's MRSA-rate, the main points, the ward, and the measures. And then we also discuss the website, we project it on the wall with a beamer and everyone can see it."

(German infection control nurse; see Table 6.4., Category 41)

\section{Management values}

Training courses are usually conducted by an infection control nurse. Half of the respondents indicated the infection control nurse to be the most influential person stimulating website use $(n=10)$. However, this positive influence of the infection control nurse was played down by eight respondents (all physicians or medical microbiologists) who expressed that infection control nurses have no influence on their behavior: "what she thinks does not matter", or "I don't care what she believes" (see Table 6.4., Category 39 and 40).

\section{Communication channels and subjective norm}

Furthermore, respondents were positive about the role of personal communication. Twelve respondents, of whom eight were nursing staff, perceived personal recommendations of the website among HCWs as an effective way to stimulate website use, as their colleagues' opinions influence their behavior $(n=8)$. Also, a personal approach from the website's project team was positively valued $(n=6)$ :

"Last week there was a meeting (...) and I attended it briefly. The nice thing about that was that I spoke to X [a member of the website's project team] (...) and he showed me the website. So now I have incorporated the website in my Favorites."

(Dutch medical microbiologist; see Table 6.4., Category 47)

On the contrary, eleven respondents perceived personal contact as more efficient than using the website.

\subsubsection{Statements related to behavioral intention}

Most respondents expressed willingness to use the website in the near future $(n=12)$. Four indicated no intention to use the website because they "already knew the answers" ( $n=2)$ or they supposed "information on a website does not fit the situationdependent character of an MRSA policy" $(n=2)$. The four remaining respondents expressed uncertainty about their intentions to use the website. 


\subsubsection{Summary of main findings}

In order to structure the results more conveniently, we summarized the main findings in Table 6.5.

\subsection{Discussion}

\subsubsection{Theoretical implications}

\section{Personal intention to use the website}

The results of this case study show that the usability, design, and relevance of the website with infection control guidelines were positively evaluated by its users. However, respondents were rather negative about the website's credibility. In particular, infection control professionals indicated that they would use the website only if there was enough guarantee that the guidelines on the website were in concordance with the best available evidence (accuracy), and if elements like terms of use, links to other sources, and liability (disclosures) were clearly presented. This supports the finding from previous research that communicating clinical practice guidelines online requires a special focus on credibility (Jeannot et al., 2003; Rousseau et al., 2003). Omitting credibility-enhancing features on a website might even lead to individual resistance among HCWs toward using the website, as 17 out of 43 interview statements about attitudes were related to credibility (Table 6.4., Category 18 and 23).

Next to credibility concerns, the personal intention to use the website was strongly affected by the experience and tacit knowledge of HCWs, which consists of their practical and experiential wisdom. Ten HCWs, including all medical or infection control professionals that participated in the study, indicated that they already had become sufficiently skilled in performing the guidelines, and therefore did not need the website. Therefore, these occupational groups deemed the website to be useful only for nursing staff and patients, but not for themselves. This finding is not surprising, as technology adoption in health care has always been associated with resistance among HCWs with more strategic tasks (McDonald et al., 2005; Parker \& Lawton, 2002). Previous research demonstrated HCWs to overestimate their knowledge of preventive measures and their own skills in applying them in everyday practice (Van Gemert-Pijnen et al., 2005). Furthermore, the website might be perceived as a threat to medical and infection control professionals respected authority: they might fear that HCWs will rather consult the website than turn to the professionals for help.

Nursing staff might be more willing to conform, as their views of professionalism are bound up with adherence to rules and guidelines, and they are therefore more inclined to adopt web-based guidelines than are physicians and infection control staff. Physicians and infection control professionals, on the other hand, are trained to use clinical judgment, and are encouraged to make decisions on a case-by-case basis. They therefore may perceive rules communicated on a website as a threat to professional autonomy. However, in order eliminate resistance and create ownership 
Table 6.5. Overview of main study findings, resulting from interview statements $(N=20)$

\begin{tabular}{|c|c|}
\hline Category & Main findings \\
\hline \multirow{2}{*}{$\begin{array}{l}\text { Website quality } \\
\text { (72 statements) }\end{array}$} & $\begin{array}{l}\text { - The usability and design were positively valued, next to the contextual relevance of the website's } \\
\text { information. }\end{array}$ \\
\hline & $\begin{array}{l}\text { The credibility of the website was negatively valued because it is not clearly stated whether the } \\
\text { website's guidelines are in concordance with the best available evidence. Next, the source is not } \\
\text { obviously disclosed. }\end{array}$ \\
\hline $\begin{array}{l}\text { Predisposing (individual) } \\
\text { (85 statements) }\end{array}$ & $\begin{array}{l}\text { Health care workers, particularly infection control professionals, might encounter resistance } \\
\text { toward the website because they prefer to use their personal knowledge and experience and } \\
\text { communication with colleagues over online sources. }\end{array}$ \\
\hline Enabling (work-related) & - High work pressure possibly impedes website use during work hours. \\
\hline (53 statements) & - On the other hand, website use might decrease work pressure for infection control professionals. \\
\hline $\begin{array}{l}\text { Reinforcing (organizational) } \\
\text { (131 statements) }\end{array}$ & $\begin{array}{l}\text { Interpersonal (face-to-face) communication between health care workers from different } \\
\text { occupational groups in both a formal (training) and informal (feedback) manner is the most } \\
\text { stimulating factor for website use. }\end{array}$ \\
\hline & - Infection control nurses are the most important opinion leaders to advocate website use. \\
\hline $\begin{array}{l}\text { Behavioral intention } \\
\text { (20 statements) }\end{array}$ & $\begin{array}{l}\text { Health care workers are willing to use the website in the future, unless they already are familiar with } \\
\text { the content or feel resistance against funding practical decisions on information from a website. }\end{array}$ \\
\hline
\end{tabular}


among infection control professionals toward the website, it is essential to more actively involve infection control professionals in the early phases of the design process (Kinzie et al., 2002; Van Gemert-Pijnen et al., 2005).

\section{Workload and intention}

The relationship between workload and intention to use the website appeared to be rather ambivalent. Almost half of the sample $(n=9)$ perceived high work pressure during working hours as an impediment to consulting the website. In contrast, three respondents suggested that the website might lower work pressure for infection control professionals, as they will receive fewer questions from HCWs. Additionally, website use seemed to be easily compatible with daily work practice, as 17 respondents mentioned that HCWs have become experienced with technology. This mixed effect of technology on HCWs has been identified in previous research. A study entitled "What Do Nurses Have to Say About Information Technology in Their Workplace?" demonstrated that nurses perceive an added value to technology, but at the same time "often feel exhausted and stressed out after dealing with technology in the workplace" (Ho \& Hovenga, 1999). Technology can create more work for HCWs, as it requires more knowledge and a precise understanding of the technology, which implies that HCWs must learn to use the technology.

\section{Interpersonal communication}

Another factor that can influence website adoption, both in a positive and negative sense, is interpersonal communication. The majority $(n=11)$ of respondents agreed that consulting a colleague would be more efficient than searching for guidelines on the Internet. Conversely, a personal recommendation of the website among HCWs was considered the second most effective facilitator of website adoption $(n=12)$. Interpersonal communication has been mentioned previously as the most effective communication channel for realizing change or stimulating adoption of new technology (Bero et al., 1998). Interpersonal communication provides an opportunity to tailor information to recipients (Williams \& Dickinson, 2008). Other suggestions were that the website could best be promoted during obligatory training sessions $(n=13)$ held by the infection control nurse, who was considered the most important opinion leader by half of the sample. These facilitators are all related to the group of organizational factors in the conceptual framework. Obviously, the organization contains features or elements that enable HCWs to more readily adopt a new technology. As most of the interview statements (131 out of 361 ) were related to this organizational category, and the majority of these statements were positive $(n=90)$, the organization appeared to be a paramount source of support and reinforcement for sustaining technology adoption by individual HCWs. This finding is in line with the assumptions of the PRECEDE model that we used as a framework for guiding our study, and which has also been demonstrated in previous studies (Dejoy et al., 2000; Kaplan, 1997; Kukafka et al., 2003). However, qualitative research does not enable us to draw conclusions about causal relationships or the strength of relationships between groups of factors in the framework, or about intentions to use the website. In this regard, quantitative results combined with structural equation modeling could 
be of major value.

\section{Value of the PRECEDE model}

The PRECEDE model appeared appropriate to study factors affecting HCWs' adoption of a website with infection control guidelines. The PRECEDE model, which normally is used in health education, enabled us to study website adoption from a multifaceted view. In contrast to other models such as UTAUT and Social-Cognitive Theory, PRECEDE acknowledges that a successful adoption-enhancing intervention should consider factors from multiple groups that are intertwined, starting with the organization (Kukafka et al., 2003). Our findings, seen from a PRECEDE-point-of-view, stratify the areas that will speed up the rate of adoption according to Rogers (2003):

- Reinforcing factors: When HCWs witness that opinion leaders and peers have adopted the website (subjective norm), it improves the prospect that the individual HCW will copy the behavior (observability). Next, when infection control nurses incorporate website use in their training sessions and let HCWs work with the website during training (training/feedback), HCWs can try out the innovation without total commitment and with minimal investment, which improves adoption rates (trialability).

- Enabling factors: If HCWs have become skilled in using technology during work practice (work experience), and sufficient computers are available on the ward (infrastructure), the ability of an innovation to coexist with technologies and social patterns already in place will increase, which improves the prospects for adoption (compatibility).

- Predisposing factors: When the individual HCW perceives no difficulties in using the website in practice (complexity), and anticipates to benefit from using the website (relative advantage), he/she is more likely to adopt the website.

\section{User-centered design}

Overall, our findings highlight the need for general efforts to systematically include end users of technologies in the design and production process (Kinzie et al., 2002). We could not have foreseen the factors respondents came up with, nor the fact that factors would differ so much between various target groups. Whereas infection control staff (in particular) suggested more abstract and strategic factors, nursing staff raised more practical ideas to stimulate adoption. Taking into account these target groups' perceptions can contribute to the eventual success of the technology (Kinzie et al., 2002). Engagement of those responsible for adoption is considered indispensable by Greenhalgh et al. (2004). These authors claim that people are not passive recipients of innovations. Rather, they seek innovations, experiment with them, evaluate them, find meaning in them, develop feelings about them, challenge them, worry about them, complain about them, "work around" them, gain experience with them, modify them to fit particular tasks, and try to improve or redesign them, often through dialogue with other users. 


\subsubsection{Practical implications}

\section{Multi-faceted intervention}

The aforementioned facilitators of technology adoption should be combined in an intervention. Multi-faceted intervention strategies have more chance of success than do single-faceted approaches or promotion programs focusing on one or two elements only. When various intervention strategies are used in combination, starting with organizational factors, they are probably more effective than interventions affecting individuals only (Pittet, 2000). Our study provides specific directions for such a multi-faceted intervention (see Table 6.6.).

\section{Enhance website's credibility}

First, it is important for the website design team to ensure that the website meets all HCWs' needs, of which credibility is the most salient. Table 6.6. summarizes the suggestions put forward by the HCWs in our sample. Another way to improve credibility is by applying for certification. The Health on the Net Foundation (2009) and the Dutch ZegelGezond (2009), for instance, have developed an accreditation system to improve the quality of medical and health information online. Several other initiatives have been undertaken to develop such quality markers, although their success has not yet been clearly determined (Rippen \& Risk, 2000). The website with infection control guidelines that we developed will participate in the pilot project of ZegelGezond, which will start in October 2009.

\section{Opinion leaders and workload reduction}

Second, medical and infection control professionals with resistance to new technologies should be predisposed favorably by emphasizing their roles as opinion leaders. For this purpose, they could be personally approached by the website design team. In order to trigger infection control professionals to advocate website use among HCWs, the website design team should underline in a personal conversation with target group members that website use can reduce work pressure for infection control staff (as HCWs will use the website to look up guidelines instead of seeking personal advice from the infection control nurse time after time). For this type of conversation between the design team and the infection control staff, persuasive interview techniques can be used. Persuasive interviewing incorporates communication dynamics to influence the behavior of the respondent (Rabon \& Chapman, 1994).

\section{Training sessions}

Third, when infection control staff are willing to promote website use, this should optimally be performed during obligatory training sessions for HCWs. Allowing HCWs to work with the website for several minutes during this training session will trigger them to use it in their daily work practice. The infection control nurse should also dispel HCWs' fear that work pressure is too high to consult the website during work hours. They can mention that, at first, it will take some time to familiarize oneself 
Table 6.6. Intervention directions to enhance the adoption of a website with infection control guidelines, as suggested by interview respondents $(\mathrm{N}=20)$

\begin{tabular}{|c|c|}
\hline Intervention directions & Responsible \\
\hline \multicolumn{2}{|l|}{ Website itself } \\
\hline $\begin{array}{l}\text { - Inform about the frequency with which guidelines are updated } \\
\text { - Emphasize that the guidelines provided by the website are in concordance with the best available } \\
\text { evidence by providing hyperlinks to original sources }\end{array}$ & $\begin{array}{l}\text { Website design team in col- } \\
\text { laboration with infection control } \\
\text { professionals }\end{array}$ \\
\hline $\begin{array}{l}\text { Warrant the accuracy of the guidelines provides by the website by keeping the website as up-to-date } \\
\text { as possible }\end{array}$ & \\
\hline - Explicitly clarify who created the website & \\
\hline \multicolumn{2}{|l|}{ Implementation of the website } \\
\hline $\begin{array}{l}\text { Make infection control professionals aware of their role as opinion leaders, and the importance of } \\
\text { them advocating website use }\end{array}$ & Website design team \\
\hline $\begin{array}{l}\text { Emphasize that promoting website use among health care workers can decrease the workload of } \\
\text { infection control professionals (as they will receive fewer questions when health care workers retrieve } \\
\text { the answers from the website) }\end{array}$ & \\
\hline - Promote the website during obligatory training sessions for all occupational groups & Infection control professionals \\
\hline - Enable health care workers to work with the website during these obligatory training sessions & \\
\hline - Encourage personal recommendations of the website among co-workers & Health care workers \\
\hline $\begin{array}{l}\text { - Promote website use among health care workers by providing leaflets, sending e-mails, and } \\
\text { stimulating infection control nurse to include a link to the website in locally enforced guidelines }\end{array}$ & Website design team \\
\hline
\end{tabular}


with the website, but that website use will yield time in the future when they would no longer have to consult a colleague or the infection control staff, and therefore it is worth the investment in learning to work with the website.

\section{Interpersonal communication}

Fourth, as interpersonal communication appeared to be the dominant way of stimulating website use among HCWs, it is important to ensure that HCWs mutually recommend the website. The website team can encourage this by reminding HCWs of the website's existence by supplying leaflets, e-mails, etc. However, in order to achieve successful implementation (in the sense that HCWs incorporate the website into their daily work routine), more is needed: interpersonal processes are most successful if they are collaborative, and therefore it is of vital importance to stimulate awareness that infection control is a collective management problem requiring the active involvement of infection control, medical, and nursing staff. By focusing on interpersonal exchange, network approaches can potentially facilitate the "predisposing, enabling, and reinforcing factors" required for the consistent and longterm adoption of best practices (Grol \& Wensing, 2004).

The most effective way to change infection control professionals' perception is by promoting personal interaction between nursing and infection control staff. Direct interaction permits the sharing of tacit knowledge, which is the practical and experiential wisdom HCWs have without being aware of it and that appeared to be a major barrier among HCWs to use the website. Ongoing interaction between peers might create a form of sense-making within the organization about the importance of guideline adherence and the website's benefits (Bosua \& Scheepers, 2007). When HCWs discuss the risks, health benefits, and dangers of nosocomial infections, all individuals (including physicians and infection control professionals) become more aware of the fact that they will be held responsible for their clinical practice (Fervers et al., 2005). In this way, direct interaction facilitates individual feelings of accountability, and consequently, rules and guidelines become effective mechanisms for social control (which will also facilitate website adoption). Such an approach is also called "knowledge sharing", which can be seen as a dual process of enquiring and contributing to knowledge through activities such as learning-by-observation, listening and asking, sharing ideas, giving advice, recognizing cues, and adopting patterns of behavior (Bosua \& Scheepers, 2007). An intervention based on this approach significantly reduced the MRSA rate in England. This approach differs from other types of interventions because of its involvement of all staff in its development and application. In addition, information exchange, employee participation, and social networking are emphasized during the intervention process (Cooper et al., 2005).

\subsubsection{Limitations}

The findings of this study are limited by the fact that the respondents were all familiar with the website and therefore were possibly more favorably predisposed toward website use compared to those who not know the website. Future research should involve both users and non-users. A second limitation of this study is the small sample 
size, which limits the ability to generalize the findings.

Although qualitative methods enabled us to optimally explore HCWs' perspective toward factors affecting website adoption, we could have benefited from quantitative techniques. Validated instruments such as UTAUT are available to measure determinants of technology adoption (Peters, 2007). However, it appeared that the scales for the UTAUT constructs are not robust and stable across settings and therefore are likely to effect the interpretation of results (Li \& Kishore, 2006).

Furthermore, we did not measure the effectiveness of the interventions strategies proposed in Table 6.6. Overall, the evidence on impacts of adoption-enhancing interventions is weak. Testing of intervention directions generated by this study may help establish evidence for such impacts (Grol \& Wensing, 2004). This remains a subject for future research.

\subsection{Conclusion}

Factors from multiple groups of factors determine HCWs' adoption of a website with infection control guidelines, but training and feedback appear to be a paramount reinforcer of initiation and maintenance of technology adoption. Since HCWs may prefer personal experience and communication with colleagues over online sources, it is of vital importance to construct a website that addresses HCWs' particular needs and ensures that the technology fits within the informal mechanisms of communication operative in HCWs' social networks. This can be realized through the involvement of potential adopters in the development and implementation process. 
A usable and well-implemented website with infection control guidelines in itself is not enough to enhance health care workers (HCWs)' adherence to guidelines. In order to change HCWs' adherence behavior, an intervention strategy that takes into account individual, work-related, and organizational factors is required. Therefore, a study was conducted to identify factors affecting the adherence to infection control guidelines, based on HCWs' perceived barriers and facilitators. Again, as in the previous chapter, the PRECEDE model (Predisposing, Reinforcing, and Enabling Causes in Educational Diagnosis and Evaluation) served as the theoretical framework. First, a questionnaire was conducted among $217 \mathrm{HCWs}$ to identify which barriers and facilitators determine their intentions to adhere to infection control guidelines. Second, 24 interviews with HCWs were administered to discover how these barriers can be used to stimulate adherence.

The questionnaire showed that safety climate (ward's management values and training/feedback) is a paramount reinforcer of guideline adherence. Individual (predisposing) and work-related (enabling) facilitators provide the direct motivation for adherence, but a supportive safety climate is necessary to realize this motivation. Additionally, the interviews identified training/feedback as the most important facilitator of safety climate and management values as the most important barrier. Tailored training/feedback should address the most salient predisposing barriers of HCWs' intention to adhere; that is, physician's negative attitudes toward the guidelines' usefulness and their low degree of conformity.

Since the German culture is characterized by a high degree of uncertainty avoidance, different scores were expected between German and Dutch HCWs with regard to the intention to adhere to guidelines. However, these differences were not detected, possibly because all respondents were employed in a hospital in the Dutch-German border region. It might be that HCWs working in border regions hold values that diverge from what is typical for their country and rather embrace a cross-border mentality. However, "cultural differences" were found on another level: between occupational groups. Nurses held more positive opinions toward guideline adherence than physicians. Based on the results, it is suggested that adoption-enhancing interventions should not be tailored to the norms and values inherent to HCWs' national culture but rather to the occupational group to which HCWs belong. 


\section{Chapter 7 \\ Identifying intervention strategies for guideline adherence based on health care workers' perceived barriers and facilitators}

Based on: Verhoeven F, Steehouder MF, Hendrix MGR, Peters O, Gemert-Pijnen JEWC van. Identifying intervention strategies for guideline adherence based on health care workers' perceived barriers and facilitators. Submitted to Journal of Hospital Infection. 


\section{Chapter 2}

Assess and analyze needs

\section{Chapters $3 \& 4$}

Rank and select needs

\section{Chapters 3 \& 4}

Identify solutions, Articulate goals

\section{Chapters $3 \& 4$}

Design and development

\section{Chapter 5}

Implement solution

\section{Chapter 6}

Users adopt solution

\section{Chapter 7}

Users realize goals 


\section{Identifying intervention strategies for guideline adherence based on health care workers' perceived barriers and facilitators}

\subsection{Introduction}

Although most health care workers (HCWs) are aware of the rationale for infection control guidelines, adherence is generally poor (Berhe et al., 2005). Several intervention models identify three groups of barriers and facilitators of guideline adherence: individual factors, like reluctance to change; work related factors, e.g., sufficient staff and time; and organizational factors, such as support from superiors. Adherence-enhancing interventions are most likely to be successful when facilitators from each group of factors are taken into consideration (Francke et al., 2008). Most models assign equal importance to the influence of each of these three groups of factors on adherence. However, considering that successful behavior change implies changes in the organization, work environment, and the people involved, it is important to examine such phenomena from a social interactionist perspective, which implies that the three aforementioned groups of factors mutually reinforce one another (Kaplan, 1997). For instance, efforts to motivate individual HCWs to adhere to guidelines may fail if management is not supportive. While other models assume the three separate group of factors only have direct effects on intended behavior, the PRECEDE model (Predisposing, Reinforcing, and Enabling Causes in Educational Diagnosis and Evaluation) suggests that there is also an indirect effect of organizational barriers and facilitators on intention to adhere. Moreover, these effects are mediated by work-related and individual facilitators and barriers (Dejoy et al., 2000). Therefore, we deem the PRECEDE model as appropriate to identify barriers and facilitators of HCWs' adherence to infection control guidelines.

In health care, the culture is still to implement single-faceted, expert-driven interventions, rather than multi-faceted interventions that fit HCWs' needs, often resulting in dissatisfaction and abandonment (Johnson et al., 2005; McCoy et al., 2001; Murphy 2002). Several approaches such as Intervention Mapping or the Breakthrough Series Collaborative enrol participants to articulate interventions goals (Grol \& Wensing, 2004). Although these approaches are effective in the short term, long-term improvements remain unclear (Glasgow et al., 2002). Furthermore, the approaches are particularly aimed at changing individual attitudes rather than creating organizational reinforcement, as the PRECEDE model suggests. In the current study, quantitative data will be used to answer which barriers and facilitators determine HCWs' intentions 
to guideline adherence, whereas qualitative data will tell how these barriers and facilitators influence intentions to adhere in daily practice and which intervention strategies can be used to enhance adherence.

\subsection{Methods}

\subsubsection{Research setting and study population}

The Dutch-German research project EUREGIO MRSA-net Twente/Münsterland addresses the rapid spread of Methicillin Resistant Staphylococcus aureus (MRSA) at a cross-national level, and the study reported here was conducted as part of this project (Friedrich et al., 2008). Therefore, MRSA guidelines served as a case study.

This study was conducted in two Dutch and two German hospitals involved in the research project, varying in size from 314 to 1500 beds. The researcher (FV) distributed confidential, self-administered questionnaires with return envelopes to a random sample of $320 \mathrm{HCWs}$. In the same time period, semi-structured interviews were conducted with a convenience sample of questionnaire respondents $(n=24)$.

\subsubsection{Data collection}

\section{Quantitative}

A self-administered questionnaire was developed, guided by the categories of the PRECEDE model (see Table 7.1.). The items were derived from existing valid instruments fitting the study themes and can be found in Appendix 1 (Dejoy et al., 2000; Gershon et al., 2000; McGovern et al., 2000). Item content was adapted to the national MRSA guidelines of the Dutch Working Party on Infection Prevention and the German Robert Koch Institute (Dutch Working party on Infection Prevention, 2005; The Committee for Hospital Hygiene and Infection Prevention at the Robert Koch Institute, 2002). These guidelines are recognized as standards of care in the concerned countries. The questionnaire was originally set up in Dutch and then translated into German. A seven-point Likert scale, ranging from "strongly disagree" to "strongly agree," was used for all item responses.

\section{Qualitative}

Subsequently, scenario-based interviews were administered with 24 questionnaire respondents ( 16 nurses, 8 physicians). We used 27 "what if" scenarios simulating the actual guideline performance, like: "You are attending to an MRSA patient in an isolation room. Which preventive measures must you take in order to not transmit MRSA to other patients or personnel?" Two medical microbiologists validated the scenarios. The use of scenarios appeared to be a valid method for exploring actual practice (Kolbe et al., 1996). For each scenario, we asked respondents to mention the barriers and facilitators of adherence and subsequently asked how these factors can be targeted in an intervention. All respondents were interviewed in their work setting. Each interview lasted strictly one hour. The interviews were audiotaped with prior 
consent of the respondents, and transcribed verbatim.

\subsubsection{Data analysis}

\section{Quantitative}

The questionnaire's face and content validity was tested by piloting it face-to-face with 20 HCWs. Data were analyzed using SPSS v.16.o. Internal consistency reliability analyses were performed to evaluate internal consistency. Structural equation modeling techniques were applied using the software AMOS 5 to examine the contribution of the three PRECEDE-categories to intention to adhere. Mann-Whitney $U$ tests were used to compute between-groups variation.

\section{Qualitative}

Two researchers (FV, JvG) independently divided each interview into separate statements and assigned codes to each statement based on the PRECEDE-categories. Inter-rater reliability on statement categorization was substantial (Cohen's kappa = 0.78) (Landis \& Koch, 1977). After coding all transcribed data, the number of times categories occurred in the data was counted.

\subsection{Results}

\subsubsection{Quantitative}

Initially, 252 questionnaires were returned (79\% response rate), and after a check for completeness, a final sample of 217 valid questionnaires was obtained. Of the respondents, $107(49.3 \%)$ were Dutch and 110 (50.7\%) were German. 153 respondents were female $(70.5 \%)$. Of the sample, $76.0 \%$ were nurses $(n=165)$ and $24.0 \%$ were physicians $(n=52)$. Mean age was 34.5 years $(S D=9.5)$. On average, respondents had been in their present job 9.0 years $(S D=8.3)$. Respondents worked in a surgery unit $(32 \% ; n=69)$, on internal medicine wards $(30 \% ; n=66)$, in an ICU $(30 \% ; n=65)$, or in paediatrics $(8 \% ; n=17)$.

In order to test the relation between the three PRECEDE-categories and intention to adhere, structural equation modeling techniques were used. Using a first-order confirmatory factor analysis, the measurement model estimated the extent to which the observed items loaded onto their respective latent variables. All latent constructs but no observed error variances were allowed to co-vary with one another. The measurement model of the PRECEDE model generated a good fit $\left(\chi^{2}(254)=399.57, \chi^{2}\right)$ $\mathrm{df}=1.57, \mathrm{SRMR}=.059, \mathrm{TLI}=.904, \mathrm{RMSEA}=.052$ [90\% confidence interval $[\mathrm{Cl}]: .042$, $.061])$. The internal consistency of the measures to explain intention to adherence was above aspiration level ( $\alpha>0.60)$ (Streiner \& Norman, 2003). The results obtained from testing the validity of a causal structure of the hypothesized PRECEDE model of intention to adhere to guidelines also showed an adequate fit $\left(\chi^{2}(266)=464.08, \chi^{2} / \mathrm{df}=\right.$ $1.75, \mathrm{SRMR}=.103, \mathrm{TLI}=.904, \mathrm{RMSEA}=.059$ [Cl: .050, .068]). The items in our structural model were discriminately valid, as the constructs were more closely related to 
their own indicators than to other constructs. Appendix 1 summarizes the original (uncorrected) mean and standard deviation, Cronbach's $\alpha$, the factor loading, and the squared multiple correlation of the observed indicators to adherence.

The model with standardized path coefficients showing the correlation between the different categories of the PRECEDE model and intention to adhere to guidelines is featured in Figure 7.1. The Figure shows significant direct effects of the enabling (work-related) and predisposing (individual) barriers/facilitators on intention to adhere. This implies that job satisfaction, attitude about the perceived benefit of adherence, and conformity facilitate a HCW's intention to adhere.

The same holds for "infrastructure," implying that the more readily available and accessible guidelines are to a HCW, the more likely he or she is to adhere. Furthermore, it appeared that when a HCW perceives that a high workload impedes the ability to adhere to guidelines, he or she is more inclined to adhere. The effects of reinforcing barriers/facilitators on intention to adhere are non-significant, indicating that safety climate aspects, like the ward's management concern for guidelines, do not affect adherence directly. However, Figure 7.1. shows an indirect effect mediated via the direct effect of reinforcing barriers/facilitators on enabling and predisposing barriers/facilitators $(\beta=.32)$. This means that individual and work-related facilitators provide the initial motivation for guideline adherence, but a supportive safety climate is necessary to realize this motivation.

Individual differences were primarily detected between occupational groups. Nurses were more inclined to adhere than doctors due to conformity values (Mann-Whitney $\mathrm{U}=2741, \mathrm{Z}=-3.9, p<.001$ ) and were more satisfied with their jobs (Mann-Whitney $\mathrm{U}=3484, \mathrm{Z}=-\mathbf{2 . 0}, \mathrm{p}<.05)$. Also, infrastructure was perceived better by nurses compared to physicians (Mann-Whitney $U=2772, Z=-3.9, p<.001$ ). Furthermore, significant differences between HCWs from different countries were found in the enabling and reinforcing category: workload was perceived more as a barrier to adherence by German HCWs compared to Dutch (Mann-Whitney U=4314, Z=-3.4, p<.01) and German HCWs perceived their safety climate significantly better than the Dutch (Mann-Whitney $\mathrm{U}=3018, \mathrm{Z}=-6.2, \mathrm{p}(.001)$.

\subsubsection{Qualitative}

We conducted semi-structured interviews with a convenience sample of 12 Dutch and 12 German HCWs. Eleven respondents were female and mean age was 39.3 years $(S D=10.0)$. Altogether, the respondents mentioned 135 facilitators and 276 barriers of adherence that could be related to the categories of the PRECEDE model (see Table 7.1.).

\section{Predisposing (individual)}

Out of 411 statements, $188(45.7 \%)$ involved the individual category. A negative attitude toward the guidelines' usefulness emerged as a salient barrier of adherence, considering the higher amount of negative statements compared to positive statements. Furthermore, respondents indicated that personality, like physicians' 
resistance to adhere, predominantly served as a barrier to adherence. Job satisfaction was not put forward at all by the respondents.

\section{Enabling}

Stress was only perceived as a barrier to guideline adherence, not as a facilitator. Infrastructure was not mentioned.

\section{Reinforcing}

Table 7.1. demonstrates that out of 411 statements, 123 (29.9\%) concerned safety climate, of which the vast majority involved statements about training/feedback. Training/feedback was brought up only in a positive sense. Wards' management values, on the contrary, were mainly perceived as a barrier. Management often sets forth an inconsistent policy that results in individual HCWs not knowing what to do and acting on their own accord. During the interviews, respondents proposed several intervention strategies to strengthen the safety climate, which can be found in Table 7.2 .

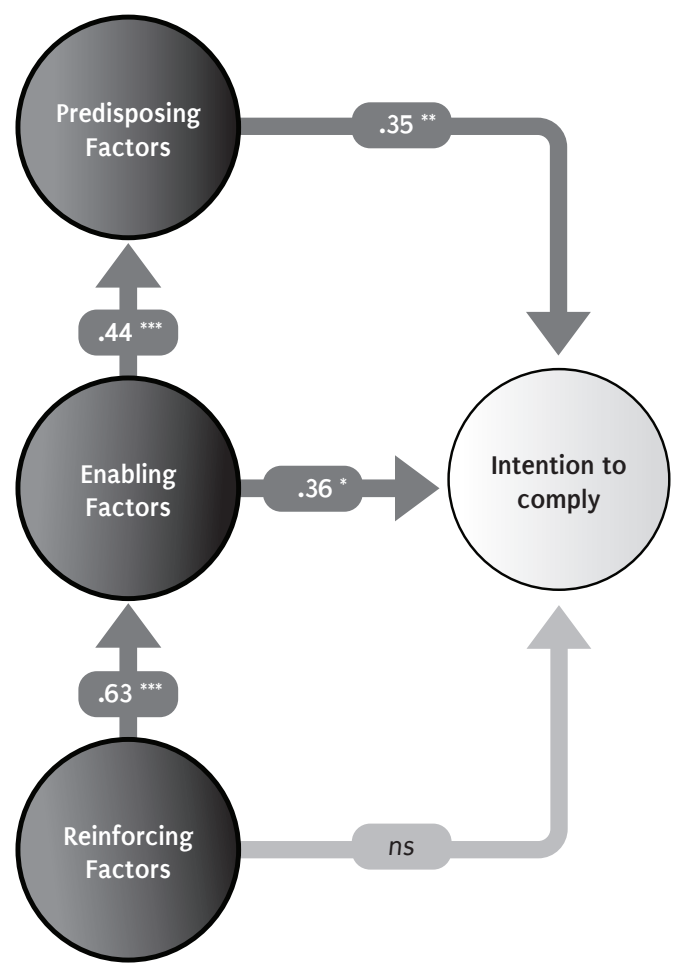

Figure 7.1. Standardized path coefficients showing the correlation between the different categories of the PRECEDE model and intention to adhere to infection control guidelines 
Table 7.1. Overview of barriers and facilitators of guideline adherence perceived by health care workers, expressed in number of interview statements $(\mathrm{N}=24)$

\begin{tabular}{|c|c|c|c|c|c|c|c|}
\hline \multirow[t]{2}{*}{ PRECEDE-category } & \multirow[t]{2}{*}{ Definition } & \multicolumn{2}{|c|}{ Barriers } & \multicolumn{2}{|c|}{ Facilitators } & \multicolumn{2}{|c|}{ Total * } \\
\hline & & $\#$ & $\%$ & $\#$ & $\%$ & \# & $\%$ \\
\hline \multicolumn{8}{|l|}{ Predisposing } \\
\hline Attitude & $\begin{array}{l}\text { The value or benefit anticipated from adherence to infec- } \\
\text { tion control guidelines relative to current practice, as } \\
\text { perceived by the individual HCW }\end{array}$ & 73 & 26.4 & 45 & 33.3 & 118 & 28.7 \\
\hline $\begin{array}{l}\text { Personality (show initiative/ } \\
\text { sense of responsibility) }\end{array}$ & $\begin{array}{l}\text { The degree to which an individual HCW is inclined to act } \\
\text { in accordance with some specified standard or authority }\end{array}$ & 68 & 24.6 & 2 & 1.5 & 70 & 17.0 \\
\hline Job satisfaction & The degree to which a HCW is happy with his/ her job & ० & ○ & o & o & o & o \\
\hline \multicolumn{8}{|l|}{ Enabling } \\
\hline Stress/workload & $\begin{array}{l}\text { The degree to which the amount of work needed to } \\
\text { be performed is feasible within a specific period, and } \\
\text { whether adherence to infection control guidelines is } \\
\text { compatible with this amount of work }\end{array}$ & 100 & 36.2 & 0 & 0 & 100 & $24 \cdot 3$ \\
\hline Infrastructure & $\begin{array}{l}\text { The extent to which infection control guideline docu- } \\
\text { ments (protocols) are readily available and accessible } \\
\text { to a HCW }\end{array}$ & 0 & o & o & o & 0 & 0 \\
\hline \multicolumn{8}{|l|}{ Reinforcing } \\
\hline Ward's management values & $\begin{array}{l}\text { The degree to which the opinion of an individual is } \\
\text { respected or listened to by others in terms of adherence } \\
\text { to infection control guidelines }\end{array}$ & 35 & 12.7 & 6 & 4.4 & 41 & 9.8 \\
\hline Training/feedback & $\begin{array}{l}\text { The degree to which individuals obtain the opportunity } \\
\text { to learn and discuss why and how to apply infection } \\
\text { control guidelines }\end{array}$ & o & o & 82 & 60.7 & 82 & 20.0 \\
\hline $\begin{array}{l}\text { Total number of interview } \\
\text { statements }\end{array}$ & & 276 & 100 & 135 & 100 & 411 & 100 \\
\hline
\end{tabular}

- Transcribed data of 24 interviews generated 411 interview statements related to barriers or facilitators of guideline adherence.

Table 7.2. Intervention strategies to enhance guideline adherence derived from interview statements ( $N=24)$

\begin{tabular}{ll}
\hline Target level & Intervention strategy (example from interview statements) \\
\hline $\begin{array}{l}\text { Reinforcing safety climate by adequate } \\
\text { training and feedback }\end{array}$ & $\begin{array}{l}\text { Once a year, a compulsory refresher course should be held during which the most important } \\
\text { parts of the guidelines are critically discussed, so health care workers' relapse into old (non- } \\
\text { adherent) routines is prevented. During this training class, the rationale behind adhering to } \\
\text { guidelines should be accentuated. }\end{array}$ \\
$\begin{array}{ll}\text { Training content: Emphasizing impor- } \\
\text { tance of adherence to infection control } \\
\text { guidelines despite high work pressure, } \\
\text { particularly among physicians }\end{array}$ & $\begin{array}{l}\text { adherent behaviors in situations of high work pressure, which, for instance, can be recorded by } \\
\text { hidden video cameras. }\end{array}$ \\
$\begin{array}{l}\text { Training content: Nurses should be } \\
\text { encouraged to point out physicians' non- } \\
\text { adherence }\end{array}$ & $\begin{array}{l}\text { Health care workers should be encouraged to practice guideline adherence during training class } \\
\text { (observed by the infection control nurse), so colleagues can give each other feedback about } \\
\text { performance. }\end{array}$ \\
$\begin{array}{l}\text { Training content: Creating risk perception } \\
\text { regarding risk of obtaining and transmit- } \\
\text { ting MRSA, particularly among physicians }\end{array}$ & $\begin{array}{l}\text { Worst-case scenarios should be mentioned during training sessions, e.g., what will happen in } \\
\text { a case of non-adherence? This will arouse health care workers' fear of consequences of non- } \\
\text { adherence. E.g., a health care worker that lost his/her job due to MRSA-carriership could share } \\
\text { his experiences during training class, so health care workers afterwards will be more inclined } \\
\text { to adhere to infection control guidelines since they want to maintain their job position. }\end{array}$ \\
\hline
\end{tabular}




\subsection{Discussion}

In this study, quantitative data showed that the ward's management values and training/feedback are paramount reinforcers of HCWs' intentions to guideline adherence. Management values and training/feedback are both features of safety climate, which is formed by the general perceptions of how safety is managed in the workplace (Guldenmund, 2000). Individual (predisposing) and work-related (enabling) facilitators provide the initial motivation for guideline adherence, but a supportive safety climate is necessary to realize this motivation.

Additionally, qualitative data told us how the barriers and facilitators affect HCWs' intentions to guideline adherence. Training/feedback appeared to be the most salient component of safety climate and facilitator of intention to adhere. Furthermore, physicians' negative attitudes and low degree of conformity were the most relevant predisposing barriers. Job satisfaction and infrastructure seemed to not play a role at all. Therefore, interventions aimed at enhancing guideline adherence should mainly focus on reinforcing safety climate by tailoring training/feedback to HCWs' needs so that negative attitudes and low conformity rates can be addressed. In this way, a single-component intervention (training sessions) can reach results at multiple levels. A possible training method is Organizational Behavior Management in which educational sessions are combined with reminders, regular refreshers, and continuous feedback (Cooper et al., 2005). The explicit, joint discussion of the risks of non-adherence between nurses and physicians might create individual feelings of accountability.

Based on our findings, we recommend tailoring adherence-enhancing intervention strategies to barriers and facilitators experienced by HCWs, because our study demonstrated that HCWs attach a different value to barriers and facilitators affecting guideline adherence than reported in existing literature. Tailoring interventions to target group's needs has been advocated before (Grol \& Wensing, 2004). In contrast to the usual "top-down" development process of expert-driven interventions, the intervention should be based on a "bottom-up" approach that allows HCWs to clarify their tacit knowledge and empowers them to indicate strategies for improvement.

We expected German and Dutch HCWs to score differently on personality in the questionnaire, based on Hofstede (1994). Since the German culture is characterized by a high degree of uncertainty avoidance and people are more inclined to rule adherence, we anticipated German HCWs to be more conforming than Dutch HCWs; however, significant differences were not seen. Our research was conducted in a border region where patients and HCWs cross the border daily to receive and provide health care. It might be that HCWs working in border regions hold values that diverge from what is typical for their country and rather embrace a cross-border mentality.

The interviews showed that the majority of barriers and facilitators of adherence identified in the literature play an actual role in practice. However, according to the literature, management values are the major facilitator of safety climate (McCoy et al., 2001), but our interview respondents suggested that training/feedback was more 
relevant and even labelled management values as a barrier. This contrasting finding calls for further investigation. Furthermore, the non-conforming personalities of physicians next to physicians' negative attitudes emerged to be relevant barriers of guideline adherence. The finding that nurses are more conforming than physicians may be due to nurses' views of professionalism as bound up with adherence to rules and guidelines. Physicians, on the other hand, are trained in the use of clinical judgment and encouraged to make decisions on a case-by-case basis, and therefore may perceive guidelines as a threat to professional autonomy (McDonald et al., 2005). While job satisfaction and infrastructure have previously been identified to affect intention to adhere significantly (Dejoy et al., 2000; Francke et al., 2008), our respondents did not raise these factors at all.

\subsubsection{Limitations}

Although our data adequately fit the PRECEDE assumptions (the model explained $45 \%$ of the variance), specific tests of PRECEDE have been limited, which is an important critical comment regarding the model (Mullen et al., 1987). There are very few guidelines regarding the measurement strategy and the number of variables is somewhat arbitrary and should be treated as a rough approximation. We consider PRECEDE however as the most appropriate conceptual framework because other health behavior models such as the Theory of Planned Behavior and the Health Belief Model focus only on predisposing facilitators and barriers and leave the reinforcing effect of the organization out of consideration. In other words, other health behavior models generate a train of single-faceted intervention strategies aimed at influencing one single factor (like attitude), whereas PRECEDE identifies a set of intervention strategies that mutually strengthen each other's effect.

Other limitations of our study were the relatively small sample size and limited geographic region, implying the impossibility of generalizing the results to all hospitals in Germany and the Netherlands. Also, the voluntary questionnaire and interview format could have been subject to several response biases. HCWs with strong feelings may have been more likely to complete the questionnaire and to take part in the interviews, and there is a concern regarding socially desirable responses, reflected by the positive average scores on all constructs (see Appendix 1).

\subsubsection{Future research}

In future research, it would be interesting to investigate the role of technology in the improvement of guideline adherence. Since an efficient format of instructional materials promotes learning (Clark et al., 2006), it may be interesting to develop an e-learning program aimed at increasing HCWs' knowledge regarding infection prevention and control. In industries such as aviation and nuclear power plants, e-learning courses have proven to be effective in motivating employees to work safely (Bates et al., 2003).

Also, an adherence-enhancing intervention can benefit from websites that can tailor evidence-based guidelines to HCWs' needs and assist with clinical decision-making. 
Verhoeven et al. (see Chapters 3 and 4 ) developed a website that provides HCWs with MRSA guidelines, communicated in an action oriented manner and presented multimodally. Embedding such a website in the hospital's training program might improve safety climate and guideline adherence. This is a subject for future research. 
Appendix 1. Questionnaire. Descriptive statistics, factor loadings, squared multiple correlations, and Cronbach's alpha of the observed indicators to explain intention to adhere to infection control guidelines ( $N=217)$

\begin{tabular}{|c|c|c|c|c|}
\hline Item & $\mathbf{M}$ & SD & $\beta$ & $\mathbf{R}^{2 \mathrm{a}}$ \\
\hline Intention to adhere $(\alpha=.65)$ & & & & .45 \\
\hline In the future, it is very likely that I will disinfect my hands before and after each patient contact. & 6.26 & 1.28 & .53 & .28 \\
\hline $\begin{array}{l}\text { In the future, it is very likely that I will disinfect medical equipment before and after I used them each } \\
\text { time. }\end{array}$ & $5 \cdot 75$ & 1.63 & .65 & .43 \\
\hline $\begin{array}{l}\text { In the future, it is very likely that I will point out a colleague that is not acting in accordance with the } \\
\text { safety precautions. }\end{array}$ & 6.02 & 1.31 & .81 & .65 \\
\hline Predisposing & & & & .20 \\
\hline Attitude $(\alpha=.73)$ & & & .59 & .35 \\
\hline A protocol is the best way to prevent the spread of MRSA in the hospital on a large scale. & 6.07 & 1.23 & .66 & .43 \\
\hline An MRSA-protocol should be obligatory for all employees. & 6.55 & 0.83 & .88 & .77 \\
\hline A protocol is of greater value if employees are involved in the development process. & 6.58 & 0.81 & .68 & .46 \\
\hline Personality $(\alpha=.82)$ & & & .79 & .62 \\
\hline $\begin{array}{l}\text { He believes that people should do what they are told. He thinks people should follow rules at all times, } \\
\text { even when no one is watching. }\end{array}$ & 3.98 & 1.32 & .87 & .76 \\
\hline $\begin{array}{l}\text { It is always important to him to behave properly. He wants to avoid doing anything people would say is } \\
\text { wrong. }\end{array}$ & 3.80 & 1.36 & .82 & .67 \\
\hline $\begin{array}{l}\text { It is important to him to be obedient. He believes he should always show respect to his parents and to } \\
\text { older people. }\end{array}$ & 3.58 & 1.20 & .76 & .58 \\
\hline It is important to him to be polite to other people all the time. he tries to never disturb or irritate others. & 4.03 & 1.17 & .70 & .48 \\
\hline Job satisfaction $(\alpha=.79)$ & & & .57 & .32 \\
\hline This hospital is a good place to work. & 5.61 & 1.37 & .87 & .76 \\
\hline I am proud to work at this hospital. & 5.15 & 1.54 & .84 & .70 \\
\hline I like my job. & 6.14 & 1.07 & .58 & .33 \\
\hline Enabling & & & & .40 \\
\hline Workload $(\alpha=.76)$ & & & .72 & .52 \\
\hline $\begin{array}{l}\text { When my workload becomes excessive, it is impossible for me to adhere to the measures in the MRSA } \\
\text { protocol. }\end{array}$ & $5 \cdot 55$ & 1.76 & .69 & .48 \\
\hline The occasions during which I worked unsafely were because I had to do the job quickly. & 5.67 & 1.79 & .77 & .60 \\
\hline If I always had to think about adhering to the protocol, I would not be able to perform all my duties. & 5.75 & 5.75 & 69 & .47 \\
\hline Infrastructure $(\alpha=.89)$ & & & .56 & .31 \\
\hline On this ward, the MRSA protocol is routinely available to me. & 5.53 & 1.62 & .87 & .75 \\
\hline When looking up information in the MRSA protocol, I am able to obtain this information quickly. & $5 \cdot 35$ & 1.67 & .95 & .91 \\
\hline I use the protocol in case I want to quickly look up something I'm uncertain about. & $5 \cdot 36$ & 1.71 & .75 & .57 \\
\hline \multicolumn{5}{|l|}{ Reinforcing } \\
\hline Safety climate (ward's management values and training/feedback) $(\alpha=.88)$ & & & &.$\circ 0$ \\
\hline The managers on my unit show great concern for the MRSA protocol. & $5 \cdot 71$ & 1.48 & .76 & .57 \\
\hline The managers on my unit think it is important to adhere to the measures in the MRSA protocol. & 6.34 & 1.10 & .79 & .62 \\
\hline Managers on my unit do their part to ensure employees adhere to the MRSA protocol. & 5.69 & 1.51 & .84 & .70 \\
\hline $\begin{array}{l}\text { Managers on my unit do their part to ensure employees will not become colonized or infected with } \\
\text { MRSA. }\end{array}$ & 5.96 & 1.39 & .81 & .66 \\
\hline On my unit, supervisors correct uninfection control guidelines. & 5.22 & 1.65 & .69 & .47 \\
\hline On my unit, employees are being checked based on protocol adherence. & 4.62 & 1.89 & .61 & .37 \\
\hline
\end{tabular}


${ }^{a}$ The $\mathrm{R}^{2}$ of a latent dependent predictor is the percent of the variance in the latent dependent variable accounted for by the latent independent variable. The $\mathrm{R}^{2}$ of an observed indicator is the estimated percent variance explained in that variable. In other words, the error variance of a variable is approximately 1 minus the percent of the variance of the variable itself. 
In this chapter, overall conclusions will be drawn from the findings reported in the previous chapters. Both theoretical and methodological implications are discussed, next to practical recommendations for both usability practitioners and infection control professionals. The chapter ends with directions for future research.

Overall, the reported studies demonstrated that a shift in the function of guidelines from regulation towards the communication of safe work practices led to more effective and efficient guideline retrieval among health care workers (HCWs). Involvement of intended users in the design process enabled us to tailor guideline communication to the target group's tacit knowledge. However, experts (infection control professionals) saw to the maintenance of the evidence-based, medical content. Next, we showed that it is crucial to consider organizational factors when implementing the user-driven guidelines and take into account value differences between occupational groups. How to actually perform the design and implementation process of user-driven communication of guidelines is explained in this thesis.

Future research should address the tailoring of web-based guidelines for the improvement of HCWs' knowledge base via more advanced technologies, such as an interactive dialogue system, e-learning modules, or decision-support tools. In this way, the right knowledge will be delivered at the right time in the right place, leading to improved quality of care. 


\section{Chapter 8 \\ Conclusions and discussion}





\section{Conclusions and discussion}

This chapter starts with overall conclusions. The second section discusses the theoretical implications based on the study's main findings. The third section deals with methodological implications. The practical recommendations for usability practitioners and infection control professionals are described in the fourth section, and this chapter ends with directions for future research.

\subsection{Conclusions}

\subsubsection{A website facilitates the successful retrieval of guidelines}

The research in this thesis demonstrated the feasibility of designing a website that enables HCWs to efficiently and effectively retrieve infection control guidelines and apply the guidelines to daily practice. The website outperformed traditional, paperbased guidelines with regard to time (efficiency), effort (efficiency), and the rate (effectiveness) with which HCWs retrieved infection control guidelines, the quality of the information (effectiveness), and user satisfaction.

The website allowed HCWs to search for an answer to contextually relevant questions in an elegant and intelligent way. The guidelines were presented multimodally, in an action oriented manner with links to additional resources, tables, figures, and videos (see Chapter 3). In this way, the guidelines were communicated to dovetail with HCWs' tacit knowledge and needs.

\subsubsection{User-driven design approach leads to an efficient and effective website}

The website's success might predominantly be ascribed to its user-driven design process. According to HCWs, the traditional paper-based documents appeared to be expert-driven (Chapters 2 to 4 ), and HCWs indicated their preference for a more practice-based, action oriented communication mode of infection control guidelines (Chapters 4 to 6). The "regulation function" of the traditional, expert-driven guidelines therefore had to be communicated differently in order to prevent HCWs not being able to apply the guidelines in practice. If guidelines are not communicated according to HCWs' perspective and do not fit into HCWs' perception of work practice, HCWs may become resistant towards using the guidelines, since they prefer to use tacit knowledge, experience, and communication with colleagues over online sources, as was shown in Chapter 6. Furthermore, it may be that HCWs' involvement in the design process creates ownership, fosters applicability of the website, and leads to a willingness to integrate the website into routine care (Van Gemert-Pijnen, 2003).

We primarily selected real users to participate in the user-driven design process. In 
practice, however, expert evaluations are generally more popular than user testing, mainly because they are less time consuming and less expensive (De Jong \& Lentz 2006). However, since expert evaluation does not show how users really behave, and since previous research has already demonstrated that the expert-driven format of infection control guidelines generated problems (Van Gemert-Pijnen, 2003), we decided to concentrate on user evaluation. We did however consult experts (infection control professionals) to comment on and revise the website's clinical content. It should be emphasized that the content of the guidelines was not subject to change during the design of the website. We solely adapted the presentation and structure of the guidelines according to HCW's tacit knowledge, but we consistently considered the evidence-based, medical content of the guidelines (Sackett et al., 1996). Without the assistance of infection control experts, the accuracy of the guidelines could not have been safeguarded.

\subsubsection{Organizational factors affect successful website adoption}

Despite the demonstrated value of the website (see Chapters 3 to 5 ), numerous obstacles to website adoption in daily work practice remain. The PRECEDE model was used to investigate factors affecting website adoption (Chapter 6) and guideline adherence (Chapter 7). The two studies showed that in both contexts, individual (predisposing) and work-related (enabling) facilitators provide the initial motivation for guideline adherence and website adoption, but a supportive safety climate is necessary to allow this motivation to be realized. Merging the findings from Chapters 6 and 7 allowed the development of an intervention strategy in which the website can be deployed to create a collective awareness toward the importance of guideline adherence. This intervention strategy will be presented in section 8.4.2.

Chapters 6 and 7 demonstrated that the factors affecting website adoption and guideline adherence as displayed in the literature are practically relevant. However, it turned out that HCWs attach a different value to barriers and facilitators than existing literature and experts do. For instance, according to the literature, management values are the major contributor of guideline adherence (Kermode et al., 2003; McCoy et al., 2001), but the findings in Chapter 7 suggest that HCWs perceive training and feedback as more relevant.

\subsubsection{Cultural differences do not exist on the national level, but on the occupational level}

Although some scholars advocate that communication means should be tailored to the norms and values inherent to the target group's national culture (Marcus \& Gould, 2000), we did not find major differences between HCWs from different countries in contrast to our expectations. Since the German culture is characterized by high degrees of uncertainty avoidance and power distance compared to the Netherlands (Hofstede, 1994), it was anticipated for German HCWs to hold more positive attitudes towards guideline adherence (see Chapter 1, section 1.2.1). In contrast to the national 
level however, the cultural differences appeared to be on the occupational level. We therefore advocate dovetailing communication of infection control guidelines with the needs of various occupational groups rather than with the needs of HCWs from different countries. The absence of cultural differences on the national level increases the chance of success for a global health perspective on the control of health careassociated infections (see Chapter 2).

\subsection{Theoretical implications}

\subsubsection{Shift in the function of guidelines as a communication means}

Infection control guidelines have two hardly reconcilable functions. They serve simultaneously as documentation of the health and safety policy of the healthcare institution ("regulation function") and as a means of communication for individual HCWs ("communication function"). Since the first function prevails, the second function often plays a subordinate part, leading to HCWs who have to act upon their own insights, since the guidelines do not provide an answer to context-specific questions.

Feelings of annoyance and frustration towards guidelines with a "regulation function" can be prevented by putting more emphasis to the "communication function", as was shown by this thesis's research. In other words, the format (content, structure, and presentation) of guidelines should range somewhere between a rule-based protocol, which provides clarity about "do's and don'ts," and a knowledge-based protocol that can be used as a decision-making tool (Berg, 1997). Putting forward the "communication function" does not imply that the "regulation function" disappears. Instead, safety regulations and legislation are communicated to support the guidelines, and do not longer function as the guidelines themselves.

Trying to change the focus of guidelines from the "regulation function" to the "communication function" is a challenge that also holds for other disciplines. In countless organizations, politicians, managers, safety officers, and work planners are involved in the control of safety by means of laws, rules, and instructions that are formalized means for the ultimate control of some hazardous process. They seek to motivate workers and operators, to educate them, to guide them, or to constrain their behavior by rules, so as to increase the safety of their performance. To be operational, the rules have to be interpreted and implemented in the context of the particular organizations, considering the work processes. However, the details drawn from local conditions and processes are often neglected in the communication of these rules, leading to unsafe situations (Rasmussen, 1997). Since situations are often dynamic, universal rules are inapplicable, and workers have to act upon their own insights. In order to prevent risky situations, it is of vital importance to tailor the communication of rules to the actual context in which they have to be adhered to. Guidelines that merely emphasize safety regulations are effective in industry but not 
in health care. Safety in health care differs in two respects from safety in industry: (1) the patient is at the center of discussion instead of a product, and (2) HCWs are not steps along an assembly line or cogs in a machine producing a product; they are professionals who apply knowledge and adapt learned procedures. Particularly in the health care setting, situations are characterized by a context-specific nature, implying that the communication of rules and guidelines should enable HCWs to use judgment at each step of the care process. In order to ensure that guideline communication represents the context in which the safe work practices should be administered, HCWs have to be involved in the design process.

\subsubsection{Involve HCWs in the design process}

A plausible explanation of the poor performance of expert-driven guidelines is that in the process of development and implementation of the protocols, the authors did not sufficiently take into account the differences in knowledge and needs of the various groups of HCWs (see Chapters 2 to 4 ). These findings are not restricted to this thesis's research: earlier research has already pointed out that protocols aimed at MRSA prevention and exposure to blood-borne pathogens were expert-driven (Van GemertPijnen et al., 2005; Van Gemert-Pijnen et al., 2006).

In health care, the culture is still to implement single-faceted, expert-driven interventions rather than multi-faceted interventions that fit HCWs' needs, often resulting in dissatisfaction and abandonment (Johnson et al., 2005; McCoy et al., 2001; Murphy 2002). Where previous studies only advocated the involvement of HCWs in the design process of guideline communication, they did not actually do so (Van GemertPijnen, 2003; Grol, 2001; Gross et al., 2001). As far as we know, our study was one of the first attempts to actually employ a user-driven design process to design guideline communication, drawing causal links between the design approach, the quality of the guideline communication, and their efficiency and effectiveness. We showed, as in line with previous research, that user-driven design is important to create ownership and to foster the applicability of guidelines. Therefore, the development of means to communicate infection control guidelines should not be the exclusive responsibility of infection control professionals (Gross et al., 2001; McCoy et al., 2001; McGovern et al., 2000; Murphy, 2002). However, as we have claimed repeatedly during this thesis, medical microbiologists (experts) should always verify the guidelines' clinical content.

\subsubsection{Consider the context of use}

Infection control guidelines themselves are hardly helpful in terms of stimulating guideline adherence (Elling, 1991; Van Gemert-Pijnen, 2003). More suitable means are organizational activities, such as management support, training, and communication. Organizational activities are crucial to ensure that HCWs become aware of the infection control guidelines and are ready and able to implement the required precautions (Dejoy, 2000). Generally, although a wide range of activities have been suggested to facilitate the implementation of infection control guidelines in practice, all that happens is that the protocol is brought to the attention of the users and a rudimentary instruction is given. Argumentation and feedback only occur incidentally, 
and there is usually no specific plan for introducing the guidelines, despite such a plan being a requirement of the guideline development policy (Gross et al., 2001). Furthermore, training is usually content-driven instead of action-driven (Van GemertPijnen, 2003).

The implementation of infection control guidelines should be a permanent feature in the hospital environment in order to ensure that HCWs behave in accordance with the required precautions and do not lapse into old routines. This thesis provides an intervention strategy to realize this (see Chapters 6 and 7 and section 8.2.).

We not only involved users in the design process but also asked them which factors affect a successful implementation process. This was found to be helpful, since users raised factors that differed from those stated by existing literature. Besides, by involving HCWs in the design and implementation process, safety awareness was created among HCWs, stimulating intensive discussion of safe behavior and thus guideline adherence. In this way, the research method in itself became an intervention strategy.

A theoretically guided approach can favorably influence implementation strategies. Therefore, we used the PRECEDE model to identify factors affecting successful implementation. Several other approaches to develop intervention strategies, such as Intervention Mapping or the Breakthrough Series Collaborative are available. Such approaches describe a user-involved methodology for the development of theory- and evidence-based health promotion programs, but they are not aimed particularly at the development and implementation of technology (Bartholomew et al., 1998; Cooper et al., 2005; Kok et al., 2004). Although these approaches have dramatic effects in the short term, it remains to be seen whether the changes reported will translate into long-term improvements (Glasgow et al., 2002). Furthermore, the approaches are particularly aimed at changing individual attitudes rather than creating organizational reinforcement.

\subsubsection{Consider cultural differences between occupational groups}

Nearly each sample of the studies incorporated in this thesis's research involved HCWs from two countries: Germany and the Netherlands. Given that the German culture is characterized as having a high degree of uncertainty avoidance compared to the Dutch culture (Hofstede, 1994), we anticipated detecting different needs toward guideline communication and a different degree of conformity with guidelines between Dutch and German HCWs (see Chapter 1, section 1.2.1). Cultures that score high in uncertainty avoidance prefer rules and structured circumstances, and these cultures try to minimize the possibility of unstructured, novel situations by strict laws and rules, safety, and security measures. It therefore was anticipated that German HCWs would revert themselves in safety rules and derive their behavior more from protocols and legislation to avoid uncertainty and justify their way of acting compared to Dutch HCWs. This expectation was strengthened by the findings reported in Chapter 2; the way in which national infection control guidelines were communicated differed 
substantially between Germany and the Netherlands. Whereas the German guidelines emphasized the legislative and evidence-based aspects ("regulation function"), the Dutch guidelines incorporated more information- and communication-oriented elements such as decision trees ("communication function").

However, in contrast to these expectations, no significant differences were detected on the national level, neither with regard to guideline adherence (Chapter 7). Also, we did not identify national cultural differences with regard to website evaluation (Chapters 3 to 6). Although previous research demonstrated that cultural background correlates with the preference for a particular website design (Marcus \& Gould, 2000), Dutch and German respondents participating in this thesis's research appeared to hold the same opinion regarding website design (Chapter 4 ), menu structure (Chapter 3), and website quality (Chapter 6). A possible explanation for the absence of cultural differences might be the cross-border region where (nearly) all studies were administered. In this region, many patients and HCWs cross the border daily to receive and provide health care (Friedrich et al., 2008). It might be that HCWs working in border regions hold values that diverge from what is typical for their country and rather embrace a cross-border mentality.

Although cultural differences on the national level remained absent, we detected "cultural differences" between occupational groups. Chapter 6 demonstrated that nurses were more willing to adopt the website in daily work practice compared to physicians and infection control professionals. Moreover, Chapter 7 reported on nurses' stronger intention to adhere to infection control guidelines compared to physicians. Nursing staff might be more willing to conform to both website use as well as guideline adherence, as their views of professionalism are bound up with adherence to rules and guidelines, and they are therefore more inclined to change current routines than are physicians and infection control professionals. Physicians and infection control professionals, on the other hand, are trained to use clinical judgment and are encouraged to make decisions on a case-by-case basis. They therefore may perceive rules communicated on a website as a threat to professional autonomy (McDonald et al., 2005; Parker \& Lawton, 2000). We therefore claim that interventions aimed at behavior change should be tailored not to the norms and values inherent to the target group's national culture but to the occupational group to which they belong.

\subsection{Methodological implications}

\subsubsection{Employ usability testing and inquiry rather than usability inspection}

A successful design process leading to a usable end product is characterized by its goal setting and the available time, budget, and expertise (Kinzie et al., 2002). Above all, it is crucial to select appropriate methods from the wide array of available usability research methods to investigate users' opinions (Nielsen, 1993). 
Usability research methods can be divided into three categories: inquiry, inspection, and formal usability testing. While the first and last involve real users, the second does not. Hom (1998) suggested that in usability testing, representative users work on typical tasks using the system (or the prototype), and the evaluators use the results to see how the user interface supports the users to do their tasks. In usability inquiry, usability evaluators obtain information about users' likes, dislikes, needs, and understanding of the system by talking to them, observing them using the system in real work (not for the purpose of usability testing), or letting them answer questions verbally or in written form. We tried to benefit from both these types of usability research: in the studies reported in Chapter 4, usability testing was used to investigate the website's usability before it was launched online. Respondents were asked to solve MRSA-related scenarios by means of using one specific website, i.e., they searched in a "closed" domain. In the study described in Chapter 5, we employed usability inquiry by letting respondents solve the same scenarios as described in Chapter 4, but this time, no restrictions were imposed to the website they had to use, i.e., they "searched freely". In other words, the purpose of research was not longer to test the website's usability but to inquire the functioning of the website in the "real world".

Ideally, usability testing and usability inquiry are applied concurrently. Since usability testing represents the "customizable user role" of the user-centered design approach and usability inquiry corresponds to the "environmental focus" of the contextual design approach, the combination adequately matches the bipartite approach of userdriven design that we advocated in Chapter 1, section 1.2.2.

The Card Sort method, prototyping interviews, and scenario-based tests, which are all methods of usability testing and usability inquiry, contributed to the insights in user's mental models. These insights could probably not have been generated by using usability inspection methods, which are more popular than usability testing and inquiry. In usability inspection, usability specialists and sometimes software developers or other professionals examine usability-related aspects of a product or system. Various methods of inquiry are available, such as heuristic evaluation or cognitive walkthroughs (Nielsen, 1994). Since it is often thought that experts are not able to identify real user problems (De Jong \& Lentz, 2006), and given our assumption that problems with the quality of guideline communication were particularly caused by their expert-driven character, we excluded experts from our design process.

This does not imply that we reject the use of usability inquiry or expert evaluation in the design process of communication means. We acknowledge these methods' primary benefits in that they are less expensive than other types of usability engineering methods and require fewer resources (Nielsen, 1994). Above all, experts have expertise and knowledge that users do not have, and particularly in the design phase of a website with clinical practice guidelines, expert knowledge is important. Therefore, we involved three usability experts in the mock-up interviews and had the website's content validated by medical microbiologists (see Chapter 3). For the purpose of this thesis's research however, we perceived to benefit more from user evaluation (usability inquiry and testing) rather than from expert evaluation (usability 
inspection).

\subsubsection{Employ scenario-based tests to collect user feedback}

In previous research, several strategies have been administered to collect user feedback on safety rules, like written commentaries on safety rules under revision and users suggesting improvements during regular working meetings (Elling, 1991). Although both strategies led to better safety rules according to users, the design process remained an expert-driven matter, since users did not get to see and evaluate the improved guideline format. Additionally, no feedback was obtained regarding the applicability of the guidelines in daily work practice.

It would therefore be advisable to conduct scenario-based tests rather than meetings, written comments, questionnaires or interviews to gather user feedback on communication means with safety rules. Scenario-based tests proved to generate helpful user feedback, leading to more efficient and effective communication of guidelines. Scenario-based tests provide a clearer and more authentic representation of daily work practice than any other method (Van Gemert-Pijnen, 2003).

\subsubsection{Involve not only end-users in the design process, but also other stakeholders}

Designers that decide to engage users in the design process, are usually inclined to only involve end-users in the process, neglecting other possible relevant individuals. Kinzie et al. (2002) specified the term end-user as "the individual who ultimately will use the system developed." Other relevant individuals are called stakeholders, but these stakeholders generally are not involved in the design process. Although endusers will primarily use the product or system, there will be also other individuals who will intermittently interact with the product or system: these are the stakeholders. It is crucial to involve these stakeholders in usability testing and inquiry. Therefore, we engaged not only HCWs in our research but also usability experts (Chapter 3), laymen (Chapter 3), and infection control professionals (Chapter 6).

Williams and Dickinson (2008) claim that involving users in the design and implementation process should not be a goal in itself. Researchers should critically reflect upon which end-users and stakeholders they want to involve in the single phases of the design process. Different end-users and stakeholders have various interests, and therefore, stakeholders and end-users should be selected for involvement in the design process according to their expertise.

\subsection{Practical implications}

This thesis might serve as a manual for various occupational groups. For usability practitioners, it provides the crucial methodological steps and design principles necessary to develop a website for the efficient and effective user-driven communication of infection control guidelines. For infection control professionals, 
our research generated a comprehensive set of factors affecting the usability of infection control guidelines as a communication means and successful implementation of technology into daily work practice. This section presents the specific recommendations that emerged from this thesis's research, separated for design and implementation.

\subsubsection{Design recommendations (for usability practitioners)}

In Table 8.1., we synthesized the design principles presented in Chapters 3 to 6 . The consistency of findings obtained in multiple studies through different instruments accounts for the importance of three following design issues (Greene \& Caracelli, 1997):

1) Combine several search strategies to facilitate HCWs' individual search strategies when searching for infection control guidelines;

2) Choose a question-oriented approach for the communication of infection control guidelines: answer questions from daily work practice, in order to dovetail with HCWs' tacit knowledge;

3) Explicate the relationship between user-driven guidelines provided by the website and guidelines locally enforced.

Taking these principles into account when designing communication of infection control guidelines will facilitate efficiency (better navigation, findability, and lower extraneous cognitive load), enhance information quality (effectiveness), and lead to a higher degree of satisfaction among HCWs, since the guidelines match their current needs and knowledge base.

One could argue that these design recommendations do not substantially differ from standard usability heuristics, such as Nielsen's ten general principles for user interface design (Nielsen, 1994). One of these principles, for instance, is "flexibility and efficiency of use", implying that accelerators should be incorporated so that the system can cater to both inexperienced and experienced users. This heuristic, however, is a general rule of thumb rather than a specific design recommendation (Nielsen, 1994). Our design issues can be seen as usability heuristics translated into context-driven design recommendations for the communication of infection control guidelines.

\subsubsection{Implementation recommendations (for infection control professionals)}

Chapters 6 and 7 provided a comprehensive set of barriers and facilitators for website adoption and guideline adherence, respectively. In both contexts, the organization's safety climate appeared to be a paramount reinforcer. The two studies showed that 
individual (predisposing) and work-related (enabling) facilitators provide the initial motivation for website adoption and guideline adherence, but a supportive safety climate is necessary to allow this motivation to be realized.

Because safety climate appeared to be positively affecting both website adoption as well as guideline adherence, we suggest addressing both behaviors in a bilateral intervention in which the website with infection control guidelines can be deployed to improve guideline adherence (Bates \& Gawande, 2003; Verhoeven et al., 2009e). Websites like the one developed can improve communication, make evidence-based guidelines more accessible, and assist with clinical decision making (Thomas et al., 1999). In other words, the website can provide targeted, evidence-based medical information to meet the specific needs of a caregiver as part of the care process, which is called Information Therapy (Gwinn \& Seidman, 2007).

The basis for such a bilateral intervention should be training/feedback, since training/ feedback emerged as the most salient reinforcing facilitator for behavior change, as was reported in Chapters 6 and 7. In the training program, factors from all three PRECEDE categories should be addressed. Table 8.2. presents the components to be incorporated into a training program aimed at improving safety behavior. Integration of all PRECEDE categories in a training program enables one intervention strategy to reach multi-faceted results. For instance, when individual HCWs are confronted with their non-adherent behavior recorded by hidden video cameras during a training session, individual attitudes toward guideline adherence will be favorably predisposed. Or, when website use is incorporated in training sessions and HCWs are enabled to try out the innovation without total commitment and with minimal investment, the prospects for successful adoption improve. In sum, the training program should comprise several components that mutually reinforce each other. Also, training should no longer be content-driven but rather be action oriented (Van Gemert-Pijnen, 2003). Multi-faceted interventions have more chance of success than do single-faceted approaches or promotion programs focusing only on one or two elements (Pittet, 2000).

Once the training program has ended, the safety climate should be perpetuated by the integration of the website into the workflow. For example, the website may be incorporated into the hospital information system, and the guidelines from the website may function as a set of standardized orders, with detailed, explicit instructions based on dynamic patient-specific parameters, available at the point-ofcare in specific clinical situations by means of a reminder system (Phansalkar et al., 2008). 
Table 8.1 Design principles for a user-driven website with infection control guidelines: synthesized results from Chapters 2 to 6

\begin{tabular}{|c|c|c|c|c|c|c|}
\hline \multirow[b]{2}{*}{ Presentation } & \multirow{2}{*}{$\begin{array}{l}\text { Design principle } \\
\text { Include visual aids such as tables, tree diagrams, and step-by-step plans }\end{array}$} & \multicolumn{5}{|c|}{ Chapter } \\
\hline & & 2 & & 4 & & 6 \\
\hline & Use soft colors & & 3 & & 5 & 6 \\
\hline & Insert pictures & & & & 5 & 6 \\
\hline & Prevent inclusion of too much running text & & & & & 6 \\
\hline & Do not include advertisements & & & & & 6 \\
\hline & Keep the design simple without unnecessary frills & & & & & 6 \\
\hline & Include headings whenever appropriate & & & & & 6 \\
\hline & Keep font size not too small & & & & & 6 \\
\hline \multirow[t]{6}{*}{ Structure } & $\begin{array}{l}\text { Categorize guidelines according to health care workers' mental models: } 1 . \text { MRSA in general; } \\
\text { 2. Screening; 3. Protective measures; } 4 \text {. Disinfection measures; } 5 . \text { Admission; } 6 \text {. Transport; } 7 \text {. } \\
\text { Discharge; 8. Treatment; } 9 \text {. MRSA and your own health; 10. Communication }\end{array}$ & 2 & 3 & & & \\
\hline & $\begin{array}{l}\text { Present guidelines according to a standardized format: title, short answer, comments, sources, } \\
\text { and multimedia examples }\end{array}$ & & 3 & 4 & & \\
\hline & $\begin{array}{l}\text { Combine several search strategies to facilitate health care workers' individual search strategies: } \\
\text { search engine, frequently asked questions, and categories }\end{array}$ & & 3 & 4 & 5 & 6 \\
\hline & $\begin{array}{l}\text { Include a breadcrumb trail to help health care workers keep track of their location within the } \\
\text { system }\end{array}$ & & 3 & 4 & & 6 \\
\hline & Make things clickable, when relevant & & & & & 6 \\
\hline & Avoid Portable Document Formats (PDF) & & & & & 6 \\
\hline \multirow[t]{11}{*}{ Content } & Formulate guidelines directly, imperatively, and concisely & 2 & & & 5 & \\
\hline & Provide background information about the disease & 2 & & & & 6 \\
\hline & Provide summary (maximum of 1 page $\mathrm{A}_{4}$ ) & 2 & & & & \\
\hline & Provide tools for application of the guidelines & 2 & & & & \\
\hline & Include information about education & 2 & & & & 6 \\
\hline & Use health care workers' terminology and avoid difficult vocabulary & 2 & & 4 & & \\
\hline & Motivate guidelines by mentioning dangers and risks & 2 & & & & 6 \\
\hline & Include clinical advantages of adherence & 2 & & & & 6 \\
\hline & Provide all necessary parts, elements, or steps for making a decision for practice & 2 & & & 5 & 6 \\
\hline & Provide additional, practical information next to basic information. & & & & & 6 \\
\hline & Choose a question-oriented approach: answer questions from daily work practice & 2 & & 4 & 5 & 6 \\
\hline \multirow[t]{12}{*}{ Quality } & Provide (levels of) evidence on which the guidelines are based & 2 & & & 5 & 6 \\
\hline & Provide risk categories & 2 & & & & 6 \\
\hline & Include information about cost-effectiveness & 2 & & & & \\
\hline & $\begin{array}{l}\text { Include references to other guidelines, law, sources for more information, appendices, and } \\
\text { forms }\end{array}$ & 2 & & & 5 & 6 \\
\hline & Add date of revision & 2 & & 4 & & 6 \\
\hline & Mention the frequency with which guidelines are updated & & & & 5 & 6 \\
\hline & $\begin{array}{l}\text { Ensure that guidelines communicated on the website correspond with health care workers' } \\
\text { work practice }\end{array}$ & & & & 5 & 6 \\
\hline & Make explicit that presented guidelines might differ from guidelines locally enforced & & & & 5 & \\
\hline & Disclose guideline authors & 2 & & 4 & 5 & 6 \\
\hline & Disclose who approved the guidelines & 2 & & & & 6 \\
\hline & Include contact information & 2 & & & & \\
\hline & Ensure the website is among the top three hits in Google & & & & 5 & 6 \\
\hline
\end{tabular}


Table 8.2 Intervention strategies to enhance the adoption of a website with infection control guidelines and guideline adherence, as suggested by health care workers $(\mathrm{N}=44)$

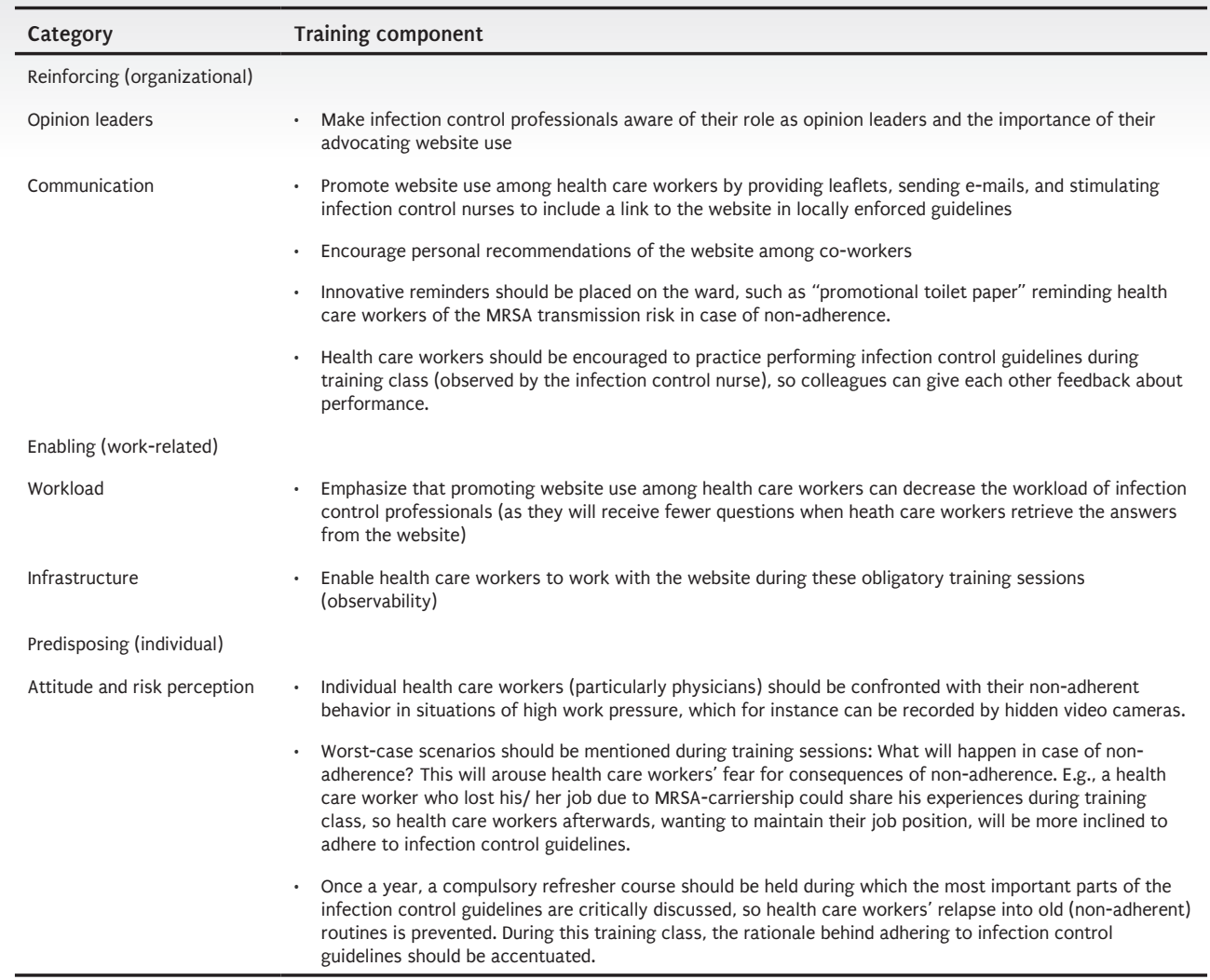




\subsection{Directions for future research}

The website developed in this thesis's research was a first attempt towards the design of user-driven communication of infection control guidelines. Although the website was highly valued among its users, there is always room for improvement. Several aspects regarding both the quality of the documentation as well as the context of use (see Chapter 1, section 1.1.3) were not addressed during our studies. These issues serve as input for future research.

\subsubsection{Quality of documentation}

The website that we developed only enables static information retrieval. A possible next step that may be realized in the short term is to provide web-based tailored guidelines. HCWs seem to prefer information explaining treatments, diseases, and measurements related to their own specific situation (Cawsey et al., 2000; Harbarth \& Emonet, 2006). Moreover, studies in health promotion suggest that information has a higher impact when it is adapted to the individual situation (Garg et al., 2005; Kawamoto et al., 2005; Peleg et al., 2009; Thomas et al., 1999; Thursky \& Mahemoff, 2007). Tailored guidelines that are adapted to the individual HCW at the level of sentences, phrases, and even words avoid the need to search through the copious guideline documentation available. An illustrative application is the realtime microbiology browser and decision support tool for antibiotic prescribing, developed by Thursky and Mahemoff (2007). The rules engine uses microbiology data and information entered by the user (allergy type, weight) to generate antibiotic recommendations for the treatment of an isolate. Each recommendation contains general educational information about the organism, then specific recommendations based on site (for example, the removal of catheters with blood isolates), registered patient allergies, and antibiotic susceptibility profiles. In the longer run, research might concentrate on the development of more advanced systems. Web 2.0 offers tremendous possibilities for the expansion of the website's functionalities, such as the development of personalized applications (Giustini,2006). A personalized system can alter aspects of its structure, functionality or interface on the basis of a user model generated from implicit and/or explicit user input, in order to accommodate the differing needs of individuals or groups of individuals and their changing needs over time (Van Velsen et al., 2008). This implies that not only the content of the information (as is the case with tailored information) but also its structure and presentation will change according to the user's preferences.

Tailored web-based guidelines that are provided to HCWs at the time they actually need them correspond to the Information Therapy concept, which is advocated by the Institute of Medicine to improve the quality of care (Gwinn \& Seidman, 2007). Expressions such as "just-in-time knowledge" or "delivering the right knowledge at the right time" are often used to describe applications within the Information Therapy approach (Cole et al., 1997).

Future research should focus on the possibilities of incorporation of "just-in-time" knowledge into daily work practice in health care. Since the EUREGIO MRSA-net 
Twente/Münsterland (see Chapter 1, section 1.1.1.) will be continued from October 1, 2009 under the name of EURSAFETY Health Net, the website will serve as a basis for the development of a web-based disease management system. This system will offer tailored information to HCWs concerning the prevention and control of MRSA, but also other infectious diseases. Several options exist to provide these guidelines to HCWs. First, we could develop an interactive dialogue functionality. Since users are often unable to express their need for information in a single, self-contained query within the medical domain (Nijland et al., 2008), the dialogue system will allow users to ask for clarification, make corrections to the system's interpretation of utterances, and ask follow-up questions (comparable to the system developed by Chai et al., 2001). Second, the design of an e-learning module is an interesting possibility. Since an efficient format of instructional materials promotes learning (Clark et al., 2006), it may be interesting to develop an e-learning program aimed at increasing HCWs' knowledge regarding infection prevention and control. In industries such as aviation and nuclear power plants, e-learning courses have proven to be effective in motivating employees to work safely (Bates et al., 2003). Since the same user-driven design process will underlie the development of the web-based disease management system, the results of the needs analysis will determine on which functionality we will concentrate.

\subsubsection{Context of use}

Throughout this thesis, we advocated considering the role of the contextual environment in which the application will be used. We recommended a multifaceted approach and suggested several intervention strategies for website implementation. Evaluation of these strategies should be subjects of future research. Also, the following issues regarding the context of use might be addressed in future research:

Cues in the environment may contribute significantly to the improvement of guideline adherence among HCWs. For instance, to encourage HCWs' ownership of the intervention, Pittet's “Talking Walls" model may be used. The principle of “Talking Walls" is to harness art and humor to reinforce the principles of infection prevention through improved hand hygiene among HCWs. Staff from different wards could be invited to help design a poster featuring their own hand hygiene message, which can then be captured by an artist brought in to work on the project. The resulting posters would then be placed throughout the hospital, acknowledging the ward's creativity. A key feature of "Talking Walls" is the active involvement of individual HCWs and the hospital management in the activities for promoting infection prevention, which was advocated in this thesis as well. This factor has probably been essential for the long-term success of "Talking Walls", since posters alone are not very effective (Pittet \& Boyce, 2001). The process of HCWs jointly creating objectives for behavior change and articulate solutions might create a collective awareness towards the importance of guideline adherence. In future research, the effectiveness of initiatives such as "Talking Walls" should be evaluated.

Although the website appeared to be successful for the efficient and effective retrieval of infection control guidelines for HCWs, it is of interest to verify whether the format of action oriented guideline communication also works for public health 
education. MRSA is ever more becoming a public health problem, due to its prevalence among cattle, raw meat, and otherwise healthy people (Van Loo et al., 2007; Voss et al., 2005). Therefore, it is essential that risk communication promote awareness and recognition of MRSA among the general public so they can adopt preventive health and infection control measures. In order to do so, a website for the Dutch general public was developed (Verhoeven et al., 2009b). This website informs patients and their relatives of their health conditions and thus promotes active participation in their health management and empowering them to perform better self-care by providing easy access to useful, up-to-date information about how to cope with MRSA in daily practice. Future research should concentrate on the role this website can have in reducing anxiety towards MRSA among the general public.

Although this research project emphasized the benefits of user-driven design, the project was very time and cost intensive. In practice, it is not always feasible to perform such an extensive user-driven design process that requires the involvement of a wide range of end-users and stakeholders (Abras et al., 2004). Therefore, a "discount usability engineering version" of the methodology was developed and applied to an innovation project in Dutch nursing homes in the Twente region, providing input for a first working prototype of a website with infection control guidelines (the total cost was 7500 euros). Our "discount usability engineering method" was comprised of similar methods to that developed by Nielsen (1989), since we also employed prototyping and thinking-aloud tests. The main difference is that we preferred to perform the Card Sort Task instead of heuristic evaluation. Future research should validate discount usability engineering for the design of guideline communication.

\subsection{In short}

This study of the development, implementation, and evaluation of a website with infection control guidelines can be seen as an example of research into design communication in practice. It explores how a design process can be structured and administered in order to maximize the chances of successful communication taking place (Schellens et al., 2001).

More specifically, this thesis demonstrated that a shift in the function of guidelines from regulation towards communication of safe work practices leads to more effective and efficient guideline retrieval among HCWs. In order to tailor the communication of guidelines to the target group's tacit knowledge, it is crucial to involve intended users (HCWs) in the design process. However, experts (infection control professionals) should see to the maintenance of the evidence-based, medical content. Next, it is crucial to consider organizational factors when implementing the user-driven guideline communication and to take into account value differences between occupational groups.

This thesis provides the methodological steps and design principles necessary to make clinical practice guidelines more accessible to HCWs and suggests how to optimally implement guideline communication in daily work practice. 
References 



\section{References}

Abras C, Maloney-Krichmar D, Preece J. User-Centered Design. In Bainbridge W. Encyclopedia of Human-Computer Interaction. Thousand Oaks, Sage Publications, 2004 .

Avery $\mathrm{CH}$, March J, Brook RH. An assessment of the adequacy of self-care by adult asthmatics. J Community Health 1980;5:167-80.

Bartholomew LK, Parcel GS, Kok G. Intervention Mapping: A Process for Developing Theory and Evidence-Based Health Education Programs. Health Educ Behav $1998 ; 25: 545-63$.

Bates DW, Gawande AA. Improving safety with information technology. N Engl J Med 2003; 348:25 26-34.

Bennett NL, Casebeer LL, Kristofco RE, Strasser SM. Physicians' internet informationseeking behaviors. J Cont Educ Health Prof 2004;24:31-8.

Berhe M, Edmond MB, Mearman GM. Practices and an assessment of health care workers' perceptions of compliance with infection control knowledge of nosocomial infections. Am J Infect Control 2005;33:55-7.

Bero LA, Grilli R, Grimshaw J, Harvey E, Oxman AD, Thomson MA. Closing the gap between research and practice : an overview of systematic reviews of interventions to promote the implementation of research findings. BMJ 1998;317:465-8.

Bettcher D, Lee K. Globalisation and public health. J Epidemiol Community Health 2002;56:8-17.

Beyer H, Holtzblatt K. Contextual Design: Defining Customer-Centered Systems. San Francisco, Morgan Kaufmann, 1998.

Berg M. Rationalizing medical work: decision-support techniques. Cambridge, MIT Press, 1997.

Boulos MNK. Map of dermatology: 'first-impression' user feedback and agenda for further development, Health Info Libr J 2006;23:203-13

Boren MT, Ramey J. Thinking aloud: Reconciling theory and practice. IEEE Trans Prof Comm 2000;43:261-78.

Bosua R, Scheepers R. Towards a model to explain knowledge sharing in complex organizational environments. Know Mngt Res Practice 2007:5:93-109.

Cain M, Mittman R. Diffusion of innovation in health care. California Health Care Foundation, Oakland, 2002. 
Carey TS, Garett J. Patterns of Ordering Diagnostic Tests for Patients with Acute Low Back Pain. Ann Intern Med 1996;10:807-14.

Carroll JM. Encountering others: Reciprocal openings in participatory design and usercentered design. Hum Comput Interact 1996;1 1:285-90.

Casebeer L, Bennett N, Kristofco R, Carillo R, Centor R. Physician Internet Medical Information Seeking and Online Continuing Education Use Patterns. J Contin Educ Health Prof 2002;22:33-42.

Cawsey A, Jones RB, Pearson J. The Evaluation of a Personalised Information System for Patients with Cancer. UMUAI 2000;10:47-72.

Centers for Disease Control and Prevention. Healthcare-Associated Infections (HAIs). Atlanta, Centers for Disease Control, Department of Health and Human Services, 2009. URL: http://www.cdc.gov/ncidod/dhqp/healthdis.html [accessed: 2009 Apr 13]

Centers for Disease Control and Prevention (a). Information About MRSA for Healthcare Personnel. Atlanta, Centers for Disease Control, Department of Health and Human Services, 2009. URL: http://www.cdc.gov/ncidod/dhqp/ar_mrsa_healthcareFS.html [accessed: 2007 March 29].

Centers for Disease Control and Prevention (b). Community-Associated MRSA Information for the Public. Atlanta, Centers for Disease Control, Department of Health and Human Services, 2009. URL: http://www.cdc.gov/ncidod/dhqp/ar_mrsa_ca_public. html [accessed: 2007 March 29]

Centers for Disease Control and Prevention (c). Community-Associated MRSA Information for Clinicians. Atlanta, Centers for Disease Control, Department of Health and Human Services, 2009. URL: http://www.cdc.gov/ncidod/dhqp/ar_mrsa_ca_ clinicians.html [accessed: 2007 March 29]

Chai J, Budzikowska M, Horvath V, Nicolov N, Kambhatla N, Zadrozny W. Natural Language SalesAssistant - A Web-based Dialog System for Online Sales. In: Proceedings of the Thirteenth Innovative Application of Artificial Intelligence Conference. AAAI Press, Seattle, 2001, 19-26.

Clark R, Nguyen F, Sweller J. Efficiency in Learning: Evidence-Based Guidelines to Manage Cognitive Load. San Francisco, Pfeiffer, 2006.

Cohen, J. A coefficient of agreement for nominal scales. Educ Psycho Meat 1960;20:3746.

Coia JE, Duckworth GJ, Edwards DI, Farrington M, Fry C, Humphreys H, Mallaghan C, Tucker DR, for the Joint Working Party of the British Society of Antimicrobial Chemotherapy, The Hospital Infection Society \& the Infection Control Nurses Association Journal of Hospital Infection. Guidelines for the control and prevention of methicillin-resistant Staphylococcus aureus (MRSA) in healthcare facilities.J Hosp Infect 2006;66:1-44. 
Cole K, Fischer O, Saltzman P. Just-in-Time Knowledge Delivery. A case study of an award-winning support system demonstrates the vital characteristics and primary design goals for generating peak performance. Commun ACM 1997;14:924-7.

Cooper D, Farmery K, Johnson M, Harper C, Clarke FL, Holton P, Wilson S, Rayson $\mathrm{P}$, Bence $\mathrm{H}$. Changing personnel behavior to promote quality care practices in an intensive care unit. Ther Clin Risk Manag 2005;1:321-32.

Coulter A. Evidence based patient information is important, so there needs to be a national strategy to ensure it. BMJ 1998;317:225-6.

Currim IS, Gurbaxani V, LaBelle J, Lim J. Perceptual structure of the desired functionality of internet-based health information systems. Health Care Man Sci 2006;9:15 1-170.

DeJoy DM, Searcy C, Murphy LR, Gershon R. Behavioral-diagnostic analysis of compliance with universal precautions among nurses.J Occup Health Psychol 2000;5:127-41.

Deurenberg RH, Vink C, Oudhuis GJ, Mooij J, Driessen C, Coppens G, et al. Different clonal complexes of Methicillin-Resistant Staphylococcus aureus are disseminated in the Euregio Meuse-Rhine region. Antimicrob Agents Chemother 2005;10:4263-71.

Duckworth G, Heathcock, R. Guidelines on the control of methicillin-resistant Staphylococcus aureus in the community.J Hosp Infect 1995;21:1-12.

Dutch Working party on Infection Prevention (a). MRSA in nursing homes. Dutch Working Party on Infection Prevention, Leiden, 2005.

Dutch Working party on Infection Prevention (b). MRSA- general, hospitals. Dutch Working Party on Infection Prevention, Leiden, 2005.

Eastin MS. Credibility Assessments of Online Health Information: The Effects of Source Expertise and Knowledge of Content. JCMC 2001;6.

Elling MGM. Veiligheidsvoorschriften in de industrie. Een verkenning van problemen en mogelijkheden. Enschede, Universiteit Twente, 1991.

Ericsson K, Simon H. Protocol Analysis: Verbal Reports as Dat. Boston, MIT Press, 1993.

Estabrooks C, O'Leary K, Ricker K, Humphrey C. The internet and access to evidence: how are nurses positioned? J Adv Nurs 2003;42:73-81.

Eysenbach G, Powell J, Kuss O, Eun-Ryoung S. Empirical Studies Assessing the Quality of Health Information for Consumers on the World Wide Web. A Systematic Review. JAMA 2002;287:2691-700.

Fetters MD, Ivankova NV, Ruffin MT, Creswell JW, Power D. Developing a Web site in primary care. Fam Med 2004;36:65 1-9.

Ferney SL, Marshall AL. Website physical activity interventions: preferences of 
potential users. Health Educ Res 2006;21:560-6.

Fervers B, Burgers JS, Haugh MC, Brouwers M, Browman G, Cluzeau F, Philip T. Predictors of high quality clinical practice guidelines: examples in oncology. Int J Qual Health Care 2005;17:123-32.

Francke AL, Smit MC, Veer AJE de, Mistiaen P. Factors influencing the implementation of clinical guidelines for health care professionals: a meta-review. BMC Med Inform Decis Mak 2008;38.

Friedrich AW, Daniels-Haardt I, Sonntag AK, Verhoeven F, Gemert-Pijnen L, Mellman A, Hendrix R. EUREGIO MRSA-net Twente/Münsterland. Westfälisches Ärzteblatt 2006;9:5 1-2.

Friedrich AW, Daniels-Haardt I, Gemert-Pijnen JEWC van, Hendrix MGR, Eiff C von, Kipp F, Becker K, Köck R, Verhoeven F, Mellman A. Ein regionales Netzwerk zur Prävention und Kontrolle von Infektionen durch MRSA: EUREGIO MRSA-net Twente/Münsterland. Epidem Bull 2007,33;307-11.

Friedrich AW, Daniels-Haardt I, Köck R, Verhoeven F, Mellmann A, Harmsen D, GemertPijnen JEWC van, Becker K, Hendrix MGR. EUREGIO MRSA-net Twente/Münsterland- A Dutch-German cross-border network for the prevention and control of infections caused by Methicillin-resistant Staphylococcus aureus. Euro Surveill 2008;35:1-1 1.

Frøkjær E, Hertzum M, Hornbæk K. Measuring usability: are effectiveness, efficiency, and satisfaction really correlated? In: Proceedings of ACM Conference on Human Factors in Computer Systems. ACM Press, New York, 2000, 345-52.

Foundation of Nursing Studies. Taking Action. Foundation of Nursing Studies, London, 1998.

Gagne RM. The Conditions of Learning and Theory of Instruction. New York, Holt Rinehart Winston, 1985.

Garg AX, Adhikari NK, McDonald H, Rosas-Arellano MP, Deveraux PJ, Beyene J, Sam J, Haynes RB. Effects of computerized clinical decision support systems on practitioner performance and patient outcomes: a systematic review. JAMA 2005;293:1223-38.

Gemert-Pijnen JEWC van. The development and functioning of infection prevention protocols. A study of communication driven by legislation and regulations. Enschede, University of Twente, 2003.

Gemert-Pijnen JEWC van, Hendrix MGR, Palen J van der, Schellens PJ. Performance of methicillin-resistant Staphylococcus aureus protocols in Dutch hospitals. Am J Infect Control 2005;33,377-84.

Gemert-Pijnen JEWC van, Hendrix MGR, Palen J van der, Schellens PJ. Effectiveness of protocols for preventing occupational exposure to blood and body fluids in Dutch hospitals. J Hosp Infect 2006;62:166-73. 
Gershon RR, Karkashian CD, Grosch JW, Murphy LR, Escamilla-Cejudo A, Flanagan PA, Bernacki E, Kasting C, Martin L. Hospital safety climate and its relationship with safe work practices and workplace exposure incidents. Am J Infect Control 2000;28:21 1-21.

Giustini D. How Web 2.0 is changing medicine. BMJ 2006;333:1283-4.

Glasgow RE, Funnell MM, Bonomi AE, Davis C, Beckham V, Wagner EH. Selfmanagement aspects of the improving chronic illness care breakthrough series: implementation with diabetes and heart failure teams. Ann Behav Med 2002;2:80-7.

Griffiths KM, Christensen H. Website Quality Indicators for Consumers. J Med Internet Res 2005;7:e55.

Gould IM. Costs of hospital-acquired methicillin-resistant Staphylococcus aureus (MRSA) and its control. Int J Antimicrob Agents 2006;28:379-84.

Gould JD, Lewis C. Designing for usability: key principles and what designers think. Commun ACM 1985;28:300-11.

Gosling AS, Westbrook J, Coiera EW. Variation in the use of online clinical evidence: a qualitative analysis. Int J Med Inform 2003;69:1-16.

Green LW, Kreuter MW. Health Promotion Planning; an Educational and Environmental Approach. Palo Alto, Mayfield PublishingCo, 2006.

Greene JC, Caracelli VJ. (eds.). Advances in mixed-method evaluation: The challenges and benefits of integrating diverse paradigms. San Francisco, Jossey-Bass, 1997.

Greenhalgh T, Robert R, Macfarlane F, Bate P, Kyriakidou O. Diffusion of Innovations in Service Organizations: Systematic Review and Recommendations. Milbank $Q$ 2004;82:581-629.

Grimshaw JM, Russell IT. Effect of clinical guidelines on medical practice: a systematic review of rigorous evaluations. Lancet 1993;8883:1317-22.

Grol R. Successes and failures in the implementation of evidence-based guidelines for clinical practice. Med Care 2001;39:46-54.

Grol R, Wensing M. What drives change? Barriers to and incentives for achieving evidence-based practice. Med J Aus 2004;15:57-60.

Gross R, Greenfield S, Cretin S, Ferguson J, Grimshaw J, Grol R. Optimal methods for guideline implementation. Med Care 2001;39:46-54.

Guldenmund FW. The nature of safety culture: a review of theory and research. Saf Sci 2000;34:215-57.

Gwinn BR, Seidman J. The Ix Evidence Base: Using Information Therapy to Cross the Quality Chasm. Bethesda, Center for Information Therapy, 2007.

Hayward RSA, Wilson MC, Tunis SR, Bass EB, Guyatt G. Users' guides to the Medical 
Literature. VIII. How to Use Clinical Practice Guidelines. Are the Recommendations Valid? JAMA 1995;274, 570-4.

Haak MJ van den. A penny for your thoughts - investigating the validity and reliability of think-aloud protocols for usability testing. University of Twente, Enschede, 2008.

Hamilton M. Message variables that mediate and moderate the effect of equivocal language on source credibility. J Lang Soc Psychol 1998;17:109-43.

Harbarth S, Emonet S. Navigating the World Wide Web in search of resources on antimicrobial resistance. Clin Infect Dis 2006;43:72-8.

Harbarth S, Pittet D. Methicillin-resistant Staphylococcus aureus. Lancet Infect Dis $2005 ; 5: 653-63$.

Health First Europe. Healthcare Associated Infections cost Europe $\$ 5.5$ billion per year. Brussels, Health First Europe, 2009. URL: http://www.healthfirsteurope.org/index. php?pid=91\&id=99 [accessed: 2009 Apr 13]

Health On the Net Foundation. HON code on conduct (HONCode) for medical and health web sites. Geneva, Health On the Net Foundation, 2009. URL: http://www.hon. ch/HONcode/ [accessed: 2009 Apr 23]

Hedin K, Babic A, Frydén A. Take care: guidelines for patients with chronic hepatitis C. Stud Health Technol Inform 1999;68:783-8.

Hersh WR, Crabtree MK, Hickam DH, Sacherek L, Friedman CP, Tidmarsh P, Mosbaek C, Kraemer D. Factors associated with success in searching MEDLINE and applying evidence to answer clinical questions. JAMIA 2002;9:283-93.

Ho MY, Hovenga EJS. What Do Nurses Have to Say About Information Technology in Their Workplace? In: HIC Proceedings. Health Informatics Society of Australia, Brunswick East, 1999.

Hofstede G. Culture and Organisations: Software of the Mind. London, Harper Collins Publishers, 1994.

Holtzblatt K, Wendel JB, Wood S. Rapid Contextual Design: A How-to guide to key techniques for user-centered design. San Francisco, Morgan-Kaufmann, 2005.

Holzinger A. Usability Engineering for Software Developers. Commun ACM 2005;48:7174 .

Holzinger A, Kickmeier-Rust M, Wassertheurer S, Hessinger M. Learning Performance with Interactive Simulations in Medical education: Lessons learned from results of learning complex physiological models with the HAEMOdynamics SIMulator. Comput Edu 2009;5 2:292-301.

Hom J. The Usability Methods Toolbox. San José State University, San José, 1998. URL: http://jthom.best.vwh.net/usability/ [accessed: 2009 Apr 26] 
Hornbæk K. Current practice in measuring usability: Challenges to usability studies and research. Int J Hum-Comput Stud 2006;64:79-102.

Humphreys $\mathrm{H}$. Implementing guidelines for the control and prevention of Methicillinresistant Staphylococcus Aureus and vancomycin-resistant enterococci: how valid are international comparisons of success? J Hosp Infect 2006;62:1 13-35.

International Organization for Standardization. ISO 9241-11 Ergonomic requirements for office work with visual display terminals (VDTs) -- Part 11: Guidance on usability. Geneva, Switzerland, 1998.

International Organization for Standardization. Human-centered design processes for interactive systems' from ISO 13407. Geneva, Switzerland, 1999.

Jaspers MWM. A Comparison of usability methods for testing interactive health technologies: Methodological aspects and empirical evidence. Int J Med Inform 2009;78: 340-53.

Jeannot J, Scherer F, Pittet V, Burnand B, Vader J. Use of the World Wide Web to Implement Clinical Practice Guidelines: A Feasibility Study.J Med Internet Res 2003:5:e12.

Johnson CM, Johnson TR, Zhang J. A user-centered framework for redesigning health care interfaces. J Biomed Inform 2005;38:75-87.

Jong $M$ de, Lentz L. Scenario evaluation of municipal websites. Development and use of an expert-focused evaluation tool. Gov't Info Q 2006;23:191-206.

Jong M de, Schellens PJ. Toward a document evaluation methodology. What does research tell us about the validity and reliability of evaluation methods? IEEE Trans Prof Comm 2000;43:242-60.

Kaplan B. Addressing Organizational Issues into the Evaluation of Medical Systems.J Am Med Inform Assoc 1997:4:94-101.

Kawamoto K, Houlihan CA, Balas EA, Lobach DF. Improving clinical practice using clinical decision support systems: a systematic review of trials to identify features critical to success. BMJ 2005; 330:765.

Keulers BJ, Keulers MJ, Scheltinga MRM. Evidence-based development of interactive patient education programs: A guide forachieving optimal computer-based patient education. Eur J Plast Surg 2006;29:169-175.

Kermode M, Jolley D, Langkham B, Thomas M, Holmes W, Gifford S. Compliance with Universal/Standard Precautions among health care workers in rural north India. Am J Infect Control 2003;33:27-33.

Kerr C, Murray E, Stevenson F, Gore C, Nazareth. Internet interventions for long- term conditions: patient and caregiver quality criteria.J Med Internet Res 2006;8:e13. 
Kinzie M, Cohn W, Julian M, Knaus WA. A user-centered model of website design: needs assessment, user interface design and rapid prototyping. J Am Med Inform Assoc 2002;9:320-30.

Klevens RM, Edwards JR, Richards CL Jr, Horan TC, Gaynes RP, Pollock DA, Cardo DM. Estimating health care-associated infections and deaths in U.S. hospitals, 2002. Public Health Rep 2007;122:160-6.

Kok G, Schaalma H, Ruiter RA, van Empelen P, Brug J. Intervention mapping: protocol for applying health psychology theory to prevention programmes.J Health Psychol 2004;9:85-98.

Kolbe J, Vamos M, James F, Elkind G, Garrett J. Assessment of Practical Knowledge of Self-Management of Acute Asthma. Chest 1996;109:86-90.

Koyani SJ, Bailey RW, Nall JR. Research-Based Web Design \& Usability Guidelines. Washington DC, U.S. Government Printing Office, 2006.

Kukafka R, Johnson JB, Linfante A, Allegrante JP. Grounding a new information technology implementation framework in behavioral science: a systematic analysis of the literature on IT use. J Biomed Inform 2003;36:218-27.

Lachin JM, Matts JP, Wei LJ. Randomization in Clinical Trials: Conclusions and Recommendations. Con Clin Trial 1988;9:365-74.

Landis JR, Koch GG. The measurement of observer agreement for categorical data. Biometrics 1977;33:159-174.

Leonard D, Sensiper S. The role of tacit knowledge in group innovation. Calif Manag Rev 1998;40:112-132.

Li JP, Kishore R. How robust is the UTAUT instrument?: a multigroup invariance analysis in the context of acceptance and use of online community weblog systems. In Proceedings ACM SIGMIS CPR. Claremont, ACM, 2006.

Lohr KN, Field MJ. A provisional instrument for assessing clinical practice guidelines. In: Field MJ, Lohr KN (eds). Guidelines for clinical practice. From development to use. Washington D.C., National Academy Press, 1992.

Loo I van, Diederen BMW, Savelkoul PHM, Woudenberg JHC, Roosendaal R, Belkum A van, Lemmens-den Toom N, Verhulst C, Keulen PHJ van, Kluytmans JAJW. MethicillinResistant Staphylococcus aureus in Meat Products, the Netherlands. Emerg Infect Dis 2007;13:1753-5.

Loorbach N, Karreman J, Steehouder M. Adding Motivational Elements to an Instruction Manual for Seniors: Effects on Usability and Motivation. Tech Commun 2007:54:34358.

Lundstrom T, Pugliese G, Bartley J, Cox J, Guither C. Organizational and environmental factors that affect worker health and safety and patient outcomes. Am J Infect Control 
2002;30:93-106.

Lynch PJ, Horton S. Yale Style Manual. Yale University Center for Advanced Instructional Media, New Haven, 1997. URL: http://info.med.yale.edu/caim/manual. [accessed: 2009 Apr 21]

Madle G, Kostkova P, Mani-Saada J, Weinberg J, Williams P. Changing public attitudes to antibiotic prescribing: can the Internet help? Inform Prim Care 2004;12:19-26.

Marchionini G. Information seeking in electronic environments. Cambridge, Cambridge University Press, 1995.

Marcus A, Gould EW. Crosscurrents: cultural dimensions and global Web user-interface design. Interactions 2000;7:32-46.

McCaughan D, Thompson CA, Cullum NA, Sheldon TA, Raynor P. Nurse practitioner and practice nurses' use of research information in clinical decision making: qualitative findings from a national study. Fam Pract 2005;22:490-7.

McCoy KD, Beekmann SE, Ferguson KJ, Vaughn TE, Torner JC, Woolson RF, Doebbeling BN. Monitoring adherence to standard precautions. Am J Infect Control 2001;29:24-31.

McDonald R, Waring J, Harrison S, et al. Rules and guidelines in clinical practice: a qualitative study in operating theatres of doctors" and nurses" views. Qual Saf Health Care $2005 ; 14: 290-294$.

McGovern PM, Vesley D, Kochevar L, Gershon RRM, Rhame FS, Anderson E. Factors affecting universal precautions compliance.J Bus Psychol 2000;15:149-61.

McRobb M. Writing Quality Manuals for ISO 9000 Series. United Kingdom, IFS Publications, 1990.

Miller M, Kearny N. Guidelines for clinical practice: development, dissemination and implementation Int J Nurs Stud 2004;41:813-21.

Morris $\mathrm{AH}$. Developing and implementing computerized guidelines for standardization of clinical decisions. Ann Int Med 2000;132:373-83.

Morris-Docker S, Tod A, Harrison JM, Wolstenholme D, Black R. Nurses' use of the Internet in clinical ward settings. J Adv Nurs 2004;48:157-66.

Mullen PD, Hersey JC, Iverson DC. Health behavior models compared. Soc Sci Med 1987;21:973-81.

Muller MJ. Participatory Design: The Third Space in HCl. In: Jacko J, Spears A. Handbook of $\mathrm{HCl}$. Lawrence Erlbaum, Hillsdale, 2002.

Murphy DM. From expert data collectors to interventionists: Changing the focus for infection control professionals. Am J Infect Control 2002;30:120-32.

Muto CA, Jernigan JA, Ostrowsky BE, Richet HM, Jarvis WR, Boyce JM, et al. SHEA 
guidelines for preventing nosocomial transmission of multidrug-resistant strains of Staphylococcus aureus and Enterococcus. Infect Control Hosp Epidemiol 2003;24:36286.

Nabitz U, Klazinga N, Walburg J. The EFQM excellence model: European and Dutch experiences with the EFQM approach in health care. IntJ Qual Health Care 2000;3:191201.

National Coordinator Infectious Disease Control. Draaiboek MRSA in de openbare gezondheidszorg. (Script MRSA in public health care). Bilthoven, National Coordinator Infectious Disease Control 2005.

Nielsen J. Usability engineering at a discount. In Salvendy G, Smith MJ (Eds.) Designing and Using Human-Computer Interfaces and Knowledge Based Systems. Amsterdam, Elsevier Science Publishers, 1989.

Nielsen J. Usability Engineering. San Francisco, Morgan Kaufmann, 1993.

Nielsen J. Heuristic evaluation. In Nielsen J, Mack RL. (Eds.) Usability Inspection Methods. New York, John Wiley \& Sons, 1994.

Nijland N, Van Gemert-Pijnen JEWC, Boer H, Steehouder MF, Seydel ER. Evaluation of Internet-based technology for supporting self-care: Problems encountered by patients and caregivers when using self-care applications. J Med Internet Res 2008;10:e13.

Norman DA. The Psychology of Everyday Things. New York, Basic Books, 1988.

Norman CD, Skinner HA. eHEALS: The eHealth Literacy Scale.J Med Internet Res 2006;8:e27.

Peters O. Social psychological determinants of mobile communication technology use and adoption. A comparison of three models to explain and predict mobile communication technology behavior. University of Twente, Enschede, 2007.

Parker D, Lawton R. Judging the use of clinical protocols by fellow professionals. Soc Sci Med 2000;5 1:669-77.

Patton MW. Qualitative evaluation and research methods. Newbury Park, Sage Publications, 1990.

Peleg M, Shachak A, Wang D, Karnieli E. Using multi-perspective methodologies to study users' interactions with the prototype front end of a guideline-based decision support system for diabetic foot care. Int J Med Inform 2009 (in press).

Phansalkar S, Weir CR, Morris AH, Warner HR. Clinicians' perceptions about use of computerized protocols: A multicenter study. Int J Med Inform 2008;77:184-193.

Pittet D. Infection control and quality health care in the new millennium. Am J Infect Control 2000;33:258-67. 
Pittet D, Boyce JM. Hand hygiene and patient care: pursuing the Semmelweis legacy. Lancet Infect Dis 2001;1:9-20.

Pittet D, Allegranzi B, Sax H, Bertinato L, Concia E, Cookson B, Fabry J, Richet H, Philip P, Spencer RC, Ganter BW, Lazzari S. Considerations for a WHO European strategy on health-care-associated infection, surveillance and control. Lancet Infect Dis 2005:5:242-50.

Pittet D, Donaldson L. Challenging the world: patient safety and health care-associated infection. Int J Qual Health Care 2006;1:4-8.

Pollock E, Chandler, P, Sweller J. Assimilating complex information. Learn Instruct 2002;12: 61-86.

Provost M, Koompalum D, Dong D, Martin BC. The initial development of the WebMedQual scale: domain assessment of the construct of quality of health web sites. Int J Med Inform 2006;75:42-57.

Rabon D, Chapman T. Persuasive Interviewing. Durham, Carolina Academic Press, 1994. Rasmussen J. Risk management in a dynamic society: a modelling problem. Safety Sci 1997;27:183-213.

Rippen H, Risk A. E-Health code of ethics. J Med Internet Res 2000;2:eg.

Robert Koch Institute. Vorwort und Einleitung der Kommission zur Richtlinie für Krankenhaushygiene und Infektionsprävention. (Foreword and Introduction from the Committee to the Guideline for Hospitalhygiene and Infection prevention) Bundesgesundheitsbl Gesundheitsforsch Gesundheitsschutz 2004;47:409-11.

Rogers EM. Diffusion of Innovations. New York, Free Press, 2003.

Rousseau N, McColl E, Newton J, Grimshaw J, Eccles M. Practice based, longitudinal, qualitative interview study of computerized evidence based guidelines in primary care. BMJ 2003;326:314.

Roy A, Kostkova P, Carson E, Catchpole M. Web-based provision of information on infectious diseases: a systems study. Health Inform J 2006;12,274-92.

Sachs, M. Successful strategies and methods of nursing standards implementation. Pflege 2006; 19:33-44.

Saillour-Glenisson F, Michel P. Individual and collective facilitators of and barriers to the use of clinical practice guidelines by physicians: a literature review. Rev Epidemiol Sante Publique 2003;51,65-80.

Sackett DL, Rosenberg WM, Gray JA, Haynes RB, Richardson WS. Evidence-based medicine: what it is and what it isn't. BMJ 1996;312: 71-2.

Scandurra I, Hägglund M, Koch S. From user needs to system specifications: Multi- 
disciplinary thematic seminars as a collaborative.J Biomed Inform 2008;41:557-69.

Schellens PJ, Klaassen R, Vries S de. Communicatiekundig ontwerpen. Methoden, perspectieven en toepassingen. Assen, Koninklijke Van Gorcum, 2001.

Scriven M. Beyond Formative and Summative Evaluation. In McLaughlin MW, Phillips, eds. Evaluation and Education: A Quarter Century. Chicago, University of Chicago Press, 1991.

Shekelle, PG, Kravitz RL, Beart J, Marger M, Wang M, Lee M. Are nonspecific practice guidelines potentially harmful? A randomized comparison of the effect of nonspecific versus specific guidelines on physician decision making. Health Serv Res 2000;34:142948.

Sinkowitz-Cochran RL, Stein GP, Keyserling HL, Levine GL, Jarvis W, The Pediatric Prevention Network. The Internet: A practical example of the use of new technology in the assessment of vancomycin use in pediatrics. Am J Infect Control 2000;28:459-64.

Smith G. Tagging: People-powered Metadata for the Social Web. Indianapolis, New Riders, 2007.

Smith TJ, Hillner BE. Ensuring quality cancer care by the use of clinical practice guidelines and critical pathways.J Clin Oncol 2001;19:2886-97.

Snyder C. Using Paper Prototypes to Manage Risk. Software Design and Publisher Magazine, 1996.

Stevens VJ, Funk KL, Brantley PJ, Erlinger TP, Myers VH, Champagne CM, Bauck A, Samuel-Hodge CD, Hollis JF. Design and Implementation of an Interactive Website to Support Long-Term Maintenance of Weight Loss.J Med Internet Res 2008;10:e1.

Streiner D, Norman, G. Health measurement scales. Oxford, Oxford University Press, 2003.

Tang ST, Huang YF, Hsiao ML, Yang SH, Young ST. Rapid prototyping strategy for a surgical data warehouse. Methods Inf Med 2003;42:243-50.

Tarzi S, Kennedy P, Stonec S, Evans M. Methicillin-resistant Staphylococcus aureus: psychological impact of hospitalization and isolation in an older adult population. J Hosp Infect 2001;49:250-4.

Teevan J, Alvarado C, Ackerman MS, Karger DR. The Perfect Search Engine Is Not Enough: A Study of Orienteering Behavior in Directed Search. In: Proceedings of the SIGCHI conference on Human factors in computing systems. New York, ACM Press 2004, 415-22.

The AGREE Collaboration. Development and validation of an international appraisal instrument for assessing the quality of clinical practice guidelines: the AGREE project. Qual Saf Health Care 2003;12:18-23. 
The Committee for Hospital Hygiene and Infection Prevention at the Robert Koch Institute. Empfehlung zur Prävention und Kontrolle von Methicillin-resistenten Staphylococcus Aureus (MRSA) in Krankenhäusern und anderen medizinischen Einrichtungen. (Recommendation for Prevention and control of Methicillin-resistant Staphylococcus Aureus (MRSA) in hospitals. Bundesgesundheitsbl Gesundheitsforsch Gesundheitsschutz 1999;42:954-8.

The Committee for Hospital Hygiene and Infection Prevention at the Robert Koch Institute. Infektionsprävention in Heimen. (Infection prevention in Nursing Homes). Bundesgesundheitsbl Gesundheitsforsch Gesundheitsschutz 2005;48:1061-80.

The Institute of Public Health. Hygienemaßnahmen zur Verhütung der Weiterverbreitung von MRSA in Krankenhäusern. (Hygiene Measures for the prevention of MRSA transmission in hospitals). Münster, Landesinstitut für den Öffentlichen Gesundheitsdienst, 2003.

The Institute of Public Health. Infektionsprävention in Heimen; Methicillin-resistenteStaphylococcus Aureus (MRSA). (Infection prevention in nursing homes; Methicillinresistante Staphylococcus aureus (MRSA). Münster, Landesinstitut für den Öffentlichen Gesundheitsdienst, 2005.

Thomas C, Bevan N. (eds). Usability Context Analysis: A Practical Guide. Teddington, National Physical

Thomas KW, Dayton CS, Peterson MW. Evaluation of internet-based clinical decision support systems. J Med Internet Res 1999;1:e6.

Thursky KA, Mahemoff M. User-centered design techniques for a computerised antibiotic decision support system in an intensive care unit. Int J Med Inform 2007;76:760-8.

Tigelaar A, Akker R op den, Verhoeven F. Matching Queries to Frequently Asked Questions: Search Functionality for the MRSA Web-Portal. In: Proceedings of the ninth Dutch-Belgian Information Retrieval Workshop, Enschede, 2009.

Tullis TS. Using Card-sorting Techniques to Organize your Intranet. J Intranet Strat Man 2003;1:1-9.

U.S. Department of Health and Human Services' Office of the Assistant Secretary for Public Affairs. Write scenarios. U.S. Department of Health and Human Services, Washington DC, 2009. URL: http://www.usability.gov/analyze/scenarios.html. [accessed: 2009 Apr 14]

Van Velsen L, Van der Geest T, Klaassen R, Steehouder M. User-centered evaluation of adaptive and adaptable systems: a literature review. Knowl Eng Rev 2008;23:261-81.

Vandenbroucke JP, Psaty BM. Benefits and risks of drug treatments: how to combine the best evidence on benefits with the best data about adverse effects. JAMA 2008;300:2417-9. 
Verhoeven F, Gemert-Pijnen JEWC van, Friedrich AW, Daniels-Haardt I, Hendrix MGR. Euregionale MRSA-preventie en bestrijding: een vergelijking van Duitse en Nederlandse richtlijnen. [Euregional prevention and control of MRSA: a comparison of German and Dutch guidelines]. Infectieziekten Bulletin 2007;04:125-9.

Verhoeven F, Gemert-Pijnen JEWC van, Friedrich AW, Daniels-Haardt I, Hendrix MGR, Steehouder MF. The development of a web-based information tool for cross-border prevention and control of Methicillin Resistant Staphylococcus aureus. Int J Infect Control 2008;4:1-11.

Verhoeven F, Vreeburg AJ, Friedrich AW, Daniels-Haardt I, Hendrix MGR, Steehouder MF, Gemert-Pijnen JEWC van. (a). Evaluatie van een web-based tool ter preventie en bestrijding van methicilline resistente Staphylococcus Aureus. Infectieziekten Bulletin 2009;20:26-8.

Verhoeven F, Vonderhorst S, Hendrix R, Gemert-Pijnen L van. (b). MRSA-dragers in het ongewisse. Medisch Contact 2009;4:150-54.

Verhoeven F, Steehouder MF, Hendrix MGR, Gemert-Pijnen, JEWC van. (c). User-centered development of a web-based tool to facilitate health care workers with infection control guidelines. In: Proceedings of the Etmaal of the Communicatiewetenschap, Nijmegen, 2009.

Verhoeven F, Steehouder MF, Hendrix MGR, Gemert-Pijnen JEWC van. (d). From expertdriven to user-oriented communication of infection control guidelines. Int J Hum Comput Int 2009. doi:10.1016/j.jjhcs.2009.07.2003

Verhoeven F, Steehouder MF, Hendrix MGR, Gemert-Pijnen JEWC van. (e). How nurses seek and evaluate clinical practice guidelines on the Internet. Accepted for publication in Journal of Advanced Nursing.

Verhoeven F, Steehouder MF, Hendrix MGR, Gemert-Pijnen JEWC van. (f). Factors affecting health care workers' adoption of a website with infection control guidelines. Int J Med Inform, 2009.

Voss A, Loeffen F, Bakker J, Klaassen C, Wulf M. Methicillin-resistant Staphylococcus aureus in Pig Farming. Emerg Infect Dis 2005;11:1965-6.

Vries EN de, Ramrattan MA, Smorenburg SM, Gouma DJ, Boermeester MA.The incidence and nature of in-hospital adverse events: a systematic review. Qual Saf Health Care 2008;17:216-23.

Weinman L. Designing Web Graphics. Indianapolis, New Riders, 1997.

Weitzman EA. Analyzing qualitative data with computer software. Health Serv Res 1999;34:1241-63.

Williams I, Dickinson H. Knowledge for adoption: a review of the literature on knowledge-based facilitators of technology adoption in health care. Birmingham, 
Health Services Management Centre/ NHS Institute for Innovation and Improvement, 2008.

Woolf S, Grol R, Hutchinson A, Eccles M, Grimshaw J. The potential benefits, limitations and harms of clinical guidelines. In: Eccles M, Grimshaw J, Eds. Clinical Guidelines. From Conception to Use. Radcliffe Medical Press Ltd., Oxon, 2000.

World Alliance for Patient Safety. Global patient safety challenge 2005-2006. Clean care is safer care. Geneva, WHO Document Production Services, 2005.

Wozar JA \& Worona PC. The use of online information resources by nurses. J Med Libr Assoc 2003:91:216-21.

Yu H, Lee M, Kaufman D, Ely J, Osheroff JA, Hripscsak G, Cimino J. Development, implementation, and a cognitive evaluation of a definitional question answering system for physicians. J Biomed Inform 2007;40:236-51.

Zhang J. Human-centered computing in health information systems Part 1: Analysis and design. J Biomed Inform 2005;38:1-3.

ZegelGezond. Een validatiemethode voor online publieksinformatie over gezondheid en ziekte. (A validation method for online public health information). Amsterdam,

Mediavedi, 2009. URL: http://www.zegelgezond.nl/index.php?pagina= 1 [accessed: 2009 Apr 23] 


\section{Samenvatting}

(Summary in Dutch) 



\section{Samenvatting (Summary in Dutch)}

\section{Introductie}

Om te voorkomen dat 'gevaarlijke' bacteriën zich verder verspreiden in en om het ziekenhuis, dient personeel zich te houden aan infectiepreventierichtlijnen. Hoewel ziekenhuispersoneel zich wel bewust lijkt te zijn van het belang van de richtlijnen, blijkt de naleving ervan in de praktijk niet optimaal. Dit wordt enerzijds veroorzaakt door contextuele factoren zoals een negatieve houding van het management ten aanzien van de richtlijnen of inadequate training van ziekenhuispersoneel over het uitvoeren van de richtlijnen. Anderzijds speelt het gebrek aan gebruiksvriendelijke documenten waarin de richtlijnen beschreven worden - protocollen genaamd - een rol bij onvoldoende naleving.

Uit eerder onderzoek weten we dat er vier redenen zijn die ervoor zorgen dat de gebruiksvriendelijkheid van protocollen niet optimaal is:

1) Protocollen moeten enerzijds wet- en regelgeving weergeven, maar anderzijds dient ziekenhuispersoneel ook in de protocollen te kunnen vinden welke maatregelen zij in de praktijk worden geacht te nemen. Door het combineren van deze twee soorten informatie ontstaan dikke en onleesbare documenten;

2) De protocollen worden opgesteld door experts op het gebied van infectiepreventie wat resulteert in veel jargon dat moeilijk te begrijpen is voor ziekenhuispersoneel;

3) Infectiepreventie-experts betrekken ziekenhuispersoneel niet in het ontwerpproces van de protocollen waardoor ziekenhuispersoneel geen binding krijgt met de protocollen;

4) De protocollen zijn vaak fysiek onvindbaar.

Een manier waarop de protocollen mogelijk gebruiksvriendelijker kunnen worden, is wanneer de richtlijnen via een website gecommuniceerd worden. Daarnaast moet ziekenhuispersoneel betrokken worden in het ontwerpproces van deze website. Op een website kunnen wet- en regelgeving gelinkt worden aan praktijkrichtlijnen zonder dat een dik en onleesbaar document ontstaat en zijn de richtlijnen altijd en overal te raadplegen. Door het betrekken van ziekenhuispersoneel bij het ontwerp van de website kunnen inhoud, structuur en vormgeving van de website afgestemd worden op hun wensen. Hierdoor zal personeel de richtlijnen beter begrijpen en daardoor adequater toepassen in de praktijk.

Het doel van dit onderzoek is het ontwerpen, implementeren en evalueren van een website met infectiepreventierichtlijnen waarbij ziekenhuispersoneel betrokken is in 
elke fase van het ontwikkelproces. Richtlijnen ter preventie en bestrijding van Methicilline Resistente Staphylococcus aureus (MRSA) dienen hierbij als casus. Het "usercentered model for website design", ontwikkeld door Kinzie en collega's (2002) vormt het uitgangspunt voor het ontwerpproces van de website. Dit model geeft stap voor stap aan hoe een website met richtlijnen ontwikkeld moet worden. Elk hoofdstuk in dit proefschrift beschrijft éen of meerdere stappen uit het model. In Figuur 1 is het model afgebeeld en gerelateerd aan de verschillende hoofdstukken van dit proefschrift.

\section{Hoofdstuk 2}

Analyseren van problemen

en behoeften

Hoofdstuk 3 \& 4

Behoeften prioriteren

\section{Hoofdstuk 3 \& 4}

Identificeren van oplos-

singen en vaststellen doelen

Hoofdstuk 3 \& 4

Ontwerp en ontwikkeling

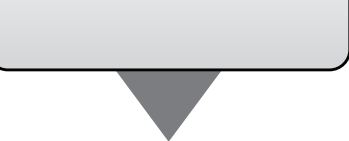

\section{Hoofdstuk 5}

Implementatie

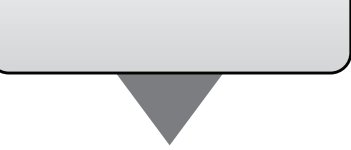

\section{Hoofdstuk 6}

Adoptie door gebruikers

Hoofdstuk 7

Gebruikers realiseren doelen

Figuur 1. Overzicht van de hoofdstukken in dit proefschrift 


\section{Hoofdstuk 2: Analyse van problemen en behoeften}

Infectiepreventierichtlijnen kunnen niet zomaar op een website geplaatst worden. Omdat het hier om documentatie van veiligheidsrichtlijnen gaat, moet aan een aantal kwaliteitscriteria worden voldaan. Deze criteria zijn opgesteld door de Internationale Organisatie voor Standaardisatie en houden het volgende in:

1) Status: Er moet duidelijk worden aangegeven wanneer de richtlijnen gemaakt zijn, tot wanneer ze geldig zijn en wie ze goedgekeurd heeft;

2) Evidence-based onderbouwing: Het nut van de richtlijnen moet door onderzoek aangetoond zijn;

3) Bereik: De doelgroep van de richtlijnen moet duidelijk worden gemaakt en direct worden aangesproken;

4) Logistiek: Er moet duidelijk worden aangegeven hoe de richtlijnen in de praktijk moeten worden uitgevoerd en door wie.

Aan de hand van een inhoudsanalyse van bestaande MRSA-richtlijnen werd vastgesteld hoe deze elementen in de praktijk verwerkt worden en hoe ze vertaald zouden kunnen worden naar een website. Hiertoe zijn de nationale MRSA-richtlijnen uit Duitsland, Engeland, Nederland en de Verenigde Staten geanalyseerd. Uit de analyse bleek dat er twee manieren zijn om bovenstaande criteria toe te passen. Enerzijds is er de expertbenadering (vooral aanwezig in de Duitse en Engelse richtlijnen) waarbij de nadruk ligt op status en evidence-based onderbouwing. Anderzijds is er de meer gebruikersgerichte benadering (vooral aanwezig in de Nederlandse en Amerikaanse richtlijnen), met veel aandacht voor bereik en logistiek, wat betekent dat de richtlijnen gecommuniceerd worden via beslisbomen, tabellen en figuren. Wanneer richtlijnen via een website gecommuniceerd worden, moeten echter beide benaderingen gecombineerd worden om aan de genoemde kwaliteitscriteria te voldoen. De inhoudsanalyse resulteerde in een checklist die aangeeft hoe kwaliteitscriteria voor richtlijnen toegepast moeten worden op een website. Deze checklist is gebruikt voor de inrichting van de website met MRSA-richtlijnen, die in het kader van dit promotieonderzoek werd ontwikkeld.

\section{Hoofdstuk 3: Behoeften prioriteren, identificeren van oplossing en ontwerp van de website}

Het toepassen van de checklist uit het vorige hoofdstuk is niet voldoende voor gebruikersgerichte communicatie van infectiepreventierichtlijnen. Ziekenhuispersoneel 
moet de website uiteindelijk in de praktijk gaan gebruiken, en daarom is aan hen gevraagd hoe de website vorm en inhoud moest krijgen. Hiertoe werd een viertal methoden van gebruikersonderzoek toegepast, naast de inhoudsanalyse uit hoofdstuk 2. De resultaten van de analyse uit hoofdstuk 2 worden in dit hoofdstuk kort herhaald.

Vervolgens werden 28 praktijktesten uitgevoerd met ziekenhuispersoneel. Ziekenhuispersoneel voerde hard-op-denkend taken uit aan de hand van het papieren MRSA-protocol. De resultaten van deze praktijktesten werden gebruikt om twee vragen te beantwoorden. Ten eerste werden de wensen en behoeften van ziekenhuispersoneel ten aanzien van de website vastgesteld. Deze wensen en behoeften worden in dit hoofdstuk beschreven. Ten tweede werd op basis van de resultaten vastgesteld welke problemen zich voordoen met het gebruik van de bestaande, papieren MRSA-richtlijnen. Deze problemen worden in hoofdstuk 4 beschreven. Ook werd een vragenlijst afgenomen om vast te stellen welke factoren een rol spelen bij de naleving van de richtlijnen die op de website gecommuniceerd worden. In dit hoofdstuk worden de resultaten slechts samengevat; hoofdstuk 7 gaat dieper in op de resultaten.

Uit de praktijktesten bleek dat ziekenhuispersoneel graag een website wil waarop richtlijnen via een vraag-antwoord-structuur gecommuniceerd worden. In het tweede gebruikersonderzoek werden daarom 10 ziekenhuismedewerkers verzocht om 165 vragen over de MRSA-richtlijnen in te delen in voor hen logische categorieën en deze categorieën vervolgens te benoemen. Via speciale software (WebSort ${ }^{\mathrm{TM}}$ ) werd de optimale structuur van de vragen berekend, resulterend in een menustructuur voor de website.

Het derde gebruikersonderzoek was bedoeld om de vormgeving van de website vast te stellen. Twee prototypes van de website werden voorgelegd aan 14 respondenten en op basis van hun opmerkingen werd de uiteindelijke vormgeving van de website gekozen.

\section{Hoofdstuk 4: Behoeften prioriteren, identificeren van oplossing en ontwerp van de website}

In dit hoofdstuk wordt verslag gedaan van twee onderzoeken. In het eerste onderzoek, dat reeds kort aan bod kwam in hoofdstuk 3, werd achterhaald welke problemen zich voordoen wanneer ziekenhuismedewerkers papieren MRSA-protocollen gebruiken. Op basis van de uitkomsten werd de eerste werkende versie van de website ontwikkeld. In het tweede onderzoek werd bepaald in hoeverre de problemen die optraden bij het gebruik van de papieren protocollen, door de website verholpen werden.

\section{Onderzoek 1}

Gemiddeld besteedden respondenten 383 seconden ( 6.5 minuut) aan een taak wanneer zij het papieren protocol gebruiken. Ruim $52 \%$ van de taken kon niet succesvol opgelost worden vanwege in totaal 270 problemen op drie niveaus: 
1) Onduidelijke informatiestructuur (41 problemen, zoals het ontbreken van een index);

2) Mismatch tussen het vocabulaire van de ziekenhuismedewerker en de richtlijnauteurs ( 76 problemen, bijvoorbeeld: auteurs gebruiken het woord 'decontaminatieprocedure', terwijl ziekenhuispersoneel dit 'behandeling' noemt);

3) Onvoldoende kwaliteit van de informatie (onbegrijpelijk, inaccuraat en onvolledig; 153 problemen, zo stond er bijvoorbeeld achterhaalde informatie uit 2002 in de richtlijnen).

Het onderzoek leverde drie ontwerpprincipes op voor de website:

1) Actiegeoriënteerde communicatie van richtlijnen via een vraag-antwoordstructuur, in de taal van ziekenhuispersoneel;

2) Multimediale presentatie van richtlijnen (zoveel mogelijk gevisualiseerd via video, afbeeldingen, bronnen etc.);

3) Optimale navigatie (stelt gebruiker in staat via eigen zoekstrategie te zoeken via categorieën, zoekmachine of meest gestelde vragen).

Op basis van deze ontwerpprincipes werd de eerste werkende versie van de website ontwikkeld.

\section{Onderzoek 2}

Om vast te stellen of de problemen die in onderzoek 1 aan het licht kwamen, werden verholpen door de website, werden dezelfde taken één jaar later door dezelfde 28 ziekenhuismedewerkers uitgevoerd als in onderzoek 1, maar dit keer aan de hand van de website. Bijna $88 \%$ van de taken werd succesvol uitgevoerd in gemiddeld 132 seconden (2.2 minuut). De website stelt ziekenhuispersoneel dus in staat om sneller en beter naar MRSA-richtlijnen te zoeken. Hoewel het aantal mismatchproblemen (van 76 naar 49) en informatiekwaliteitsproblemen (van 153 naar 124) afnam, nam het aantal structuurproblemen toe (van 41 naar 69). Dat kwam waarschijnlijk doordat ziekenhuispersoneel gewend is om richtlijnen vanaf papier te lezen en niet vanaf een website. Dit tweede onderzoek diende tevens als formatieve evaluatie van de website. Systeemfouten, onduidelijkheden en inconsistenties werden verholpen voordat de website gelanceerd werd.

\section{Hoofdstuk 5: Implementatie}

Op 19 februari 2008 ging de website met MRSA-richtlijnen online (zie www. mrsa-net.nl). Echter, op internet zijn nog veel meer websites beschikbaar over 
MRSA en het is daarom de vraag hoe ziekenhuispersoneel de website beoordeelt te midden van andere relevante websites. Daarom werden in dit onderzoek 20 ziekenhuismedewerkers gevraagd om hard-op-denkend taken uit te voeren via internet.

Ziekenhuispersoneel bleek www.mrsa-net.nl goed te waarderen in vergelijking met andere MRSA-websites, omdat ze de richtlijnen op www.mrsa-net.nl relevanter en completer vonden. Relevant betekende in dit geval dat de informatie aansluit op de dagelijkse praktijk. Compleet hield in dat de informatie iets toevoegt aan de bestaande richtlijnen en dan vooral met betrekking tot de persoonlijke risico's van MRSA voor ziekenhuispersoneel. Zaken die website-experts belangrijk vinden, zoals accuraatheid en betrouwbaarheid van de bron, vond ziekenhuispersoneel minder belangrijk. Zolang richtlijnen aansluiten op wat ziekenhuispersoneel al weet en daar praktische informatie aan toevoegt waar ze echt iets mee kan, is ziekenhuispersoneel tevreden.

Het ziekenhuispersoneel vond het niet prettig dat de website gemaakt is door instanties die zij niet kent. Daardoor is personeel er niet zeker van of ze de richtlijnen in de praktijk wel mogen gebruiken van hun afdelingshoofden. Daarom zou op www. mrsa-net.nl beter moeten worden aangegeven dat de richtlijnen op de website overeenkomen met de verplichte nationale MRSA-richtlijnen van de Werkgroep Infectie Preventie.

Verder bleek ziekenhuispersoneel het moeilijk te vinden om op internet te zoeken naar MRSA-richtlijnen, onder andere omdat zij de juiste zoektermen niet kon bedenken of omdat informatie niet stond op de plaats waar personeel dat verwachtte. Daarom is het belangrijk dat makers van richtlijnwebsites rekening houden met de zoekstrategieën van ziekenhuispersoneel en het hen zo gemakkelijk mogelijk maakt. Zo moet ervoor gezorgd worden dat de zoekmachine het taalgebruik van ziekenhuismedewerkers herkent en moet er vooral veel praktische informatie aan de richtlijnen toegevoegd worden, zoals het antwoord op de volgende vraag: "Ik ben verpleegkundige en heb zelf MRSA. Mag ik op visite bij mijn zieke moeder?"

\section{Hoofdstuk 6: Adoptie door gebruikers}

De vorige hoofdstukken lieten zien dat de website ziekenhuispersoneel in staat stelt om sneller te zoeken naar MRSA-richtlijnen en met een beter resultaat. Dat de website gebruiksvriendelijk wordt bevonden door de doelgroep, betekent echter niet dat ziekenhuispersoneel de website in de praktijk ook daadwerkelijk gebruikt. Via 20 interviews met ziekenhuispersoneel werd daarom in kaart gebracht welke factoren bepalen dat ziekenhuispersoneel de website gaat gebruiken in haar dagelijkse werkomgeving. Het PRECEDE model, een veelgebruikt model in de gezondheidsvoorlichting, vormde hierbij het analysekader.

Uit de interviews bleek dat er verschillende soorten factoren van invloed zijn op het gebruik van de website in de praktijk: 
1) Organisatiefactoren: Ziekenhuishygiënisten moeten de website aanbevelen onder ziekenhuispersoneel omdat zij worden gezien als de belangrijkste opinieleiders op dit gebied. Verder moeten de makers van de website ziekenhuispersoneel persoonlijk benaderen en op het nut van de website wijzen omdat persoonlijke, informele communicatie werd verkozen boven andere mechanismen;

2) Werkgerelateerde factoren: De inhoud van de website moet inspelen op de verschillen in kennis tussen de verschillende doelgroepen. Zo hebben microbiologen een andere informatiebehoefte dan afdelingsassistenten. Verder zouden de ziekenhuishygiënisten moeten benadrukken dat het gebruik van de website de werkdruk verlaagt in plaats van verhoogt;

3) Individuele factoren: Sommige mensen gaven aan weerstand te hebben tegen de website omdat zij liever op basis van eigen kennis en ervaring handelen dan op een website te vertrouwen. Ziekenhuishygiënisten moeten daarom aangeven dat de richtlijnen op de website overeenkomen met de landelijke, verplichte MRSArichtlijnen en dat iedereen, ook personeel met weerstand, deze website daarom kan gebruiken;

4) Websitefactoren: Ziekenhuispersoneel bleek vooral het gemak (efficiëntie) waarmee naar praktische informatie gezocht kan worden op de website (effectiviteit) te waarderen. Ziekenhuispersoneel zag de website vooral als aanvulling op de bestaande richtlijnen. Dit moet door ziekenhuishygiënisten benadrukt worden wanneer zij de website promoten onder ziekenhuispersoneel.

Wanneer de websitemakers het gebruik van de website in de praktijk willen stimuleren, dienen zij in te spelen op bovenstaande factoren. Het is daarbij belangrijk te beginnen met de organisatiefactoren omdat blijkt dat ziekenhuispersoneel ondersteuning/ goedkeuring vanuit het ziekenhuis de belangrijkste reden vindt om de website te gaan gebruiken.

\section{Hoofdstuk 7: Gebruikers realiseren doelen}

Ook al wordt de gebruiksvriendelijkheid van de website positief beoordeeld door ziekenhuispersoneel, dit betekent nog niet dat ziekenhuispersoneel zich in de praktijk ook aan de richtlijnen zal houden die op de website staan. Dit hoofdstuk beschrijft de factoren die bepalen of ziekenhuispersoneel zich wel of niet aan de richtlijnen zal houden, gemeten via een vragenlijst $(n=217)$ en interviews $(n=24)$ afgenomen onder ziekenhuispersoneel, wederom gebaseerd op het PRECEDE model.

Het onderzoek liet drie soorten factoren zien die van invloed zijn op het naleven van richtlijnen (hieronder elk genoemd met een voorbeeld):

1) Organisatiefactoren: Het veiligheidsbewustzijn in het ziekenhuis bleek de 
belangrijkste factor voor het naleven van de richtlijnen. Als er in het ziekenhuis een sterk veiligheidsbewustzijn is, is ziekenhuispersoneel zich bewust van de risico's van het niet naleven van de richtlijnen en het belang van het wel naleven. Gezamenlijk probeert ziekenhuispersoneel er alles aan te doen om te voorkomen dat bacteriën zoals MRSA zich verder in het ziekenhuis verspreiden. In de literatuur wordt een sterk veiligheidsbewustzijn in een organisatie gekenmerkt door management dat duidelijk laat merken belang te hechten aan naleving van de richtlijnen, goede training op het gebied van richtlijnen en personeel dat elkaar onderling feedback geeft wanneer zij zien dat een ander zich niet aan de richtlijnen houdt. Hoewel uit de literatuur blijkt dat de opvattingen van het management de belangrijkste voorwaarde vormen voor veiligheidsbewustzijn, gaf ziekenhuispersoneel in dit onderzoek aan dat training en feedback belangrijker zijn;

2) Werkgerelateerde factoren: Een hoge werkdruk is een belangrijke reden waardoor ziekenhuispersoneel niet altijd in staat is zich aan de richtlijnen te houden;

3) Individuele factoren: Artsen bleken negatiever te zijn over het nut van de richtlijnen en waren minder bereid om zich aan te passen dan verpleegkundigen.

Bij een sterk veiligheidsbewustzijn kunnen de gevaren van het niet naleven van de richtlijnen openlijk worden besproken, wat er mogelijk toe leidt dat ondanks een hoge werkdruk en persoonlijke weerstand tegen de richtlijnen, iedereen zich uiteindelijk beter aan de richtlijnen houdt.

\section{Conclusie}

De methoden die in dit onderzoek gebruikt zijn voor de ontwikkeling van de website met MRSA-richtlijnen, geven inzicht in hoe aan kwaliteitscriteria voldaan kan worden als richtlijnen worden gecommuniceerd via een website (hoofdstuk 2), de wijze waarop richtlijnen gestructureerd en gepresenteerd moeten worden op een website (hoofdstuk $3,4,5$ ) en factoren die in acht genomen moeten worden bij de invoering van de website in de dagelijkse praktijk (hoofdstuk 6 en 7).

Deze methoden van gebruikersonderzoek hebben geleid tot een website die ziekenhuispersoneel in staat stelt om efficiënter (ruim 4 minuten) en effectiever ( $40 \%$ meer geslaagde zoekopdrachten) te zoeken naar MRSA-richtlijnen vergeleken met papieren protocollen. Ziekenhuispersoneel is meer tevreden over de manier waarop de richtlijnen worden gecommuniceerd op de website dan in papieren protocollen, omdat de richtlijnen op de website meer aansluiten bij de belevingswereld van ziekenhuispersoneel door de actiegeoriënteerde communicatie van richtlijnen, de multimodale presentatie en de optimale navigatiestructuur.

Het onderzoek bevestigt dat infectiepreventie-experts die de (papieren) protocollen maken, een heel andere kijk hebben op de manier waarop richtlijnen gestructureerd, gepresenteerd en ingevuld moeten worden dan degenen die de richtlijnen moeten 
uitvoeren. Alleen door ziekenhuispersoneel actief te betrekken bij het ontwerpproces van richtlijncommunicatie, kan een manier worden gevonden om richtlijnen zo te communiceren dat ziekenhuispersoneel er effectief en efficiënt mee om kan gaan.

Daarnaast blijkt dat organisatiefactoren zoals training en feedback veel invloed hebben op het gebruik van de website in de praktijk en het naleven van de richtlijnen die op de website staan. Het is dus niet genoeg om te zorgen dat een website met richtlijnen gebruiksvriendelijk is, maar er moet ook voor gezorgd worden dat de website past binnen de context waarin ziekenhuispersoneel werkt.

Behalve in het onderzoek dat wordt beschreven in hoofdstuk 5 , waren in elk onderzoek waarvan dit proefschrift verslag doet, ziekenhuismedewerkers uit zowel Nederland als Duitsland betrokken. Omdat de Duitse cultuur gekenmerkt wordt door een hoge mate van onzekerheidsvermijding en machtsafstand vergeleken met de Nederlandse cultuur, werd verwacht dat Duits ziekenhuispersoneel andere behoeften ten aanzien van richtlijncommunicatie zou hebben en meer geneigd zou zijn tot richtlijnnaleving dan Nederlands ziekenhuispersoneel. De verschillen bleven echter uit. Een mogelijke verklaring hiervoor is het feit dat het onderzoek vooral werd uitgevoerd in een grensgebied, waar een soort euregionale mentaliteit bestaat en individuele normen en waarden afwijken van de nationale cultuur. Cultuurverschillen bleken echter wel te bestaan tussen beroepsgroepen: artsen en verpleegkundigen verschilden van mening zowel ten aanzien van de website als van richtlijnnaleving. In interventies dient rekening gehouden te worden met deze verschillen.

In toekomstig onderzoek moet de toepasbaarheid van de gebruikersgerichte methode getest worden in andere contexten. Ook moet onderzocht worden hoe de functies van de website uitgebreid kunnen worden om de website nog beter te laten aansluiten op de dagelijkse praktijk van ziekenhuispersoneel. 


\section{Dankwoord}

Acknowledgements in Dutch 



\section{Dankwoord (Acknowledgements in Dutch)}

MRSA betekent Methicilline Resistente Staphylococcus aureus. Ik had er nog nooit van gehoord totdat ik vier jaar geleden aan mijn promotieonderzoek begon. Inmiddels weet ik dat MRSA niet alleen gevaarlijke bacteriën zijn, maar weet ik ook dat MRSA staat voor Met Structuur en Regelmaat Arbeid verrichten; Moe, Radeloos, Stressvol en Afgepeigerd, maar ook Met Ron Samen naar Alex of Mooie Resultaten Samenvoegen in een Artikel.

De mensen met wie ik de afgelopen vier jaar heb samengewerkt aan dit project hebben mij dit brede perspectief "geschonken". Dat is het mooiste dat het onderzoek mij persoonlijk heeft opgeleverd. Veel mooier nog dan dit proefschrift of de website die erbij hoort. Een aantal mensen verdient het om hier genoemd te worden.

Lisette: dagelijks begeleidster. Wat een bijzonder mens ben je! Ik had me geen betere begeleidster kunnen wensen. Altijd, zelfs in het weekend of "s nachts, reageerde je à la minute op mijn vragen. Je feedback was constructief, ook al kon ik je handschrift niet altijd lezen. Ik heb veel van je geleerd en niet alleen met betrekking tot werk. Gelukkig zijn we voorlopig nog niet van elkaar af!

Michaël: promotor, maar vooral ook mede-auteur. Wat een betrokkenheid toonde je bij de totstandkoming van mijn publicaties en wat waren je aanvullingen of herformuleringen sterk! Daarnaast waardeer ik de persoonlijke manier waarop je met je promovendi omgaat. Dat houdt ons op de been.

Ron: de microbioloog. Zo noem ik je altijd als ik tegen anderen, die jou niet kennen, over je spreek. Ten eerste bedankt voor je medische expertise. Zonder jouw hulp had de website geen inhoud gehad, maar alleen vorm. Ten tweede bedankt voor je commentaren op mijn artikelen; het was verfrissend om eens niet met een communicatiewetenschapper van gedachten te wisselen. Ten derde bedankt voor alle wijze lessen in de auto van en naar Münster.

Alexander F und Inka: Ich fand es großartig mit euch zusammen zu arbeiten. Euer Enthusiasmus, Engagement und Fachwissen wirkten sehr ansteckend. Ich freue mich schon um weiterhin mit euch am EURSAFETY Projekt zu arbeiten! Alexander $M$ und Robin, ein Dankeschön für euer fachinhaltliches Wissen.

I am very thankful to the members of my graduation committee for taking the time and effort to read my thesis and admitting it to the public defence.

De vakgroep Technische en Professionele Communicatie: mijn collega's. In het bijzonder bedank ik Arjenne, Lex, Sanne S, Nicole, Nalini, Carolina, Joyce, Thea en Marieke $\mathrm{W}$ voor jullie advies en inspiratie. 
Almer, Harm en Wilko: het brein achter de website. Onze samenwerking is zeer vruchtbaar en prettig verlopen. Door jullie technische inzicht is er een website gecreëerd die dagelijks inmiddels honderden bezoekers aantrekt. Veel dank!

Mark: vormgever. Niet alleen de website, maar ook het proefschrift heb je weten om te toveren tot een lust voor het oog (vind ik). Dank daarvoor.

Frank, Franca en Juliette: fotograaf en modellen. Dank voor jullie medewerking aan de kaft van dit proefschrift. Die is precies goed zo. Franca, het is fijn om een vriendin letterlijk op het proefschrift te hebben staan.

De hygiënisten van het MST, SKB, UKM en $\mathrm{CH}$ : zonder jullie medewerking had ik niet in korte tijd aan zoveel respondenten voor het onderzoek kunnen komen. Ik heb ervaren hoeveel hart jullie hebben voor infectiepreventie. Daar moeten jullie toch menig ziekenhuismedewerker mee kunnen "infecteren”: weg met die MRSA!

Alle respondenten die op welke manier dan ook hebben meegewerkt aan mijn onderzoek: user-centered design staat of valt bij een betrokken doelgroep. Jullie vormden met zijn allen het ultieme voorbeeld van hoe een gebruikersgeoriënteerd ontwerpproces zou moeten gaan. Merci!

Ansfrida, Elena en Jennifer: student-assistenten. Fijn dat jullie al het rotwerk voor me hebben opgeknapt! Gelukkig zijn we nu nog steeds bevriend. Sanne ten T, jij ook bedankt voor al je typewerk, maar nog veel meer bedankt voor de leuke anderhalf jaar die we samen hebben doorgebracht aan de Rembrandtlaan.

Susanne, Elise, Judith, Anneke en Emilie: afstudeerders binnen het MRSA-project. Allemaal hebben jullie een onmisbare bijdrage geleverd aan het publieke deel van de website. Dank daarvoor! Echter, nog veel meer bedankt voor de vriendschap die ik met sommige van jullie nog steeds heb.

De JAAARclub: Karin, Nina, Nelly, Jojanneke, Saskia, Renske, Nicol, Nicole, Marieke W, Marieke $\mathrm{H}$ en Loes. Wat een feest was het om met jullie de sleur van het promoveren te ontvluchten en nachtenlang te stappen, te high-tea'en, en vooral heel, heel veel te kletsen en dan met name niet over onderzoek.

Nicol: Kamergenoot (bijna) vanaf het begin en vriendin. Wat een energie en wat een spontaniteit straal jij uit, zeg! Zelfs toen ik drie weken met je op vakantie was, kreeg ik geen genoeg van je grappen en grollen. Que bonita es esta vida!

Nienke: dierbare vriendin. We zijn al in elkaars leven sinds het begin van onze studie in 1999. De band werd steeds hechter en bereikte een toppunt toen je bij me introk, in 2005 . Wat een geweldige momenten hebben we toen beleefd! Ik ben heel blij dat jij mijn paranimf wilt zijn.

Renske: mijn hartsvriendin. De afgelopen vier jaar hebben we alles samen gedeeld, beschouwd, aanschouwd, gekocht, gemaakt, bereisd, gegeten, gedronken, gezocht, gevonden, maar vooral besproken. Bedankt voor wie je bent en je onvoorwaardelijke vriendschap. Natuurlijk ben jij ook mijn paranimf. LL321. 
Papa en mama. Precies op de momenten waarop ik het nodig had sprongen jullie "even" in de auto naar Enschede. Altijd was ik welkom in Best om te typen aan het proefschrift of om gewoon even uit te razen. Het had niet beter gekund. Alles kan bij jullie, maar niets hoeft, want trots zijn jullie toch wel. En dat is het beste gevoel dat een dochter zich kan wensen.

Jesse. Bedankt voor alles. Voor je afleiding op de juiste momenten, voor je relativeringsvermogen, voor je luisterende oor, voor je geduld. En niet te vergeten de culinaire hoogstandjes die altijd weer klaar staan als ik (laat) thuiskom. Je bent er van het begin af aan bij geweest en je had geen moment later moeten komen. Door en met jou heb ik een thuis in de mooiste stad van Nederland.

Fenne Verhoeven

Utrecht, september 2009 
List of publications 



\section{List of publications}

Verhoeven F, Friedrich AW, Gemert-Pijnen JEWC, Mellmann A, Hendrix MGR, DanielsHaardt I. EUREGIO MRSA-net Twente-Muensterland - Network development for the combat of MRSA under involvement of the public health service. Gesundheitswesen 2006;3:193.

Friedrich AW, Daniels-Haardt I, Sonntag AK, Verhoeven F, Gemert-Pijnen L, Mellman A, Hendrix R. EUREGIO MRSA-net Twente/Münsterland. Westfälisches Ärzteblatt 2006;9:5 1-2.

Daniels-Haardt I, Verhoeven F, Mellmann A, Hendrix MGR, Gemert-Pijnen JEWC van, Friedrich AW. EUREGIO-Projekt MRSA-net Twente/Münsterland: Regionale Netzwerkbildung zur Bekämpfung von MRSA. Gesundheitswesen 2006;68:674-8.

Verhoeven F, Gemert-Pijnen JEWC van, Friedrich AW, Daniels-Haardt I, Hendrix MGR. Euregionale MRSA-preventie en bestrijding: een vergelijking van Duitse en Nederlandse richtlijnen. Infectieziekten Bulletin 2007;04:125-9. [Base for Chapter 2 of this thesis]

Verhoeven F, Gemert-Pijnen JEWC van, Friedrich AW, Daniels-Haardt I, Hendrix MGR, Steehouder MF. A web-based infectious diseases communication system to enhance health care workers' knowledge, attitude, and risk perception about infection control guidelines. Technology and Health Care 2007:5;366-7.

Friedrich AW, Daniels-Haardt I, Gemert-Pijnen JEWC van, Hendrix MGR, Eiff C von, Kipp F, Becker K, Köck R, Verhoeven F, Mellman A. Ein regionales Netzwerk zur Prävention und Kontrolle von Infektionen durch MRSA: EUREGIO MRSA-net Twente/Münsterland. Epidem Bull 2007,33;307-11.

Friedrich AW, Daniels-Haardt I, Köck R, Verhoeven F, Mellmann A, Harmsen D, GemertPijnen JEWC van, Becker K, Hendrix MGR. EUREGIO MRSA-net Twente/Münsterland- A Dutch-German cross-border network for the prevention and control of infections caused by Methicillin-Resistant Staphylococcus aureus. Euro Surveill 2008;35.

Verhoeven F, Gemert-Pijnen JEWC van, Friedrich AW, Daniels-Haardt I, Hendrix MGR, Steehouder MF. The development of a web-based information tool for cross-border prevention and control of Methicillin Resistant Staphylococcus aureus. Int J Infect Control 2008;4. [Base for Chapter 3 of this thesis]

Verhoeven F, Gemert-Pijnen JEWC van, Friedrich AW, Daniels-Haardt I, Hendrix MGR. Ontwikkeling van een web-based learning tool ter preventie en bestrijding van Meticilline Resistente Staphylococcus aureus. Infectieziekten Bulletin 2008;1:6-9.

Verhoeven F, Gemert-Pijnen JEWC van, Friedrich AW, Daniels-Haardt I, Hendrix MGR. Ontwikkeling van een web-based learning tool ter preventie en bestrijding van Methicilline resistente Staphylococcus aureus. Microlabblad 2008;1:329-32. 
Verhoeven F, Gemert-Pijnen JEWC van, Friedrich AW, Daniels-Haardt I, Hendrix MGR. Entwicklung eines Web-basierten learning tools zur Prävention und Bekämpfung von Methicillin Resistenten Staphylococcus aureus (MRSA). Hyg Med 2008;33:412-6.

Verhoeven F, Gemert-Pijnen JEWC van, Friedrich AW, Daniels-Haardt I, Hendrix MGR. De eerste objectieve resultaten van een web-based learning tool rondom MRSA: www. mrsa-net.nl. Microlabblad 2008;2:35 1-3.

Verhoeven F, Karreman J, Bosma AR, Hendrix MGR, Gemert-Pijnen JEWC van. Voorlichting over Methicilline Resistente Staphylococcus aureus: Een onderzoek naar de op- en misvattingen van het Nederlandse publiek. Tijdschrift voor Hygiëne en InfectiePreventie 2008;5:119-24.

Tigelaar A, Akker R op den, Verhoeven F. Matching Queries to Frequently Asked Questions: Search Functionality for the MRSA Web-Portal. In: Proceedings of the ninth Dutch-Belgian Information Retrieval Workshop, Enschede, 2009.

Verhoeven F, Vreeburg AJ, Friedrich AW, Daniels-Haardt I, Hendrix MGR, Steehouder MF, Gemert-Pijnen JEWC van. Evaluatie van een website ter preventie en bestrijding van Methicilline Resistente Staphylococcus aureus. Infectieziekten Bulletin 2009;20:26-8.

Verhoeven F, Vonderhorst S, Hendrix R, Gemert-Pijnen L van. MRSA-dragers in het ongewisse. Medisch Contact 2009;4:150-54.

Verhoeven F, Steehouder MF, Hendrix MGR, Gemert-Pijnen JEWC van. From expertdriven to user-oriented communication of infection control guidelines. Int J HumComput Stud 2009. doi:10.1016/j.ijhcs.2009.07.2003 [Base for Chapter 4 of this thesis]

Verhoeven F, Steehouder MF, Hendrix MGR, Gemert-Pijnen JEWC van. How nurses seek and evaluate clinical practice guidelines on the Internet. Accepted for publication in J Adv Nurs. [Base for Chapter 5 of this thesis]

Verhoeven F, Steehouder MF, Hendrix MGR, Gemert-Pijnen JEWC van. Factors affecting health care workers' adoption of a website with infection control guidelines. Int J Med Inform 2009. doi: 10.1016/j.ijmedinf.2009.06.001 [Base for Chapter 6 of this thesis]

Verhoeven F, Karreman J, Bosma AR, Hendrix MGR, Gemert-Pijnen JEWC van. Toward improved education of the public about methicillin-resistant Staphylococcus aureus: A Mental Models Approach. Accepted for publication in Int J Infect Control. 
224 